\title{
Endbericht
}

\section{Evaluierung des AplusB-Programms}

Michael Ploder, Jürgen Streicher, Enikö Linshalm (JOANNEUM RESEARCH)

in Kooperation mit

Karin Grasenick, Renate Handler (convelop gmbH)

Jürgen Egeln (Zentrum für Europäische Wirtschaftsforschung - ZEW)

Im Auftrag des Bundesministeriums für Verkehr, Innovation und Technologie

Wien/Graz, im November 2015

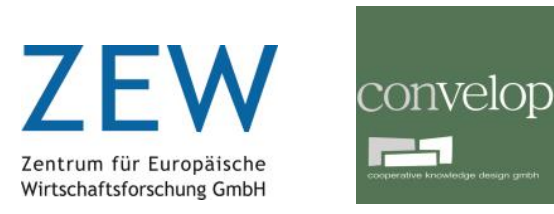

JOANNEUM

RESEARCH

POLICIES 


\section{Endbericht der Evaluierung des AplusB-Programms}

Michael Ploder, Jürgen Streicher, Enikö Linshalm (JOANNEUM RESEARCH)

Karin Grasenick, Renate Handler (convelop GmbH)

Jürgen Egeln (Zentrum für Europäische Wirtschaftsforschung - ZEW)

JOANNEUM RESEARCH Forschungsgesellschaft mbH

Institut für Wirtschafts- und Innovationsforschung

Büro Graz

Leonhardstraße 59

A-8010 Graz, Austria

Tel.: +43-316-876 1488

E-Mail: policies@joanneum.at
Büro Wien

Haus der Forschung, Sensengasse 1

A-1090 Wien, Austria

Tel.: +43-1-5817520

E-Mail: policies@joanneum.at 


\section{Auf einen Blick}

Akademische Spin-off-Gründungen sind heute wesentlicher Bestandteil eines effektiven Wissens- und Technologietransfers zwischen Wissenschaft und Wirtschaft. Eine zentrale Kraft in der österreichischen Förderungslandschaft im Bereich der innovativen Gründungen aus dem akademischen Umfeld stellt seit 2001 das Academia plus Business (AplusB) Programm dar. Im Zuge der vorliegenden Evaluierung wurde das AplusB-Programm auf Basis eines Multi-Ebenen-Ansatzes sowie unter Einbindung regionaler Stakeholder einer gesamtheitliche Betrachtung der erzielten Wirkungen unterzogen. Die Bewertung basiert auf Literatur- und Dokumentenauswertungen, einer Logic-Chart-Analyse, leitfadengestützten Interviews, einer Sekundärdatenanalyse mit Kontrollgruppenvergleich, Fallstudien sowie Fokusgruppen.

Das AplusB-Programm fokussiert auf innovative, in der Regel technologieorientierte, komplexe, in der Betreuung über einen längeren Zeitraum aufwendige Gründungsvorhaben, die in Hinblick auf strukturellen Wandel und Wachstum volkswirtschaftlich von Bedeutung sind. Weder wissenschaftlichen Einrichtungen noch private Inkubatoren können dieses von der Frühphase der Gründungen an mit hohem entwicklungs- und Betreuungsaufwand verbundene Segment hinreichend bedienen. Das Leistungsangebot der AplusB-Zentren ist auf die regionalen Anforderungen und Strukturen (Wissenschaft, Wirtschaft, Fördereinrichtungen und die Start-up-Szene) abgestimmt.

Das AplusB-Programm hat seit seinem Bestehen wesentlich zur Bewusstseinsbildung bei AkademikerInnen und wissenschaftlichen Einrichtungen sowie zur Anregung von Gründungen aus dem akademischen Umfeld beigetragen. Der Bekanntheitsgrad hat sich durch die Beteiligung an Wettbewerben stark erhöht, auch international gewann das Programm an Aufmerksamkeit. Das Programm AplusB und die Arbeit der AplusB-Zentren werden von den betreuten Gründungen sehr positiv bewertet. Die AplusB-Zentren werden als vertrauenswürdiger Partner mit Kompetenz in mehreren Themenfeldern, hoher Professionalität, Glaubwürdigkeit, Motivation und einem starken und wachsenden Netzwerk wahrgenommen, der für Unabhängigkeit ohne Eigeninteresse steht.

Von 2002 bis Anfang 2014 wurden 486 Unternehmen mit Unterstützung der Zentren gegründet, davon waren 1t. Gründungs-Monitoring lediglich rd. $9 \%$ Gründerinnen. Rd. $30 \%$ der Vorhaben wären ohne Unterstützung von AplusB nicht umgesetzt worden, weitere $52 \%$ lediglich in reduzierter Form. In Abgleich mit der offiziellen Statistik zur Unternehmensdemographie zeigt sich, dass bis Anfang 2014 noch 386 dieser Unternehmen wirtschaftlich aktiv waren. Durch die gegründeten Unternehmen wurden zw. 2002 und 2015 lt. Eigenerhebung der AplusB-Zentren rd. 3.400 Arbeitsplätze geschaffen. Lt. offizieller Beschäftigungsstatistik waren Ende 2013 in unabhängigen aktiven AplusB-Unternehmen 1.752 Personen in Österreich beschäftigt. Im Vergleich zu einer Kontrollgruppe weisen AplusB-Gründungen eine höhere Überlebensrate und Beschäftigungswirkung, dynamischere Umsatzentwicklung, höhere Exportrate sowie F\&E-Intensität auf. Auf regionaler Ebene konnten keine wesentlichen Abweichungen bei den Ergebnissen festgestellt werden.

Vor dem Hintergrund des gezeichneten Bildes erscheint die Weiterführung des Programms aus heutiger Perspektive als sinnvoll und notwendig. Für die künftige Entwicklung ist Augenmerk auf eine weitergehende Abstimmung und Konzeption auf nationaler wie regionaler Ebene zu legen, auf eine stärkere Profilbildung sowie eine Definition von Nahtstellen zu privaten Anbietern, auf das Kernstück „High-End“-Gründungen, eine klarere Festlegung von Wirkgrößen in Verbindung mit einem entsprechenden Monitoring, eine Anpassung bzw. Präzisierung von Programmzielen im Hinblick auf mögliche neue Entwicklungsbereiche und eine Verbesserung bei gendergerechten Angeboten, um auch einen verbesserten, direkten, Zentren-übergreifenden Austausch von GründerInnen auf der nationalen Ebene zu ermöglichen. 


\section{In a Nutshell}

Today, academic spin-offs are essential in facilitating effective knowledge and technology transfer across the fields of science and business. Since 2001, Academia plus Business (the AplusBProgramme) has played a central role in the Austrian funding landscape in the support of innovative, academic start-ups. The present evaluation draws upon a holistic assessment perspective by adopting a multi-level approach and by including the programme impact on regional stakeholders. The assessment is based on an evaluation of the relevant literature and documentation, logic chart analysis, guided interviews, an analysis of secondary data together with control group comparison, case studies, and focus group analysis.

The AplusB-Programme focusses on innovative start-up projects which are typically technologyoriented, relatively complex or demanding in terms of the level of supervision and support needed, and of considerable significance in view of the expected impact of structural change and economic growth on the economy. These projects require a high level of continued development and supervisory support right from the beginning. Neither the scientific community, nor private incubators, are currently able to support these types of projects in this manner. The spectrum of services offered by the AplusB founder centres is geared towards meeting the needs and complementing the relevant structures of the various regions (e.g. complementing the prevailing scientific, economic, funding and startup settings).

Since its inception, the AplusB-Programme has not only made a significant contribution to raising awareness among the academic and scientific community, it has also served to stimulate the development of a number of academic start-ups. Participation in various competitions has significantly raised the programme's overall level of recognition, even at the international level. Those supervised are very satisfied with the programme and the work done by the AplusB founder centres. The latter are seen to be professional, trustworthy, and stimulating, with a high level of competence across several fields, and as representing a strong and growing network of neutral and independent partners.

From 2002 to the beginning of 2014 the founder centres helped 486 businesses get started. According to the monitoring undertaken, only $9 \%$ of these start-ups were female-led. Approximately $30 \%$ of the projects would not have been realised at all without the help of AplusB, and a further $52 \%$ would only have appeared in a much reduced form. The official business demography statistics indicate that 386 of the businesses were still operating at the beginning of 2014. According to own surveys, the start-ups were responsible for the creation of about 3,400 jobs in the period 2002 to 2015. The official Austrian employment statistics shows, that at the end of 2013, AplusB businesses employed 1,752 people. Compared with the control group, AplusB businesses are characterized by a higher survival rate, a greater impact on employment, more dynamic revenue growth, higher export rates, and more intensive research and development. These results were found to be relatively constant across regions.

In future, however, particular attention needs to be placed on the following issues: Further coordination and planning across national and regional levels, improved profiling and image development for the programme, definition of interfaces with private incubators, emphasizing the core element of 'high-end' start-ups, clarification of essential parameters and respective monitoring procedures, more precise definition of programme objectives with a view to improving adaptability to new developments, strengthening the gender-sensitivity of the current offering and facilitating more direct and comprehensive exchange among female-led start-ups at the national level.

Thus, given the above background, from today's perspective continuation of the programme clearly appears to be both useful and necessary. 


\section{Erweiterte Zusammenfassung}

Technologie- und wissensintensive Gründungen etablieren radikale Innovationen bzw. neue Organisationsformen und sind ein wesentlicher Motor des strukturellen Wandels. Sie halten aber auch die Märkte offen und forcieren die Wettbewerbsfähigkeit etablierter Unternehmen. Darüber hinaus können sie einen merklichen Beitrag zu Beschäftigung und sozialer Mobilität leisten. Mit dem Programm Academia plus Business (AplusB) wurde in Österreich in den vergangenen Jahren ein Instrument aufgebaut, das explizit Ausgründungsaktivitäten aus dem akademischen Umfeld als einen Kanal zur kommerziellen Nutzung von wissenschaftlichen Ergebnissen und/oder Kompetenzen bereits in einer frühen Phase unterstützt.

Das AplusB-Programm wird seit seinem Start durch Bundesmittel, Landesmittel und private Quellen finanziert. Der Beitrag des Bundes zur Gesamtfinanzierung liegt bei rd. einem Drittel.

\section{Finanzierung des AplusB-Programms}

\begin{tabular}{|l|c|c|c|c|c|c|c|c|c|c|}
\hline \multirow{2}{*}{} & \multicolumn{3}{|c}{ 1. Förderungsperiode 2002/07* } & \multicolumn{3}{c|}{ 2. Förderungsperiode 2007/12** } & \multicolumn{2}{c|}{$\begin{array}{l}\text { 3. Förderp. } \\
\text { 2012/17*** }\end{array}$} \\
\cline { 2 - 12 } & Plan & $\%$ & Ist & $\%$ & Plan & $\mathbf{\%}$ & Ist & \% & Plan & \% \\
\hline Bund & 18.506 .010 & 42 & 17.635 .930 & 41 & 14.640 .642 & 32 & 13.166 .250 & 32 & 16.908 .300 & 34 \\
\hline Land & 15.391 .020 & 35 & 14.613 .261 & 34 & 13.560 .275 & 30 & 12.081 .203 & 29 & 13.992 .573 & 28 \\
\hline $\begin{array}{l}\text { Eigen- } \\
\text { mittel }\end{array}$ & 10.544 .556 & 24 & 10.803 .926 & 25 & 17.090 .870 & 38 & 15.868 .588 & 39 & 18.551 .578 & 38 \\
\hline Summe & 44.441 .586 & $\mathbf{1 0 0}$ & 43.053 .117 & $\mathbf{1 0 0}$ & 45.291 .787 & $\mathbf{1 0 0}$ & 41.116 .041 & $\mathbf{1 0 0}$ & 49.452 .451 & $\mathbf{1 0 0}$ \\
\hline
\end{tabular}

Anmerkung: * 9;**8;**7 Zentren; 3. Förderungsperiode mit Planwerten. Rundungsdifferenzen nicht ausgeglichen. Quelle: Statistik Austria, Berichtswesen AplusB, Berechnung und Darstellung JR

Ziel der vorliegenden Evaluierung ist die gesamtheitliche Betrachtung der erzielten Wirkungen des AplusB-Programms nach seiner nun mehr als zehnjährigen Laufzeit. Die Evaluierung bezieht sich auf das Gesamtprogramm und nimmt keine Bewertung einzelner Zentren oder regionaler Stakeholder vor.

Die Untersuchung erfolgte auf Basis eines Multi-Ebenen-Ansatzes, welcher insbesondere zwischen der Programm-, Zentrums- und Gründungsperspektive differenzierte sowie eine Analyse aus mehreren Blickwinkeln, unter Einbindung regionaler Stakeholder, ermöglichte. Die Bewertung basiert auf Literatur- und Dokumentenauswertungen, einer Logic-Chart-Analyse, leitfadengestützten Interviews, einer Sekundärdatenanalyse mit Kontrollgruppenvergleich, Fallstudien sowie Fokusgruppen.

\section{Rahmenbedingungen von AplusB}

Die Aufmerksamkeit und das Bewusstsein für die strukturpolitische Bedeutung von Entrepreneurship und wissensintensiven, unternehmerischen (Aus-)Gründungen sind weltweit, so auch in Österreich, zuletzt stark angestiegen. In diesem Zusammenhang ist festzustellen, dass AplusB seit Bestehen wesentlich zu einer entsprechenden Sensibilisierung für Gründungen aus dem akademischen Umfeld, auf regionaler wie auf nationaler Ebene, beigetragen hat.

Das Thema kommerzielle Verwertung von Forschungsergebnissen rückt in Forschungseinrichtungen, speziell an Hochschulen, stärker in den Mittelpunkt. Die Hochschulen sind im Bereich der „Third- 
Mission" unterschiedlich intensiv engagiert. Auf Grund der zur Verfügung stehenden Ressourcen und Instrumente sind wissenschaftliche Einrichtungen nur eingeschränkt in der Lage, hochkomplexe Gründungsprojekte über einen längeren Zeitraum intensiv zu begleiten. Im Zusammenspiel mit AplusB können Hochschulen und Forschungseinrichtungen allerdings einen wesentlichen Beitrag im Bereich der Bewusstseinsbildung und Vorfeldarbeit übernehmen.

\section{Ziele und Zielgruppen von AplusB}

Ausgehend von akademischen Spin-offs aus wissenschaftlichen Einrichtungen, d.h. gegründet von Personen mit akademischem Hintergrund, wurde die Zielgruppe des AplusB-Programms mit dem Übergang in die zweite Förderungsperiode bewusst auf AkademikerInnen mit Berufserfahrung (d.h. aus einem wirtschaftlichen Umfeld) ausgeweitet. Damit wurde dem Umstand Rechnung getragen, dass potentielle GründerInnen sich nach ersten beruflichen Erfahrungen in der Privatwirtschaft bereit fühlen und entsprechend qualifiziert sind, ein Gründungsprojekt in Angriff zu nehmen. Im Hinblick auf zukünftige Entwicklungsschritte ist die klare Positionierung der AplusB-Zentren jedoch dauerhaft zu erhalten.

Abgesehen von dieser erweiterten Zielgruppendefinition wurden die wesentlichen Teilziele des Programms (z.B. dauerhafter Anstieg der Anzahl akademischer Spin-offs, Verbesserung der unternehmerischen Verwertung von Forschungsergebnissen) beim Übergang in die 3. Förderungsperiode (,AplusB 2.0“) jedoch ohne Anpassung übernommen. Auch wenn die Begrifflichkeiten im Einzelnen angepasst werden können, sind grundlegende Zielkonflikte im Programmgefüge nicht feststellbar. Das Interventionsmuster des AplusB-Programms ist konsistent und zweckmäßig.

$A p l u s B$-Gründungen sind üblicherweise technologieorientierte, komplexe, in der Betreuung über einen längeren Zeitraum aufwendige Projekte. Unterschiede im Aufwand lassen sich vor allem zwischen sogenannten Hard- und Softwaregründungen feststellen. Letztere finden sich überwiegend im Bereich der Informations- und Kommunikationstechnologien (IKT), die in unterschiedlichen Sektoren angesiedelt sein können.

Nur knapp $9 \%$ der im Rahmen des Gründungs-Monitorings ersterfassten GründerInnen sind Frauen. Der Unterstützung von Frauen als potentielle GründerInnen wurde zuletzt durch AplusB etwas höhere Aufmerksamkeit gewidmet. Frauen sind in vielen Bereichen naturwissenschaftlich-technischer Ausbildungen an Hochschulen sowie Beschäftigungen in Technologie-intensiven F\&E-Bereichen noch unterrepräsentiert. Darüber hinaus ergibt sich der Befund, dass Frauen auf Grund der vergleichsweise stark ausgeprägten, horizontalen wie vertikalen Segregation im FTI-Bereich sowie kulturell geprägter Vorstellungen und Stereotype die Möglichkeit einer Unternehmensgründung seltener in Betracht ziehen als Männer.

\section{Rolle und Positionierung von AplusB}

Das AplusB-Programm setzt bereits in sehr frühen Phasen mit seiner Betreuung von Gründungsvorhaben an. Anders als das FFG-Programm Research Studios Austria setzt AplusB nicht beim Forschungsprojekt und der Verwertung von dessen Ergebnissen an, sondern bei der Gründungsidee, die bereits von GründerInnen vertreten wird. Die Zentren bieten Information und Beratung in einem Umfang und einer Tiefe an, wie sie aktuell in Österreich von keinem anderen Akteur geboten werden.

Die enge Anbindung an akademische Einrichtungen ist ein Alleinstellungsmerkmal der AplusBZentren in der heimischen Unterstützungslandschaft für Gründungen. Abseits der Trägerorganisationen sind die Zentren mit regionalen Akteuren wie Standortagenturen und anderen Inkubatoren gut vernetzt. 
Mögliche Synergiepotentiale im Awareness- und Pre-Inkubationbereich werden jedoch noch nicht vollends ausgeschöpft.

Die großteils bilateralen Abstimmungen auf regionaler Ebene zwischen den relevanten Akteuren sind sehr positiv zu bewerten. Die Berücksichtigung regionaler Gegebenheiten und Bedarfe führt dazu, dass die Angebotsprofile der einzelnen Zentren voneinander abweichen.

Private Anbieter können das Angebot von AplusB ergänzen, jedoch nicht im derzeitigen Umfang ersetzen oder übernehmen. Private Initiativen haben üblicherweise nicht das Ziel und sind dafür nicht ausgestattet, Gründungen zu stimulieren (z.B. durch Bewusstseinsbildung, Pre-Inkubation) oder risikoreiche und erheblich entwicklungsbedürftige Projekte in frühen Stadien zu übernehmen. Schließlich ist anzumerken, dass private Initiativen regional sehr ungleich verteilt sind.

Die Ausgangsbasis der Gründungsvorhaben (Erfahrung der GründerInnen, Unternehmensgegenstand) sowie die unterschiedlichen Reifegrade der Gründungsideen führen zu unterschiedlich hohen Betreuungsaufwänden. Gerade deswegen ist es wichtig, dort, wo möglich, „Good-Practices“ auszutauschen und, wo notwendig, Standards festzulegen.

Die Zentren unterscheiden sich in Abhängigkeit von Angebotsprofil und Ausgestaltung (Größe, Zahl der Standorte) auch hinsichtlich der Kostenstruktur erheblich. In einzelnen Fällen wurden in jüngerer Zeit gezielte Maßnahmen mit dem Ziel von Effizienzsteigerungen und Kosteneinsparungen realisiert.

\section{Umsetzung und Monitoring von AplusB}

Das Programm wurde 2002 implementiert und zu Beginn mit neun Zentren operativ umgesetzt. 2007 ist ein Zentrum (v-start ${ }^{1}$ ) aus dem Programm ausgeschieden, 2013 wurde ein Ansuchen auf Weiterförderung $\left(\mathrm{BCCS}^{2}\right)$ abgelehnt. 2015 werden somit in sieben regionalen AplusB-Zentren akademische Unternehmensgründungen und Spin-offs unterstützt und gefördert. Das sind accent NÖ, build! Kärnten, CAST Tirol, INiTS Wien, Science Park Graz, tech2b OÖ und ZAT Leoben. Die Anzahl der Beschäftigten liegt je nach Zentrumsgröße zwischen drei (ZAT) und fünfzehn (INiTS) MitarbeiterInnen.

Im Zuge der Implementierung von „AplusB 2.0“ wurde das Leistungsprofil überarbeitet und geschärft. Die Bandbreite reicht nun von Öffentlichkeitsarbeit, Stimulierungs- und Awareness-Maßnahmen, individueller Beratung und Coaching, dem Bereitstellen von Infrastruktur bis zur Netzwerkaktivitäten und der Finanzierung und Unterstützung von Internationalisierungsprojekten. Während sogenannte „MUSS-Module“ im Wesentlichen das bisherige Leistungsspektrum abdecken, widmen sich „KANNModule“ Aspekten wie Finanzierung, Internationalisierung oder der Alumni-Betreuung.

AplusB-Zentren bewältigen einen wesentlichen Teil der Abstimmung zwischen regionalen Bedarfen, der Politik und dem Gesamtprogramm AplusB. Die regionale Zusammenarbeit zwischen Zentren und Netzwerk- und Kooperationspartnern wird von den Stakeholdern auf regionaler Ebene allgemein als sehr gut eingeschätzt.

Die befragten GründerInnen bestätigen die Zufriedenheit mit dem Angebot und den Leistungen der AplusB-Zentren. Die AplusB-Zentren werden als vertrauenswürdiger Partner mit Kompetenz in mehreren Themenfeldern, hoher Professionalität, Glaubwürdigkeit und Motivation und einem starken und wachsenden Netzwerk wahrgenommen, der für Unabhängigkeit ohne Eigeninteresse steht. Potentielle GründerInnen werden von Beginn an intensiv begleitet, ihre spezifischen Probleme individuell geklärt

v-start (1.7.2005-30.6.2010) wurde mit 01.07.2010 in die Wirtschafts-Standort Vorarlberg GmbH (WISTO) integriert und fungiert dort weiterhin als Gründungszentrum für High-Tech-Gründungen.

BCCS-Business Creation Center Salzburg, Salzburg (01.07.2005 bis 31.12.2014). 
und an ihr persönliches Ziel geführt. Die AplusB-Zentren regen eine sehr detaillierte Ausarbeitung und oftmalige Reflexion der Geschäftspläne an. Die Netzwerk- und Vermittlungsarbeit der Zentren wird als besonders wertvoll angesehen, ebenso die Möglichkeit des Austausches mit anderen GründerInnen. Darüber hinaus wurden auch Schulungsangebote zu geistigem Eigentum und Markenschutz positiv hervorgehoben.

Das Programmmanagement bei der FFG ist sehr schlank aufgebaut und deckt die Grundfunktionen ab. Die AplusB-Plattform hat sich als mittlerweile als gut frequentiertes Instrument etabliert. Sie unterstützt den Austausch zwischen den AplusB-Zentren, aber auch mit dem Programmmanagement. Der Zentrenübergreifende Austausch zwischen GründerInnen wird gegenwärtig nicht offensiv verfolgt.

Das AplusB-Programm verfügt über ein umfangreiches Berichts- und Monitoringsystem und war diesbezüglich anderen Förderungsprogrammen von Anbeginn an voraus. Die Evaluierungsarbeiten und damit einhergehenden Auswertungen haben allerdings auch Spielräume für Vereinfachungen und Verbesserungen deutlich gemacht. Dies betrifft unter anderem den Bereich der mit den Programmzielen korrespondierenden Ergebnisindikatoren.

\section{Wirkungen von AplusB}

AplusB trug und trägt nach Aussagen regionaler Stakeholder und Partner in hohem Ausmaß zur Steigerung der Awareness bezüglich Selbstständigkeit bei. AplusB wird als starker Treiber in der Gründerszene wahrgenommen, was sich etwa dadurch zeigt, dass die erfolgreichsten Gründungen der letzten Jahre eben oft mit AplusB in Verbindung stehen.

International fanden die Leistungen von AplusB ebenfalls große Anerkennung. So wurde INiTS 2014 von der schwedischen Forschungsinitiative UBI Index in das Ranking der „Global Top 25“ universitären Inkubatoren aufgenommen, wo das AplusB-Zentrum weltweit den elften Platz und europaweit den dritten Platz belegt. Der Verein Österreichisches Inkubatorennetzwerk AplusB ergänzt die Kommunikation nach außen, auch wenn es hier noch Anpassungsbedarfe, etwa für den internationalen Auftritt, gibt.

Die Sensibilisierungs- und Awareness-Maßnahmen der Zentren erreichen, explizit (durch eigene Veranstaltungen, z.B. Business Angel Day) sowie implizit (z.B. Mitarbeit bei Lehrveranstaltungen an Hochschulen) jährlich AkademikerInnen in den Trägerorganisationen sowie anderen wissenschaftlichen Einrichtungen. Die Beteiligung an unterschiedlichen Wettbewerben hat den Bekanntheitsgrad des Programms selbst stark erhöht. Die Leistungen des AplusB-Programms zum Aufbau von Kooperationsbeziehungen, zum Austausch innerhalb der Gründungs-Community und zur Identifikation von notwendigen Expertisen sind positiv zu bewerten. Wie insbesondere die Fallstudien zeigen, lässt sich die AplusB-Unterstützung nur schwer durch ein hohes persönliches Engagement sowie ausgeprägte private Netzwerke aufwiegen. Um die Maßnahmenvielfalt und deren Ergebnisse künftig besser einschätzen zu können, bedarf es nunmehr eines erweiterten, zahlenbasierten und einheitlich definierten Monitoringsystems.

Das Programm AplusB und die Arbeit der AplusB-Zentren werden von den betreuten Gründungen sehr positiv bewertet. Die AplusB-Zentren werden als vertrauenswürdiger Partner mit Kompetenz in mehreren Themenfeldern, hoher Professionalität, Glaubwürdigkeit und Motivation und einem starken und wachsenden Netzwerk wahrgenommen, der für Unabhängigkeit ohne Eigeninteresse steht. Potentielle GründerInnen werden von einer Frühphase der Entwicklung an intensiv begleitet.

In Zahlen gefasst ergeben sich folgende Befunde (siehe dazu auch nachfolgende Tabelle): 
- Im Schnitt führen in den AplusB-Zentren pro Jahr rd. 800 evaluierte Ideen zu ca. 500 Erstgesprächen. Nach Prüfung durch den Projektbeirat werden dann ca. 50 Projekte pro Jahr in die Inkubationsprozesse der Zentren aufgenommen.

- Zwischen 2002 und Anfang 2014 wurden lt. Gründungs-Monitoring 486 Unternehmen gegründet. Der Anteil der ersterfassten Gründerinnen beträgt dabei lediglich rd. $9 \%$.

Rd. $30 \%$ der Vorhaben wären ohne das Programm nicht in Angriff genommen worden, weitere $52 \%$ lediglich in reduzierter Form. In Abgleich mit der offiziellen Statistik zur Unternehmensdemographie zeigt sich, dass bis Anfang 2014 noch 386 dieser Unternehmen wirtschaftlich aktiv waren.

- Zwischen 2002 und 2015 wurden lt. Eigenerhebung der AplusB-Zentren zumindest 3.400 Arbeitsplätze geschaffen. Diese Anzahl beinhaltet sowohl Abschätzungen für übernommene Unternehmen und Beschäftigte auf internationaler Ebene als auch vereinzelt WerkvertragsnehmerInnen und einbezogene Non-AplusB-Beschäftigte. Etwaige Beschäftigungsrückgänge bzw. Ausfälle wurden bei der AplusB-Zentren nur eingeschränkt berücksichtigt. Vor diesem Hintergrund und dem Ziel einer einheitlichen, definitorischen Abgrenzung wird eine Revision der Erfassung wesentlicher Wirkungsindikatoren als wesentlich erachtet.

In direktem Abgleich mit der offiziellen Beschäftigungsstatistik zeigt sich, dass sich der Stand der Arbeitsplätze in unabhängigen AplusB-Unternehmen in Österreich bis inkl. 2013 auf 1.752 belief, davon waren 1.665 unselbstständig beschäftigt (dies entspricht 950 Beschäftigten in aktiven $A p$ lusB-Unternehmen im Jahr 2010 und 382 Beschäftigten im Jahr 2007).

- Im Vergleich zu einer Kontrollgruppe (64 \%) weisen AplusB-Gründungen eine deutlich höhere Überlebensrate $(90 \%)$ nach dem fünften Jahr des Bestehens auf. Zudem erreichen AplusBGründungen eine vergleichsweise höhere Beschäftigungswirkung (80\% höher als die Kontrollgruppe nach dem fünften Jahr).

Ein interessanter Aspekt ist zudem die Tatsache, dass sich die regional unterschiedlichen Gegebenheiten sowohl bei den AplusB-Gründungen als auch bei der Kontrollgruppe nur unwesentlich in der Überlebensfähigkeit der gegründeten Unternehmen niederschlagen.

- Weiter ist festzustellen, dass AplusB-Unternehmen zwar mit einer geringeren Umsatzgröße ins Unternehmertum starten, sie die Kontrollgruppe aber aufgrund einer höheren Dynamik im Durchschnitt im vierten Jahr nach der Gründung überholen.

Im Einklang mit der Definition der Zielgruppe weisen mehr als die Hälfte der AplusB-Gründungen bis zum fünften Jahr F\&E-Aktivitäten auf. Trotz ihrer vergleichsweise geringen Zahl entfällt rd. ein Fünftel der F\&E-Aufwendungen und -Beschäftigten aller Jungunternehmen bis zum fünften Jahr auf AplusB-Gründungen. Ein ähnliches Bild zeigt sich im Zusammenhang mit der erfolgreichen Bewerbung bei Förderungsprogrammen.

Auf Basis der erfassten Zahlen lässt sich daher ein sehr positives Resümee zu den Ergebnissen und Wirkungen des Programms ziehen. 


\section{AplusB-Ergebnisse und Wirkungen im Überblick}

\begin{tabular}{|c|c|c|c|c|c|c|c|}
\hline \multicolumn{7}{|c|}{ Finanzierung 2002-2017*, gesamt } & Quellen \\
\hline \multicolumn{5}{|l|}{ Bund } & \multicolumn{2}{|c|}{ 47,7 Mio. $(36 \%)$} & \multirow[t]{3}{*}{ bmvit, $F F G$} \\
\hline \multicolumn{5}{|l|}{ Länder } & \multicolumn{2}{|c|}{ 40,7 Mio. $(30 \%)$} & \\
\hline \multicolumn{5}{|l|}{ Eigenmittel } & \multicolumn{2}{|c|}{ 45,2 Mio. (34\%) } & \\
\hline \multicolumn{7}{|c|}{ Selektionsprozesse, Ø 2014} & \\
\hline \multicolumn{5}{|l|}{ Anzahl evaluierte Ideen } & \multicolumn{2}{|c|}{ ca. 800} & \multirow{3}{*}{$\begin{array}{l}\text { AplusB Moni- } \\
\text { toring }\end{array}$} \\
\hline \multicolumn{5}{|l|}{ Anzahl Erstgespräche } & \multicolumn{2}{|c|}{ ca. 500} & \\
\hline \multicolumn{5}{|c|}{ Anzahl aufgenommene Projekte } & \multicolumn{2}{|c|}{ ca. 50} & \\
\hline \multicolumn{7}{|c|}{ Schlüsselindikatoren bei Gründungen } & \\
\hline \multicolumn{5}{|c|}{ Anzahl Gründungen, gesamt } & $2002-2014$ & 486 & \multirow{2}{*}{$\begin{array}{c}\text { Evaluierung, } \\
\text { Statistik } \\
\text { Austria }\end{array}$} \\
\hline \multicolumn{5}{|c|}{ Gründungen, die ohne AplusB-Unterstützung nicht zustande gekommen wären } & $2002-2014$ & $30 \%$ & \\
\hline \multicolumn{5}{|c|}{ Anteil von Frauen unter AplusB-GründerInnen } & $2002-2014$ & $9 \%$ & $\begin{array}{l}\text { AplusB- } \\
\text { Monitoring }\end{array}$ \\
\hline \multicolumn{5}{|c|}{$\begin{array}{l}\text { Anzahl geschaffene Arbeitsplätze, gesamt } \\
\text { (inkl. übernommene Unternehmen und Beschäftigung international }{ }^{3} \text { ) }\end{array}$} & $2002-2015$ & 3.400 & $\begin{array}{l}\text { AplusB- } \\
\text { Zentren }\end{array}$ \\
\hline \multicolumn{5}{|c|}{ Anzahl aktiver (,überlebender) AplusB-Unternehmen gesamt in Österreich } & 2013 & 386 & \multirow{4}{*}{$\begin{array}{c}\text { Evaluierung, } \\
\text { Statistik } \\
\text { Austria }\end{array}$} \\
\hline \multicolumn{5}{|c|}{ Anzahl Beschäftigte bei aktiven AplusB-Unternehmen in Österreich } & 2013 & 1.752 & \\
\hline \multicolumn{5}{|c|}{$\begin{array}{l}\text { Anzahl unselbständig Beschäftigte bei aktiven AplusB-Unternehmen in Öster- } \\
\text { reich }\end{array}$} & 2013 & 1.665 & \\
\hline \multicolumn{5}{|c|}{ Anzahl von in Unternehmen im dritten Jahr geschaffenen Arbeitsplätzen } & $\varnothing 2014$ & ca. 200 & \\
\hline \multicolumn{5}{|c|}{$\begin{array}{l}\text { Anzahl Unternehmen, die bei FFG und aws komplementäre Förderung einge- } \\
\text { worben haben }\end{array}$} & 2013 & 378 & $\begin{array}{l}\text { Evaluierung, } \\
\text { aws, FFG }\end{array}$ \\
\hline \multicolumn{8}{|c|}{ Entwicklung im 5. Jahr nach der Gründung } \\
\hline & Überleben & $\begin{array}{l}\text { Anzahl } \\
\text { Mitarbeit- } \\
\text { erInnen }^{4}\end{array}$ & $\begin{array}{l}\text { Umsatz } \\
(\text { in TSD } €)\end{array}$ & $\begin{array}{l}\text { Export- } \\
\text { intensität }\end{array}$ & $\begin{array}{l}\text { F\&E- } \\
\text { Neigung } \\
\text { Hochtechn. } \\
\text { Dienstleist. }\end{array}$ & $\begin{array}{l}\text { F\&E- } \\
\text { Neigung } \\
\text { Mittel- und } \\
\text { Hochtechn. }\end{array}$ & \multirow[t]{3}{*}{$\begin{array}{c}\text { Evaluierung, } \\
\text { Statistik } \\
\text { Austria }\end{array}$} \\
\hline AplusB-Unternehmen & $87 \%$ & 8,9 & 615 & $43 \%$ & $56 \%$ & $90 \%$ & \\
\hline Kontrollgruppe & $64 \%$ & 4,5 & 434 & $16 \%$ & $1 \%$ & $58 \%$ & \\
\hline
\end{tabular}

Anmerkung: * 3. Förderungsperiode mit Planwerten

Quelle: Berechnung und Darstellung JR

3 Es entstanden weitere Unschärfen in der Erfassung dadurch, dass WerkvertragsnehmerInnen und non-AplusB-Beschäftigte einbezogen wurden bzw. die Zahlen nicht in Hinblick auf etwaige aktuelle Beschäftigungsrückgänge oder Ausfälle bereinigt wurden.

4 Der Vergleich bezieht sich auf Unternehmen mit einem Mindestumsatz von $30.000 €$ pro Jahr. 


\section{Schlussfolgerungen und Empfehlungen}

Aus der heutigen Sicht erscheint die Weiterführung des Programms unter Berücksichtigung der folgenden Empfehlungen als sinnvoll und notwendig:

- Die gemeinsame Abstimmung und Konzeption auf nationaler Ebene in Kombination mit der Arbeit mit wissenschaftlichen Einrichtungen und Fördereinrichtungen vor Ort stellen eine Besonderheit und Stärke des Programms AplusB dar und sollten beibehalten werden. Die AplusB-Zentren stellen eher kleine, jedoch hochflexible Einheiten dar. Die mit der aktuellen Förderungsperiode bekräftigte Entscheidung, zumindest regional konzentrierte Einheiten in einer kritischen Größe zu unterstützen, hat sich als sinnvoll herausgestellt.

- Einzelne AplusB-Zentren sehen den Bedarf, den Bereich nicht-akademischer innovativer Gründungen in Zukunft intensiver zu verfolgen, um auf regionale Potentiale noch besser reagieren zu können. Soweit es vom Bund gefördert wird, müssen auch bei der Erweiterung des Horizonts der einzelnen Zentren die Wurzeln bei „High-End“-Gründungen im Umfeld wissenschaftlicher Einrichtungen im Fokus bleiben.

- Die AplusB-Plattform soll in der Zukunft weiterhin unterstützt, ihre Rolle beim Transfer von „Good Practices“ in Hinblick auf erfolgreiche Modelle und Ansätze zu Effizienzsteigerungen verstärkt sowie bei der Abstimmung von Potentialen einer funktionalen Arbeitsteilung zwischen den Zentren gefestigt werden.

- Dem Bedürfnis regionaler Förderungsstellen und Stakeholder, sich in regelmäßigen Abständen direkt mit dem Programmmanagement sowie dem bmvit abzustimmen, kann in Form von periodischen Treffen relativ einfach nachgekommen werden. Beispielgebend können hierfür die laufenden Treffen mit Stakeholdern in Zusammenhang mit dem COMET-Programm sein.

- Private Initiativen und AplusB-Zentren weisen grundsätzlich komplementäre Foki und Angebote auf. Es wird daher die Darstellung eines klaren Profils sowie die Definition von klaren Nahtstellen zu privaten Initiativen - insbesondere auf der Ebene der einzelnen Zentren - empfohlen, die nach außen kommuniziert werden.

- Der Einsatz von der Inkubation vor- und nachgelagerten Aufgaben (Bewusstseinsbildung, AlumniBetreuung) der AplusB-Zentren ist im Verhältnis zur Inkubationstätigkeit zu bewerten. Es wird daher eine klare Festlegung in den angestrebten Outputs in Abstimmung mit den von den Trägerorganisationen und regionalen Fördereinrichtungen erbrachten Leistungen empfohlen.

- Die Zusammenarbeit zwischen Spin-Offs und Industrieunternehmen („Industriekooperationen“) nimmt an Bedeutung zu. Hier fehlt es gegenwärtig an klaren Zielen. Man kann direkt an jüngere Initiativen der Industrie mit Fokus auf Start-ups anknüpfen.

- Der wachsenden Bedeutung von Geschlechtervielfalt und Diversität und damit auch einer Erhöhung des Potentials von Frauen als GründerInnen ist durch Verbesserung bei gendergerechten Angeboten, die in einigen Zentren bereits existieren, Rechnung zu tragen.

- Es wird angeregt, Möglichkeiten des direkten, Zentren-übergreifenden Austauschs von GründerInnen auf der nationalen Ebene zu unterstützen und pflegen.

- In Hinblick auf das Berichtswesen und das laufende Monitoring werden folgende Weiterentwicklungen angeregt: Wo dies sinnvoll ist, sollte die Dokumentation in der Form eines Monitoringsystems durchgeführt werden, das mit den gesetzten Zielen korrespondierende, nachvollziehbare Indikatoren umfasst. Des Weiteren wird eine Weiterentwicklung des Gründungs-Monitoring empfohlen. Das Monitoring differenziert nicht ausreichend zwischen den antwortenden GründerInnen und den erfassten Einheiten des Gründungsvorhabens (in weiterer Folge: Unternehmen). In Folge dessen ist eine Beobachtung der betreuten Fälle nach der Gründung nur sehr eingeschränkt möglich. 


\section{Inhaltsverzeichnis}

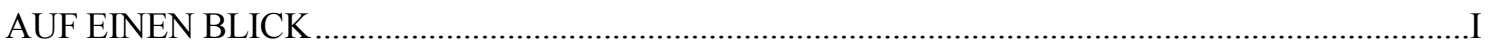

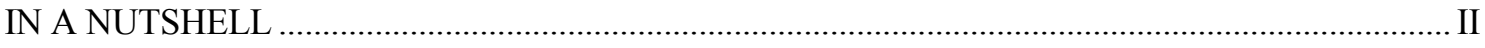

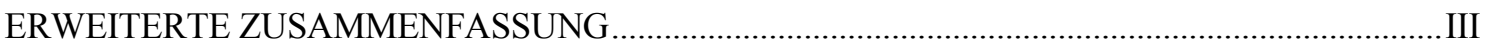

$1 \quad$ HINTERGRUND UND HERANGEHENSWEISE .........................................................

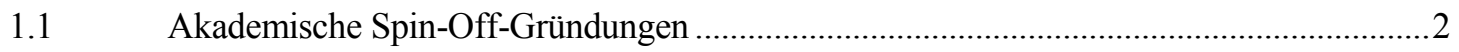

1.2 Entwicklungen und Herausforderungen ..........................................................................

$1.3 \quad$ Ziele und Methodik der Evaluierung .............................................................................

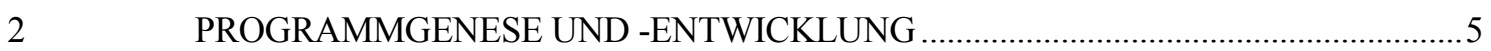

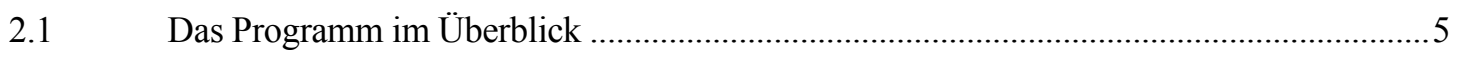

$2.2 \quad$ Mission und Ziele ...........................................................................................

$2.3 \quad$ Bewertung von Zielsetzung und Konzeption ................................................................. 10

3 PROGRAMMUMSETZUNG DURCH DIE ZENTREN …………............................... 14

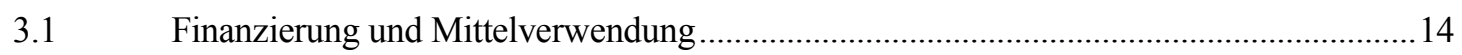

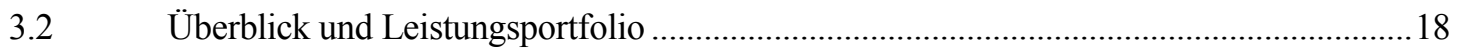

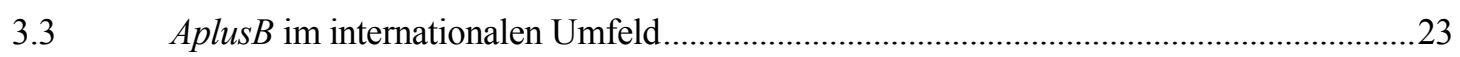

3.4 Bewertung der entwickelten Strukturen und Umsetzung ..............................................27

4 STRUKTUR UND CHARAKTERISTIKA DER APLUSB-GRÜNDUNGEN ..................32

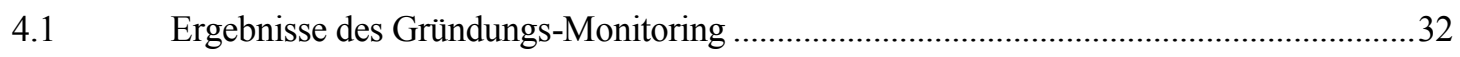

4.1.1 Aufnahme und Realisierung von Gründungsvorhaben ...................................................33

4.1.2 Strukturmerkmale der AplusB-Gründungsvorhaben ............................................................

4.1.3 Hintergründe der Entscheidung für eine Gründung im AplusB-Zentrum ............................38

4.1.4 Die Wissens- und Qualifikationsbasis der beobachteten AplusB-Gründungen ...................40

4.1.5 Herausforderungen und erste Erfahrungen der AplusB-GründerInnen...............................43

4.1.6 Verhaltensadditionalität - Wie hätten GründerInnen ohne AplusB gehandelt?..................46

4.2 Ausgründungsanlässe und Typologien von GründerInnen ................................................47

4.2.1 Typologien in Gründungsvorhaben ..............................................................................4

4.2.2 Frauen und Gründungen.............................................................................................. 50

5 WIRKUNG UND WIRKUNGSWEISE DES PROGRAMMS APLUSB UND DER GEFÖRDERTEN ZENTREN ............................................................................................53

5.1 Herausforderungen und Mehrwert des Programms am Beispiel ausgewählter Fallstudien

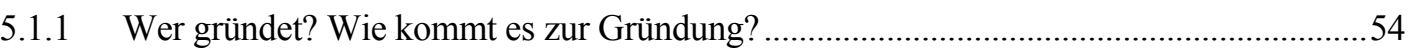

5.1.2 Wo liegen die gemeinsamen Herausforderungen in der Gründung und in den

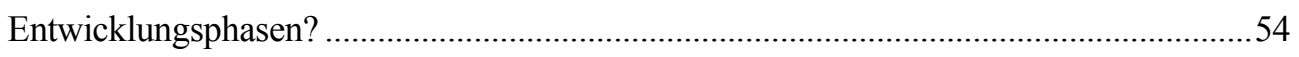

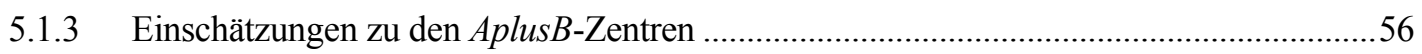

5.1.4 Zusammenfassende Beobachtung aus den Fallstudien ....................................................58 
5.2 Quantitative Beurteilung der ökonomischen Wirkungen des Programms AplusB anhand eines Vergleichs von AplusB-Gründungen mit einer Kontrollgruppe. ..............................58

5.2.1 Quantitative Analyse der Wirkung auf Überleben, Wachstum, Beschäftigung und F\&E

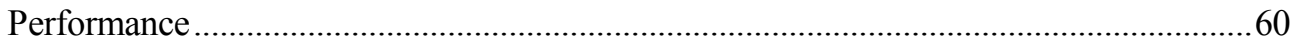

5.2.2 Die Inanspruchnahme komplementärer Förderung durch AplusB-Gründungen Verortung in der Förderungslandschaft

5.3 Beurteilung in Hinblick auf die Angemessenheit und den Mehrwert der erbrachten AplusB Leistungen sowie die statistisch nachvollziehbaren ökonomischen Wirkungen .72

Fallstudien. 


\section{Abbildungsverzeichnis}

Abbildung 1

Abbildung 2

Abbildung 3

Abbildung 4

Abbildung 5

Abbildung 6

Abbildung 7

Abbildung 8

Abbildung 9

Abbildung 10

Abbildung 11

Abbildung 12

Abbildung 13

Abbildung 14

Abbildung 15

Abbildung 16

Abbildung 17

Abbildung 18

Abbildung 19

Abbildung 20

Abbildung 21

Abbildung 22

Abbildung 23

Abbildung 24

Abbildung 25

Abbildung 26

Abbildung 27

Abbildung 28

Abbildung 29

Abbildung 30

Abbildung 31

Abbildung 32
Wirkungsdimensionen von Unternehmensgründungen 1

Logic Chart des AplusB-Programms. ... 9

Finanzierungsstruktur der AplusB-Zentren nach Finanzierungsquellen (in \%).......... 16

Gesamtkosten nach Modulen (in \%), 3. Förderungsperiode zwischen 2012-2017 ..... 17

Leistungen der AplusB-Zentren 22

Gesamtzahl der bisher im Rahmen des Gründungs-Monitorings erfassten Fragebögen32 Aufgenommene Gründungsvorhaben in den Jahren 2002-2015, absolut .....

Entwicklung der in den einzelnen AplusB-Zentren aufgenommenen Gründungsvorhaben 34

Entwicklung der Gründungsvorhaben und -projekte, gesamt, 2002-2015 . 35

Anteil der Gründungsvorhaben, die planmäßig die Inkubationsphase abgeschlossen haben (in \%). 35

Verteilung nach Gesellschaftsformen der AplusB-Gründungen (in \%) 36

Branchenstruktur der in der offiziellen Statistik erfassten AplusB-Gründungen (in \%)37

Struktur der im Rahmen des Gründungs-Monitorings registrierten Ersterfassungen, nach Branchen und Technologiefeldern (in \%). 38

Persönliche Motive der potentiellen AplusB-GründerInnen (in \%)

Bedeutung unterschiedlicher Wege, auf denen die GründerInnen vom AplusBZentrum bzw. Programm erfahren haben (in \%).

Erwartungen der GründerInnen in Hinblick auf die Unterstützung im AplusB-Zentrum (in $\%$ )

Für die Initiierung und Gründung von $A p l u s B$-Fällen ,unverzichtbare“ Faktoren ..... 41

Verteilung nach höchsten abgeschlossenen Ausbildungen ....................................... 41

Verteilung nach höchsten abgeschlossenen Ausbildungen

Nutzung und Entwicklung von Patenten in den gegründeten Unternehmen, 2002-201343

Hemmnisse bzw. Herausforderungen ...

Finanzierungsquellen der AplusB-GründerInnen (in \%).

Schwierigkeiten im Gründungsprozess (Anteil in \%) in Deutschland ....................... 45

Bedenken im Gründungsprozess (Anteil in \%) ....

Änderungen, die die AplusB-GründerInnen aufgrund der gemachten Erfahrungen im Nachhinein vorgenommen hätten (in \%).

Verknüpfung der eingesetzten Register und Sekundärdatenquellen..

Jeweils bis zu den Bezugsjahren 2006 bis 2013 kumulierte Zahl.

Branchenabdeckung einzelner Gründungskohorten in der Programmlaufzeit, absolut60 Überlebensraten der AplusB-Gründungen im Jahr 2007 nach drei Jahren 61

Überlebensraten österreichischer Gründungen (in \% der Gründungsfälle). .62

Langfristige Entwicklung der Überlebensraten österreichischer Gründungen...

Überlebensraten von Gründungen im Jahr 2008 (in \% der Gründungsfälle) .63 
Abbildung 33 Beschäftigungsentwicklung der Gründungskohorten ab 2007 ....................................63

Abbildung 34 Beschäftigungsentwicklung der Gründungskohorten ab 2007 ...................................64

Abbildung 35 Entwicklung der durch Gründungengeschaffenen Beschäftigung.................................65

Abbildung 36 Summe der Beschäftigten in Österreich jeweils aller (noch) aktiven und (noch) unabhängigen AplusB-Unternehmen in den Jahren 2007-2013 ...................................66

Abbildung 37 Umsatzentwicklung der Gründungskohorten ab 2007 (in Tsd. €) ................................67

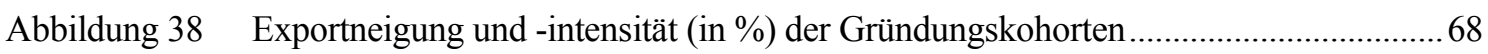

Abbildung 39 Forschungsneigung und -intensität (in \%) der Gründungskohorten ...............................68

Abbildung 40 Gegenüberstellung des Anteils der AplusB-Unternehmen an den Gründungskohorten und der Anteile am Forschungsgeschehen im Bereich dieser Gründungskohorten ....69

Abbildung 41 Inanspruchnahme von Förderungen der aws und FFG ............................................... 70

Abbildung 42 Gesamte an identifizierte AplusB-Gründungen sowie an Gründungen aus der Kontrollgruppe (2003 bis 2013) vergebene FFG-Förderungsmittel ............................. 71

Abbildung 43 Bewilligte Fördersumme bzw. geförderte Projekte........................................................ 72

\section{Tabellenverzeichnis}

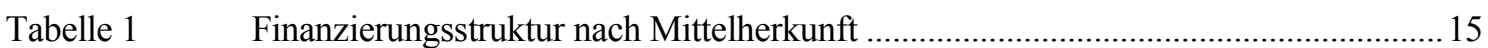

Tabelle 2 Verteilung und Zuordnung der Plankosten zur Inkubation nach Modulen ...................18

Tabelle $3 \quad$ Übersicht Zentren, Stand 2015 ............................................................................... 19

Tabelle $4 \quad$ Übersicht Gesellschafter, Verteilung 2015 _..................................................................20

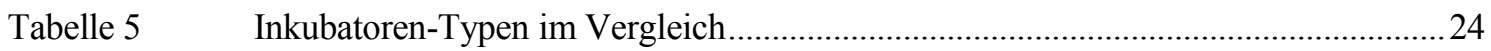

Tabelle 6 Positionierung ausgewählter internationaler Inkubatorenprogramme ..........................25

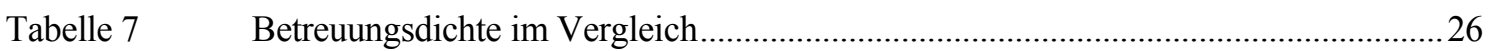

Tabelle $8 \quad$ Aufwendungen pro Gründungen im Vergleich ..........................................................2

Tabelle 9: Anzahl der Studierenden an naturwissenschaftlich-technischen Studien ...................50

Tabelle 10: $\quad$ Frauenanteil am wissenschaftlichen Personal in F\&E nach Sektoren ...........................51

Tabelle 11 Die Fallstudienbeispiele im Überblick.........................................................................53 


\section{Hintergrund und Herangehensweise}

Die Rolle von innovativen Unternehmensgründungen bei der dynamischen Entwicklung einer Wirtschaft hat in den letzten Jahren zunehmend an Bedeutung gewonnen (,entrepreneurial society ${ }^{\left({ }^{65}\right.}$ ).

Das wirtschaftspolitische Interesse an Unternehmensgründungen kann an mehreren Wirkungsdimensionen (siehe Abbildung 1) festgemacht werden. Eine hohe Bedeutung wird dabei u.a. den Wettbewerbseffekten durch das Entstehen von Neugründungen, Beschäftigungswirkungen durch die Schaffung neuer Arbeitsplätze sowie einem allgemeinen Strukturwandel auf Basis von Innovation und technologischer Entwicklung beigemessen. Insbesondere bei qualitativ hochwertigen Gründungen werden gute Wachstumsperspektiven erwartet ${ }^{6}$.

Abbildung 1 Wirkungsdimensionen von Unternehmensgründungen

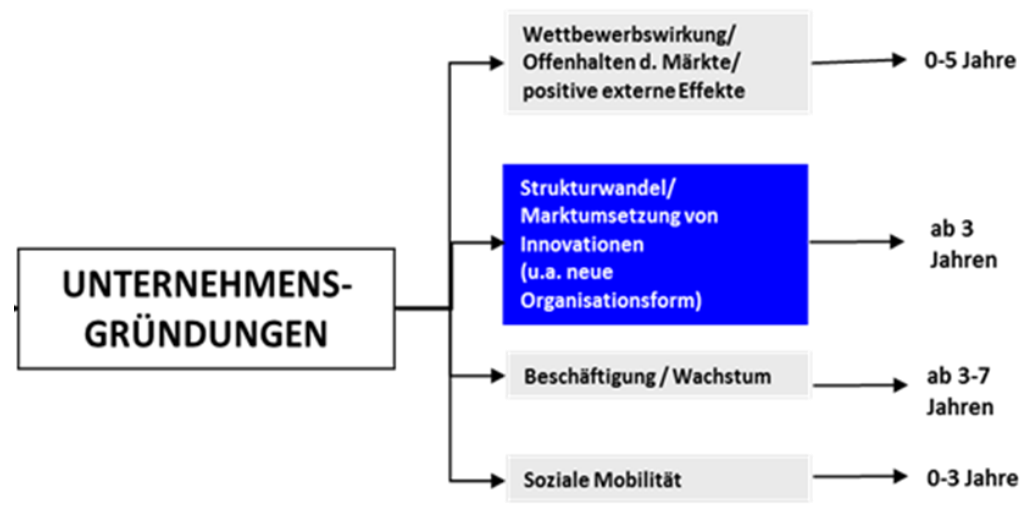

Quelle: Darstellung JR

Von der Politik wird die Stimulierung und Unterstützung von jungen, technologie- und wissensintensiven (High-Tech-) Gründungen sowie schnell wachsenden Unternehmen („Gazellen“) verstärkt vorangetrieben. Erhöhte Aufmerksamkeit wird dabei akademischen Spin-off-Gründungen - als Subsegment der High-Tech-Gründungen - zuteil.

Die österreichische FTI-Strategie 2011 hat sich zum Ziel gesetzt, die Zahl der wissens- und forschungsintensiven Gründungen bis zum Jahr 2020 um jährlich durchschnittlich $3 \%$ zu steigern. Die Förderungslandschaft im Bereich der innovativen Gründungen (im Umfeld von Hochschulen) wurde deutlich ausgebaut und verbessert, vor allem durch die Schaffung gemeinsamer Forschungszentren von Wissenschaft und Wirtschaft (Kompetenzzentren), Unterstützungsleistungen beim Technologietransfer sowie teils maßgeschneiderte Angebote an begleitenden Aktivitäten und Coaching, etwa in Richtung einer verstärkten wirtschaftlichen Verwertung von akademischem Know-how oder dem Umgang mit Schutz- und Eigentumsrechten (Intellectual Property Rights, IPR).

Eine zentrale Kraft in diesem Bereich stellt das Academia-plus-Business-(AplusB)-Programm dar. Das Programm versteht sich als zielgruppenspezifische Gründungsaktion zur Förderung von Ausgründungsaktivitäten aus Hochschulen und außeruniversitären Forschungseinrichtungen als Kanal zur

Vgl. Audretsch, D. (2009): The entrepreneurial society. In: Journal of Technology Transfer, 34, 245-254.

OECD (2010): The OECD Innovation Strategy. Getting a Head Start for Tomorrow. Paris; BMWF, BMVIT, BMWFJ (2013):

Österreichischer Forschungs- und Technologiebericht 2013; Shane, S. (2009): Why encouraging more people to become entrepreneurs is bad public policy. In: Small Business Economics, 33, 141-149. 
kommerziellen Nutzung von wissenschaftlichen Ergebnissen und/oder Kompetenzen. Derzeit werden österreichweit in acht AplusB-Zentren (sieben, seit Januar 2015) akademische Unternehmensgründungen und Spin-offs gefördert und unterstützt.

\subsection{Akademische Spin-Off-Gründungen}

Unter akademischen Spin-off-Gründungen werden Unternehmensgründungen verstanden, deren primärer Zweck die kommerzielle Verwertung des an Hochschulen und öffentlichen Forschungseinrichtungen produzierten neuen Wissen bzw. einer dort hervorgebrachten, neuen technologischen Entwicklung am Markt ist. Gegründet wird vorwiegend von StudentInnen, AbsolventInnen oder Angestellten von Hochschulen und Forschungseinrichtungen. Gründungsvorhaben, die von Personen ohne akademischen Hintergrund durchgeführt, jedoch direkt auf neues Wissen bzw. Technologien von einer Hochschule bzw. Forschungseinrichtung aufbauen, werden in die Betrachtung von akademischen Gründungen oftmals miteinbezogen. ${ }^{7}$

Akademische Spin-Off-Gründungen sehen sich eingehend mit dem Innovationsgrad hoher Komplexität, unklarer Entwicklungspfade und der damit verbundenen Unsicherheit sowie strukturellen Herausforderungen konfrontiert. Zu Letzteren zählen etwa fehlende Managementerfahrung, der Mangel an qualifiziertem Personal oder der Zugang zu Finanzierungsquellen (Fremd-, Risikokapital).

Die Stimulierung von akademischen Gründungen aus einem wissenschaftlichen Umfeld heraus sowie deren Unterstützung in der Definition und Entwicklung kann durch konventionelle Formen der Gründungsförderung nicht hinreichend bewältigt werden. Eingehend mit einem sich wandelnden Bild von Karrieremobilität und lebenslangem Lernen löst sich das Verständnis von innovativen akademischen Gründungen zunehmend von der strikten Vorstellung eines unmittelbaren Absprungs von der Hochschulforschung und -karriere.

Unterschiedliche Vorgeschichten, Reifegrade der Vorhaben (Idee vs. fertiges Business-Modell), Gründertypen (ForscherInnen vs. „Entrepreneur"), strukturelle Merkmale (Branche, Technologie) sowie die F\&E- und Innovationsorientierung erfordern in der Förderung und Begleitung jeweils eine intensive Auseinandersetzung mit dem Einzelfall. In Hinblick auf Herausforderungen und Unterstützungsbedarf lassen sich nur sehr beschränkt für die Förderpraxis nutzbar Typen ableiten (siehe dazu Abschnitt 4.2)

\subsection{Entwicklungen und Herausforderungen}

Akademische Ausgründungen auf Basis von wissenschaftlicher Forschung sind heute nicht nur ein wichtiger Kanal des Technologietransfers aus Universitäten und Forschungseinrichtungen, sondern werden oft als Beleg für die hohe Qualität von Forschung bzw. Lehre an der Mutterorganisation angesehen und scheinen dementsprechend auch in den Monitoring- und Berichtsformaten auf.

Das Hochschulsystem in Österreich hat sich in jüngster Vergangenheit deutlich weiterentwickelt. Dies betrifft etwa die Differenzierung unterschiedlicher Hochschultypen (Universität, Fachhochschule) sowie die zunehmende Eigenverantwortung (UOG93) bzw. Autonomie der Universitäten (UOG02). Diese Veränderung im österreichischen Hochschulwesen brachte eine grundlegende Neuausrichtung der universitären Steuerungs- und Lenkungsmechanismen mit sich, begleitet von erhöhten Anforderungen hinsichtlich längerfristiger Strategie- und Profilbildung sowie steigenden Anforderungen hin-

Vgl. OECD (2000): Analytical Report on High Tech Spin-offs, Paris (DSTI/STP/TIP(2000)7). 
sichtlich der Einwerbung von Drittmitteln und der Evaluierung wissenschaftlicher und wirtschaftlicher Ergebnisse. ${ }^{8}$

Von Bedeutung ist die zunehmende Forcierung der aktiven Rolle der Hochschulen in nationalen und regionalen Innovationsprozessen und -strategien (,Smart Specialization“) sowie die Kommerzialisierung von Wissen durch die Hochschulen (,Third Mission“) z.B. durch Patente, Lizenzen oder SpinOffs. Initiativen auf Bundesebene, die sich Themen wie „Entrepreneurial University“, der Schaffung und Zugänglichkeit von Forschungsinfrastrukturen oder der Weiterentwicklung des Verwertungsmanagements an Hochschulen widmen, zielen einerseits auf internationale Wettbewerbsfähigkeit und andererseits auf einen stärkeren Beitrag zum unternehmerischen Innovationsgeschehen ab.

Gemeinsam mit einer „Spin-Off und Start-up Szene“ hat sich in Österreich mittlerweile ein vielfältiges Unterstützungsangebot gebildet, das von einer wachsenden Anzahl von Akteuren bereitgestellt wird:

- Ideenwettbewerbe, Auszeichnungen und Informationsveranstaltungen,

- Beratungs- und Coaching-Angebote,

- Inkubatoren und Co-working-Spaces sowie

- Förderungs- und Finanzierungsangebote.

Die Entwicklungen in der Gründungslandschaft werden von sehr unterschiedlichen Akteuren vorangetrieben. Selbst auf der Bundesebene im Fokus innovativer wachstumsorientierter Gründungen haben sich in relativ kurzer Zeit gleich mehrere Unterstützungsangebote - u.a. das AplusB-Programm, die Start-up-Initiative innerhalb der FFG-Basisprogramme, die Programme der aws - Austria Wirtschaftsservice - entwickelt, die sehr häufig auch parallel von einzelnen Gründungen in Anspruch genommen werden (siehe dazu auch Abschnitt 5.2.2).

In Berufung auf die Aussagen befragter Stakeholder auf der nationalen und regionalen Ebene hat das AplusB-Programm einen nachvollziehen Beitrag zur positiven Entwicklung der „Gründerszene“ in Österreich beigetragen.

Während die heimische Förderungslandschaft kontinuierlich verbessert wurde und private Initiativen ihr Angebot ausgebaut haben, bestehen nach Aussagen der InterviewpartnerInnen jedoch weiterhin Defizite, wo sich in den letzten Jahren nur wenig bis gar nichts verändert hat. Dies betrifft:

- Zentrale Rahmenbedingungen wie etwa eine mangelhafte Verfügbarkeit von Venture Capital für junge, innovative Unternehmen, vor allem in Frühphasen; eine unzureichende Stimulierung privater Risikokapitalbereitstellung;

- Schwächen in der Transformation von F\&E-Ergebnissen in die Wirtschaft;

- Eine Risiko-averse (Unternehmens-)Kultur sowie ein damit verbundenes, wenig ausgeprägtes Entrepreneurship-Denken;

Die Ausgründung von Spin-Offs ist aber für die Mutterorganisation und für die GründerInnen mit teils großen Herausforderungen verbunden. Ein zentraler Befund in den für diese Untersuchung geführten Interviews ist, dass der Hochschulbereich in den letzten Jahren Themen wie Entrepreneurship und Spin-offs mit erhöhter Sensibilität wahrnimmt, wobei das Engagement jedoch unterschiedlich intensiv und von verschiedenen Herangehensweisen, Schwerpunktlegungen und Ideologiediskussionen im Innovationssystem geprägt ist. Die Hochschulen haben in den vergangenen Jahren in sehr unterschiedlichem Ausmaß verschiedene Ansätze und Routinen entwickelt, um Gründungen und den damit einhergehenden Transfer von Wissen und geistigen Eigentumsrechten (IPR) zu unterstützen.

8 Vgl. BMWFW, BMVIT (2014): Österreichischer Forschungs- und Technologiebericht 2015. 
Auf Grund der zur Verfügung stehenden Ressourcen und Instrumente sind wissenschaftliche Einrichtungen nur eingeschränkt in der Lage, hoch-komplexe Gründungprojekte über einen längeren Zeitraum intensiv zu begleiten. Im Zusammenspiel mit AplusB können Hochschulen und Forschungseinrichtungen allerdings einen wesentlichen Beitrag im Bereich der Bewusstseinsbildung und Vorfeldarbeit übernehmen.

Private Inkubatoren sowie regionale Impulszentren, die üblicherweise mit gewerblichen Gründungen konfrontiert sind, sind wenig auf unerfahrene und dennoch selbstbewusste AkademikerInnen eingestellt, die neben der Infrastruktur vor allem eine intensive, beratende Begleitung benötigen.

Wie im Rahmen der geführten Gespräche deutlich wurde, können private Anbieter das Angebot von AplusB ergänzen, jedoch nicht im derzeitigen Umfang ersetzen oder übernehmen. Private Initiativen haben üblicherweise nicht das Ziel und sind nicht dafür ausgestattet, Gründungen zu stimulieren (z.B. Bewusstseinsbildung, Pre-Inkubation) oder risikoreiche und erheblich entwicklungsbedürftige Projekte in frühen Stadien zu übernehmen. Schließlich ist anzumerken, dass private Initiativen regional sehr ungleich verteilt sind.

\subsection{Ziele und Methodik der Evaluierung}

Ziel der vorliegenden Evaluierung ist die gesamtheitliche Betrachtung der erzielten Wirkungen des Programms Academia plus Business (AplusB) nach seiner nun mehr als zehnjährigen Laufzeit. Im Mittelpunkt der Untersuchungen stehen:

- das Konzept, die Umsetzung (Zielerreichung) und die bisherigen Wirkungen des Programms die hinsichtlich ihrer Effektivität und Effizienz beurteilt werden sollen,

- sowie die Struktur, das Umfeld und die Performance der AplusB-Zentren.

Die Untersuchung erfolgte auf Basis eines Multi-Ebenen-Ansatzes, welcher insbesondere zwischen der Programm-, Zentrums- und Gründerperspektive differenzierte, sowie einer Analyse aus mehreren Blickwinkeln, unter Einbindung relevanter, regionaler Stakeholder. Der Methodenmix, bestehend aus Literatur- und Dokumentenanalysen, die Erstellung eines Logic-Charts, leitfadengestützte Interviews, Sekundärdatenanalyse mit Kontrollgruppenvergleich, Fallstudien und Fokusgruppen, bieten eine gute Basis für die umfassende Identifikation und Diskussion der zentralen Wirkungen sowie für mögliche Herausforderungen für die künftige Gestaltung des Programms.

Die Evaluierung bezieht sich auf das Gesamtprogramm und nimmt keine Bewertung einzelner Zentren oder regionaler Stakeholder vor. Basierend auf den Analysen, Einschätzungen und Bewertungen der unterschiedlichen Betrachtungsebenen wurde eine Zusammenschau vorgenommen und mit den Zielsetzungen und der Mission von AplusB in Verbindung gesetzt. Darauf aufbauend wurden Handlungsoptionen und Perspektiven für eine Weiterentwicklung des Programms bzw. der Arbeit in den Zentren abgeleitet. 


\section{Programmgenese und -entwicklung}

\subsection{Das Programm im Überblick}

Das Academia-plus-Business-(AplusB)-Programm wurde 2001 durch das Bundesministerium für Verkehr, Innovation und Technologie (bmvit) zur Förderung von Ausgründungsaktivitäten aus Hochschulen und außeruniversitären Forschungseinrichtungen ins Leben gerufen. ${ }^{9}$ Als Zielgruppen werden Postgraduierte und promovierte WissenschafterInnen an Universitäten und Fachhochschulen sowie wissenschaftliche MitarbeiterInnen an außeruniversitären Forschungseinrichtungen angegeben. Mit der operativen Umsetzung und Abwicklung des Programms ist seit 2004 die Österreichische Forschungsförderungsgesellschaft (FFG) beauftragt. Zuvor wurde AplusB von der Technologie-ImpulseGesellschaft (TIG) abgewickelt.

Das Programm setzt eine Abstimmung zwischen Bund und Ländern voraus und sieht die Einrichtung von AplusB-Zentren (Inkubatoren) vor, die innovative und wachstumsorientierte Gründungen nicht nur im Vor- und Nachfeld der Inkubation (Verweildauer im Zentrum), sondern bereits in der Form von Maßnahmen der Sensibilisierung und Bewusstseinsbildung vor Ort unterstützen.

Der ursprüngliche Fokus des Programms liegt auf akademische GründerInnen im engeren Sinn, die forschungs- und technologieintensiv ausgerichtet sind und daher in besonderem Maße dazu beitragen können, die technologische Wettbewerbsfähigkeit zu verbessern.

Zudem stellt das Programm die wirtschaftliche Umsetzung von akademischem Know-how in den Vordergrund, welches einerseits in akademischen Einrichtungen und/oder andererseits von AkademikerInnen mit Erfahrungen aus der Wirtschaft entwickelt wurde und damit auf eine Intensivierung und Verfestigung der Forschungskooperationen zwischen Wissenschaft und Industrie abzielt.

Das Programm wird seit 2001 aufgrund von bisher drei Sonderrichtlinien ${ }^{10}$ durchgeführt. In der Anpassung von 2006 wurde die Bundesförderungsquote für die Jahre 6 bis 10 erhöht, 2011 wurde eine Erweiterung der Richtlinien (,AplusB 2.0“) mit einer eigenen Leistungsbeschreibung vollzogen. Aplus $B$ wird auf der Basis von Zentren, über welche die konkrete Unterstützung und Coaching potentieller GründerInnen erfolgt, in den jeweiligen Bundesländern umgesetzt. 2001 und 2004 fanden zwei offene Ausschreibungsrunden im Wettbewerbsmodus statt, auf deren Basis die Genehmigung von insgesamt neun ${ }^{11}$ AplusB-Zentren erfolgte.

Als Laufzeit der Förderung wurden maximal zehn Jahre fixiert. Ein Zentrum (v-start) ist 2007 aus dem Programm ausgeschieden. 2008 erfolgte eine erste, externe Zwischenevaluierung ${ }^{12}$ des Programms. Diese kam zu der wesentlichen Einschätzung, dass der ursprüngliche Plan, die Zentren nach zehn Jah-

9 Durch das AplusB-Programm sollen Zentren geschaffen werden, ,die WissenschaftlerInnen aus Universitäten, Fachhochschulen und Forschungseinrichtungen die Möglichkeit geben, sich durch Stimulierung, Qualifizierung und Unterstützung auf dem oft schwierigen Weg von einer guten Idee zu einem Unternehmen helfen und begleiten zu lassen. Dabei geht es nicht nur um konkrete Unterstützung im Gründungsprozess, sondern auch darum, Unternehmertum als Option im akademischen Denken und Handeln stärker zu verankern.“ (Sonderrichtlinie Juni 2001).

10 i) Sonderrichtlinien für die Förderung von Zentren zur Unterstützung akademischer GründerInnenAplusB Academia Business Spinoff Gründerprogramm 2001 bis 2006, Juni 2001; ii) Sonderrichtlinien für die Förderung von Zentren zur Unterstützung akademischer Gründerinnen AplusB Academia Business Spin-off Gründerprogramm 2001 bis 2006, September 2006; iii) Sonderrichtlinien für das Programm AplusB 2.0, Juli 2011.

11 Das sind: accent (Niederösterreich), BCCS-Business Creation Center Salzburg, build! (Kärnten), CAST (Tirol), INiTS (Wien), SPG-Science Park Graz (Steiermark), tech2b (Oberösterreich), v-start (Vorarlberg) und das ZAT - Zentrum für Angewandte Technologie (Steiermark). Letzteres wurde als Pilotprojekt zwischen den Ausschreibungsrunden zur Förderung genehmigt.

12 Vgl. Heydebreck, P.; Petersen K. (2008). AplusB Acadmia Business Spin-off Gründerprogramm - Zwischenevaluierung. 
ren Lauf- und Förderungszeitraum in die Selbständigkeit zu entlassen, vor dem Hintergrund der geltenden Richtlinien und Rahmenbedingungen nicht möglich ist. Der Fokus auf aussichtsreiche, aber dennoch anspruchsvolle Gründungsvorhaben mit erheblichem Entwicklungsbedarf, die von der Frühphase an intensiv begleitet werden, stellt gegenüber privat (re-)finanzbaren Aktivitäten ein Differenzierungsmerkmal dar und rechtfertig gleichzeitig den dauerhaften Einsatz öffentlicher Mittel.

Grundlage für die Weiterführung der Zentren sind Evaluierungen, die, wie im Konzept ${ }^{13}$ zur Evaluie- $^{-}$ rung der AplusB-Zentren vorgesehen, im dritten (3JE), fünften (5JE) sowie zehnten Jahr (10JE) des Bestehens anzusetzen sind. Während die 3JE primär Empfehlungs- und Lerncharakter hatte und auf die Untersuchung der Aufbau- und Managementarbeit sowie den bisherigen Zielerreichungsgrad abstellte, fokussierte die 5JE neben der Zentrumsarbeit stärker auf die Zahl und Qualität der Gründungsvorhaben. Konsequenz der 5JE war eine „Stop or Go“-Entscheidung für die Fortsetzung des Förderungsvertrags. Die 3JE wurde - aufgrund der unterschiedlichen Startdaten der Zentren - 2005 (5 Evaluierungen), 2006 (1) und 2008 (3) durchgeführt, die 5JE 2006 (1), 2007 (4), 2008 (1) und 2010 (2). Die lt. Konzept vorgesehenen ex-post Evaluierungen im zehnten Jahr wurden nicht durchgeführt. Allerdings wurde im Rahmen der Antragsbewerbung für die dritte Phase eine Bewertung der bisherigen Leistungen durch das AplusB-Bewertungsgremium durchgeführt. Die Grundlage bildet hier der Antrag mit einer Rückschau und einer Vorschau der AplusB-Zentren.

Der Empfehlung zur Fortführung des Programms folgend wurde 2012 ein zweites Ausschreibungsverfahren „AplusB 2.0“ für die nunmehr dritte Förderungsperiode (1. 2002/07; 2. 2007/12; 3. 2012/17) ${ }^{14}$ angesetzt. Der entsprechenden Sonderrichtlinie ist dabei zu entnehmen, dass der Fokus auf die Weiterführung bereits geförderter Strukturen gelegt und keine neuen Strukturen aufgebaut werden sollen.

- Fünf bestehende AplusB-Zentren wurden zur Einreichung eines Weiterführungsantrages eingeladen, wobei die Weiterförderung aller Zentren genehmigt wurde.

- 2013 wurden die übrigen drei AplusB-Zentren zur Einreichung eines Weiterführungsantrages eingeladen. Für zwei Zentren wurde eine Weiterförderung genehmigt, ein Ansuchen (BCCS) wurde abgelehnt.

Somit werden mit Beginn 2015 insgesamt sieben Zentren in sechs Bundesländern gefördert. Dabei verfügt die Steiermark über zwei Zentren (ZAT, SPG), während es in Vorarlberg, Burgenland und Salzburg kein gefördertes AplusB-Zentrum gibt. Die Umsetzung des AplusB-Programms wird durch ein umfassendes Berichtswesen und Monitoring begleitet.

- Zum Berichtswesen zählen zum einen Statusberichte, die halbjährlich anzufertigen sind, sowie Jahresberichte, die sich auf das gesamte Förderjahr beziehen. Beide Berichte geben einen Überblick über die abgelaufene Periode, darunter eine Beschreibung der Aktivitäten und Maßnahmen des Zentrums, betreute Gründungsvorhaben und Coaching-Projekte, eine Darstellung des NonAplusB-Bereichs sowie Erläuterungen zu Rechnungswesen und Controlling. Ab der dritten Förderungsperiode wurde das Berichtswesen auf Jahresberichte umgestellt.

- Ergänzend zum Berichtswesen wird seit Programmstart ein „Gründungs-Monitoring“ eingesetzt, welches an unterschiedlichen Zeitpunkten Daten von GründerInnen bzw. den gegründeten Unternehmen erhebt. Das Gründungs-Monitoring erfolgt bei der FFG.

13 Vgl. AplusB Academia Business Spin-off Gründerprogramm: Konzept zur Evaluierung der AplusB-Zentren. Wien, Februar 2002.

14 Es ist an dieser Stelle anzumerken, dass nicht alle Zentren zum selben Zeitpunkt im Programm gestartet sind. Ab der dritten Förderungsperiode wurden die Förderzeiträume und Anforderungen für alle Zentren gleichgesetzt. 
Seit 2005 finden regelmäßig Treffen der „AplusB-Plattform“ statt, eine gemeinsame Einrichtung, die dem Informations- und Kommunikationsaustausch zwischen den AplusB-Zentren, der FFG und dem bmvit dient.

Im Hinblick auf die Öffentlichkeitsarbeit sind der 2011 gegründete Verein „Österreichisches Inkubatorennetzwerk AplusB“, unter dessen Dach sich die AplusB-Zentren zusammengeschlossen haben, sowie der gemeinsame Webauftritt (www.AplusB.biz) zu nennen. In den einzelnen Regionen werden von den Zentren darüber hinaus unterschiedliche Kommunikationsinstrumente und Marketingmaßnahmen eingesetzt.

\subsection{Mission und Ziele}

Auf Basis der Dokumentenanalyse und konzeptionellen Vorarbeiten ${ }^{15}$ sowie der durchgeführten Interviews wurde die Programmlogik von AplusB mit Hilfe eines Logic Charts nachgezeichnet. Dieser stellt den Zusammenhang zwischen Mission, Zielen, Maßnahmen und Aktivitäten sowie den erwarteten Wirkungen des Programms auf unterschiedlichen Ebenen (Output, Outcome, Impacts) dar (siehe Abbildung 2). Insbesondere die Messgrößen zur Erhebung des Nutzens bzw. der Wirkungen des Programms wurden im Zuge von Eigenüberlegungen im Evaluierungsteam ergänzt und in den durchgeführten Interviews ausführlich diskutiert.

Die Mission des Programms ist es, einen Brückenschlag zwischen Wissenschaft (Academia) und Wirtschaft (Business) durch eine Verankerung und Entwicklung von Unternehmertum im akademischen Denken und Handeln zu schaffen. An anderer Stelle ${ }^{16}$ versteht sich das AplusB-Programm wie folgt: „Die AplusB-Zentren fungieren als Brückenbauerinnen zwischen Wissenschaft (Academia) und Wirtschaft (Business) mit dem Ziel, die Erfolgschancen hochinnovativer und technologieorientierter Startups aus den unterschiedlichsten Branchen signifikant zu erhöhen. “

$\mathrm{Zu}$ den Zielsetzungen des Programms zählen:

- Die Umsetzung aktueller Forschungsergebnisse in unternehmerische Innovation: Dies umfasst die in $\operatorname{der}$ AplusB Sonderrichtlinie AplusB ${ }^{17}$ angeführten Ziele i) einer Verbesserung der unternehmerischen Verwertung von Forschungsergebnissen sowie ii) der Unterstützung spezifischer (anderer) Maßnahmen des Technologietransfers.

- Die Unterstützung von WissenschafterInnen aus Universitäten, Fachhochschulen und außeruniversitären Forschungseinrichtungen bei der Umsetzung von Unternehmensideen: Dazu zählt die in der Sonderrichtlinie für das AplusB-Programm ${ }^{18}$ anvisierte iii) Erweiterung des Potentials an Unternehmensgründungen aus den genannten Einrichtungen sowie die in den letzten Jahren stärker ins Blickfeld rückende Unterstützung von Frauen als potentielle GründerInnen ${ }^{19}$.

- Eine Steigerung der Gründungsdynamik an Universitäten, Fachhochschulen und außeruniversitären Forschungseinrichtungen: Dies fasst die in der Sonderrichtlinie genannten Ziele eines iv) dau-

15 Vgl. Zinöcker, K. (2003): Die Implementierung von Evaluierungssystemen in FTE-Programmen. Konzeptionelle und operationale Überlegungen an Hand des österreichischen Spin-Off Programms AplusB. InTeReg-Working paper 11-2003; TIG (2002): Konzept zur Evaluierung der AplusB-Zentren.

16 Siehe dazu: http://www.AplusB.biz/was-ist-AplusB.html

17 Vgl. Sonderrichtlinien für die Förderung von Zentren zur Unterstützung akademischer GründerInnen AplusB Academia Business Spin-off Gründerprogramm 2001 bis 2006 (Vers. September 2006).

18 Als Ergänzung zur Sonderrichtlinien wurde das Leistungsprofil erstellt. Das Leitungsprofil hat Referenzcharakter für die Zentren in Bezug auf die Umsetzung im förderbaren Rahmen.

19 Vgl. „Erhöhung Potential Gründerinnen“; in: AplusB 2.0. Das neue Leistungsprofil der AplusB-Zentren. Wien, Jänner 2011. 
erhaften Anstiegs der Anzahl akademischer Spin-offs sowie v) die Steigerung der Qualität (Technologie- und Wissensintensität) und Erfolgswahrscheinlichkeit dieser Gründungen zusammen.

Im Zuge des Programms werden seit Januar 2015 in sieben AplusB-Zentren akademische Unternehmensgründungen und Spin-offs in Form eines „One-Stop-Shops“ gefördert und unterstützt. Anhand des gezeichneten Logic Charts lässt sich der Maßnahmenmix grob in zwei Bereiche unterteilen: Eine allgemeine „Zentrums- bzw. Öffentlichkeitsarbeit“ steht dabei einer konkreten Auseinandersetzung mit und Unterstützung von (potentiellen) GründerInnen gegenüber. Letzteres unterscheidet 1t. aktueller Sonderrichtlinie „AplusB 2.0“ die Bereiche „Awareness und Coaching“ (Pre-Inkubation), „Gründungsbetreuung“ (Inkubation) sowie „Alumni-Betreuung“ (Post-Inkubation). Eine detaillierte Diskussion dieser Bereiche findet sich in Abschnitt 3.

$\mathrm{Zu}$ den direkten Output-Größen des Programms ${ }^{20}$ sind neben der Anzahl der unterschiedlichen Maßnahmen und Stimulierungsaktivitäten vor allem die Anzahl der Gründungsvorhaben bzw. die tatsächlichen Gründungen zu zählen. Ein insbesondere für die vorliegende Evaluierung positiver Effekt ist das Monitoring der GründerInnen bzw. das Berichtswesen, welches die Analysen der unterschiedlichen Indikatoren unterstützt. Mittelbare Effekte umfassen die (Veränderungen in der) Wahrnehmung sowie Folgewirkungen der Maßnahmen und Aktivitäten der Zentren. Die langfristigen Effekte stellen die finalen, mitunter schwierig zu beobachtenden und differenzierbaren Wirkungen des Programms dar, die insbesondere vor dem Hintergrund der gesetzten Ziele zu bewerten sind.

20 Siehe auch: Zinöcker, K. (2003): Die Implementierung von Evaluierungssystemen in FTE-Programmen. Konzeptionelle und operationale Überlegungen an Hand des österreichischen Spin-Off Programms AplusB. InTeReg-Working paper 11-2003; TIG (2002): Konzept zur Evaluierung der AplusB-Zentren. 


\section{Abbildung 2 Logic Chart des AplusB-Programms}

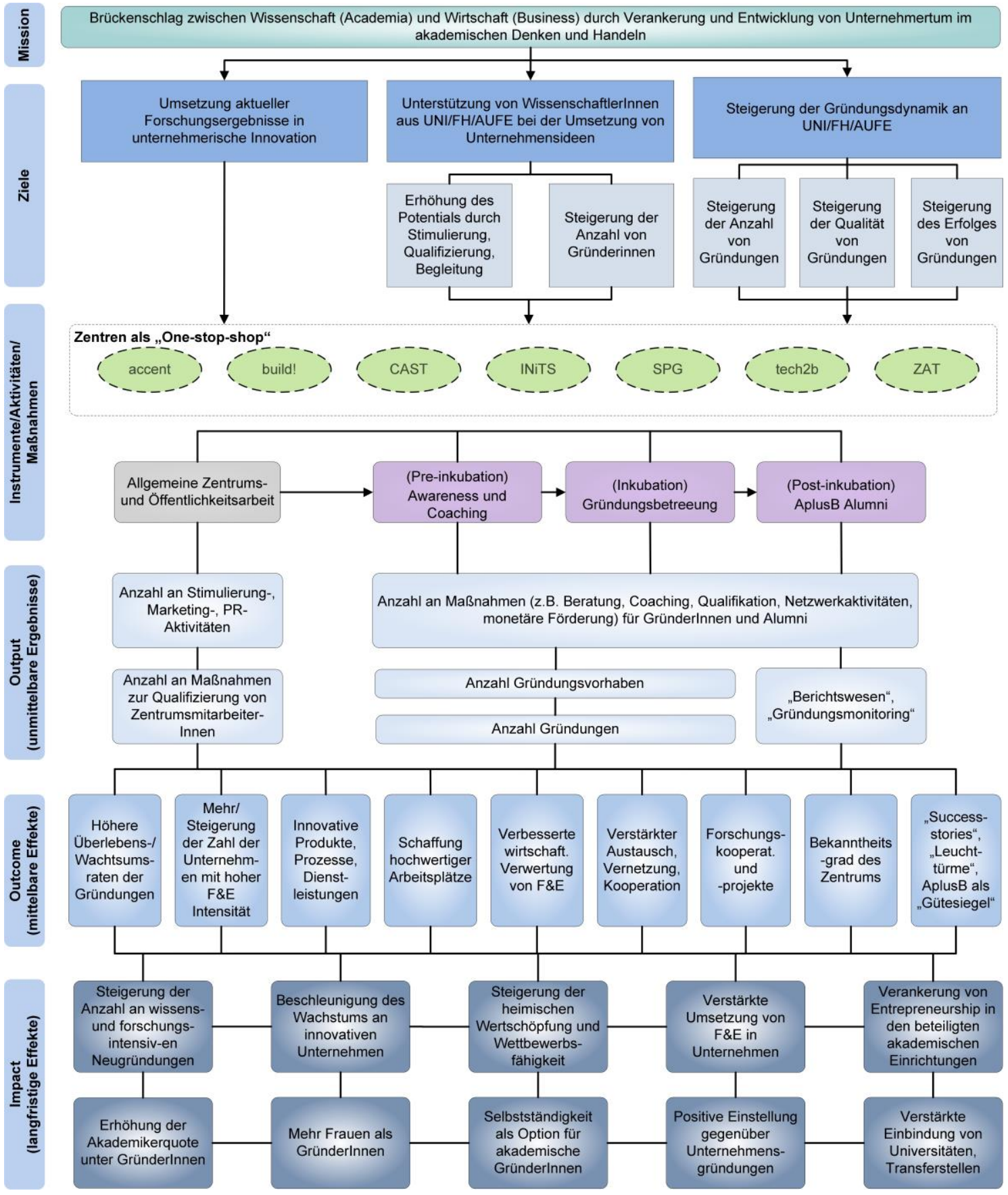

Quelle: Darstellung JR

Lt. Sonderrichtlinie „AplusB 2.0“ wurden die Schwerpunkte für die laufende 3. Förderungsperiode (2012/17) wie folgt definiert:

- Sichtbarkeit und Intensivierung der Förderung der Pre-Inkubation Phase zur Steigerung der Qualität der Projekte vor Aufnahme;

- Strukturelle Neuerung durch die Festlegung einer Mindestgröße für den Betrieb eines Zentrums, damit die Qualität der Gründungsbetreuung über alle Zentren hinweg gewährleistet ist;

- IPR-Betreuung verstärken zur Klärung der Verwertungsrechte vor der Aufnahme ins Zentrum und Sicherung dieser; 
- Alumni-Betreuung in erster Linie durch Beratungsleistungen, die auch nach dem Ausscheiden aus dem AplusB-Zentrum zu einem späteren Zeitpunkt konsumiert werden können;

- Erweiterung der bisherigen Zielgruppe um die Gruppe der AkademikerInnen mit mehrjähriger Berufserfahrung

\subsection{Bewertung von Zielsetzung und Konzeption}

Nachfolgend werden die für die Evaluierung formulierten Fragen beantwortet.

- Welche übergreifenden und spezifischen Ziele verfolgt das Programm? Welche Interessen stehen dahinter? Wo liegen die Prioritäten? Wo liegen etwaige Zielkonflikte? Sind diese Zielsetzungen konsistent bzw. (empirisch) überprüfbar?

AplusB ist in seinem Ursprung ein zielgruppenspezifisches Programm zur Unterstützung und Förderung von Ausgründungsaktivitäten aus Hochschulen und außeruniversitären Forschungseinrichtungen, als ein Kanal zur kommerziellen Nutzung von wissenschaftlichen Ergebnissen und/oder Kompetenzen. $\mathrm{Zu}$ den Zielsetzungen des Programms zählen die Umsetzung aktueller Forschungsergebnisse in unternehmerische Innovation, die Unterstützung von WissenschafterInnen aus Universitäten, Fachhochschulen und außeruniversitären Forschungseinrichtungen bei der Umsetzung von Unternehmensideen und eine Steigerung der Gründungsdynamik an Universitäten, Fachhochschulen und außeruniversitären Forschungseinrichtungen. AplusB setzt bereits vor der eigentlichen Unternehmensgründung an, indem versucht wird, ForscherInnen und WissenschafterInnen die Selbstständigkeit als möglicher Karriereschritt nahe zu bringen. Dadurch sollen neue, innovative Unternehmen mit hochwertigen Arbeitsplätzen entstehen und ein positives Klima für Unternehmensgründungen entwickelt werden.

Die verschiedenen Teilziele des Programms (z.B. dauerhafter Anstieg der Anzahl akademischer Spinoffs, Verbesserung der unternehmerischen Verwertung von Forschungsergebnissen) wurden beim Übergang in die 3. Förderungsperiode (,AplusB 2.0“) ohne Anpassung übernommen. Der Grund hierfür wird in der nach wie vor aktuellen Zielsetzung des Programms, insbesondere vor dem Hintergrund der 2011 formulierten FTI-Strategie, welche auf eine Steigerung der Anzahl an wissens- und forschungsintensiven Neugründungen abzielt, gesehen.

- Spezifische Entwicklungspfade, wie etwa die in den letzten Jahren für akademische Spin-Offs wichtiger gewordenen Industriekooperationen, finden sich im Programm weder auf Ebene der Ziele noch auf Ebene der Unterstützungsmaßnahmen. Eine entsprechende Anpassung ist nach Konsultation mit den einzelnen Zentren zu prüfen.

- Die nach der ersten Förderungsperiode aufgenommene Erweiterung Ausweitung auf GründerInnen mit akademischen Hintergrund die aus der Privatwirtschaft (nicht unmittelbar aus der Forschung oder Karriere an einer öffentlichen Forschungseinrichtung) kommen, spiegelt sich im gegenwärtigen Zielsystem nur bedingt wider und bedarf einer klaren Deklaration.

- Im Gegensatz dazu wurde eine Erweiterung und Vervollständigung des künftig umzusetzenden Leistungspakets vorgenommen und in einen modularen Ansatz mit MUSS- und KANNBausteinen eingebettet. Dem Aufnahmeprozess ins Zentrum (Pre-Inkubation) wurde dabei mehr Gewicht im Leistungsspektrum eingeräumt, die Nachbetreuung ausgeschiedener Gründungsprojekte (Post-Inkubation) wurde überhaupt neu festgeschrieben.

Grundlegende Zielkonflikte im Programmgefüge sind nicht erkennbar, was vorwiegend auf die nunmehr fast 13-jährige Lebenszeit des Programms sowie die damit verbundenen, laufenden Anpassungen und Korrekturen zurückzuführen ist. 
Der Übergang in die 3. Förderungsperiode hat, wie erwähnt, zu einer Verbreiterung des Interventionsmusters, insbesondere vor dem Hintergrund der Erweiterung der Betreuungsphasen sowie einer Ausweitung der Zielgruppen, geführt.

Die Erweiterung des Programmspektrums hat indes zu keiner Konkretisierung von bestehenden bzw. zur Formulierung neuer Zielgrößen geführt. Als Beispiel ist etwa der Punkt „Erhöhung Potential GründerInnen“ zu nennen, welcher in „AplusB 2.0“ zwar explizit aufscheint, jedoch ohne Messgröße ausgestattet wird. Das Berichtswesen bzw. das Gründungs-Monitoring sind hier entsprechend anzupassen, um die Messbarmachung und Vergleichbarkeit der Performance für künftige Analysen zu unterstützen.

Die Vorfeldarbeit und „Pre-Inkubation“ grenzt sich nicht ganz eindeutig von der „,allgemeinen Zentrums- und Öffentlichkeitsarbeit“ ab, wie der Logic Chart auch andeutet und am Beispiel der „Bewusstseinsbildung in der Gesellschaft und bei den Stakeholdern" deutlich wird. Wie auch in den durchgeführten Einzelgesprächen sowie Fokusgruppen (mit regionalen Fördergebern, Trägern und Zentrenleitungen) verdeutlicht, rechtfertigt sich eine separate Betrachtung der Awareness-Phasen aufgrund der regional sehr unterschiedlichen Voraussetzungen und damit einhergehend Herangehensweisen.

- Stimmen die expliziten und impliziten Ziele mit der Mission überein? Wie müssen Mission und Zielsetzungen zukünftig aufeinander abgestimmt werden, um den Nutzen bzw. die Wirkung zu verbessern?

Das im Zuge von „AplusB 2.0“ erweiterte Leistungsspektrum bildet sich aktuell nicht vollständig in der Programmmission ab. Die Formulierung der Mission ist aufgrund unterschiedlicher Definitionen zu schärfen bzw. abschließend zu diskutieren, um auch eine bessere Abgrenzung zu den spezifischen Zielsetzungen zu schaffen. Die Ergebnisse der Dokumentenanalyse sowie der geführten Interviews erwecken den Eindruck, AplusB bewege sich in der Kohärenz der Zeile zueinander vom eigentlichen Spin-Off Fokus weg, hin zu einem etwas weiter gefassten Fokus auf innovative wachstumsorientierte Start-ups. Diese Erweiterung ist nachvollziehbar und reagiert unter anderem auf ein sich wandelndes Bild von Karrieremobilität, von lebenslangem Lernen sowie dem Interagieren von Wirtschaft und Wissenschaft. Aus heutiger Perspektive könnte allerdings - unter Berücksichtigung der künftigen Ausrichtung der Zentren - die Erarbeitung eines dahingehend geschärften Zielsystems die Abstimmung im Interventionsgefüge sowie auch die Ergebnisbewertung begünstigen. Dabei wäre zu diskutieren, was eine Hierarchie für die Maßnahmen und Aktivitäten bzw. überhaupt den Fokus des Programms bedeuten könnte.

- Ist die Konsistenz zwischen den Zielen, Aufgaben, Aktivitäten/Instrumenten/Prozessen und den (angestrebten) Ergebnissen konzeptionell gegeben?

Mit Hilfe der Logic-Chart-Analyse wurde ein erster Schritt zur Darlegung der Interventionslogik und zur Abstimmung zwischen Mission, Zielen, Maßnahmen und Aktivitäten sowie den erwarteten Wirkungen des Programms auf unterschiedlichen Ebenen (Output, Outcome, Impacts) vorgenommen. Das Interventionsmuster erscheint, trotz einer gewissen Verbreiterung im „AplusB 2.0“-Ansatz, als durchaus konsistent.

Sowohl auf der Ziel- als auch der Wirkungsebene bedarf es jedoch einiger Konkretisierungen. So wird nicht deutlich, ob sich eine ,dauerhafte Steigerung der Anzahl von Gründungen“ auf eine laufende Steigerung der Gründungszahlen oder die Nachhaltigkeit (Überleben) von Gründungen bezieht.

\section{- War die Wahl der Instrumente mit den Zielen kohärent?}

Die auf Zentrumsebene angebotenen Aktivitäten und Maßnahmen sind der Mission sowie den Zielsetzungen des Programms angemessen. Bei der Wahl einzelner Instrumente sind die regionalen Voraussetzungen sowie Schwerpunktlegungen der Zentren zu berücksichtigen. Da das Programm es im We- 
sentlichen den Zentren überlässt, welchen Ansatz sie wählen, bedeutet dies jedoch auch, dass das Leistungsverständnis und -angebot der AplusB-Zentren sich teils deutlich voneinander unterschieden. Zum anderen sind bei der Ressourcenaufteilung in der Beratung und Begleitung zwischen Pre-Inkubation, Projektbetreuung (Gründungsbegleitung) und Post-Inkubation teils große Unterschiede festzustellen.

- Sind Programmkonzeption und Instrumente unter den geänderten Rahmenbedingungen immer noch adäquat?

Universitäten und Forschungseinrichtungen nehmen Themen wie Entrepreneurship und Spin-offs mit erhöhter Sensibilität wahr. Leistungsvereinbarungen und Wissensbilanzen verlangen vom Hochschulsektor eine verstärkte Auseinandersetzung mit industrienaher Forschung bzw. Know-how-Transfer, wie etwa die Aufnahme von Spin-offs in die Performance-Indikatorik von Universitäten zeigt. Das Engagement in diesem Bereich ist jedoch, wie den Interviews zu entnehmen ist, unterschiedlich intensiv und von verschiedenen Herangehensweisen, Schwerpunktlegungen und Ideologiediskussionen (etwa: Rolle der Universitäten) im Innovationssystem geprägt.

Vor diesem Hintergrund bietet AplusB als „One-Stop-Shop“ seinen Trägern teils maßgeschneiderte Angebote, die von der Teilnahme bzw. Mitarbeit an Lehrveranstaltungen an der Hochschule bis hin zu spezialisierten Trainings, etwa im Bereich Schutz- und Eigentumsrechte (IPR), reichen können. Je nachdem, wie mit den Themen Entrepreneurship und Ausgründungen an den Trägereinrichtungen selbst umgegangen wird, können diese selektiv auf das Zentrumsangebot zugreifen. Aus Zentrumssicht kann man flexibel auf die Bedürfnisse der unterschiedlichen Trägereinrichtungen eingehen und rasch das entsprechende Angebot anpassen.

Aus dieser Perspektive ist die Programmkonzeption, mit der Ausnahme einiger noch ausstehender Präzisierungen und Schärfungen, sowie das flexible, bedarfsorientierte Angebot an Instrumenten nach wie vor als adäquat anzusehen. Sowohl in den Interviews, die mit Stakeholdern und GründerInnen geführt wurden, als auch in den Fokusgruppen wird die Zeitmäßigkeit und der Bedarf für ein AplusBProgramm hervorgehoben und auf eine strategische Lücke hingewiesen, die ohne das Programm offen bliebe. AplusB und die Zentren versuchen entsprechend der Ausgangskonzeption möglichst flexibel auf (regionale) Bedarfe zu reagieren. Die Stakeholder haben die Entwicklungen der jüngeren Zeit begleitet durch intensive Kommunikation der Zentren mitgetragen, wie auch die Interviews und Fokusgruppen mit Förderungsstellen und Trägerorganisationen bestätigt haben.

Es stellt sich aus der Sicht der EvaluatorInnen dennoch die Frage, ob das gegenwärtig verbreiternde Interventionsmuster mittelfristig Gefahr läuft, die originären Zielgruppen ein wenig aus den Augen zu verlieren.

- Sind die von Programmseite vorgesehenen Stimulierungsaktivitäten und Maßnahmen zur Unterstützung von forschungs- und technologieintensiven GründerInnen adäquat?

Die 1t. Sonderrichtlinie vorgesehenen Maßnahmen (z.B. Ideenwettbewerbe, Businessplanwettbewerbe, Lehrveranstaltungen und Seminare, Karriere und Berufsinformationsmessen, JungunternehmerInnenbzw. GründerInnentage, Sprechtage an den Forschungseinrichtungen, etc.) erscheinen aus heutiger Sicht - und vor allem aus Perspektive der einzelnen Zentren - als adäquat.

- Im Besonderen in der Stimulierungs- und Präinkubationsphase haben die einzelnen Zentren durchaus auch in Anknüpfung an regionale Gegebenheiten unterschiedliche Ansätze gewählt, die über die gemeinsame Plattform auch kommuniziert werden. Gegenwärtig erfolgt allerdings keine verbindliche Überlegung von „Good Practices“ in allen Zentren, was im Interesse einer effektiven „Vorarbeit“, aber auch der Ausschöpfung möglicher Kosteneinsparungspotentiale von Interesse sein kann. Dies gilt auch für den Umgang mit der Alumni-Betreuung, die in der jüngeren Zeit an Bedeutung gewonnen hat. 
Es wird in weiterer Folge jedoch zu klären sein, in welcher Form Stimulierungsmaßnahmen vorrangig in der Verantwortung der Zentren sein sollen und können oder Träger (konkret die öffentlichen Forschungseinrichtungen) in Zusammenarbeit mit der regionalen Wirtschaftsförderung hier noch stärker eingebunden werden können.

- Die Unterstützung der Gründungen baut im Wesentlichen auf Beratung, Coaching, Vernetzung und Qualifizierung auf. Darüber hinaus ist es zulässig und von nahezu allen Zentren geübte Praxis, Förderungen in sehr unterschiedlichem Ausmaß (die über die AplusB-Zentren abgewickelt werden) an Gründungen zu vergeben.

Dabei sind flexibel verwendbare Förderungsmittel von einer zweckgebundenen Kostenabdeckung für externe Beratungs- und Qualifizierungsangebote zu differenzieren. Es ist unbestritten, dass sich die Bedingungen für eine Bankenfinanzierung von Unternehmensgründungen in den letzten Jahren eher verschlechtert haben. Ungeachtet dessen besteht hier Koordinierungsbedarf mit komplementären nationalen und regionalen Förderangeboten in der Frühphase. 


\section{Programmumsetzung durch die Zentren}

Der folgende Abschnitt ist der Programmimplementierung gewidmet. Nach einer eingehenden Darstellung der finanziellen Rahmenbedingungen sowie der Mittelverwendung erfolgt an dieser Stelle eine Darstellung der Struktur bzw. Leistungen der Zentren. Eine Evaluierung der einzelnen Zentren erfolgte durch ein ausgewähltes Gremium jeweils nach drei bzw. fünf Jahren, allerdings nicht im Rahmen dieser Untersuchung. Ein weiterer Punkt gilt der Frage, inwieweit sich das AplusB-Programm im internationalen Umfeld auf Augenhöhe bewegen kann.

\subsection{Finanzierung und Mittelverwendung}

In den Förderungsperioden 1 (Mitte 2002 bis Mitte 2007) sowie 2 (Mitte 2007 bis Mitte 2012) belief sich das Budget von AplusB auf insgesamt rd. € 84,2 Mio. Für die Förderungsperiode 3 (Mitte 2012 bis Mitte 2017) wurde ein Planwert von rd. $€ 49,5$ Mio. angesetzt. Zusätzlich zur Bundesförderung war zur Finanzierung der AplusB-Zentren in der ersten Förderungsperiode zwingend eine Landesförderung erforderlich. In der zweiten und dritten Förderungsperiode konnte die Landesförderung auch durch alternative Finanzierungsquellen substituiert werden. Gegen Ende einer jeden Förderungsperiode wird auf der Basis von Zwischenevaluierungen über die weitere Förderwürdigkeit der Aktivitäten der Zentren befunden (,Stop-or-Go“ Entscheidung).

Hinsichtlich Budgetstruktur war bzw. ist folgender Schlüssel vorgesehen:

- 1. Förderungsperiode: Bundesanteil max. $45 \%$ der förderbaren Gesamtkosten bei einer Landesförderung von mind. $35 \%$. Der Eigenmittelanteil muss mind. $20 \%$ betragen.

- 2. Förderungsperiode: Bundesanteil max. $35 \%$ der förderbaren Gesamtkosten. Gefordert wird eine Landesförderung von in der Regel $30 \%$, mind. jedoch $25 \%$. Steigt der Eigenmittelanteil über $30 \%$, sinken der Landes- und der Bundesförderungsanteil aliquot im gleichen Ausmaß.

- 3. Förderungsperiode: Bundesanteil max. 30 \% der förderbaren Gesamtkosten (ohne KANNModule im Leistungsspektrum), max. $35 \%$ (mind. ein KANN-Modul). Die Eigenmittel des Zentrums müssen mind. $15 \%$ betragen, die Restfinanzierung kann über Landesmittel und regionale Förderungen erfolgen.

Diese Verteilung spiegelt sich in der tatsächlichen Budgetstruktur des Programms wider. In der ersten Förderungsperiode entstammten rd. $41 \%$ der Gesamtförderungen aus Bundesmitteln, rd. ein Drittel aus Landesmitteln und ein Viertel aus Eigenmitteln. 
Tabelle $1 \quad$ Finanzierungsstruktur nach Mittelherkunft, Förderungsperiode 1, 2 (Plan/Ist) und 3 (Plan)

\begin{tabular}{|c|c|c|c|c|c|c|c|c|c|c|}
\hline & \multicolumn{4}{|c|}{ 1. Förderungsperiode $2002 / 07 *$} & \multicolumn{4}{|c|}{ 2. Förderungsperiode $2007 / 12 * *$} & \multicolumn{2}{|c|}{$\begin{array}{l}\text { 3. Förderp. } \\
2012 / 17 * * *\end{array}$} \\
\hline & Plan & $\%$ & Ist & $\%$ & Plan & $\%$ & Ist & $\%$ & Plan & $\%$ \\
\hline Bund & 18.506 .010 & 42 & 17.635 .930 & 41 & 14.640 .642 & 32 & 13.166 .250 & 32 & 16.908 .300 & 34 \\
\hline Land & 15.391 .020 & 35 & 14.613.261 & 34 & 13.560 .275 & 30 & 12.081 .203 & 29 & 13.992 .573 & 28 \\
\hline $\begin{array}{l}\text { Eigen- } \\
\text { mittel }\end{array}$ & 10.544 .556 & 24 & 10.803 .926 & 25 & 17.090 .870 & 38 & 15.868 .588 & 39 & 18.551 .578 & 38 \\
\hline Summe & 44.441 .586 & 100 & 43.053 .117 & 100 & 45.291 .787 & 100 & 41.116 .041 & 100 & 49.452 .451 & 100 \\
\hline
\end{tabular}

Anmerkung: * 9;**8;**7 Zentren; 3. Förderungsperiode mit Planwerten. Rundungsdifferenzen nicht ausgeglichen. Quelle: Berichtswesen AplusB, Berechnung und Darstellung JR

In der zweiten Förderungsperiode ändert sich dieses Bild gemäß den Vorgaben: So wurden im Schnitt $40 \%$ der Gesamtförderung aus Eigenmitteln finanziert. Die Bundesmittel beliefen sich auf rd. $32 \%$, die Landesmittel auf rd. $29 \%$. Die Planzahlen für die aktuell laufende dritte Förderungsperiode orientieren sich an der Verteilung der zweiten Periode: $34 \%$ des Budgets sollen demnach aus Bundesmitteln, $28 \%$ aus Mitteln der Länder sowie $38 \%$ aus Eigenmitteln finanziert werden.

Auf Ebene der einzelnen Zentren sind leichte Schwankungen bei den Anteilen im Zeitverlauf festzustellen. In der ersten Förderungsperiode variiert der Anteil der Bundesmittel an der Gesamtförderung zw. $36 \%$ und $45 \%$. Das Zentrum build! finanzierte sich mit einem Drittel (32\%) aus Eigenmitteln, was den höchsten Wert in diesem Zeitraum darstellt, und $36 \%$ aus Bundesmitteln. 
Abbildung 3 Finanzierungsstruktur der AplusB-Zentren nach Finanzierungsquellen (in \%)

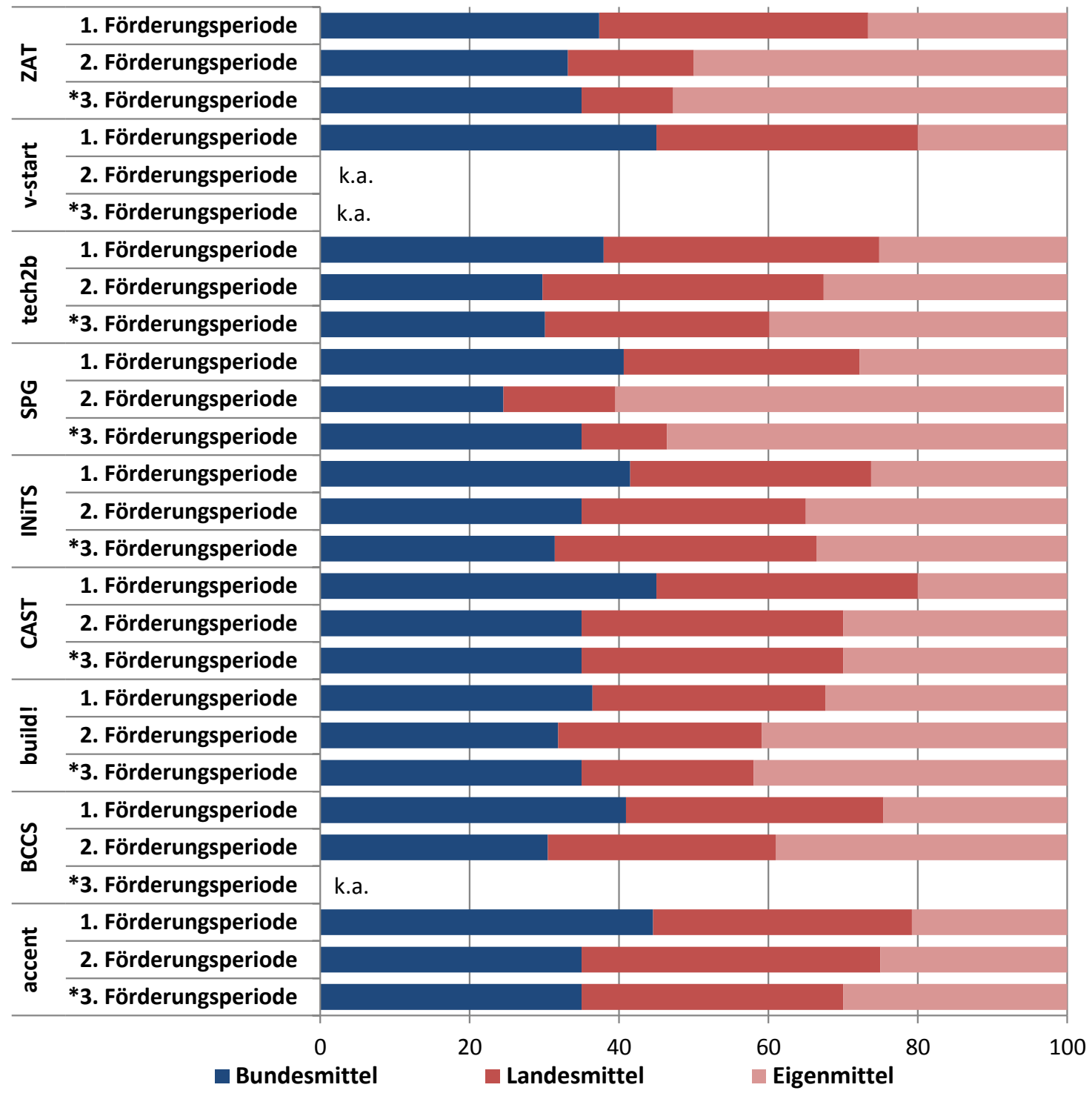

* 3. Förderungsperiode mit Planwerten. Quelle: FFG, JR-Berechnungen

Die Zentren accent, CAST und v-start finanzierten jeweils $20 \%$ des Gesamtbudgets aus eigenen Mitteln und $45 \%$ aus Bundesmitteln. In der zweiten Förderungsperiode liegt der Bundesanteil am Gesamtbudget der Zentren zw. $30 \%$ und $35 \%$. Der SPG finanziert sich zu $60 \%$ aus Eigenmitteln, die Zentren ZAT (50\%) und build! (41\%) erreichen ebenfalls einen hohen Eigenmittelanteil.

Die höchste Landesmittelfinanzierung entfällt mit $40 \%$ auf accent, der SPG und das ZAT erhielten mit $15 \%$ bzw. $17 \%$ am wenigsten an Landesmitteln, der Bundesmittelanteil in der dritten Förderungsperiode lag ebenfalls zwischen $30 \%$ und $35 \%$. Zu den Zentren mit dem höchsten Anteil an geplanter Eigenmittelfinanzierung zählen der SPG (54\%) und das ZAT (53\%), welche im Gegenzug über die wenigsten Landesmittel verfügen (11\% bzw. $12 \%$ ).

Im Zeitverlauf ergibt sich der Befund, dass der Anteil der Eigenmittel an den Zentren gestiegen ist (Ist 2002 - Plan 2017: +13\%-Punkte), was gleichzeitig eine Reduktion der Bundes- (-7 \%-Punkte) sowie Landesmittel (-6\%-Punkte) mit sich brachte. 
Der Finanzierungs- und Kostenplan für die dritte Förderungsperiode gibt einen Überblick über den geplanten Mitteleinsatz der Zentren nach Modulen (siehe Abbildung 4). Bei der Interpretation der Verteilung sind die regionalen Gegebenheiten und die damit einhergehenden unterschiedlichen Herangehensweisen und Schwerpunktsetzungen der Zentren zu beachten. Ein Vergleich mit Vorperioden ist nur beschränkt möglich, da insbesondere in den ersten Jahren Augenmerk auf Aufbau- und Entwicklungsarbeiten der Zentren gelegt wurde und sich das Zentrumsangebot dementsprechend daran ausrichtete.

Wie die Planwerte zeigen, entfällt der überwiegende Anteil der Kosten mit rd. $57 \%$ auf den Bereich der Inkubation bzw. Gründungsbetreuung, gefolgt von Bewusstseinsbildung (16,2\%), Pre-Inkubation und KANN-Modulen (jeweils ca. $9 \%$ ). Das ZAT (69 \%) und das tech2b (66,3\%) planen zwei Drittel der Aufwendungen im Bereich der Inkubation, bei INiTS ist es die Hälfte, bei CAST lediglich rd. 39 \%. Das Modul „Bewusstseinsbildung“ weist im Vergleich die größten Unterschiede auf: Während bei CAST hier fast ein Drittel (31\%) der Gesamtkosten anfallen sollen, sind es beim ZAT rd. $9 \%$ und beim accent $7 \%$.

\section{Abbildung 4 Gesamtkosten nach Modulen (in \%), 3. Förderungsperiode zwischen 2012-2017}

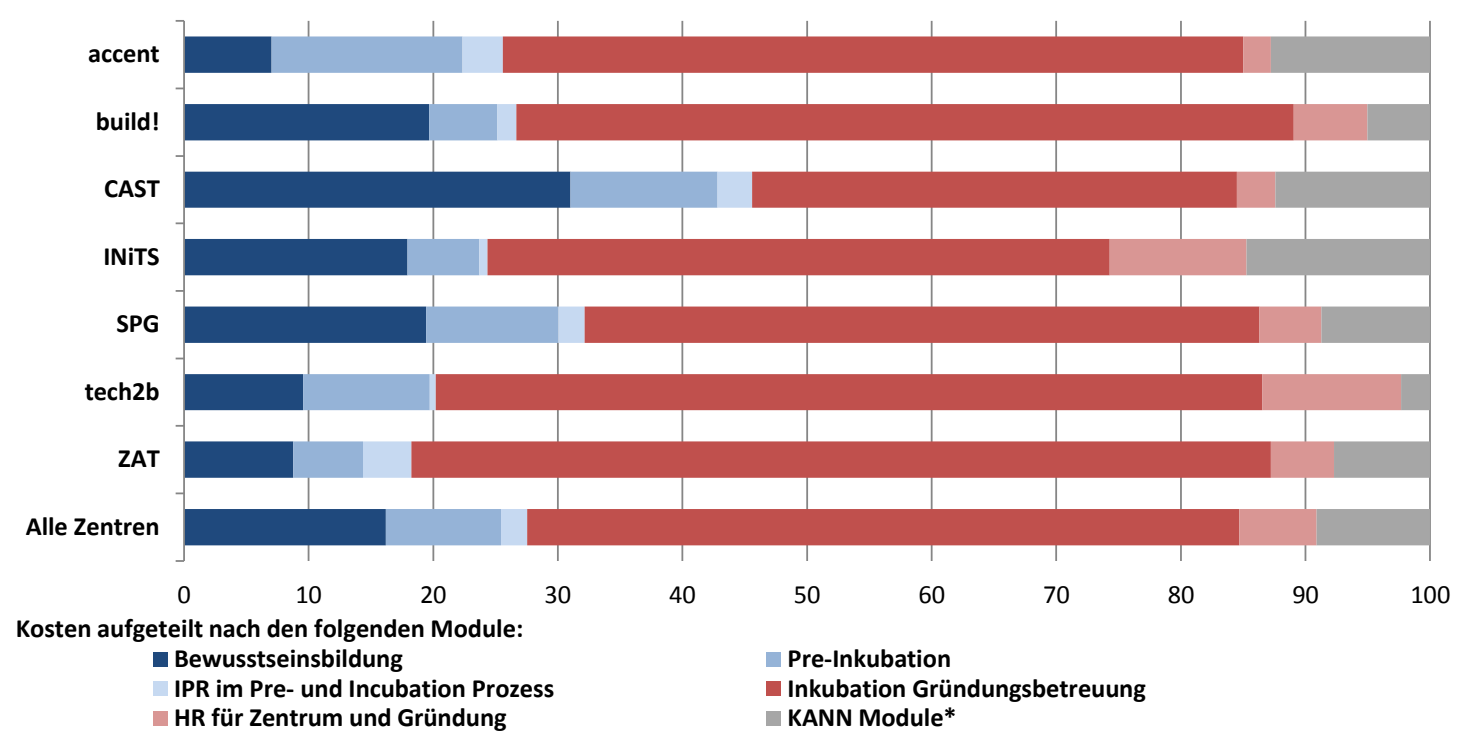

* KANN-Module: Alumni Betreuung, Finanzierung, Internationalisierung, IPR Services; Quelle: FFG, JR-Berechnungen

Im Rahmen der durchgeführten Rechnungshofberichte wurden unter anderem die Kosten je betreuter Gründung untersucht. Dies hat zu einer durchaus kontroversen Diskussion um die Bewertung unterschiedlicher Leistungsaspekte geführt. Des Weiteren wurden dadurch, wie aus den Gesprächen mit einzelnen Stakeholdern hervorging, die Identifikation etwaiger Einsparungspotentiale angeregt. Eine qualitative Bewertung der Arbeit der Zentren ist nicht Gegenstand der Programmevaluierung und soll den im Einzelnen durchgeführten Evaluierungen der AplusB-Zentren (sowie etwaigen Prüfungen durch den Rechnungshof) vorbehalten werden.

Aus der Sicht der Programmevaluierung ist dennoch ein Zusammenhang aufzugreifen, der sonst allzu leicht übersehen wird und mit der Ausrichtung des Programms und jenen den Zentren mitgegebenen Zielen zusammenhängt. Die Frage, die sich stellt, ist, welcher Aufwand tatsächlich einer einzelnen Gründung zugeschrieben werden kann. So nehmen die Zentren mittlerweile beachtliche und wachsende Aufgaben im Bereich der Sensibilisierung oder auch der Alumni-Betreuung wahr, die nicht direkt einem Inkubationsfall innerhalb der Betreuungsphase von 18 Monaten zugeordnet werden dürfen. 
Ein weiterer schwer abzugrenzender Punkt betrifft Förderungsmittel, die die Gründung im Zusammenhang mit der Inkubation zur Verfügung gestellt bekommt. Werden diese in der Berechnung berücksichtigt, so müssten sämtliche komplementäre Förderungen von Bundesstellen (aws, FFG) und Landesstellen einbezogen werden, die AplusB-GründerInnen einwerben.

Die folgende Tabelle 2 unternimmt anhand der Planzahlen für die dritte Förderungsperiode einen Versuch, einen relevanten Teil der Gesamtkosten (direkt/indirekt) einer inkubierten Gründung zuzuordnen. $^{21}$

Tabelle $2 \quad$ Verteilung und Zuordnung der Plankosten zur Inkubation nach Modulen

\begin{tabular}{|l|r|r|r|r|}
\hline Modul & $\begin{array}{l}\text { Anteil der } \\
\text { Module ä } \\
\text { den Gesamt-el der } \\
\text { ungschlüss } \\
\text { kosten } \\
\text { Module auf } \\
\text { Gründung }\end{array}$ & $\begin{array}{l}\text { Kosten } \\
\text { Durchschnitt je } \\
\text { Gründung }\end{array}$ & $\begin{array}{l}\text { Kosten min. } \\
\text { je Gründung }\end{array}$ \\
\hline Bewusstseinsbildung & $17 \%$ & $6 \%$ & $2.516,74$ & $2.400,67$ \\
\hline Pre-Incubation & $9 \%$ & $29 \%$ & $6.527,40$ & $3.634,76$ \\
\hline IPR im Pre- und Incubation Prozess & $2 \%$ & $29 \%$ & $1.349,98$ & 413,39 \\
\hline Incubation Gründungsbetreuung & $56 \%$ & $100 \%$ & $134.767,05$ & $107.012,56$ \\
\hline HR für Zentrum und Gründung & $7 \%$ & $70 \%$ & $11.368,92$ & $16.489,47$ \\
\hline Alumni & $4 \%$ & $0 \%$ & - & - \\
\hline Finanzierung & $1 \%$ & $90 \%$ & $2.697,81$ & 199,20 \\
\hline Internationalisierung & $3 \%$ & $70 \%$ & $5.218,18$ & $6.364,64$ \\
\hline IPR-Services & $2 \%$ & $0 \%$ & - & 419,22 \\
\hline
\end{tabular}

* KANN-Module: Alumni Betreuung, Finanzierung, Internationalisierung, IPR Services;

Quelle: FFG, Berechnung und Darstellung JR

Es wird deutlich, dass bei einer vorsichtigen Betrachtung jedenfalls ein Drittel der Kosten nicht dem einzelnen Inkubationsfall zugeschrieben werden darf. In einzelnen Fällen liegt dieser Wert auch deutlich darunter. Die Betreuungskosten pro inkubierten Fall liegen (inkl. der vergebenen Förderungen) im Durchschnitt bei rd. 164 Tsd. $€$. Einige Zentren liegen hier in Abhängigkeit von ihrem Aktivitätsportfolio darunter. Es ist festzustellen, dass die Darstellung nur auf Annäherungen basieren kann, da das Monitoring gegenwärtig keine klare Zuordnung bei den Kann-Modulen erlaubt.

Darüber hinaus wurde in den durchgeführten Gesprächen und Fokusgruppen mit Zentren-Leitungen darauf hingewiesen, dass die Darstellung den tatsächlichen Aufwand im Besonderen im Bereich der Bewusstseinsbildung deutlich unterschätzt.

\section{2 Überblick und Leistungsportfolio}

Das Programm wurde 2002 implementiert und zu Beginn mit neun Zentren operativ umgesetzt. 2007 ist ein Zentrum ( $v$-start ${ }^{22}$ ) aus dem Programm ausgeschieden, 2013 wurde ein Ansuchen auf Weiterförderung $\left(\mathrm{BCCS}^{23}\right)$ abgelehnt. 2015 werden somit in sieben regionalen AplusB-Zentren akademische Unternehmensgründungen und Spin-offs unterstützt und gefördert. Das sind accent NÖ, build! Kärnten, CAST Tirol, INiTS Wien, Science Park Graz, tech2b OÖ und ZAT Leoben.

21 Auch hier wurde die monetäre Förderung nicht herausgerechnet.

22 v-start (1.7.2005-30.6.2010) wurde mit 01.07.2010 in die Wirtschafts-Standort Vorarlberg GmbH (WISTO) integriert und fungiert dort weiterhin als Gründungszentrum für High-Tech-Gründungen.

23 BCCS-Business Creation Center Salzburg, Salzburg (01.07.2005 bis 31.12.2014). 
Das Inkubatorennetzwerk Aplus $B^{24}$ ist die nationale und auch internationale Vertretung der regionalen Gründungszentren. Die Administration und Koordination des Programms erfolgt durch die österreichische Forschungsförderungsgesellschaft (FFG).

Die Zentrumsgröße, gemessen an den Beschäftigten, bewegt sich zw. drei (ZAT) und fünfzehn (INiTS) MitarbeiterInnen (siehe Tabelle 3). Zusätzlich zu den Hauptstandorten unterhalten einige der Zentren Büros an anderen Standorten, um so vor Ort professionelle Unterstützung für GründerInnen anbieten zu können (z.B. accent Gründerzentrum Tulln mit der Universität für Bodenkultur), bzw. wurden zuletzt Standorte ausgebaut (z.B. build! Standort Villach).

Tabelle $3 \quad$ Übersicht Zentren, Stand 2015

\begin{tabular}{|c|c|c|c|c|c|c|c|c|c|}
\hline & Accent & BCCS* & build! & CAST & INiTS & SPG & tech2b & ZAT & \multirow[t]{3}{*}{ Gesamt } \\
\hline Gründung & 2005 & 2005 & 2002 & 2002 & 2002 & 2002 & 2002 & 1999 & \\
\hline Hauptstandort & $\begin{array}{c}\text { Wiener } \\
\text { Neustdt. }\end{array}$ & $\begin{array}{l}\text { Slbg. } \\
\text { Stadt }\end{array}$ & $\begin{array}{l}\text { Klagen- } \\
\text { furt }\end{array}$ & $\begin{array}{l}\text { Inns- } \\
\text { bruck }\end{array}$ & Wien & Graz & Linz & Leoben & \\
\hline $\begin{array}{l}\text { Mitarbeiter- } \\
\text { Innen der } \\
\text { Zentren nach } \\
\text { Köpfen gesamt }\end{array}$ & 7 & 4 & 6 & 11 & 15 & 6 & 10 & 3 & 62 \\
\hline $\begin{array}{l}\text { MitarbeiterIn- } \\
\text { nen im Bereich } \\
\text { GründerIn- } \\
\text { nenberatung } \\
\text { nach Vollzeit- } \\
\text { äquivalenten }\end{array}$ & 3,5 & 4 & 2,2 & 3 & 7 & 2,5 & 4,5 & 1 & 25,7 \\
\hline \multicolumn{10}{|l|}{ davon: } \\
\hline $\begin{array}{l}\text { Mitarbeiter- } \\
\text { Innen für } \\
\text { „AplusB } \\
\text { Betreuung“6 } \\
\text { nach Vollzeit- } \\
\text { äquivalenten }\end{array}$ & 3,5 & 4 & 2 & 3 & 7 & 2,5 & 2 & 1 & 23 \\
\hline
\end{tabular}

* Zentrum nicht mehr aktiv.

Quelle: Recherche AplusB-Zentren, Darstellung JR

Als die wichtigsten Ziele und Aufgaben der Zentren werden genannt ${ }^{25}$ :

- ein positives Umfeld für Gründungen schaffen und Gründungsvorhaben anregen;

- $\quad$ spezifisches Gründungs-Know-how vermitteln und Gründungen bis in frühe Unternehmensphasen beraten und begleiten;

- materielle Infrastruktur bereitstellen und Gründungen in relevante Netzwerke integrieren;

- Gründungen bis in frühe Unternehmensphasen mitfinanzieren und Anschlussfinanzierungen vermitteln;

- teilweise umfassende Patentberatungsleistungen.

Die Zentren sind rechtlich gesehen Kapitalgesellschaften (in der Form einer GmbH). Die Gesellschafter setzten sich aus zumindest einer akademischen Einrichtung (Universität, Fachhochschule, außer-

\footnotetext{
24 Siehe: http://www.AplusB.biz

25 AplusB Leistungsbericht 2014, 2013
} 
universitäre Forschungseinrichtung) und einer unbestimmten Anzahl an Partnern zusammen, wie z.B. private Unternehmen, Banken, aber auch Gemeinden oder Länder. Die Gesellschaftsform der GmbH schafft Verbindlichkeit und erlaubt u.a. Vereinfachung in der Förderungsabwicklung.

Diese enge Anbindung an akademische Einrichtungen ist ein Alleinstellungsmerkmal der AplusBZentren im Bereich der Unterstützungslandschaft für Gründungen. Tabelle 4 gibt eine Übersicht über die entsprechenden Gesellschafter-Verhältnisse.

Tabelle $4 \quad$ Übersicht Gesellschafter, Verteilung 2015

\begin{tabular}{|c|c|c|}
\hline Zentrum & Anteil in \% & Gesellschafter \\
\hline \multirow[t]{4}{*}{ Accent } & 30,0 & Donau-Universität Krems \\
\hline & 30,0 & N.vest Unternehmensfinanzierung des Landes NÖ GmbH \\
\hline & 30,0 & RIZ Niederösterreichs Gründeragentur Ges.m.b.H. \\
\hline & 10,0 & NÖ Forschungs- und Bildungsges.m.b.H. \\
\hline \multirow[t]{3}{*}{ BCCS* } & 42,0 & Paris-Lodron-Universität Salzburg \\
\hline & 42,0 & Fachhochschule Salzburg \\
\hline & 16,0 & engage - Key Technology Ventures AG (engage AG Karlsruhe) \\
\hline \multirow[t]{5}{*}{ build! } & 45,0 & BABEG - Ktn. Betriebsansiedlungs- und Beteiligungsgesell. m.b.H. \\
\hline & 30,0 & Alpen-Adria-Universität Klagenfurt \\
\hline & 15,0 & Fachhochschule Kärnten \\
\hline & 5,0 & Stadt Villach \\
\hline & 5,0 & Landeshauptstadt Klagenfurt am Wörthersee \\
\hline \multirow[t]{4}{*}{ CAST } & 30,0 & Universität Innsbruck \\
\hline & 30,0 & Standortagentur Tirol \\
\hline & 25,1 & Medizinische Universität Innsbruck \\
\hline & 14,9 & MCI - Management Center Innsbruck \\
\hline \multirow[t]{3}{*}{ INiTS } & 37,0 & Innovationszentrum Universität Wien $\mathrm{GmbH}$ \\
\hline & 37,0 & Technische Universität Wien \\
\hline & 26,0 & Wirtschaftsagentur Wien \\
\hline \multirow[t]{3}{*}{ SPG } & 50,1 & Technische Universität Graz \\
\hline & 29,3 & Medizinische Universität Graz \\
\hline & 20,6 & Universität Graz \\
\hline \multirow[t]{5}{*}{$\operatorname{tech} 2 \mathbf{b}$} & 55,0 & Business Upper Austria GmbH \\
\hline & 15,0 & Wirtschaftskammer Oberösterreich \\
\hline & 10,0 & Upper Austrian Research GmbH \\
\hline & 10,0 & Johannes Kepler Universität Linz \\
\hline & 10,0 & FH OÖ Management GmbH \\
\hline \multirow[t]{2}{*}{ ZAT } & 50,0 & Montanuniversität Leoben \\
\hline & 50,0 & Stadt Leoben \\
\hline
\end{tabular}

* Zentrum nicht mehr aktiv.

Quelle: Berechnung und Darstellung JR

Die AplusB-GründungsberaterInnen verfügen, wie auch in Einzelgesprächen mit GründerInnen hervorgehoben wurde, über hohe technologische bzw. wirtschaftliche Fachkompetenz, die mit gezielten Qualifizierungsmaßnahmen weiterentwickelt wird, sowie ein breites Erfahrungswissen. Sie widmen 
sich, wie ausnahmslos allen Interviews zu entnehmen ist, mit hohem Engagement und Professionalität der Unterstützung und Entwicklung der potentiellen GründerInnen. Zusätzlich zur Arbeit und dem Austausch mit den GesellschafterInnen verfügen die Zentren über ein erweitertes Netzwerk an Kooperationspartnern, das sich neben dem Forschungs- und Hochschulbereich aus Partnern aus dem Beratungs-, Finanzierungs- und Infrastrukturbereitstellungsbereich sowie anderen privaten Initiativen zusammensetzt. Entsprechend der laufenden Berichterstattung des AplusB-Programms stehen dadurch weit über 150 ExpertInnen für die Anliegen potentieller GründerInnen bereit.

Der Aufbau und die Aufgaben von AplusB-Zentren korrespondieren durchwegs mit den akademischen Trägereinrichtungen und deren Ausrichtung sowie mit der Positionierung einzelner Netzwerkpartner bzw. regionaler Gegebenheiten. Dies bildet sich im etwas häufigeren Auftreten von Gründungsvorhaben in einzelnen Branchen- und Technologiebereichen bei den einzelnen Zentren ab. In jenen Zentren, wo eine stärkere Häufung der Beratung und Unterstützung erkennbar ist, erfolgt dies vorwiegend im Bereich der Informations- und Kommunikationstechnologien (IKT) sowie Life Sciences. Bei Tech2b und dem ZAT ist ein Fokus auf sogenannte „Hardware Gründungen“, etwa im Bereich der Mechatronik, Leichtbau, Werk- und Rohstofftechnik, Produktionstechnik sowie Umwelt- und Energietechnik festzustellen.

Abseits der regionalen Gegebenheiten und Ausrichtung der Partner richten sich die Zentren vor allem an der jeweiligen Entwicklungsphase der, lt. Interviews, hoch-individuellen Gründungsprojekte aus. Vor diesem Hintergrund wurde an Standorten in den letzten Jahren ein spezifischer Maßnahmenmix aufgebaut bzw. weiterentwickelt und ergänzt. Der Kern des AplusB-Programms umfasst nach wie vor eine Inkubator-Funktion, die sich gemäß den AplusB-Richtlinien an die ersten Phasen der Genese und Entwicklung von Unternehmen richtet.

Im Zuge der Implementierung von „AplusB 2.0“ wurde das Leistungsprofil überarbeitet und geschärft. Die Bandbreite reicht nun von Öffentlichkeitsarbeit und Stimulierungs- und Awareness-Maßnahmen, individueller Beratung und Coaching über das Bereitstellen von Infrastruktur bis zu Netzwerkaktivitäten sowie der Finanzierung und Unterstützung von Internationalisierungsprojekten. Während sogenannte „MUSS-Module“ im Wesentlichen das bisherige Leistungsspektrum abdecken, widmen sich „KANN-Module“ Aspekten wie Finanzierung, Internationalisierung oder der Alumni-Betreuung. Diese sind optional und können je nach Größe und Ausstattung des Zentrums bedarfsgerecht in das Leistungsspektrum integriert werden. Zusammengefasst können folgende Bereiche unterscheiden werden:

- Awareness und Coaching (Pre-Inkubation): Bewusstseinsbildende Maßnahmen zur Stimulierung von Gründungen, tlw. auf den jeweiligen Standort bzw. in Abhängigkeit von der jeweiligen Ausrichtung der akademischen Einrichtung zugeschnittenes Portfolio. Dazu zählen Lehrveranstaltungen und Seminare zum Thema Entrepreneurship, Workshops für UniversitätsmitarbeiterInnen und Studierende, Sprechtage an den Forschungseinrichtungen, JungunternehmerInnen- bzw. Gründertage, Businessidee- bzw. Businessplan-Wettbewerbe ${ }^{26}$ und damit verbundene Preise und Awards. Mittels Coaching unterstützen die GründungsberaterInnen und Netzwerkpartner potentielle GründerInnen, etwa bei der Weiterentwicklung der Idee oder der Erstellung eines Geschäftskonzepts. Letzteres wird dem Aufnahmebeirat vorgelegt und entscheidet über den Eintritt in das Zentrum.

- Gründungsbetreuung (Inkubation): Nach erfolgreicher Aufnahme werden die GründerInnen für 18 (bis maximal 24) Monaten in einen - je nach Standort - umfangreichen Betreu-

26 Zum Beispiel Edison, Adventure X, Build! Impuls, INiTS Award, Genius Award, Master Cup oder der SPG Ideenwettbewerb. 
ungs- und Coachingprozess eingebunden. Neben Maßnahmen zur weiteren Aus- und Weiterbildung sowie Qualifizierung in spezifischen Spezialbereichen erhalten die GründerInnen $\mathrm{Zu}$ gang zu Büro- und Forschungsinfrastruktur und dem Netzwerk des Zentrums. Darüber hinaus können AplusB-GründerInnen auch monetäre Förderungen erhalten.

- Alumni-Betreuung (Post-Inkubation): Die Weiterbetreuung von Alumni fällt in den Bereich der „Kann-Module“, d.h. eine systematische Betreuung der aus den Zentren ausgetretenen Unternehmen findet nicht statt. Alumni können hierbei die von AplusB angebotenen, weiterführenden Schulungsmöglichen, die Unterstützung beim Zugang zu Privatkapital sowie die Förderung von Kooperationen bzw. Vernetzung mit anderen Alumni-Teams, der Community, Forschungseinrichtungen oder Unternehmen nutzen. Umgekehrt fungieren Alumni oftmals als Business-MentorInnenen für neu eintretende Projekte oder werden als Role-Model für Veranstaltungen eingeladen, um so einen Erfahrungsaustausch zu pflegen.

Wie bereits im Programm Logic Chart (siehe Kap. 2, Abb. 2) angedeutet, wird hinsichtlich einer allgemeinen Stellung der Zentren im regionalen Gefüge sowie eines konkreten Maßnahmenpaketes für (potentielle) GründerInnen unterschieden (vgl. Abb. 5) Der Bereich „Zentrums- und Öffentlichkeitsarbeit" umfasst allgemeine Awareness-Maßnahmen, Teilnahme an Veranstaltungen und Stimulierungsund Vernetzungsaktivitäten, die über jene mit Fokus auf akademische Einrichtungen hinausgehen, sowie der einheitliche Auftritt der Zentren, etwa als Dachmarke oder Plattform und die Qualifizierung von ZentrumsmitarbeiterInnen für ihre Arbeit mit GründerInnen.

\section{Abbildung 5 Leistungen der AplusB-Zentren}

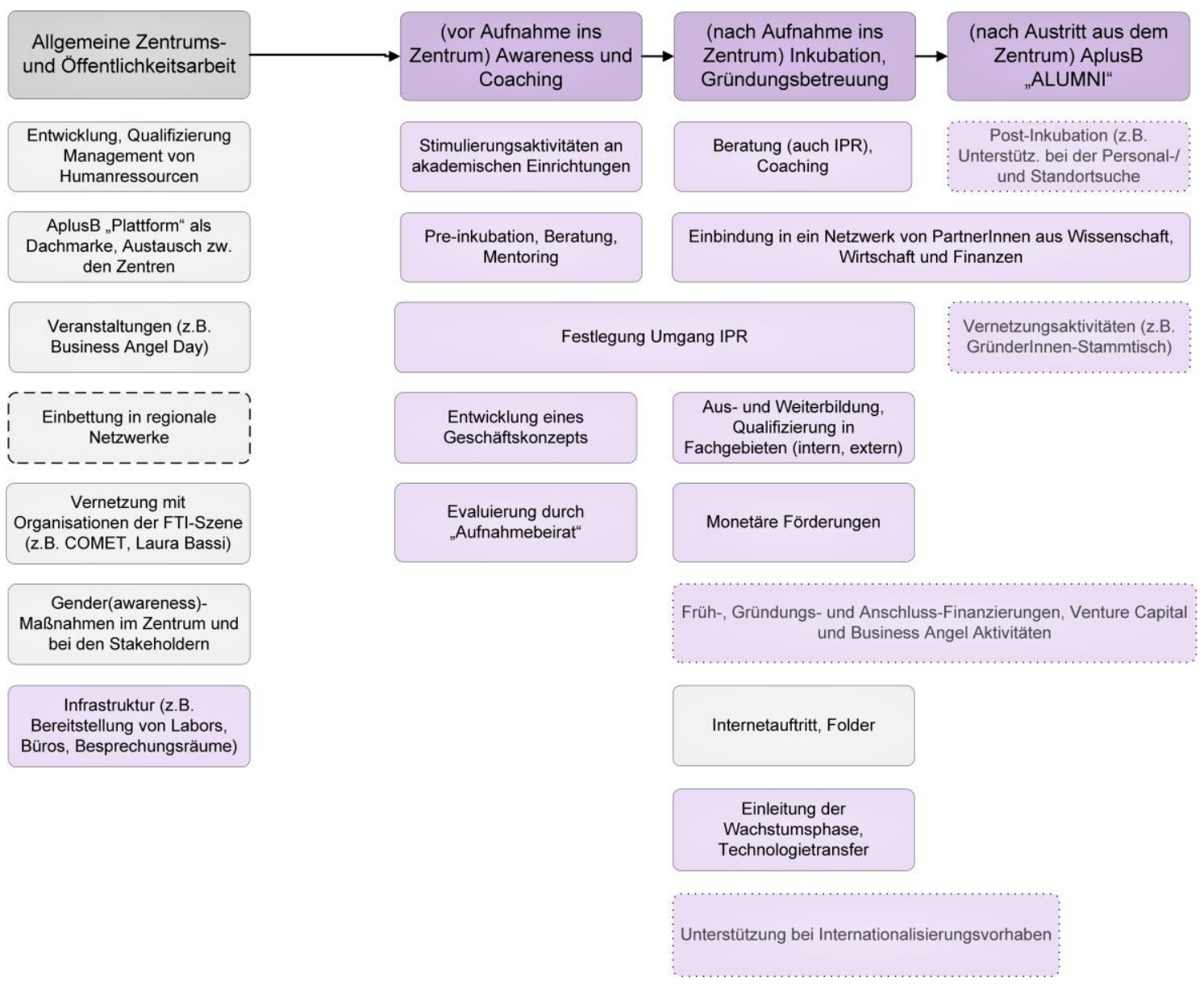

Anmerkung: Gestrichelte Konturen markieren KANN-Module. Quelle: Darstellung: JR 
Zum Leistungsspektrum der Zentren ergeben sich folgende Befunde:

- Wesentlicher Faktor des Leistungsangebots ist die Anzahl und Diversität der vor Ort ansässigen Hochschulen, was unmittelbar Auswirkungen auf die unterschiedlichen Schwerpunktlegungen hat. Dazu zählt auch der Bereich des Technologietransfers. So ist CAST derzeit als einziges Zentrum auch im Wissenstransferzentrum (WTZ) Ost engagiert.

- Auf Grund des gestiegenen Interesses von Seiten dieser Zielgruppen bieten bereits einige AplusBZentren wie build! in Kärnten oder accent in Niederösterreich über eine Non-AplusB Schiene auch Nicht-AkademikerInnen ihre Leistungen an. In Oberösterreich wird in Anlehnung an die Wirtschaftsstruktur eine intensivere Bearbeitung innovativer, aber nicht-akademischer Gründungen geplant. Die Aktivitäten finden formal außerhalb des AplusB-Programms statt. Die Verteilung unter den Gründungsvorhaben, je nachdem ob diese direkt aus der Hochschule oder aus der Privatwirtschaft kommen, ist je nach Standort unterschiedlich.

- Monetäre Förderungen werden an den Zentren vergleichsweise wenig angeboten. Einzelfälle kommen ganz ohne monetäre Förderung aus und fokussieren auf weiche Maßnahmen. Die Unterstützung bei der Finanzmittelsuche gestaltet sich sehr unterschiedlich an den Standorten. In allen Zentren wird jedenfalls Unterstützung bei der Identifikation und Bewerbung bei Förderungsprogrammen geboten. Wie Abschnitt 5.2.2 noch zeigen wird, schlägt sich dies auch positiv in der Präsenz der $A p l u s B$-Gründungen bei diesen Förderungsprogramme nieder.

- Das Thema gewerbliche Schutzrechte (Intellectual Property Rights, IPR) wird von den Zentren verstärkt aufgegriffen. Hierfür existieren in den unterschiedlichen Betreuungsphasen verschiedene Angebote, wie z.B. IP-Beratung und Trainings oder die Vermittlung an ein Netzwerk von IPExpertInnen für Patentierung und Lizensierung.

- Einige Zentren betreuen Gründungen aus dem Ausland, die vor Ort, z.B. CAST (Deutschland) oder build! (Slowenien, Italien), unterstützt und betreut werden.

In den letzten Jahren widmen sich die Zentren verstärkt Themen wie Geschlechtervielfalt und Diversität als Erfolgsfaktoren für die Gründungsteams. Die österreichweit eingerichtete Arbeitsgruppe „Gender" des AplusB-Vereins unterstützt dabei die Zentren, um Gender Mainstreaming in den gelebten Alltag zu integrieren. Die Broschüre AplusB $+\mathrm{C}$ berichtet über Forscherinnen und Technikerinnen in ihrer Rolle als Unternehmerinnen. An gendergerechten Angeboten für Frauen, beispielsweise Teilnahme am Töchtertag, Angebot von Strategieworkshops für Frauen in Kooperation mit w-fFORTE und vielen mehr, wird gearbeitet.

\subsection{AplusB im internationalen Umfeld}

Die Unterstützung von innovativen Gründungen hat nicht nur in Österreich, sondern auch im internationalen Umfeld an Bedeutung gewonnen. Dementsprechend dynamisch hat sich die Zahl der Inkubatoren in Europa in den vergangenen zwei Jahrzehnten vervielfacht. Das AplusB-Programm wird dabei auch immer wieder als Beispiel im internationalen Umfeld herangezogen. Stellvertretend für das $A p$ lusB-Programm gelang es dem INITS als größtes Zentrum in den vergangenen Jahren wiederholt, internationale Anerkennung zu erhalten, indem es unter den global Top-25 universitärer Inkubatoren gewählt wurde. ${ }^{27}$ Ein Großteil der Inkubatoren und Inkubatoren-Programme wird national finanziert.

27 Das INITS wurde von der schwedischen Forschungsinitiative UBI Index in das Ranking der „Global Top 25“ die universitären Inkubatoren aufgenommen und belegt den weltweit 11. Platz, sowie den 3. Platz in Europa. 
In zahlreichen Fällen erfolgt auch eine Kofinanzierung durch EU-Mittel (EFRE) ${ }^{28}$ Die förderverantwortlichen Stellen sind in der Regel Ministerien für Forschung, Technologie und Wirtschaftsangelegenheiten bzw. delegierte Förderagenturen. Die von öffentlichen Programmen unterstützten Inkubatoren weisen in der Regel eine öffentliche Trägerschaft auf.

Tabelle $5 \quad$ Inkubatoren-Typen im Vergleich

\begin{tabular}{|c|c|c|c|c|}
\hline Тур & $\begin{array}{l}\text { A) Inkubatoren der } \\
\text { Wirtschaftsförde- } \\
\text { rung }\end{array}$ & $\begin{array}{l}\text { B) Inkubatoren für } \\
\text { akademische oder } \\
\text { innovative Grün- } \\
\text { dungen }\end{array}$ & $\begin{array}{l}\text { C) Private Inkuba- } \\
\text { toren in Unter- } \\
\text { nehmen } \\
\text { (-sgruppen) }\end{array}$ & $\begin{array}{ll}\text { D) Private } \\
\text { Investoren }\end{array}$ \\
\hline & Non profit & Non profit & $\begin{array}{l}\text { For profit, } \\
\text { unternehmensgebunden }\end{array}$ & $\begin{array}{l}\text { For profit, } \\
\text { „unabhängig“" }\end{array}$ \\
\hline Fokus & $\begin{array}{l}\text { Allgemein; nicht akade- } \\
\text { misch }\end{array}$ & $\begin{array}{l}\text { technologieintensiv; } \\
\text { akademisch }\end{array}$ & $\begin{array}{l}\text { technologie- u. wissens- } \\
\text { intensiv }\end{array}$ & $\begin{array}{l}\text { technologie- } u \text {. wissens- } \\
\text { intensiv }\end{array}$ \\
\hline Ziele & $\begin{array}{l}\text { Schaffung von Beschäfti- } \\
\text { gung, Regional- u. Wirt- } \\
\text { schaftsentwicklung, } \\
\text { Sektorale Entwicklung, } \\
\text { KMU, Cluster }\end{array}$ & $\begin{array}{l}\text { Kommerzialisierung von } \\
\text { Technologien und For- } \\
\text { schungsergebnissen, } \\
\text { Einbeziehung von Fakul- } \\
\text { tätsmitarbeiterInnen } \\
\text { /Studierenden; Entwick- } \\
\text { lung von Unternehmer- } \\
\text { geist, Missionsorientie- } \\
\text { rung, innovative Finan- } \\
\text { zierungsformen }\end{array}$ & $\begin{array}{l}\text { Förderung von Unter- } \\
\text { nehmergeist der Mitar- } \\
\text { beiterInnen, Zugang zu } \\
\text { neuen Technologien und } \\
\text { Märkten, Diversifikation } \\
\text { der Geschäftstätigkeit, } \\
\text { Profit }\end{array}$ & $\begin{array}{l}\text { Streuung des Investiti- } \\
\text { onsrisikos, Renditenma- } \\
\text { xierung durch die Portfo- } \\
\text { lio Performance, günsti- } \\
\text { ger Erwerb von IPRs, } \\
\text { Austausch zwischen } \\
\text { Unternehmen im Portfo- } \\
\text { lio }\end{array}$ \\
\hline $\begin{array}{l}\text { Ziel- } \\
\text { gruppe }\end{array}$ & $\begin{array}{l}\text { Gewerbliche Gründungs- } \\
\text { vorhaben }\end{array}$ & $\begin{array}{l}\text { Innovative Geschäftsi- } \\
\text { deen in der Frühphase }\end{array}$ & $\begin{array}{l}\text { Spin-offs aus bestehen- } \\
\text { den Geschäftsbereichen }\end{array}$ & Reifere Geschäftsmodelle \\
\hline Phase & Start-up & Idee, Start-up & Expansion & Start-up; Expansion \\
\hline $\begin{array}{l}\text { Leistun- } \\
\text { gen }\end{array}$ & $\begin{array}{l}\text { Beherbergung, gemein- } \\
\text { same Nutzung von Infra- } \\
\text { struktur, administrative } \\
\text { Unterstützung, Beratung, } \\
\text { Coaching, Weiterbildung, } \\
\text { Vernetzung, Zugang zu } \\
\text { Finanzierung u. Förder- } \\
\text { beratung }\end{array}$ & $\begin{array}{l}\text { Konzeptprüfung, techni- } \\
\text { sche Hilfestellung, IP- } \\
\text { Management, (strategi- } \\
\text { sche) Beratung, } \\
\text { Coaching, Zugang zu } \\
\text { industriellen Netzwerken, } \\
\text { Zugang zu Business } \\
\text { Angels (od. VC), Förder- } \\
\text { beratung, Beherbergung }\end{array}$ & $\begin{array}{l}\text { Bereitstellung von Fi- } \\
\text { nanzressourcen, Prototy- } \\
\text { ping und Markttest, } \\
\text { Zugang zu Vertriebska- } \\
\text { nälen, langfristige strate- } \\
\text { gische Partnerschaft }\end{array}$ & $\begin{array}{l}\text { Strategische Beratung, } \\
\text { rechtlichen Support, } \\
\text { Finanzierung und Bera- } \\
\text { tung zu komplementärer } \\
\text { Finanzierung und Förde- } \\
\text { rung, persönliche Netz- } \\
\text { werke, Beherbergung und } \\
\text { administrative Unterstüt- } \\
\text { zung }\end{array}$ \\
\hline
\end{tabular}

Quelle: Spath and Walter 2012, Erk O. 2006, Kolo J., Al-Mubaraki H. M. 2012, Darstellung JR

Das ITAY-Beck-Programm in Israel stellt ein Beispiel dar, wo Inkubatoren im Privatbesitz unter festgelegten Bedingung und der Einbringung von Eigenmitteln eine Förderung erhalten.

Die Inkubatoren-Landschaft ist so vielfältig wie auch die Trägerschaft und die angestrebten Zielgruppen. Im Wesentlichen lassen sich vier Typen unterscheiden, wobei die AplusB-Zentren dem im folgenden Überblick in der zweiten Spalte dargestellten Typ B am nächsten kommen.

Im internationalen „Inkubatoren-Geschehen“ sind darüber hinaus einige wenige Trends zu beobachten, die einer kurzen Erwähnung verdienen. Inkubatoren positionieren sich in unterschiedlichen Phasen, dies gilt im Besonderen für die Differenzierung zwischen öffentlich getragenen Non-Profit und privaten Inkubatoren. Die folgende Übersicht zeigt eine Reihe von Beispielen und deren Positionierung entsprechend der gesetzten Ziele, der Fokussierung auf einzelne Phasen der Gründung und den eingesetzten Instrumente.

28 Ein Überblick findet sich unter: https://ec.europa.eu/growth/tools-databases/regional-innovation-monitor/keywords/start-upsspinoffs 
Tabelle $6 \quad$ Positionierung ausgewählter internationaler Inkubatorenprogramme

\begin{tabular}{|c|c|c|c|c|c|}
\hline Name (Land) & Start & Тур & $\begin{array}{l}\text { Übergeordnetes } \\
\text { Ziel }\end{array}$ & $\begin{array}{l}\text { Phase; } \\
\text { Unterstuitzungs- } \\
\text { zeitraum } \\
\end{array}$ & Instrumente \\
\hline $\begin{array}{l}\text { EXIST IV - } \\
\text { Gründerhoch- } \\
\text { schule (Deutsch- } \\
\text { land) } \\
\end{array}$ & 2010 & $\begin{array}{l}\text { Öffentlich, Non- } \\
\text { Profit, Fokus auf } \\
\text { akad. Gründungen }\end{array}$ & $\begin{array}{l}\text { Gründungskultur u. } \\
\text { Unternehmergeist } \\
\text { an Hochschulen } \\
\text { etablieren }\end{array}$ & $\begin{array}{l}\text { Early stage, start- } \\
\text { up; } \\
\mathbf{3} \text { (+2 Jahre) }\end{array}$ & $\begin{array}{l}\text { Inkubation, Fi- } \\
\text { nanzmittel, Mentor- } \\
\text { ing }\end{array}$ \\
\hline $\begin{array}{l}\text { Incubation et } \\
\text { capital amorçage } \\
\text { des entreprises } \\
\text { technologiques } \\
\text { (Frankreich) }\end{array}$ & 1999 & $\begin{array}{l}\text { Öffentlich, n Non- } \\
\text { Profit, Fokus auf } \\
\text { akad. Gründungen }\end{array}$ & $\begin{array}{l}\text { Inkubator-System } \\
\text { mit Fokus auf Ver- } \\
\text { bindung zw. } \\
\text { Academia und } \\
\text { Inkubatoren }\end{array}$ & $\begin{array}{l}\text { Start-up; } \\
\text { 3 Jahre }\end{array}$ & $\begin{array}{l}\text { Inkubation: Bera- } \\
\text { tung, Infrastruktur }\end{array}$ \\
\hline $\begin{array}{l}\text { ITAY Beck - } \\
\text { Technologisches } \\
\text { Inkubatorpro- } \\
\text { gramm } \\
\text { (Israel) }\end{array}$ & 1991 & $\begin{array}{l}\text { Öffentlich, Non- } \\
\text { Profit, Fokus auf } \\
\text { akad. Gründungen }\end{array}$ & $\begin{array}{l}\text { Abstrakte Ideen in } \\
\text { technisch machbare, } \\
\text { neuartige Produkte } \\
\text { zu überführen }\end{array}$ & $\begin{array}{l}\text { Early stage, start up; } \\
\mathbf{2} \text { max. } 3 \text { Jahre }\end{array}$ & $\begin{array}{l}\text { Inkubation: Räum- } \\
\text { lichkeiten, Finanz- } \\
\text { mittel, Infrastruktur, } \\
\text { Coaching, administ- } \\
\text { rative Unterstützung }\end{array}$ \\
\hline $\begin{array}{l}\text { IUNet - Incubato- } \\
\text { ri Universitari } \\
\text { Network } \\
\text { (Italien) }\end{array}$ & 2004 & $\begin{array}{l}\text { PPP (Inkubatoren } \\
\text { bringen zur Förde- } \\
\text { rung Eigenmittel } \\
\text { ein), Fokus auf } \\
\text { akad. Gründungen }\end{array}$ & $\begin{array}{l}\text { wirtschaftliche und } \\
\text { soziale Entwicklung } \\
\text { in Italien durch } \\
\text { Förderung von } \\
\text { Innovation u. Entre- } \\
\text { preneurship }\end{array}$ & Early stage, start up & $\begin{array}{l}\text { Virtuelle Inkubati- } \\
\text { on: technologische } \\
\text { und organisatori- } \\
\text { sche Beratung, } \\
\text { virtuelle Office- } \\
\text { services }\end{array}$ \\
\hline $\begin{array}{l}\text { NIP1 - National } \\
\text { Incubator Pro- } \\
\text { gramme (Pilot- } \\
\text { phase) } \\
\text { (Schweden) }\end{array}$ & 2003 & $\begin{array}{l}\text { Öffentlich, Non- } \\
\text { Profit, Fokus auf } \\
\text { wirtschaftl. Ent- } \\
\text { wicklung }\end{array}$ & $\begin{array}{l}\text { Innovationsförde- } \\
\text { rung }\end{array}$ & $\begin{array}{l}\text { Start up; } \\
18 \text { Monate }\end{array}$ & $\begin{array}{l}\text { Inkubation: Man- } \\
\text { agement-Training, } \\
\text { Teambildung, } \\
\text { Coaching, Business } \\
\text { Developm. (Start-up } \\
\text { \& Inkubator) }\end{array}$ \\
\hline $\begin{array}{l}\text { Support Pro- } \\
\text { gramms of Enter- } \\
\text { prise Ireland } \\
\text { (Irland) }\end{array}$ & 1999 & $\begin{array}{l}\text { Öffentlich, Non- } \\
\text { Profit, Fokus auf } \\
\text { wirtschaftl. Ent- } \\
\text { wicklung }\end{array}$ & $\begin{array}{l}\text { Arbeitsbeschaffung, } \\
\text { Innovationsförde- } \\
\text { rung }\end{array}$ & start up, Expansion & $\begin{array}{l}\text { Subventionen, } \\
\text { Steuerverminde- } \\
\text { rung, VC, Unter- } \\
\text { nehmer-Ausbildung, } \\
\text { Management- } \\
\text { Beratung }\end{array}$ \\
\hline $\begin{array}{l}\text { New Frontiers } \\
\text { Entrepreneur } \\
\text { Development } \\
\text { Programme } \\
\text { (Irland) } \\
\end{array}$ & 2012 & $\begin{array}{l}\text { Öffentlich, Non- } \\
\text { Profit Fokus auf } \\
\text { innovative Grün- } \\
\text { dungen }\end{array}$ & $\begin{array}{l}\text { Innovationsförde- } \\
\text { rung }\end{array}$ & Early stage, start up & $\begin{array}{l}\text { Inkubation, Mento- } \\
\text { ring, Stipendium }\end{array}$ \\
\hline $\begin{array}{l}\text { Start-up Chile } \\
\text { (Chile) }\end{array}$ & 2010 & $\begin{array}{l}\text { Öffentlich, Non- } \\
\text { Profit, Fokus auf } \\
\text { akad. Gründungen }\end{array}$ & $\begin{array}{l}\text { Wettbewerbsfähig- } \\
\text { keit durch Innovati- } \\
\text { on u. Entrepreneu- } \\
\text { rship; Maßnahmen } \\
\text { gegen den Brain } \\
\text { Drain von Talenten }\end{array}$ & $\begin{array}{l}\text { Early stage; } \\
6 \text { Monate }\end{array}$ & $\begin{array}{l}\text { Stipendien, Bereit- } \\
\text { stellung von Kon- } \\
\text { takten, Visum }\end{array}$ \\
\hline $\begin{array}{l}\begin{array}{l}\text { StartupHighway } \\
\text { (Litauen) }\end{array} \\
\end{array}$ & 2011 & $\begin{array}{l}\text { Öffentlich, Non- } \\
\text { Profit, Fokus auf } \\
\text { wirtschaftl. Ent- } \\
\text { wicklung }\end{array}$ & $\begin{array}{l}\text { Arbeitsbeschaffung, } \\
\text { Innovationsförde- } \\
\text { rung }\end{array}$ & Alle Phasen & $\begin{array}{l}\text { Stipendien, Rechts- } \\
\text { und Management- } \\
\text { Beratung, Infra- } \\
\text { struktur }\end{array}$ \\
\hline
\end{tabular}

Quelle: Darstellung JR

Die Inkubatoren-Szene entwickelt ihre Angebote schrittweise weiter. Im Bereich der Inkubatoren, die mit innovativen technologieorientierten Gründungen arbeiten, werden die Möglichkeiten sozialer Netzwerke, die Partnerschaft mit universitären oder klinischen Forschungszentren oder auch der Austausch auf der Ebene der GründerInnen zwischen Partnerinkubatoren zunehmend forciert. Auch die AplusB-Zentren bauen direkte Partnerschaften zu Dritten zunehmend auf. Der Zentren-übergreifende Austausch erfolgt gegenwärtig vorrangig auf der Ebene der Zentren-Managements, aber nicht auf der Ebene der Gründungen. 
Die Betreuungsdauer in Inkubatoren ist im internationalen Umfeld unterschiedlich. Das AplusBProgramm ist mit seinen 18 Monaten im unteren Segment angesiedelt. Im mittelfristigen Trend ist eine zunehmende Erweiterung der Horizonte festzustellen, bspw. in Richtung der Früh- oder auch Reifephasen. In etwa seit 2010 wird - inspiriert von Beispielen in den USA und begleitet durch eine Diskussion auf europäischer Ebene - Akzeleratoren größere Aufmerksamkeit gewidmet. Erste Erfahrungen zeigen allerdings, dass dieses auf reifere Phasen nach der Gründung hin fokussierte Modell aufgrund unterschiedlicher regulativer und struktureller Rahmenbedingungen nicht so einfach in den europäischen Kontext transferierbar ist ${ }^{29}$.

In Abhängigkeit vom Leistungsportfolio ergibt sich eine sehr unterschiedliche Betreuungsintensität. Die Zahl der MitarbeiterInnen hängt unter anderem aber auch von der Größe des einzelnen Inkubators ab: In kleineren Einheiten fallen in der Regel mehr MitarbeiterInnen pro Betreuungsfall an. Dies zeigt eine jüngst veröffentliche Erhebung der acatech im Bereich deutscher Inkubatoren, die sich in einem ähnlichen Umfeld wie AplusB bewegen. Dabei wird deutlich, dass AplusB ein Netzwerk relativ kleiner Inkubatoren darstellt. Lediglich $15 \%$ der im Rahmen der acatech-Studie betrachteten Inkubatoren betreut zehn oder gar weniger Fälle gleichzeitig. Der durchschnittliche Wert liegt sogar bei 47 gleichzeitig betreuten Fällen. Die im Rahmen der acatech-Studie festgestellte durchschnittliche Betreuungsdichte (Zahl der MitarbeiterInnen pro Betreuungsfall) beläuft sich auf 0,23. Unter Berücksichtigung der in diesem Zusammenhang festgestellten Standardabweichung von 0,33 ist die Betreuungsdichte der AplusB-Zentren dennoch deutlich höher als beim Durchschnitt der betrachteten Zentren.

Tabelle $7 \quad$ Betreuungsdichte im Vergleich

\begin{tabular}{|l|c|c|}
\hline Referenz & Durchschnittlich betreute Projekte & $\begin{array}{l}\text { GründungsberaterInnen } \\
\text { pro Betreuungsfall }\end{array}$ \\
\hline Inkubatorenerhebung Deutschland & 46,90 & 0,23 \\
\hline AplusB gesamt & 10 & $0,46^{30}$ \\
\hline
\end{tabular}

Quelle: Spath und Walter $2012^{31}$, Darstellung JR

Die Betreuungsdichte schlägt sich implizit auch in den Kosten je realisierter Gründung nieder. Dennoch kann daraufhin kein direkter Zusammenhang hergestellt werden.

Soweit hierfür Daten vorliegen, kann sich das AplusB-Programm sehr gut mit den identifizierten Beispielen messen. Mit einer Überlebensrate von 93 \% im dritten Jahr nach der Gründungen (bspw. EXIST IV $83 \%$, Incubation et capital amorçage des entreprises technologiques $88 \%$ ) und sechs geschaffenen Arbeitsplätzen im dritten Jahr (siehe Incubation et capital amorçage des entreprises technologiques: 4,3; IUNet - Incubatori Universitari Network: 5,3) kann sich das AplusB durchaus im Vergleich ähnlicher Programme messen.

Für einen angemessenen Vergleich der Kosten pro Gründungsfall liegen keine Datengrundlagen vor. Dies begründet sich damit, dass die einzelnen Inkubatoren unterschiedliche Phasen der Gründung begleiten. Des Weiteren liegt keine hinreichende Aufschlüsselung von direkten und indirekten Kosten vor. Diese sind sehr stark davon abhängig, inwieweit Zentren auch Aufgaben im Bereich der öffentlichen Bewusstseinsbildung und Vorbereitung bzw. Sensibilisierung von potentiellen GründerInnen

29 WBCInno 2014

30 Der Wert 0,7 wurde anhand der festgestellten MitarbeiterInnen und Betreuungsfälle der Zentren sowie des geschätzten Anteils der Inkubationsarbeit angenähert.

31 Spath, D.; Walter, A.; (2012): Mehr Innovationen für Deutschland. Wie Inkubatoren akademische Hightech-Ausgründungen besser fördern können: acatech - Deutsche Akademie der Technikwissenschaften, 201. 
übernehmen. Das AplusB-Programm und seine Zentren widmen dem gemeinsam mit den Hochschulen bewusst vergleichsweise hohe Aufmerksamkeit. Ein weiterer Punkt betrifft die Vergabe von Förderungsmitteln (bzw. seed-financing) über Inkubatoren, welche zu weiteren Verzerrungen führt.

Ordnet man die Beanspruchung einzelner Leistungsmodule der AplusB-Zentren den Gründungen zu, so ergibt sich ein Durchschnittswert von $164.000 €$ pro Gründung, in einzelnen Fällen deutlich darunter $(137.000 €)$. Eine exakte Gegenüberstellung bedarf detaillierter Daten von Seiten der internationalen Beispiele, die nicht zur Verfügung gestellt werden konnten. Es wird aber jedenfalls deutlich, dass das AplusB-Programm betreffend den Kosten nicht aus dem Rahmen fällt.

Tabelle 8 Aufwendungen pro Gründungen im Vergleich

\begin{tabular}{|l|c|c|}
\hline Beispielzentren & $\begin{array}{l}\text { Durchschnittlich } \\
\text { betreute Projekte }\end{array}$ & $\begin{array}{l}\text { Aufwendungen pro } \\
\text { erfolgreicher Gründung } \\
\text { in } €\end{array}$ \\
\hline $\begin{array}{l}\text { Incubation et capital amorçage des entreprises technologiques } \\
\text { (F) }\end{array}$ & $>100$ & $136.000 €$ \\
\hline IUNet - Incubatori Universitari Network (virtuelle Inkubation) & 50 & $110.000 €$ \\
\hline AplusB-Zentren & 50 & $164.000 €$ \\
& & (min. $137.000 €)$ \\
\hline
\end{tabular}

Quelle: eigene Berechnungn

Die Betrachtung vergleichbarer Inkubatoren (Programme) mit dem AplusB-Programm zeigt, dass das Programm in Hinblick auf seine Ausrichtung und auch die Verankerung in der Politiklandschaft durchaus vergleichbar mit anderen Programmen ist und sich auf einem sehr hohen Niveau mit diesen messen kann. Dies gilt sowohl in Hinblick auf die ökonomischen Wirkungen (Überlebensrate, Beschäftigung) als auch in Hinblick auf Umfang und Qualität der Leistungen, die erbracht werden.

Mit der angestrebten Verankerung in den regionalen Innovations- und Politiksystemen sind die $A p$ lusB-Zentren auch kleiner strukturiert als der Durchschnitt der Inkubatoren in Deutschland. Ein direkter Vergleich ist nicht möglich, auch weil in sehr unterschiedlichem Ausmaß über die Inkubation hinausgehende Aktivitäten durchgeführt werden.

Es kann jedenfalls festgestellt werden, dass, soweit Daten verfügbar sind, das AplusB-Programm sich hinsichtlich der Kosten pro Gründung in einem mit anderen Programmen in Europa vergleichbaren Rahmen bewegt. Dies gilt insbesondere, wenn die unter anderem nach den erfolgten Rechnungshofprüfungen ohnehin eingeleiteten Maßnahmen zur Kosteneinsparung u.a. im Bereich der Betreuungsdichte realisiert werden.

\subsection{Bewertung der entwickelten Strukturen und Umsetzung}

- Ermöglicht die existierende Gesamtorganisation des Programms eine effiziente und effektive Umsetzung der Mission und Zielsetzungen? Welche Optimierungspotentiale bei Organisation und Management ergeben sich, welche Erfordernisse sind für deren Erreichung notwendig?

Das AplusB-Programm hat sich die flächendeckende Unterstützung von akademischen, innovativen und wachstumsorientierten Gründungen in einem durchaus heterogenen Innovationsumfeld zum Ziel gesetzt. Die Voraussetzungen für die Initiierung von derartigen Gründungen sind in den einzelnen Bundesländern und Forschungsstandorten sehr unterschiedlich. Die Rolle des Bundes und einer nationalen Abstimmung begründet sich mit der Orientierung an internationaler Wettbewerbsfähigkeit und Standards, überregionaler Mobilität und Einbindung der Hochschulen. 
Es ist vorgesehen, dass die AplusB-Zentren einen wesentlichen Teil der Abstimmung zwischen regionalen Bedarfen und Politik und dem Gesamtprogramm AplusB bewältigen. Diese Abstimmung bedarf intensiver Kommunikation und funktioniert im Wesentlichen auch. Für die Zentren und auch die Gründungen ist es entscheidend, dass unterschiedliche Stakeholder einander die Balance halten. Dies fordert die Abstimmung von Interessen im Innenverhältnis und stellt eine gewisse Offenheit und Flexibilität nach außen sicher. Aus der Sicht der EvaluatorInnen sollen standortübergreifende Mobilität und Zugriff auf AplusB-Leistungen durch Gründungen erhalten bleiben und auch forciert werden.

Ungeachtet der positiv wahrgenommenen Abstimmung vor Ort durch die Zentren kam in den durchgeführten Fokusgruppen von Seiten der Förderungsstellen und regionalen Stakeholder zum Ausdruck, dass jährliche Gespräche (ähnlich der Vorgehensweise beim Programm COMET) von großem Nutzen und erwünscht wären.

- Folgen Inhalte, Design und Abläufe der Prozesse bei der FFG den Prinzipien der Transparenz und Fairness und sind sie den Zielen von AplusB angemessen?

Das Programmmanagement der FFG wird als schlank in den Strukturen, effizient und transparent wahrgenommen. Dies gilt für die Abwicklung der Ausschreibungs- und Bewilligungsprozesse sowie die Evaluierungsprozesse. Die Kommunikation durch das Programmmanagement wird positiv wahrgenommen. Bezugnehmend auf das Monitoring und Berichtswesen sind allerdings Anknüpfungspunkte für Verbesserungen festzustellen.

Die Evaluierungen der Einzelnen Zentren erfolgten zuletzt auf der Grundlage der umfangreichen Materialien, die von den AplusB-zentren und der FFG zur Verfügung gestellt wurden. Die zuletzt durchgeführte Ausschreibung und Auswahl wurde als Standard Ausschreibung der FFG durchgeführt (Modell 4) durchgeführt. Dies schließt eine Jurysitzung inkl. Hearing der Antragsteller (GeschäftsführerInnen mit max. zwei weiteren VertreterInnen) ein. Die hierbei einbezogenen Unterlagen waren der Antrag (Rückschau - Vorschau), Auswertungen zum Gründungs-Monitoring sowie Zusammenfassungen der Drei- und Fünf-Jahresevaluierungen. Ein Lokalaugenschein durch die EvaluatorInnen der Zentren oder umfangreichere Befragungen von GründerInnen oder Stakeholdern wurden nicht vorgenommen. Vor-Ort-Besuche der EvaluatorInnen sind bei den Zwischenevaluierungen vorgesehen.

- Wie haben sich die Auswahl- und Evaluierungskonzepte (Prozesse, Abwicklung, Beiratsmitglieder) bewährt? Wie werden sie hinsichtlich Kompetenz/Expertise, Neutralität und Effizienz beurteilt?

Die Koordination der AplusB-Zentren stellt nach außen hin ein Signal in Richtung Unabhängigkeit und Transparenz der Auswahlprozesse dar. Dies setzt eine entsprechende Dokumentation der Entscheidungen voraus. Die Zentren haben unterschiedliche Modelle entwickelt, um eine effektive und vor dem Hintergrund des erheblichen Ressourcenaufwands effiziente Auswahl zu treffen, von denen sich mittelfristig ein „Good Practice“-Modell ableiten ließe.

Die Auswahl von Gründungsvorhaben werden anerkannt und verantwortungsvoll in einem strukturieren und nachvollziehbaren Selektionsprozess umgesetzt. In den Gesprächen mit (erfolgreich aufgenommen) Start-ups wurde der Aufnahmeprozess überwiegend als aufwendig und umfangreich, mitunter als bürokratisch, letztlich aber als fair und sachlich empfunden. Das hohe Detailwissen der Expertinnen wurde generell als positiv gesehen. Deren Hintergrund, der in einigen Zentren in klassischen Industriebetrieben verortet ist, wurde fallweise als wenig kompatibel mit dem Funktionieren eines Start-ups gesehen.

Die Mitglieder des Projekt- bzw. Vergabebeirat setzen sich in den einzelnen Zentren aus nationalen wie internationalen ExpertInnen mit langjähriger Erfahrung und Spezialwissen in der Branche zusam- 
men. Über die Aufnahme von GründerInnen entscheidet letztlich der bzw. die GeschäftsführerIn des Zentrums.

Die Aufnahme von Kooperations- bzw. Netzwerkpartner der Zentren in den Projektbeirat erscheint unter dem Gesichtspunkt der regionalen und akademischen Anbindung sinnvoll. Die Neutralität wird dadurch gewahrt, dass die Zentren nicht an der Wertsteigerung der Unternehmen, etwa in der Form von Beteiligungen, partizipieren. Dennoch sollte unter dem Gesichtspunkt des Interessensausgleiches überlegt werden, den Austausch von Personen in den Vergabegremien zwischen den Zentren zu intensivieren und, soweit möglich und vertretbar, Personen, die selbst in Start-ups mitgearbeitet haben bzw. verstärkt internationale ExpertInnen für Projektbewertungen zu gewinnen.

- Konnte bei der Abwicklung durch die FFG ein sinnvolles Gleichgewicht zwischen Informationsund Kontrollbedarf auf der einen Seite, Unterstützung und Coaching der Zentren auf der anderen Seite erreicht werden? Welche Qualitätssicherungsmechanismen werden (neben dem GründungsMonitoring und den FFG-Controllings) genutzt und sind diese ausreichend?

Im Laufe der Programmimplementierung wurde ein sehr ausdifferenziertes Controlling und Monitoring-Instrumentarium entwickelt, dass routiniert umgesetzt wird, allerdings in einigen Punkten anpassungsbedürftig erscheint.

- Das Gründungs-Monitoring knüpft sehr stark an der Befragung der GründerInnen an und sollte in seiner Umsetzung eine Umlegung auf die Unternehmenseinheit begünstigen. Des Weiteren erlaubt es nur eingeschränkt die Unternehmensentwicklung zu verfolgen.

- Das Berichtswesen ist sehr umfangreich und detailliert. Die umfangreiche schriftliche Dokumentation sollte in Teilbereich auf ein schlankes Monitoringsystem in Anlehnung an eine Balanced Scorecard (BSC) umgelegt werden, das auch in der Lage ist, Outputs und Outcomes in einer vergleichbaren Form (z.B. monatsaktuell) zu erfassen. Dies erlaubt eine Reduktion der Zahl und des Umfangs der Statusberichte.

- In der Darstellung von Aufwendungen sollte zwischen direkten und indirekten Kosten differenziert werden und die mehrfache Zuordnung zu Aktivitäten bzw. Kostenträgern begünstigt werden.

Neben dem Gründungs-Monitoring und dem Berichtswesen werden auf jährlicher Basis Prüfungen der Zentren durch die FFG durchgeführt. Diese Prüfungen umfassen u.a. die Überprüfung der förderbaren Kosten, Finanzierungsquoten im Prüfungszeitraum, die Feststellung des Organisationsaufbaus und ablaufs sowie ein Monitoring der Tätigkeiten und Maßnahmen des Zentrums.

- Wie werden die Leistungen des Programmmanagements hinsichtlich Effektivität, Effizienz und Kundenzufriedenheit beurteilt?

Das Programmmanagement bei der FFG ist sehr schlank aufgebaut und deckt die Grundfunktionen ab. Die eingerichtete Plattform ist daher auch in Hinblick auf die strategische und inhaltliche Begleitung des Programms und der Zentren unentbehrlich geworden. Eine Reduktion des administrativen Aufwands, wie oben erwähnt etwa durch die Verschlankung der Reporting-Strukturen, sollte nach Maßgabe geprüft werden. Zudem bedürften die Berichts- und Monitoring-Instrumente inhaltlich einer Revision, wie die direkte Einsichtnahme sowie auch Interviews und Fokusgruppen bestätigen.

- (Aus Programmperspektive:) Wie positioniert sich das AplusB-Programm in Relation zu vergleichbaren privaten Initiativen?

Unterschiedliche Typen von Inkubatoren verfolgen unterschiedliche, im Wesentlichen komplementäre Ziele unter unterschiedlichen Rahmenbedingungen, wie aus den angestellten Betrachtungen klar hervorgeht. Das Programm erlaubt auf der Ebene der einzelnen Zentren eine Anpassung an das regionale 
Unterstützungsumfeld. Wie einige der Interviews nahelegen ist die Möglichkeit, sich mit privaten Initiativen und Angeboten, an den verschiedenen Standorten recht unterschiedlich. Während einige der Zentren das AplusB-Angebot in einen Gesamtprozess von unterschiedlichen privaten wie öffentlichen Angeboten eingebettet, fehlt es an anderen Standorten an Entwicklungspartnern oder InvestorInnen.

Auch wenn Hochschulen stärker Aktivitäten im Bereich der Motivierung und Identifikation von potentiellen Entrepreneuren setzen und in einzelnen Bereichen (bspw. IPR) auch beraten, sind sie nicht in der Position und Lage, Gründungen über einen längeren Zeit unabhängig und professionell zu begleiten.

Private Inkubatoren sind nicht für die Breite der Zielgruppe des AplusB-Programms offen, bieten aber einen guten Übergang für Fälle, die aus der Perspektive des AplusB-Programms nicht unterstützungsbedürftig sind (bspw. in der Entwicklung zu weit fortgeschritten sind).

- Wie präsentiert sich AplusB im europäischen/internationalen Vergleich? Welche Erfahrungen anderer Initiativen können für die Beurteilung und Weiterentwicklung von AplusB genutzt werden?

Die Betrachtung vergleichbarer Inkubatoren (Programme) mit dem AplusB-Programm zeigt, dass das Programm in Hinblick auf seine Ausrichtung und auch die Verankerung in der Politiklandschaft durchaus vergleichbar mit anderen Programmen ist und sich mit diesen auf einem sehr hohen Niveau messen kann. Mit der angestrebten Verankerung in den regionalen Innovations- und Politiksystemen sind die AplusB-Zentren auch kleiner strukturiert als der Durchschnitt der Inkubatoren in Deutschland und weisen eine höhere Betreuungsdichte auf als diese.

- Welche Kommunikations- und Vernetzungsstrukturen konnten seit Bestehen aufgebaut werden bzw. existieren aktuell? Welche Systematik liegt dem zu Grunde?

Das AplusB-Programm forciert den Austausch und die Positionierung auf unterschiedlichen Ebenen. Auf der nationalen Ebene sind es der Verein „Österreichisches Inkubatorennetzwerk AplusB“ und die AplusB Plattform, auf der regionalen Ebene laufende Abstimmungen und Positionierung im regionalen Unterstützungs- und Stakeholder-Umfeld.

- Die vom Verein „Österreichisches Inkubatorennetzwerk AplusB“ betreute Plattform ist zeitgemäß und rückt neben den Leistungen von Programm und Zentren vor allem die Gründungen selbst in den Vordergrund. Der Verein und seine Internetpräsenz erlauben eine stärkere Positionierung von AplusB als Qualitätssiegel und Auszeichnung. Teilweise sind Beschreibungen bereits in Englisch vorhanden. Mit Stand September 2015 gelang noch keine konsistente Präsenz in englischer Sprache, die sich einem breiteren internationalen Publikum öffnet.

- Der in der jüngeren Zeit noch zusätzlich verstärkte Austausch und Wissenstransfer im Rahmen der AplusB-Plattform und die damit forcierte konzeptionelle Abstimmung zwischen den Zentren, aber auch anlassbezogen mit involvierten Stakeholdern (regional, aws etc.) ist sehr positiv zu bewerten. Dies spiegeln auch die durchgeführten Gespräche wider. Die Plattform unterstützt den Austausch zwischen den Zentren und den einzelnen Beratern und organisiert ihre Treffen fach- und lösungsorientiert. Gleichzeitig ist festzustellen, dass Entwicklungen nicht zwangsläufig in die verbindliche Festlegung auf gemeinsame Standards oder „Good Practices“ münden müssen. Hier könnte die Plattform in Abstimmung mit dem Programmmanagement eine noch stärkere Rolle spielen.

- Der direkte Austausch zwischen den Gründungen einzelner Zentren sowie die Wahrnehmung gemeinsamer Angebote spielen nach wie vor eine untergeordnete Rolle und könnte initiiert und koordiniert über die Plattform noch ausgebaut werden. 
- Wie und in welchen Umfang sind die Zentren in das regionale Innovationssystem eingebettet?

Die Fokusgruppen attestieren den AplusB-Zentren eine wichtige Rolle im heimischen Innovationssystem. Durch Ihre spezifische Ausrichtung leisten die Zentren einen wesentlichen Beitrag zur Positionierung Österreichs als Gründungsland. Nach Einschätzung der befragten InterviewpartnerInnen konnten sich die Zentren, nach einigen Anlaufschwierigkeiten in den Anfangsjahren, gut in das jeweilige regionale Innovationssystem integrieren und Ihre Rolle und Funktion laufend anpassen und verbessern. Nach mittlerweile fast 13-jähriger Erfahrung in Inkubationsprozessen verfügen die Zentren heute über eine hohe Reputation und Sichtbarkeit im Innovationssystem, das auf einem gewachsenen Netzwerk aus Wissenschaft, Wirtschaft und Start-up Community fußt.

Die enge Anbindung an Universitäten und Fachhochschulen ist ein klares Alleinstellungsmerkmal der AplusB-Zentren. An Standorten mit Entrepreneurship-Lehrstühlen wird die Kooperation als besonders fruchtbar erachtet. Lt. Fokusgruppen sind die Schnittstellen zu Standortagenturen und anderen Inkubatoren ebenfalls von Bedeutung, wobei Synergiepotenziale im Awareness- und Pre-Inkubationbereich jedoch noch nicht ausreichend ausgeschöpft werden. Da sich das Angebot an Gründungsförderung und -unterstützung dynamisch weiterentwickelt, werden die Zentren künftig ihre Rolle stärker betonen und ihr Leistungsportfolio unter Berücksichtigung des regionalen Systems weiter optimieren müssen.

- Welche Erwartungshaltungen für die Zukunft haben die wichtigsten Stakeholder, weitere involvierte Universitäten, Fachhochschulen und zuständigen Landesdienststellen?

In nahezu allen Interviews mit Trägerorganisationen sowie Netzwerk- und Kooperationspartnern sowie in den Fokusgruppen wurde die strategische Wichtigkeit und hohe Relevanz von akademischen Unternehmensgründungen betont, die im Großen und Ganzen gute Zusammenarbeit mit den Zentren unterstrichen sowie den MitarbeiterInnen eine hohe Professionalität und Kompetenz der MitarbeiterInnen konstatiert. Die längerfristige, kontinuierliche Betreuung von Gründungsprojekten durch die AplusBZentren wird in den Fokusgruppen als einzigartig aufgefasst, die in dieser Form von keinem anderen Angebot oder Initiative geleistet wird. Der Grundtenor ist, dass die Arbeit im Programm weitergeführt werden soll.

Anzustreben ist künftig jedoch eine laufende Kommunikation und bessere Abstimmung zwischen AplusB und den regionalen Bedarfen und Politik, ähnlich wie es im ebenfalls von der FFG abgewickelten COMET Programm der Fall ist. Dabei sollen lt. Fokusgruppen die Synergien zu Träger- und Förderorganisationen, sowie ggf. weiteren regionalen Akteuren, bei der Definition der Leistungen künftig mitberücksichtigt und die Länderstellen, welche sich als zu wenig informiert und eingebunden sehen, auf Programmebene stärker miteinbezogen werden. Ähnlich wie bei COMET würde sich hier die Möglichkeit von Jahresgesprächen oder eine vergleichbare Kommunikationsplattform anbieten. 


\section{Struktur und Charakteristika der AplusB-Gründungen}

Im Folgenden werden Struktur und einige Charakteristika der AplusB-Gründungen auf der Grundlage der Daten des AplusB-Gründungs-Monitorings dargestellt.

\subsection{Ergebnisse des Gründungs-Monitoring}

Seit Beginn im Jahr 2002 wird das AplusB-Programm von einem Monitoring begleitet, das Informationen zu realisierten Gründungsprojekten mittels Online-Fragebögen dokumentiert. Die Befragung der GründerInnen erfolgte: beim Eintritt in das Zentrum (I), bei der Gründung (II), beim Austritt aus dem Zentrum (III) und in den ersten vier Geschäftsjahren nach der Gründung (IV).

Zwischen Dezember 2002 und Februar 2015 wurden in Summe 755 betreute Unternehmen (PreInkubation und Inkubation) erfasst. Von den in der Gründung involvierten 1.186 Personen wurden insgesamt 4.656 Fragebögen ausgefüllt, die die Basis für die weitere Analyse darstellen. Anhand der erfassten Fragebögen lässt sich die Frequentierung der AplusB-Zentren gut nachvollziehen. Der Rücklauf weitgehend vollständig ausgefüllter Eintrittsfragebögen (I) liegt bei annähernd $100 \%$. Grund hierfür ist, dass das Ausfüllen zumindest des Eintrittsfragebogens Bedingung für den Erhalt der Förderung ist. Die Rücklaufquote der weiteren Fragebögen (II, III, IV) ist etwas geringer.

Die Auswertungen des Gründungs-Monitoring fokussieren auf die Entwicklung der Anzahl und Art der Unternehmensgründungen sowie die Motive und Hemmnisse für die Gründung und Entwicklung eines Unternehmens. Weiters werden die Wirtschaftszweige und Branchen, in denen die gegründeten Unternehmen tätig sind, die Forschungs- und Entwicklungsintensität, die Qualifikation der MitarbeiterInnen sowie die Nutzung von Patenten dargestellt und analysiert. Im Anschluss wird die Finanzierungsstruktur in den ersten vier Geschäftsjahren und die Rolle von Venture-Capital- und BusinessAngel-Finanzierung beschrieben und diskutiert.

Das AplusB-Gründungs-Monitoring sticht mit seiner abgestimmten und mit Nachdruck verfolgten Datenfassung im Bereich der Programmförderung positiv hervor. Die folgenden Betrachtungen basieren im Wesentlichen auf den Erhebungen des Gründungs-Monitorings.

\section{Abbildung 6 Gesamtzahl der bisher im Rahmen des Gründungs-Monitorings erfassten Fragebögen}

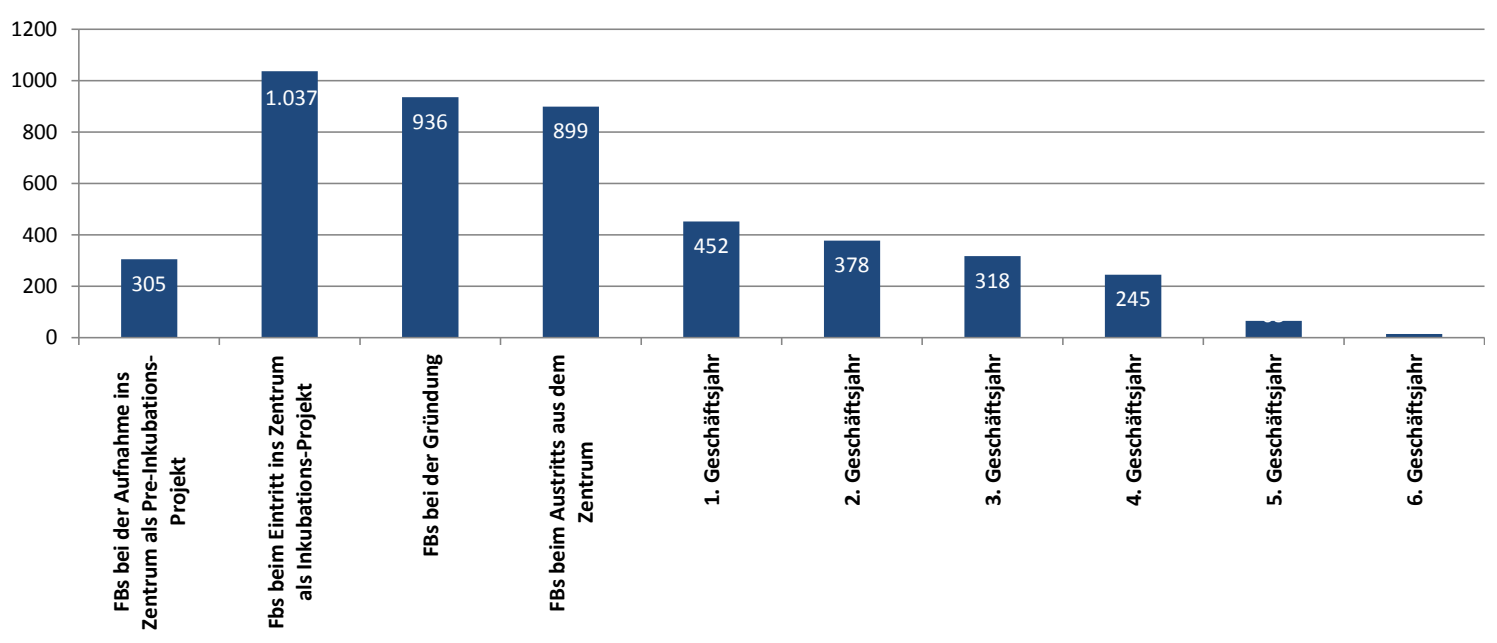

Quelle: AplusB-Gründungs-Monitoring, JR-Berechnungen

Von Dezember 2002 bis Februar 2015 konnten insgesamt 645 Gründungsvorhaben in den Zentren erfasst werden (Inkubation). Auch wenn die überwiegende Mehrheit der befragten Personen $(85 \%$ 
aller erfassten GründerInnen) im Team gegründet hat, sind nur rd. $60 \%$ (383) der erfassten Gründungsvorhaben Teamgründungen. Teamgründungen schließen im Durchschnitt 2,6 GründerInnen ein. In nur acht Ausnahmefällen handelte es sich um eine Gründungen mit Beteiligung durch ein anderes Unternehmen.

\subsubsection{Aufnahme und Realisierung von Gründungsvorhaben}

Abbildung 7 zeigt (periodenbereinigt) die durchschnittliche Zahl der Gründungsvorhaben. Dabei werden Teamgründungen jeweils als ein Gründungsfall gezählt. Das AplusB-Zentrum INITS in der Region Wien weist im Durchschnitt der Jahre den höchsten Anteil an Neuzugängen auf. Die beiden AplusBZentren in der Steiermark (SPG, ZAT) kommen in Summe auf die gleiche Zahl an neu aufgenommenen Gründungsvorhaben pro Jahr.

\section{Abbildung 7 Aufgenommene Gründungsvorhaben in den Jahren 2002-2015, absolut}

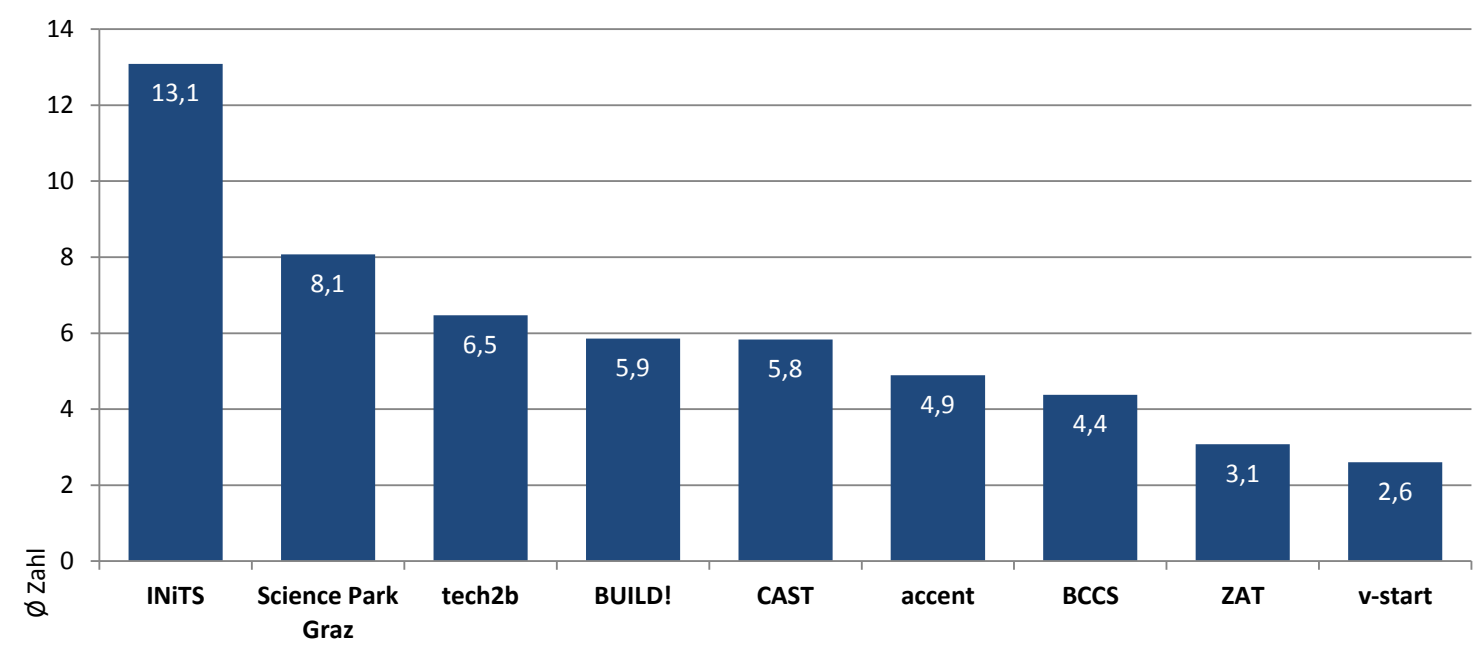

Quelle: AplusB-Gründungs-Monitoring, JR-Berechnungen

Da nur ein Teil der potentiellen bzw. interessierten Gründungsvorhaben in die AplusB-Zentren aufgenommen werden kann, übersteigt die Zahl der Gründungsvorhaben, mit denen die AplusB-Zentren in der Präinkubationsphase befasst sind, die Zahl der dargestellten Eintritte in die AplusB-Zentren bei Weitem. Dabei ist festzustellen, dass lediglich $9 \%$ der im Rahmen des Gründungs-Monitorings ersterfassten GründerInnen Frauen sind. Dieser Anteil liegt deutlich unter dem durchschnittlichen Anteil an Frauen an technischen Universitäten und außeruniversitären Forschungseinrichtungen (siehe dazu auch Abschnitt 4.2.2.).

Die folgende Darstellung zeigt die im Zeitverlauf bei den jeweiligen AplusB-Zentren angelegten Datensätze (Abbildung 8). Dabei sind durchaus beachtliche Schwankungen nachvollziehbar. Im Fall der AplusB-Zentren INITS, Science Park Graz oder auch Tech2B ist nach einem kurzen Rückgang zum Zeitpunkt des Ausbruch der Wirtschafts- und Finanzkrise ein deutlicher Anstieg der neu aufgenommenen Gründungsvorhaben festzustellen. 
Abbildung 8 Entwicklung der in den einzelnen AplusB-Zentren aufgenommenen Gründungsvorhaben, gesamt seit 2002

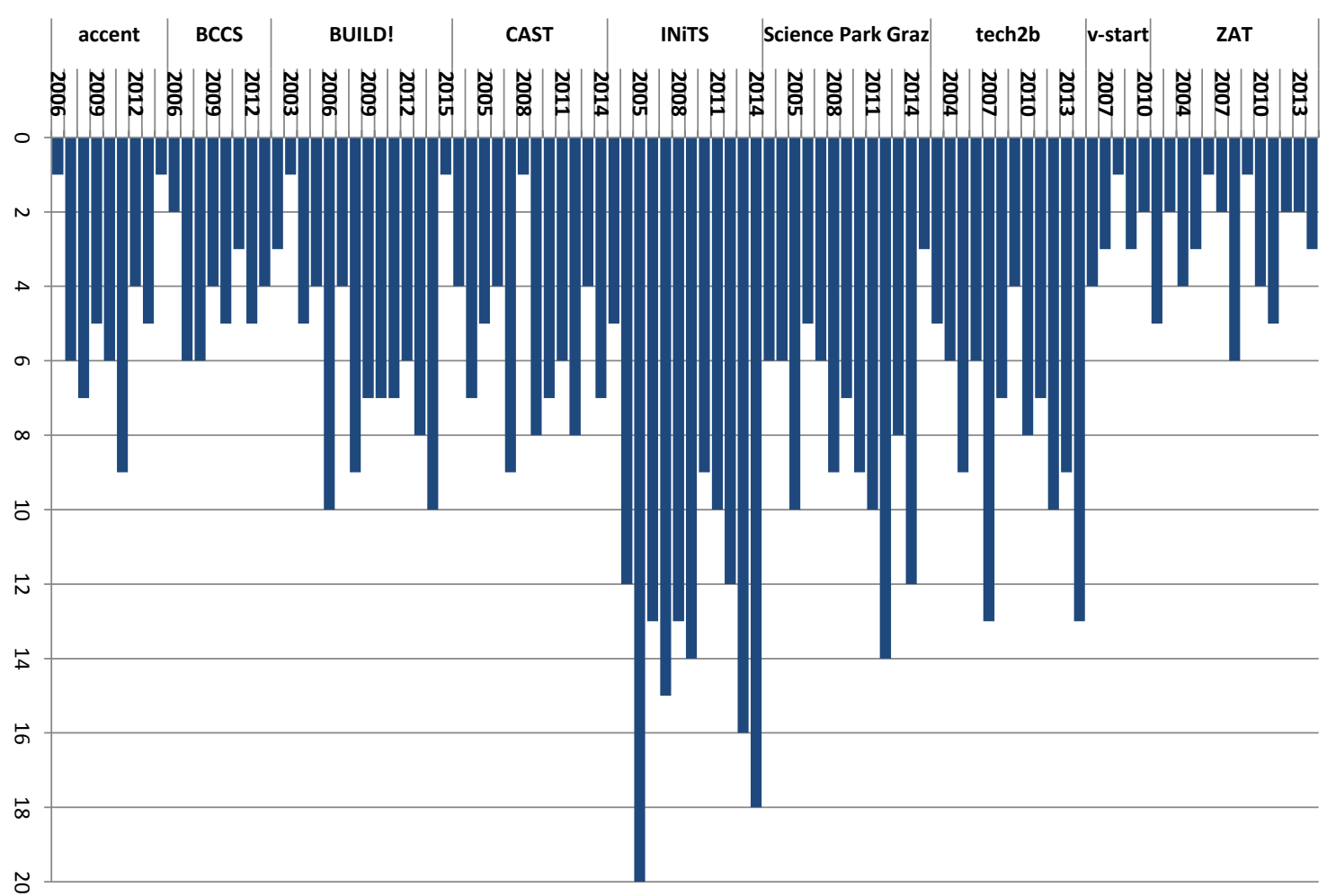

Quelle: AplusB-Gründungs-Monitoring, JR-Berechnungen

Abbildung 9 stellt die seit 2002 in den AplusB-Zentren aufgenommenen Gründungsvorhaben den im Laufe der Zeit erfolgreich gegründeten bzw. ausgetretenen Projekten ${ }^{32}$ gegenüber. Erwartungsgemäß zeigt sich dabei eine zu Beginn stark wachsende Anzahl an Gründungsvorhaben und -projekten, welche sich ab etwa 2008 auf einem Niveau von ca. 60 Gründungen pro Jahr in allen AplusB-Zentren gesamt einpendelte. 2011 sank die Entwicklung auf ca. 50 Gründungen pro Jahr ab, blieb im weiteren Verlauf jedoch in etwa stabil. Basis hierfür sind lt. Eigenrecherche der Zentren pro Jahr rd. 800 evaluierte Ideen, die in Summe zu ca. 500 Erstgesprächen führen.

Zwischen Dezember 2002 und Februar 2015 wurden insgesamt 645 Gründungsvorhaben in AplusBZentren aufgenommen (Inkubation). 568 Unternehmen schafften den Schritt zur formalen Gründung, 539 sind ausgetreten. Anzumerken ist, dass die Reihenfolge im Gründungsprozess, also ob die Gründung nach dem Eintritt oder nach dem Austritt aus dem Zentrum erfolgt, erheblich variiert. ${ }^{33}$

32 Im Rahmen der AplusB-Community werden unterschiedliche Begrifflichkeiten für die noch realisierten Gründungen eingesetzt.

${ }^{33}$ Es ist an zu dieser Stelle anzumerken, dass nicht alle Unternehmen, die angegeben haben, die Gründung formal umzusetzen auch ein Jahr danach von der Statistik noch erfasst werden konnten. 
Abbildung 9 Entwicklung der Gründungsvorhaben und -projekte, gesamt, 2002-2015

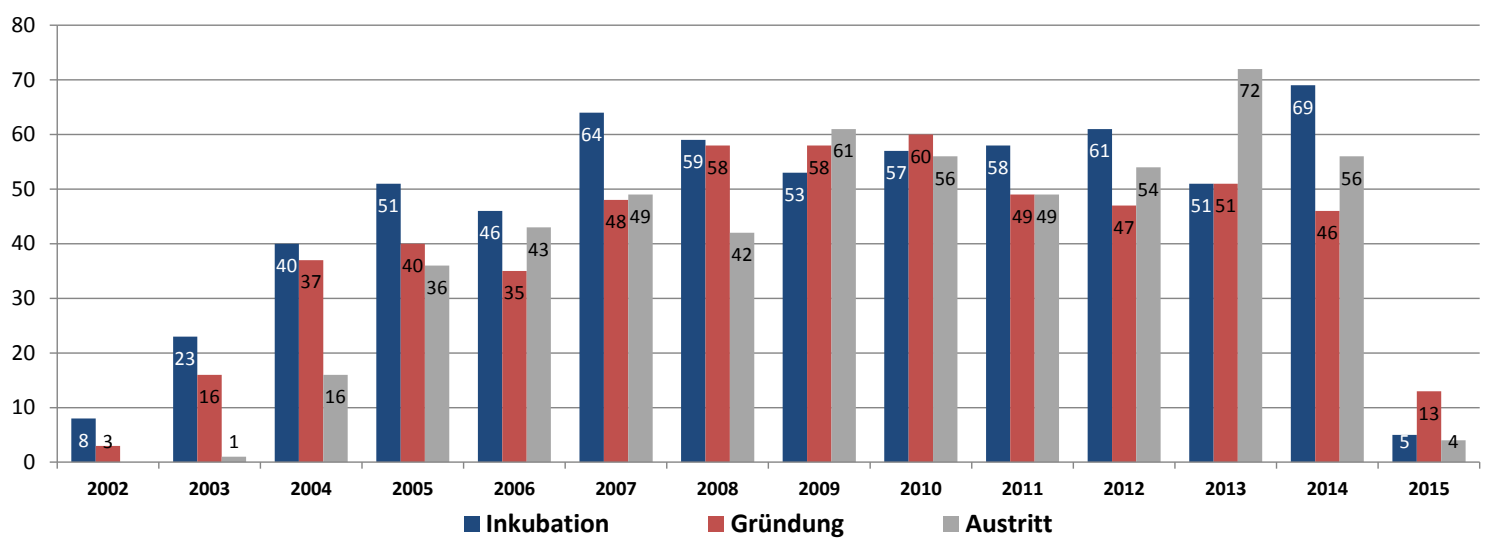

Quelle: AplusB-Gründungs-Monitoring, JR-Berechnungen

Der überwiegende Anteil (57\%) der erfolgreich gegründeten AplusB-Unternehmen wählt die Gesellschaftsform einer $\mathrm{GmbH}$, gefolgt von Einzelunternehmen (18,2\%).

In fast $90 \%$ der erfassten Fälle erfolgte der Austritt aus dem AplusB-Zentrum planmäßig nach der erfolgten Gründung. Nicht in allen Fällen gelang es allerdings, die Unternehmensgründung formal vor dem Austritt aus dem AplusB-Zentrum zu realisieren. In $2 \%$ der erfassten Fälle wurde das Gründungsprojekt eingestellt. Weitere $9 \%$ der betreuten Fälle gaben an, die Gründung nach dem Austritt aus dem AplusB-Zentrum durchführen zu wollen. Die verbleibenden $2 \%$ konnten sich nicht festlegen, ob nach dem Austritt noch eine Gründung erfolgen wird.

Abbildung 10 Anteil der Gründungsvorhaben, die planmäßig die Inkubationsphase abgeschlossen haben (in \%)

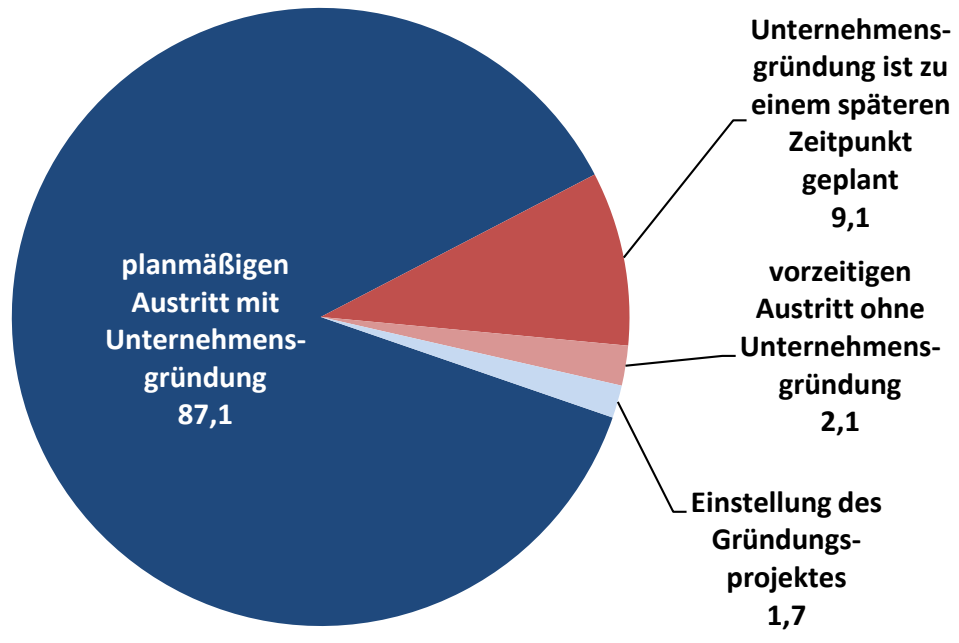

Quelle: AplusB-Gründungs-Monitoring, JR-Berechnungen

Der überwiegende Anteil der erfolgreich gegründeten AplusB-Unternehmen wählt die Gesellschaftsform einer $\mathrm{GmbH}$. 
Abbildung 11 Verteilung nach Gesellschaftsformen der AplusB-Gründungen (in \%)

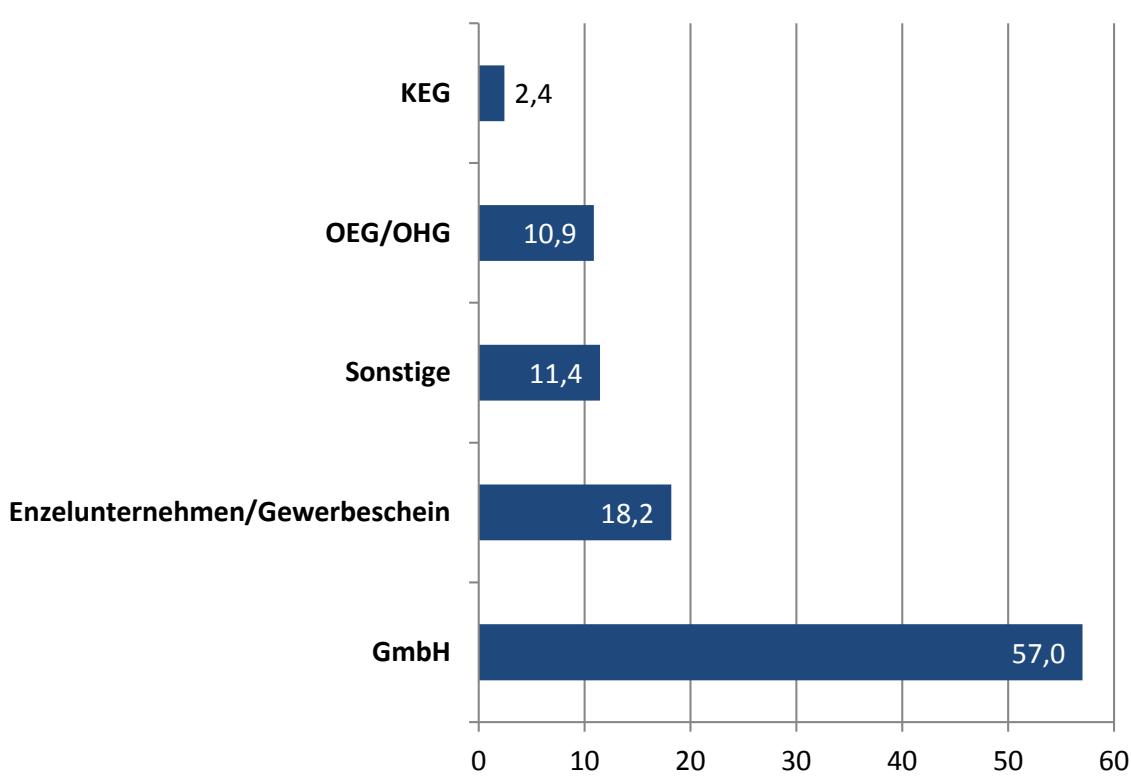

Quelle: AplusB-Gründungs-Monitoring, JR-Berechnungen

\subsubsection{Strukturmerkmale der AplusB-Gründungsvorhaben}

Im Rahmen der Erhebungen des AplusB-Gründungs-Monitorings wird das mit den Gründungsvorhaben verknüpfte Technologiefeld erfasst, allerdings nicht die Branche (wirtschaftliche Aktivität entsprechend der NACE-Klassifikation). Die zuverlässigste Quelle, die die Branche eines Unternehmens festhält, stellt das Unternehmensregister der Statistik Austria dar. Für jene AplusB-Gründungsvorhaben, die zum Zeitpunkt der Abfrage bereits formal gegründet waren und die bereits an einer offiziellen Erhebung teilgenommen haben, ist auch eine Zuordnung zur Branche möglich (siehe Abbildung 12). ${ }^{34}$

34 Die Unternehmensstatistik erfasst nur realisierte Gründungsvorhaben, die mehr als ein halbes Jahr Bestand haben. Die relativ gute Verfügbarkeit von Kontakt- und Identifikationsdaten im Rahmen des AplusB-Programms war ausschlaggebend dafür, dass ein Großteil der AplusB-Gründungen auch im Unternehmensregister der Statistik Austria identifiziert werden konnte. 
Abbildung 12 Branchenstruktur der in der offiziellen Statistik erfassten AplusB-Gründungen (in \%)

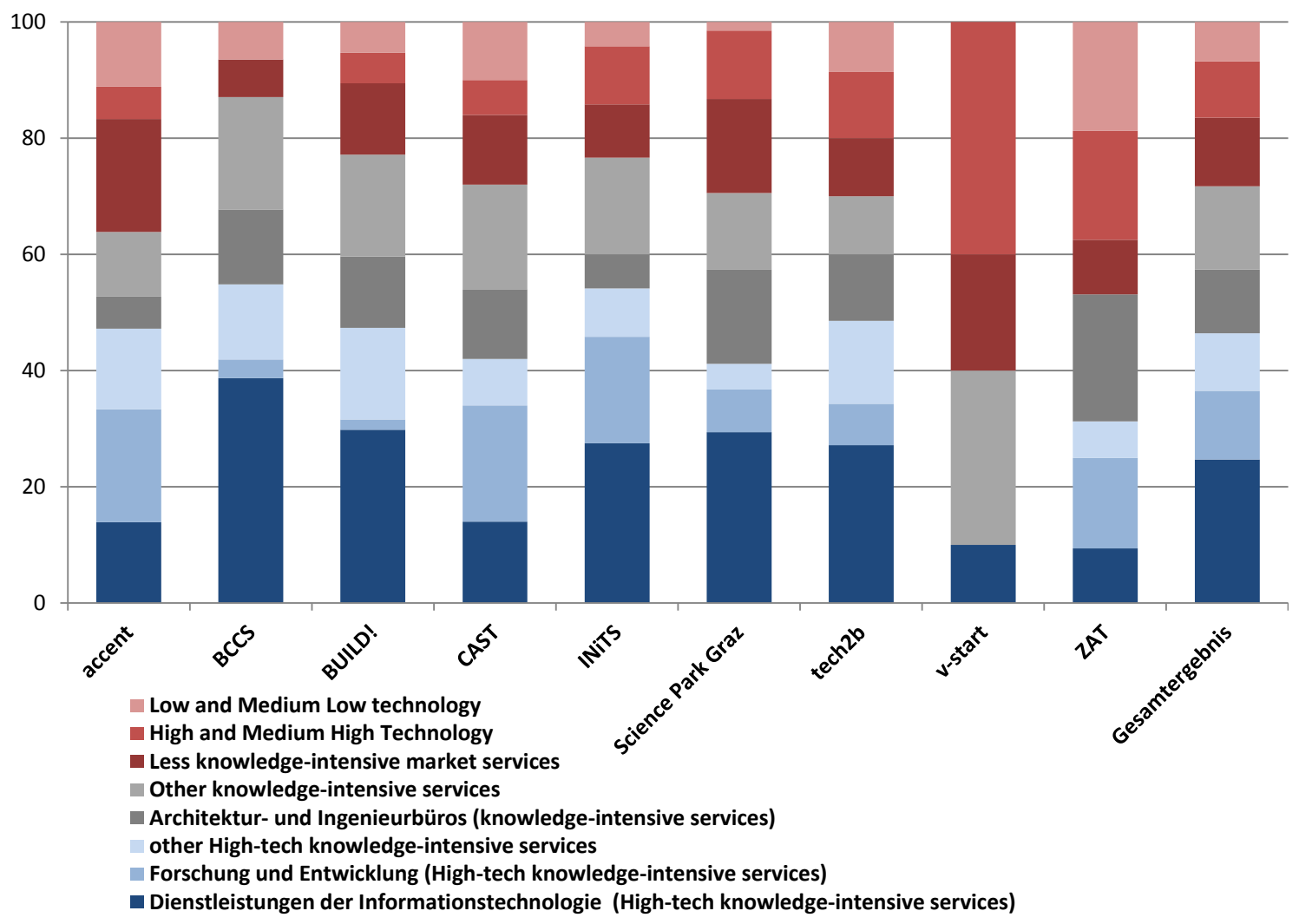

Quelle: AplusB-Gründungs-Monitoring, Statistik Austria Unternehmensregister, JR Berechnungen

$84 \%$ der erfassten AplusB-Gründungen sind dem Dienstleistungsbereich zuzuordnen. Dies ist nicht ungewöhnlich und auch nachvollziehbar. Innovative Gründungen arbeiten in den ersten Jahren als Forschungs- oder Beratungsdienstleister bzw. handeln mit komplementären Produkten.

Die Herstellung von Waren setzt entsprechendes Kapital sowie in weiterer Folge die Etablierung von Vertriebskanälen voraus.

Knapp die Hälfte (46\%) der beobachtbaren AplusB-Gründungen ist dem Bereich der wissensintensiven Hochtechnologiedienstleistungen (dominiert von IT-Dienstleistungen) zuzuordnen. Die geringfügigen Unterschiede in der Branchenstruktur der von den einzelnen AplusB-Zentren betreuten Gründungen erklären sich durch regionale Schwerpunkte der vorliegenden Unternehmens- und Forschungslandschaft.

Verknüpft man die identifizierte Branchenzugehörigkeit der AplusB-Gründungen mit den im Rahmen des AplusB-Gründungs-Monitorings erfassten Technologiebereichen der Gründungsvorhaben, so wird deutlich, dass rd. die Hälfte der AplusB-Gründungen wesentlich von Informationstechnologien geprägt werden.

Die identifizierten AplusB-Gründungen, die F\&E als primäre Aktivität angeben, sind zu einem wesentlichen Teil dem Life Science Bereich zuzuordnen. Die Unternehmensentwicklung ist hier anders zu beurteilen als in anderen Bereichen. 
Abbildung 13 Struktur der im Rahmen des Gründungs-Monitorings registrierten Ersterfassungen, nach Branchen und Technologiefeldern (in \%)

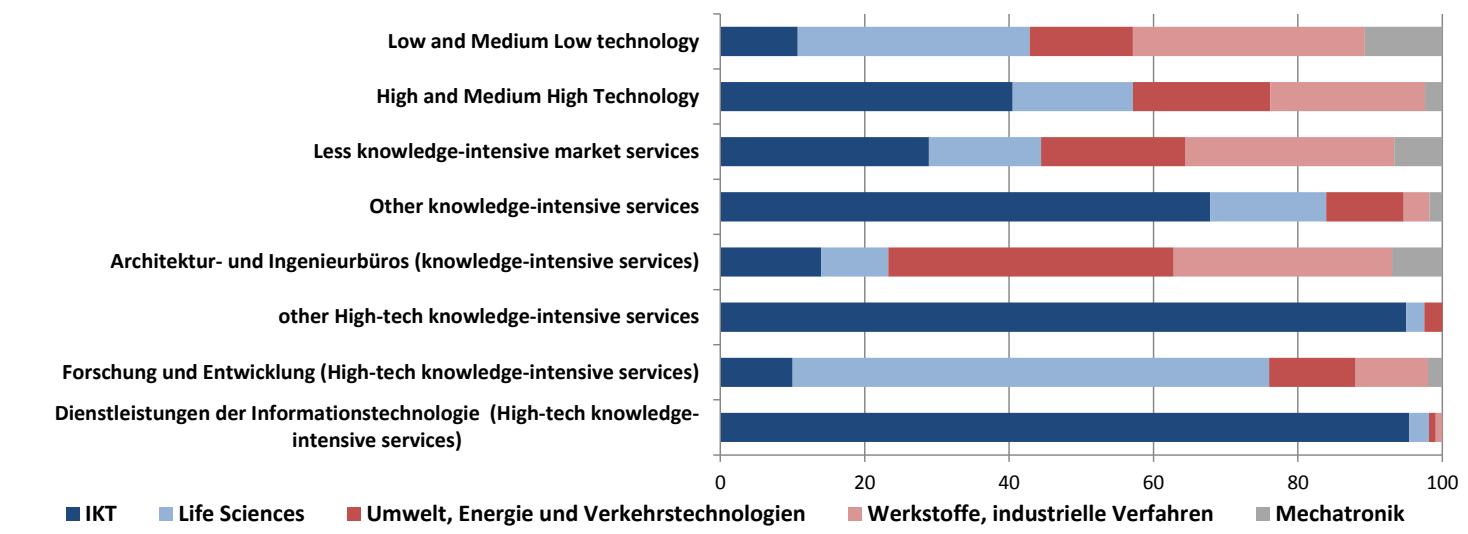

Quelle: AplusB-Gründungs-Monitoring, Statistik Austria Unternehmensregister, JR-Berechnungen

Junge Unternehmen im Bereich der Life Science bleiben häufig für immer „F\&E-Dienstleister“. Aufgrund des hohen Investitionsaufwandes im Rahmen notwendiger klinischer Tests und Zulassungsverfahren realisiert sich das vorhandene Wachstumspotential häufig erst nach der Übernahme durch ein größeres finanzkräftiges Unternehmen.

Es kündigt sich an, dass auch viele AplusB-Gründungen, die nicht dem Branchenbereich F\&E zuzuordnen sind, nach der Gründung F\&E-aktiv bleiben. Bei Angaben, die die Phase nach der Gründung betreffen, stellt sich heraus, dass das Gründungs-Monitoring ein verzerrtes Bild zeigt, da die Antwortbereitschaft mit jedem Jahr rapide sinkt. Wie die Ergebnisse der Befragung von AplusB-Gründungen zeigen, betreiben rd. $80 \%$ der erfolgreichen AplusB-Gründungen in den ersten Jahren weiterhin kontinuierlich F\&E. Aufbauend auf einer direkten Verknüpfung mit dem Unternehmensregister und der F\&E-Erhebung der Statistik Austria wird im Abschnitt 5.2.1 genauer darauf eingegangenen, wie hoch der Anteil der AplusB-Unternehmen ist, der auch noch nach der erfolgten Gründung F\&E (entsprechend der Definition des Frascati-Manuals) durchführt.

\subsubsection{Hintergründe der Entscheidung für eine Gründung im AplusB-Zentrum}

Das AplusB-Programm setzt sich neben der adäquaten Begleitung und Betreuung von innovativen Gründungen konkrete Ziele im Bereich von Bewusstseinsbildung und Mobilisierung bzw. Identifikation potentieller GründerInnen. 
Abbildung 14 Persönliche Motive der potentiellen AplusB-GründerInnen (in \%)

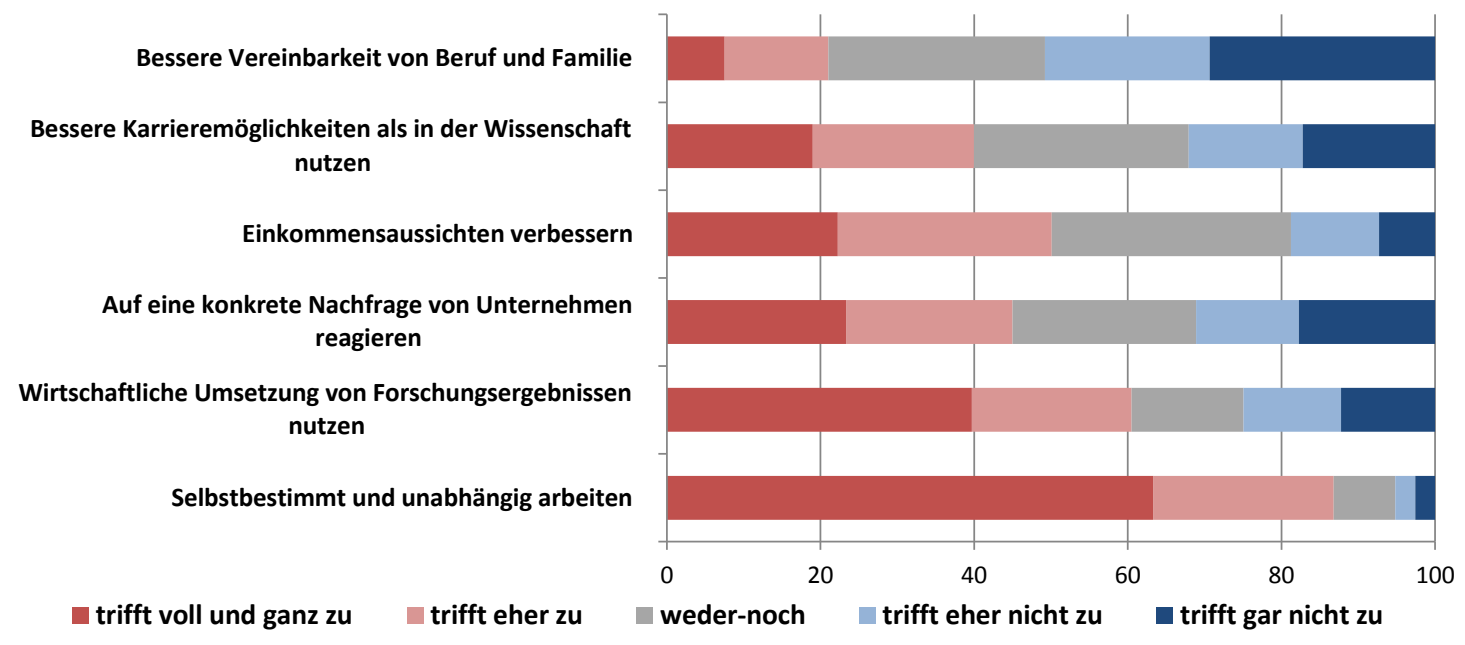

Mehrfachantworten möglich

Quelle: AplusB-Gründungs-Monitoring, JR-Berechnungen

Die (potentiellen) AplusB-GründerInnen wurden demnach auch nach den persönlichen Motiven gefragt, die sie zur Gründung bewegen. An erster Stelle wurde die Möglichkeit, selbstständig und unabhängig zu arbeiten, genannt. Andere persönliche Motive, wie beispielsweise die Verbesserung der Einkommens- oder Karriereaussichten, spielen eine deutlich geringere Rolle. Auch die Verbesserung der Vereinbarkeit von Beruf und Familie spielt eine geringe Rolle unter den angegebenen Motiven.

Über $60 \%$ der GründerInnen unterstreichen die ,wirtschaftliche Umsetzung von Forschungsergebnissen" als Beweggrund. 45 \% geben eine konkrete Nachfrage von Unternehmen als Beweggrund an.

Die AplusB-GründerInnen wurden darüber hinaus gefragt, auf welchem Weg sie auf das AplusBZentrum bzw. sein Unterstützungsangebot aufmerksam wurden. Dabei stellte sich Mundpropaganda als der wichtigste Kommunikationskanal der AplusB-Zentren heraus (siehe Abbildung 15).

Abbildung 15 Bedeutung unterschiedlicher Wege, auf denen die GründerInnen vom AplusB-Zentrum bzw. Programm erfahren haben (in \%)

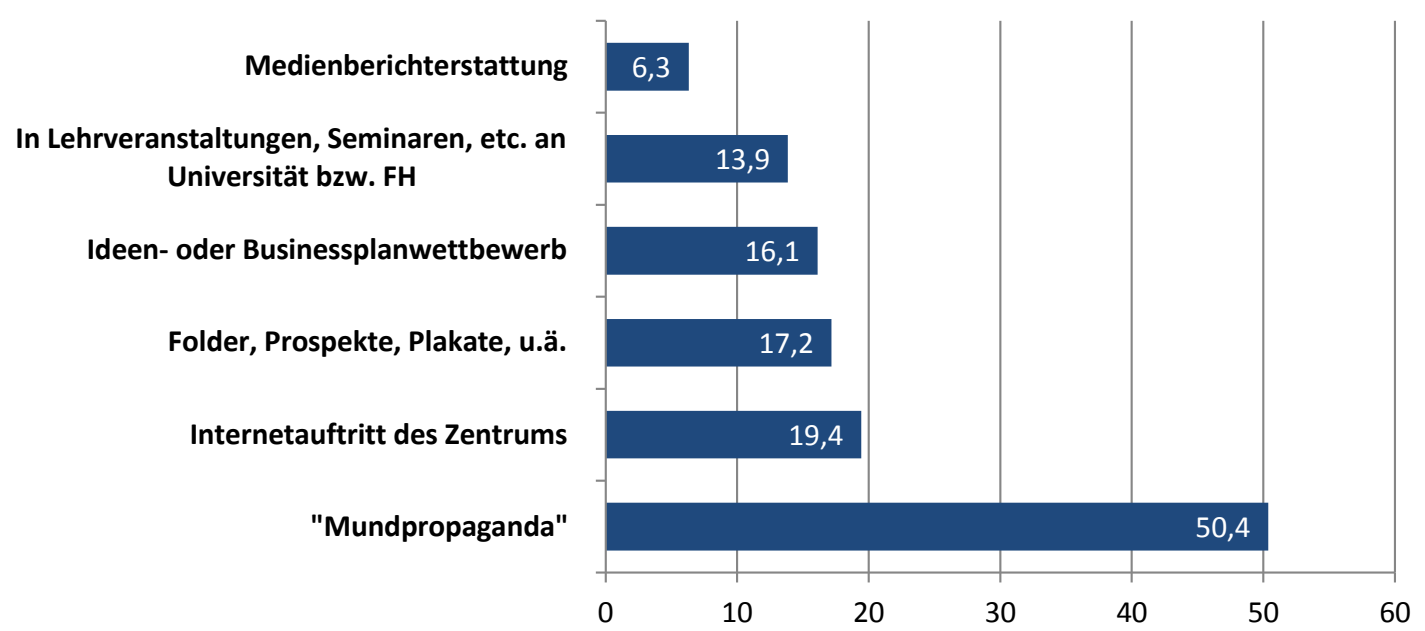

Mehrfachantworten möglich

Quelle: AplusB-Gründungs-Monitoring, JR-Berechnungen

Die einzelnen Zentren bedienen mögliche Kommunikationsinstrumente in unterschiedlicher Intensität, wie sich auch anhand der Unterschiede in der Beurteilung der in Abbildung 15 dargestellten Alternativen äußert. Die Mundpropaganda steht überall unangefochten an der ersten Stelle. Während beispiels- 
weise im Fall des AplusB-Zentrums CAST Ideen- und Businessplanwettbewerbe besonders hervorgestrichen werden, trifft dies im Fall anderer Zentren auf den Internetauftritt (INITS) oder auch Folder und Projekte (build!) zu.

Abbildung 16 Erwartungen der GründerInnen in Hinblick auf die Unterstützung im AplusB-Zentrum (in \%)

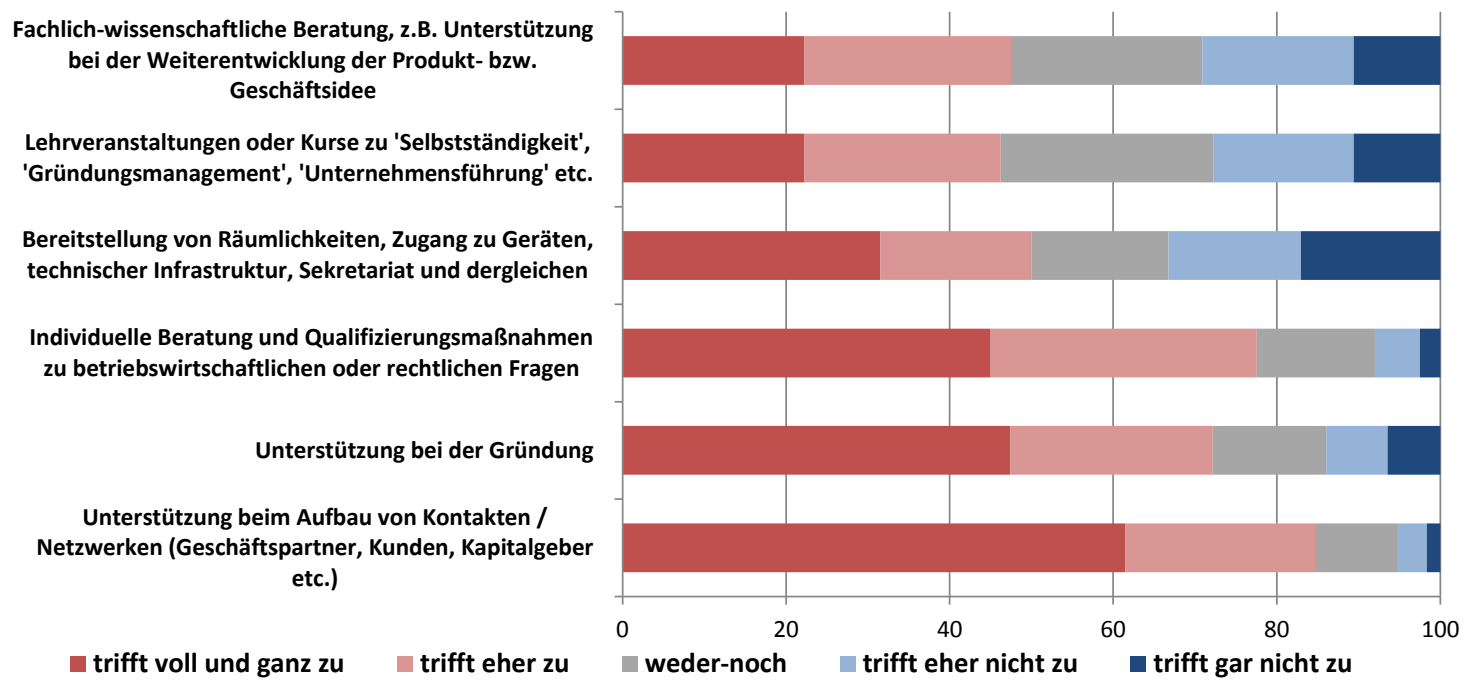

Quelle: AplusB-Gründungs-Monitoring, JR-Berechnungen

Die AplusB-GründerInnen wurden schließlich auch in Hinblick auf ihre Erwartungen, was die Unterstützung im AplusB-Zentrum anbelangt, befragt. Noch vor der individuellen Beratung und Unterstützung bei der formalen Gründung wurde dabei die Unterstützung beim Aufbau von Kontakten und Netzwerken (GeschäftspartnerInnen, KundInnen und KapitalgeberInnen) genannt. In deutlich geringerem Ausmaß erwarten sich die befragten GründerInnen Vorteile durch den Zugang zu Ausbildungen, durch fachlich-wissenschaftliche Beratung (bzw. Weiterentwicklung der Produkt- bzw. Geschäftsidee) oder die Bereitstellung von Infrastrukturen.

\subsubsection{Die Wissens- und Qualifikationsbasis der beobachteten AplusB-Gründungen}

Das AplusB-Programm unterstützt gezielt in Hinblick auf den Innovationsgrad und das zugrundeliegende Wissen anspruchsvolle Gründungsvorhaben. Auch wenn die Teilnahme am Programm für Nicht-AkademikerInnen nicht per se ausgeschlossen ist, ist das Programm nach wie vor vom Gedanken getragen, wissensintensive, akademische Gründungen voranzutreiben (siehe Abbildung 17).

Die AplusB-GründerInnen wurden dementsprechend befragt, welche der folgenden Faktoren für die Initiierung der Gründung unverzichtbar waren.

Mehr als die Hälfte (575) gab an, dass neue Forschungsergebnisse, an deren Erarbeitung die GründerInnen im Rahmen ihres Studiums oder ihrer wissenschaftlichen Tätigkeit mitgewirkt hatten, von groBer Bedeutung oder sogar unverzichtbar für die Initiierung der Gründung waren.

$68 \%$ maßen wissenschaftlichen Methoden oder Techniken, die an einer wissenschaftlichen Einrichtung erlernt wurden, große Bedeutung bei oder sahen diese für die Initiierung der Gründung als unverzichtbar an.

84 \% maßen besonderen Fähigkeiten, die sie im Rahmen ihres Studiums oder der Tätigkeit an wissenschaftlichen Einrichtungen erworben hatten, große Bedeutung bei oder bezeichneten diese sogar als unverzichtbar für die Initiierung der Gründung (45\%). 
Abbildung 17 Für die Initiierung und Gründung von AplusB-Fällen „,unverzichtbare“ Faktoren (in \%)

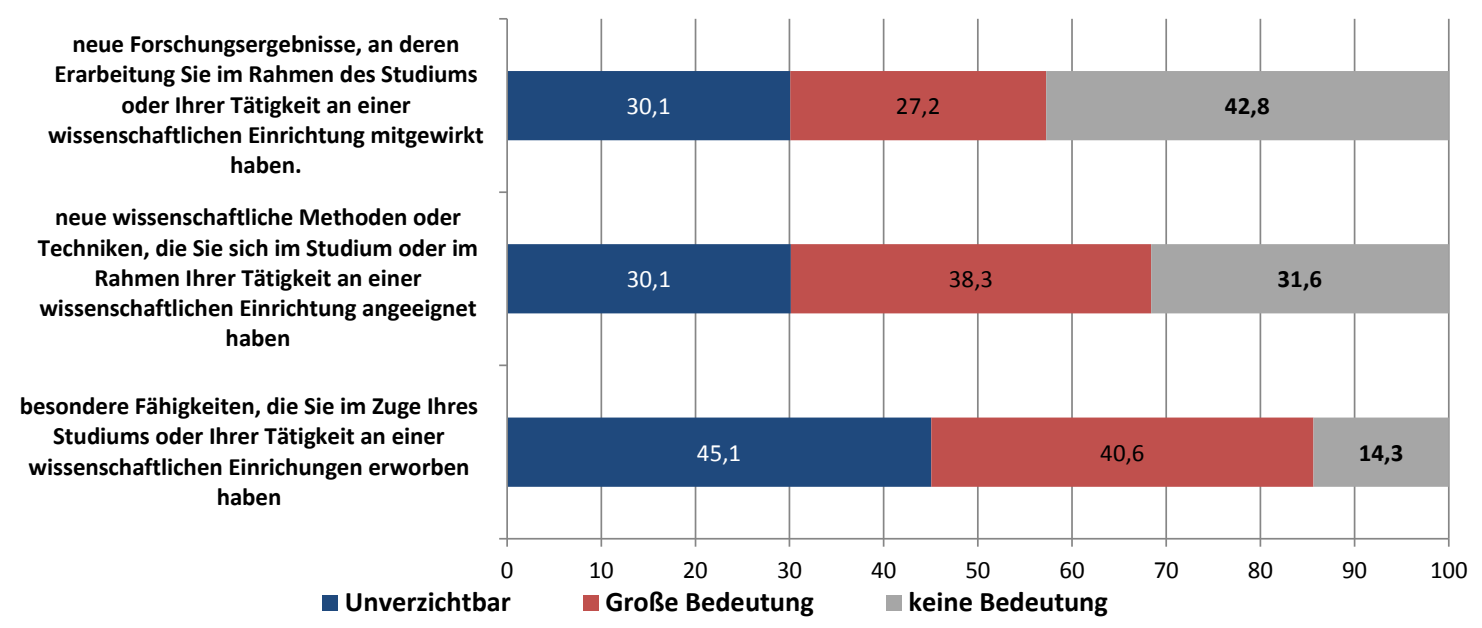

Quelle: AplusB-Gründungs-Monitoring, JR-Berechnungen

Wissenschaftliche Einrichtungen und die hier erworbenen Qualifikationen spielen für die AplusBGründerInnen eine erhebliche Rolle. Abbildung 18 zeigt, dass $76 \%$ der in den AplusB-Zentren aufgenommenen GründerInnen über eine Hochschulausbildung verfügen. $27 \%$ der AplusB-Gründungen weisen einen Doktorats-Abschluss auf.

Abbildung 18 Verteilung nach höchsten abgeschlossenen Ausbildungen der befragten AplusBGründerInnen (in \%)

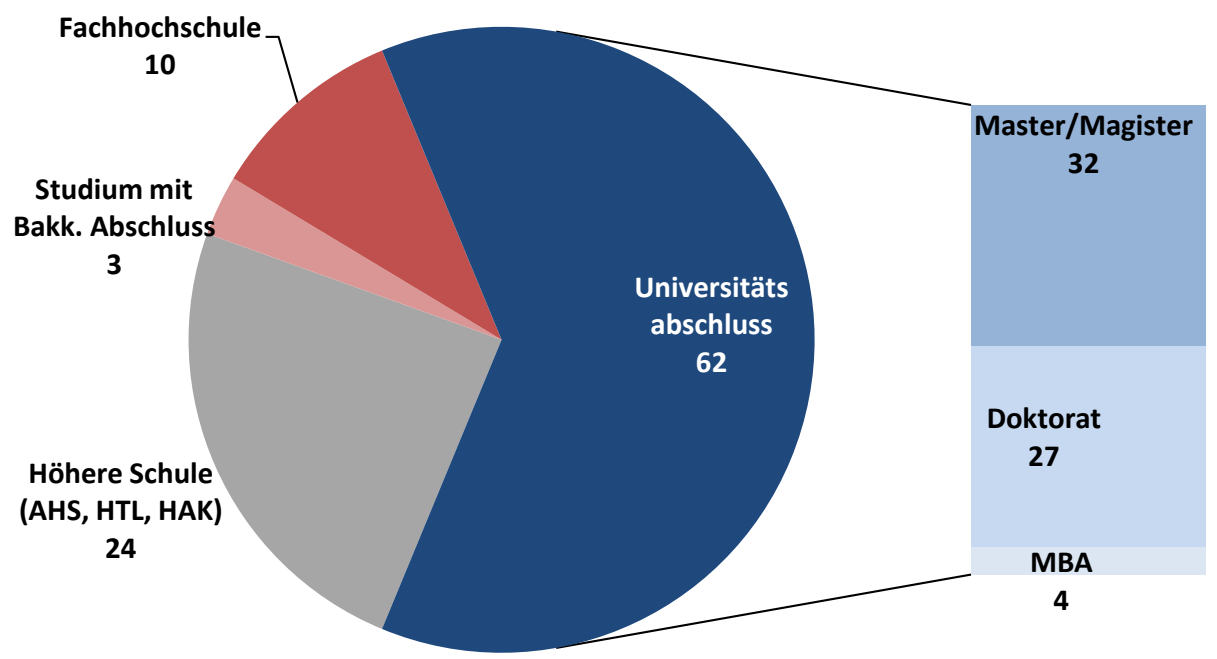

Quelle: AplusB-Gründungs-Monitoring, JR-Berechnungen

Wie Abbildung 19 zeigt, bestehen zwar Unterschiede hinsichtlich der Qualifikationsstruktur der in den einzelnen Zentren aufgenommenen AplusB-GründerInnen, diese stehen aber nicht zwangsläufig im Zusammenhang mit der Struktur des jeweiligen Hochschulstandortes. Auch in den AplusB-Zentren an den großen Hochschulstandorten Graz und Wien beträgt der Anteil der AplusB-GründerInnen ohne Hochschulabschluss rd. $20 \%$. Hier ist jedoch zu vermuten, dass sich unter den GründerInnen ein hoher 
Anteil von StudentInnen befindet, die ihren (akademischen) Ausbildungsweg noch nicht beendet haben und in der Erhebung ihren aktuell höchsten Bildungsabschluss angeben, etwa AHS, HTL oder HAK Matura.

Abbildung 19 Verteilung nach höchsten abgeschlossenen Ausbildungen der befragten AplusBGründerInnen in den einzelnen AplusB-Zentren (in \%)

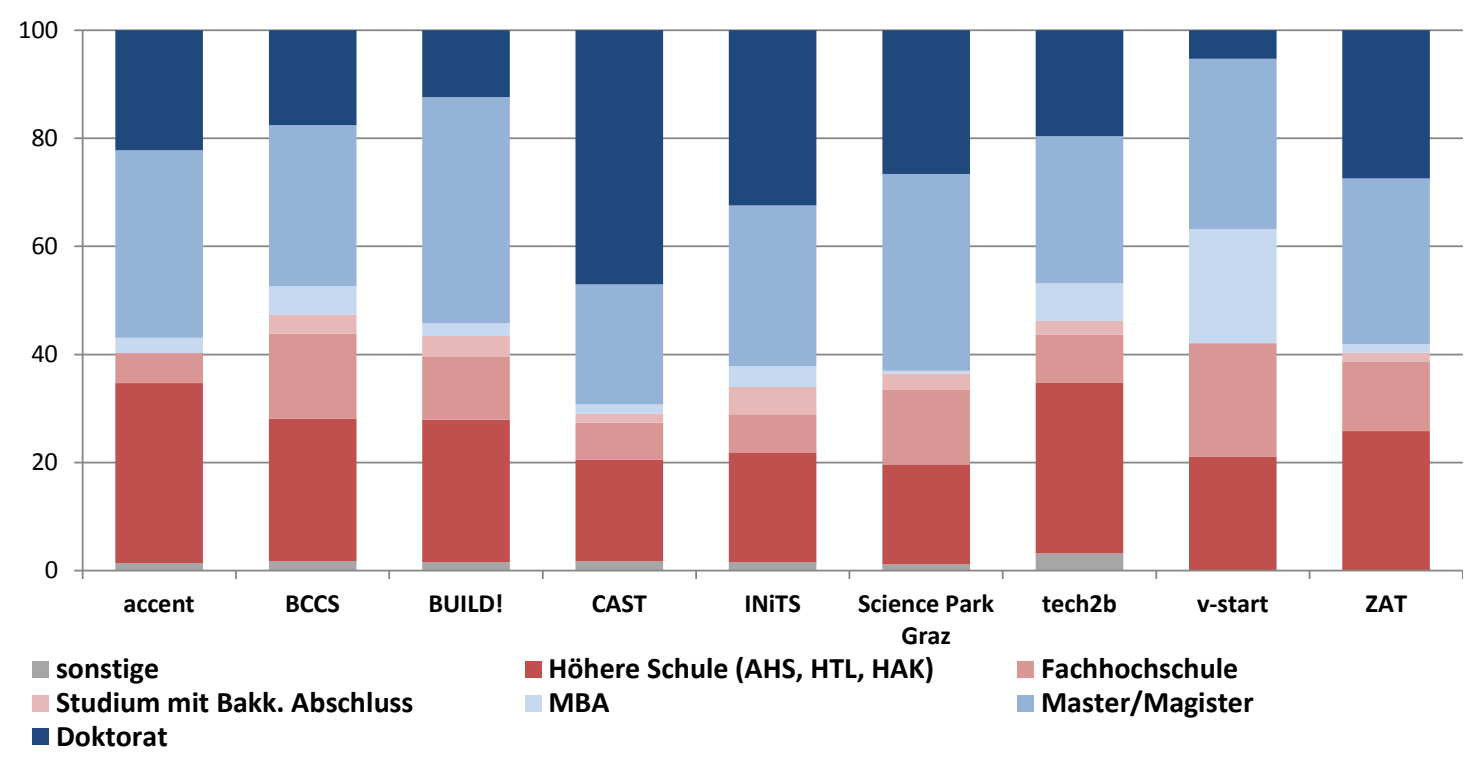

Quelle: AplusB-Gründungs-Monitoring, JR-Berechnungen

Wie anhand der im Rahmen des AplusB-Gründungs-Monitorings durchgeführten Befragung deutlich wird, konnten mehr als die Hälfte der AplusB-GründerInnen Kontakte aus dem wissenschaftlichen Bereich für ihr Gründungsvorhaben nutzen.

Darüber hinaus greifen AplusB-GründerInnen im erheblichen Ausmaß auf patentiertes Wissen zurück. Es ist dabei erwähnenswert, dass es sich dabei in der Mehrzahl der Fälle nicht um patentiertes Wissen handelt, das von den jeweiligen GründerInnen im Vorfeld im Rahmen der Tätigkeit in einer wissenschaftlichen Einrichtung selbst entwickelt wurde. 
Abbildung 20 Nutzung und Entwicklung von Patenten in den gegründeten Unternehmen, 2002-2013

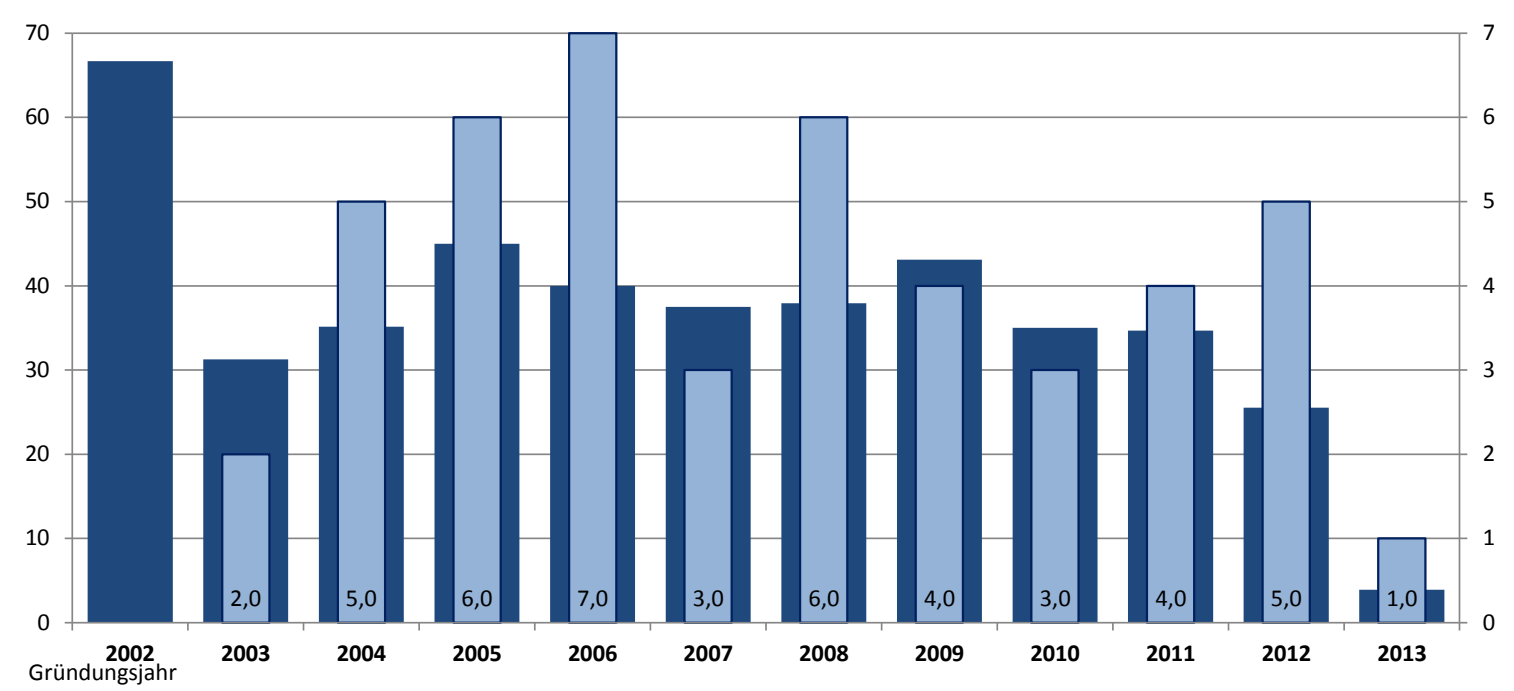

- Anteil der gegründeten Unternehmen, die in dem Kern-Geschäftsbereich im Laufe des letzten Jahres Eigen-oder Fremdpatente genutzt hatten

$\square$ Gesamtzahl der Patente der gegründeten Unternehmen, die von ForscherInnen im Umfeld von Unis, FHs oder RTOs entwickelt wurde

Quelle: AplusB-Gründungs-Monitoring, JR-Berechnungen

Unabhängig von der höchsten abgeschlossenen Ausbildung geben nahezu alle befragten GründerInnen (95\%) an, künftig mit wissenschaftlichen Einrichtungen (Universitäten, Fachhochschulen, Forschungseinrichtungen) kooperieren zu wollen. Dies gibt einen Hinweis der positiven Wirkung des Programms auf die langfristige Entwicklung von Kooperationen zwischen Wissenschaft und Wirtschaft.

\subsubsection{Herausforderungen und erste Erfahrungen der AplusB-GründerInnen}

Das AplusB-Programm verfolgt das Ziel, anspruchsvolle, innovative Gründungen mit einem entsprechenden Markt- und Wachstumspotential zu unterstützen. AplusB-GründerInnen sind im Zuge ihres Gründungsvorhabens mit sehr unterschiedlichen Herausforderungen konfrontiert (siehe Abbildung 21). Die drei von den AplusB-GründerInnen am stärksten hervorgehobenen Herausforderungen sind der Mangel an qualifiziertem Personal, der Mangel an geeigneten Finanzierungsquellen sowie die mangelnde Kenntnis von Markt und Kunden.

Aufgrund der Angaben der befragten GründerInnen lässt sich die Annahme, dass AplusB-Gründungen an den großen Forschungs- und Industriestandorten (Wien, Steiermark, Oberösterreich) in einem deutlich geringeren Ausmaß vom Mangel an qualifiziertem Personal betroffen sind, nicht bestätigen. 
Abbildung 21 Hemmnisse bzw. Herausforderungen, mit denen AplusB-GründerInnen konfrontiert sind (in \%)

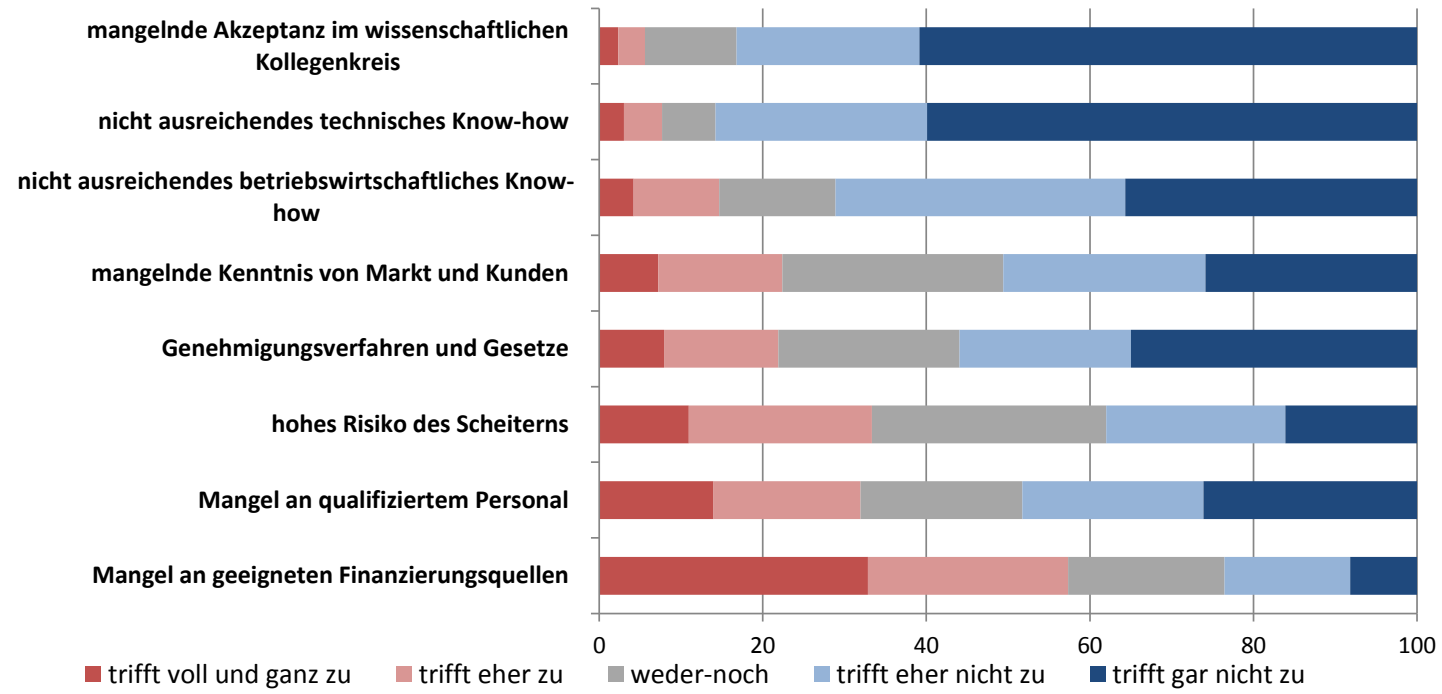

Quelle: AplusB-Gründungs-Monitoring, JR-Berechnungen

Wenig im Vordergrund stehen im Allgemeinen das Risiko zu scheitern, regulative Hemmnisse oder Qualifizierungslücken im technischen oder betriebswirtschaftlichen Bereich.

In Hinblick auf die eingesetzten Finanzierungsquellen wurden die AplusB-GründerInnen vertiefend befragt. In Abbildung 22 wird deutlich, dass es den AplusB-GründerInnen zum Zeitpunkt der Gründung gelingt, zusätzlich zu den eingesetzten Eigenmitteln einen Anteil von knapp $60 \%$ durch Außenfinanzierung abzudecken. An dieser Stelle ist allerdings anzumerken, dass sich die Bedingungen für eine private Außenfinanzierung mit dem Zeitpunkt der Gründung in der Regel verbessern.

\section{Abbildung 22 Finanzierungsquellen der AplusB-GründerInnen (in \%)}

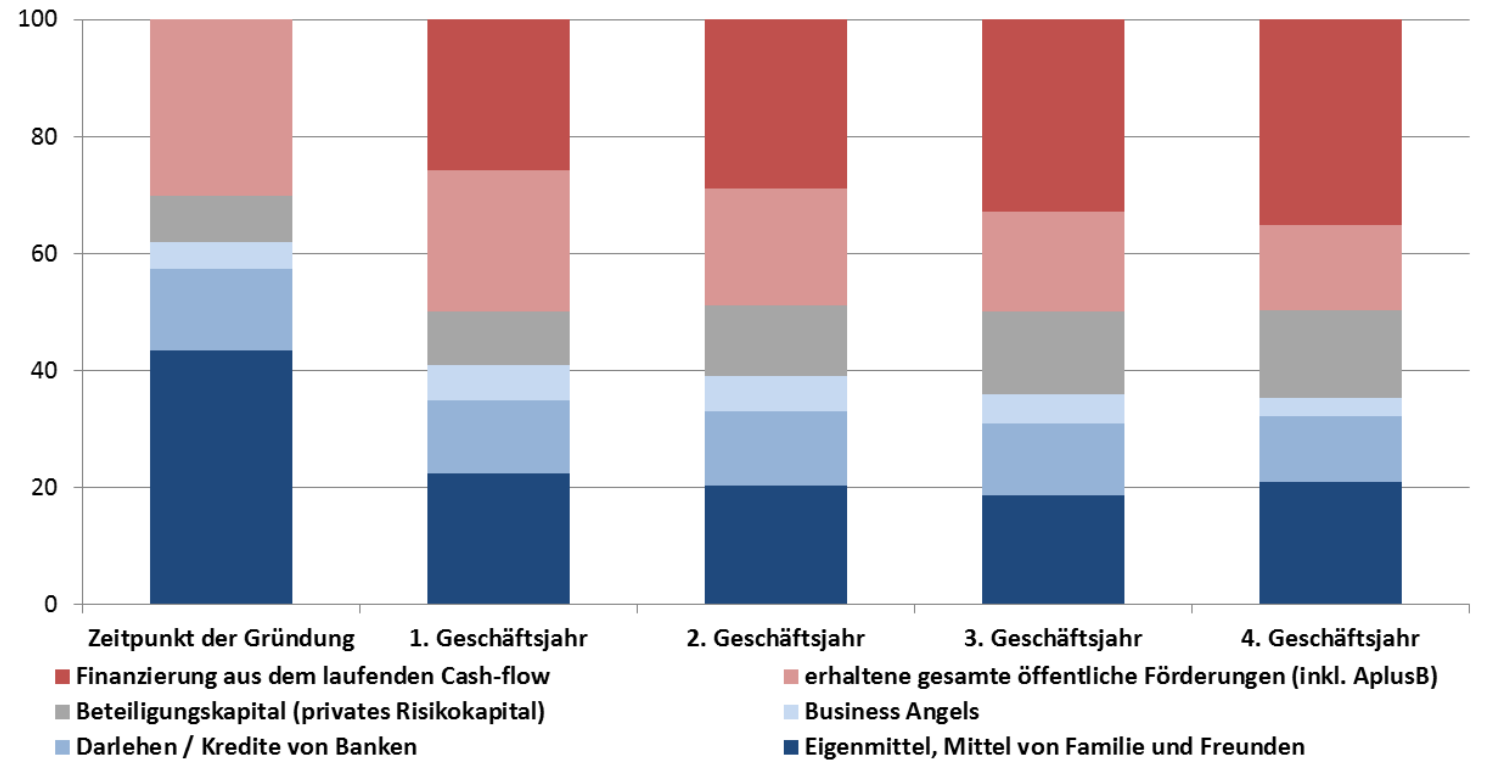

Quelle: AplusB-Gründungs-Monitoring, JR-Berechnungen 
Erste Einnahmen aus der laufenden Geschäftstätigkeit (siehe Cash Flow) erlauben, den Eigenmitteleinsatz sukzessive zurückzunehmen. Anhand der Darstellung wird aber auch deutlich, dass AplusBGründungen (im Durchschnitt) auch noch im vierten Geschäftsjahr gezwungen sind, Eigenmittel zu einem erheblichen Anteil einzubringen. Bemerkenswerterweise hat sich der angegebene ,Finanzierungsmix ‘ der AplusB-Gründungen im Programmverlauf, ungeachtet der Wirtschafts- und Finanzierungskrise, nicht wesentlich geändert. Ein (ob jährlicher Schwankungen) bedingt nachvollziehbarer Rückgang des Anteils von Beteiligungskapital und Darlehensfinanzierung wurde tendenziell durch eingeworbene Förderungsmittel kompensiert.

Im Kontext des KFW-Monitors wurde in Deutschland eine ähnliche Abfrage durchgeführt, deren Vergleich sich lohnt. Wie die Auswertungen des KfW-Gründungsmonitors zeigen, stellen für deutsche GründerInnen bürokratische Hürden und Verzögerungen (34 \% mit, $25 \%$ ohne Marktneuheit) sowie eine schwierige Auftragsakquise bzw. fehlende Kundenkontakte (25\% mit, $23 \%$ ohne Marktneuheit) die stärksten Herausforderungen im Gründungsprozess dar. Finanzierungsschwierigkeiten werden zwar von verhinderten GründerInnen als primäre Schwierigkeit angegeben, für tatsächliche GründerInnen erscheinen diese jedoch etwas weniger relevant. Der Mangel an qualifiziertem Personal bzw. MitarbeiterInnen erscheint weniger stark ausgeprägt als bei AplusB-GründerInnen.

\section{Abbildung 23 Schwierigkeiten im Gründungsprozess (Anteil in \%) in Deutschland}

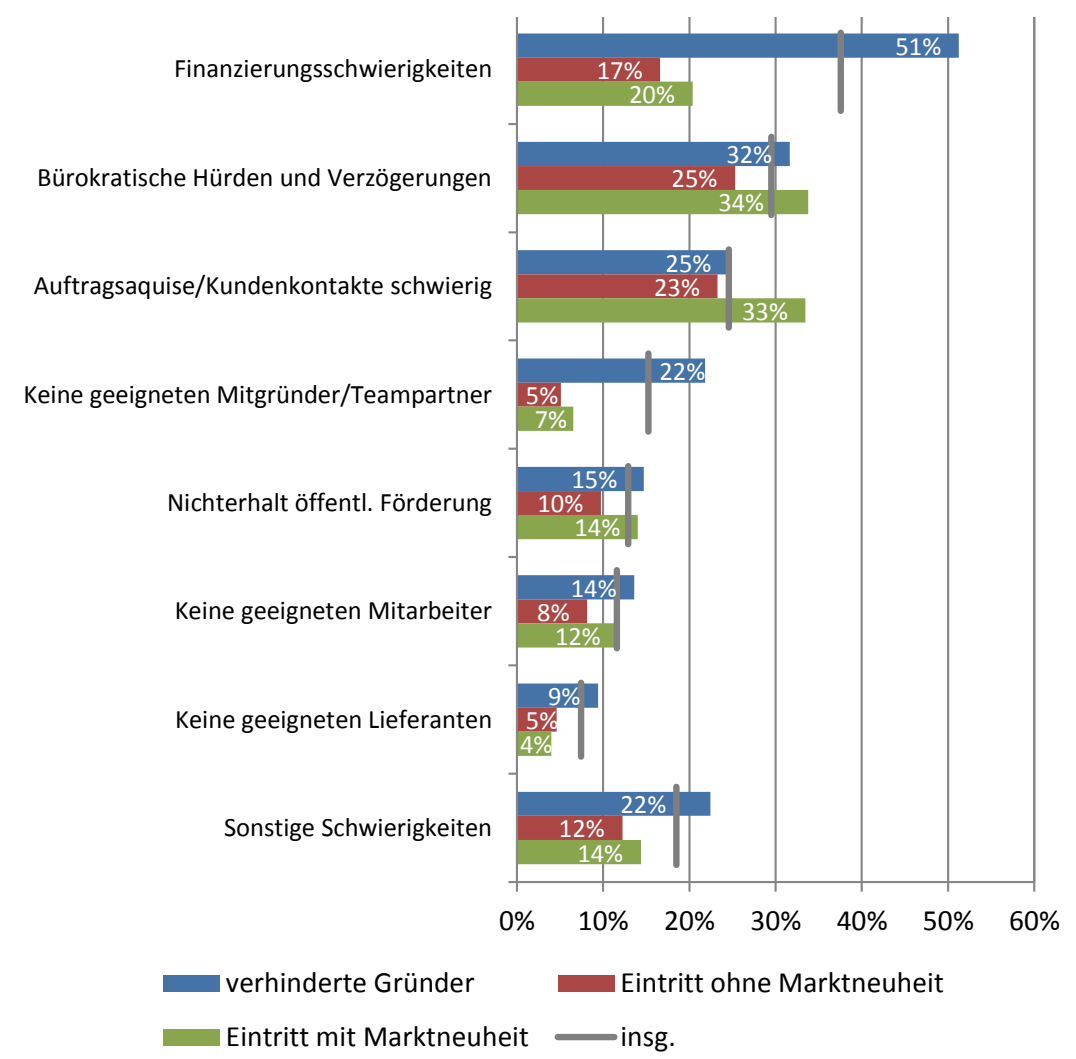

Mehrfachnennungen möglich, hochgerechnete Werte.

Quelle: KfW-Gründungsmonitor 2008-2010, Berechnungen des ZEW

Der Vergleich mit dem KfW-Gründungsmonitor zeigt zudem, dass das - auch in der öffentlichen Diskussion oftmals vorgebrachte - potentielle Risiko eines Scheiterns für GründerInnen ähnlich wie bei AplusB-GründerInnen weniger eine Rolle zu spielen scheint. Der Einschätzung der konjunkturellen Lage sowie Bedenken bezüglich einer Belastung für die Familie wird im Zuge des Gründungsprozesses erhöhte Aufmerksamkeit geschenkt. 
Abbildung 24 Bedenken im Gründungsprozess (Anteil in \%)

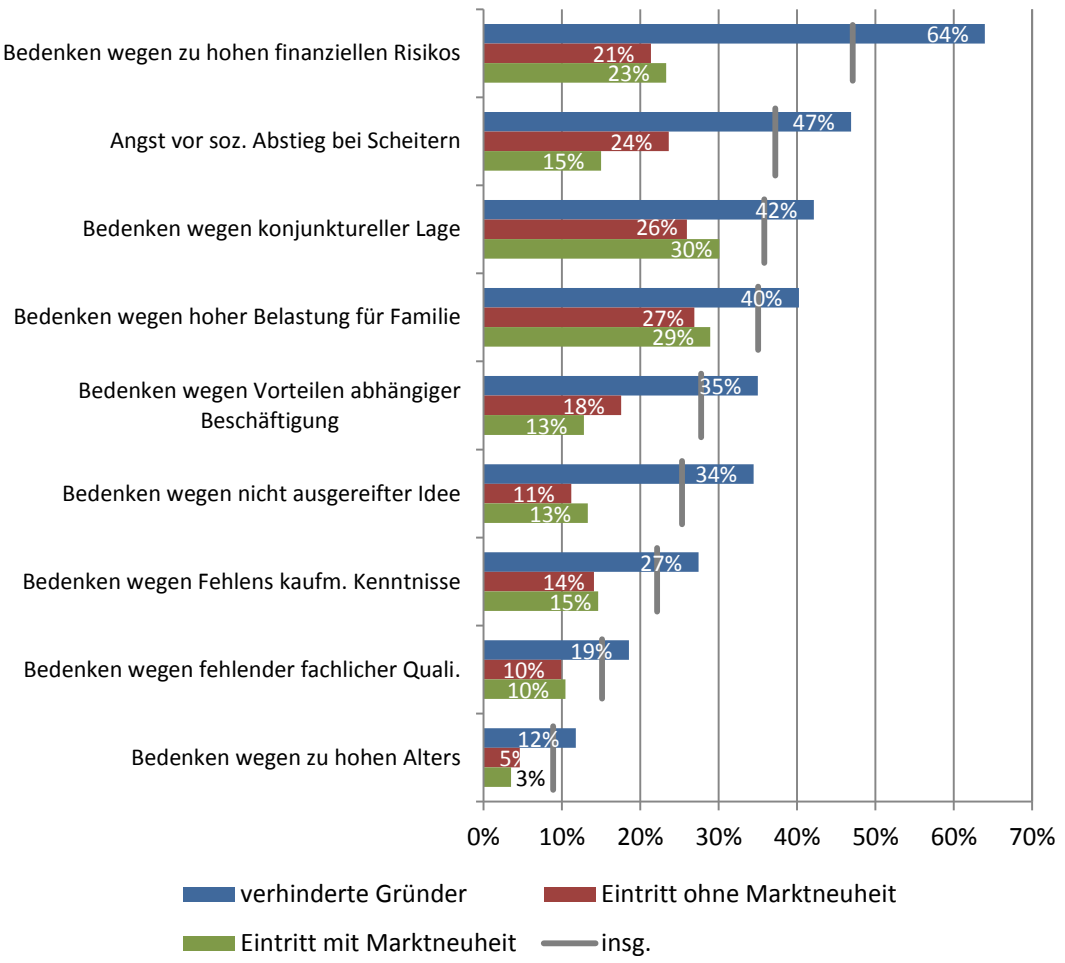

Quelle: KfW-Gründungsmonitor, Berechnungen des ZEW

\subsubsection{Verhaltensadditionalität - Wie hätten GründerInnen ohne AplusB gehandelt?}

Fragt man die AplusB-GründerInnen, wie sie ohne das AplusB-Programm gehandelt hätten, so wäre ein Drittel der Gründungen ohne AplusB gar nicht zustande gekommen. Die Hälfte der befragten Gründungen hätte versucht, die Gründung in einer anderen Form durchzuführen.

Abbildung 25 Änderungen, die die AplusB-GründerInnen aufgrund der gemachten Erfahrungen im Nachhinein vorgenommen hätten (in \%)

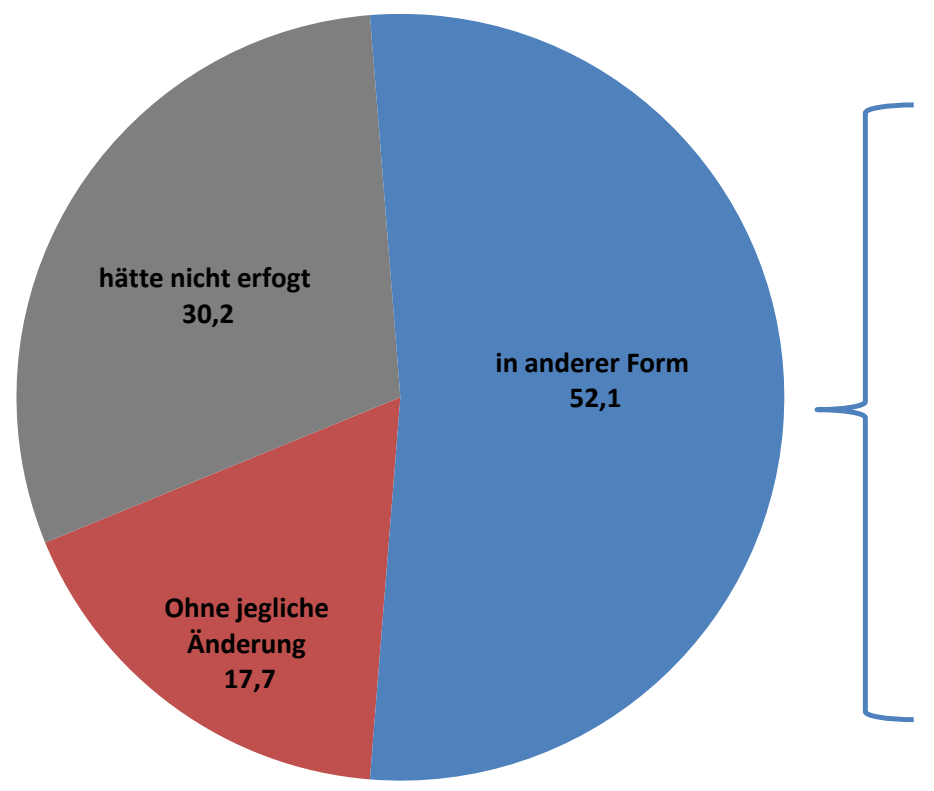

Der Gründerln hätte

andere Förderungen gewählt 30,9

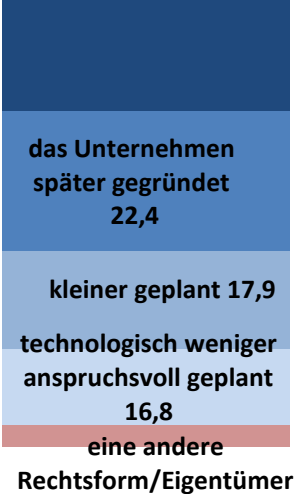

Rechtsform/Eigentüme struktur gewählt; 7,0

Quelle: AplusB-Gründungs-Monitoring, JR-Berechnungen 
Jeweils ein ähnlich großer Anteil der AplusB-Gründerinnen hätte das Unternehmen kleiner oder technologisch weniger anspruchsvoll geplant. Knapp ein Drittel der GründerInnen hätte versucht, andere Förderungen in Anspruch zu nehmen. Knapp ein Viertel der befragten AplusB-GründerInnen, die nicht in gleicher Form gegründet hätten, hätte das Gründungsvorhaben auf einen späteren Zeitpunkt verschoben. $18 \%$ der befragten GründerInnen geben schließlich an, auch ohne AplusB ohne wesentliche Änderungen gegründet zu haben.

Vergleicht man diese Angaben mit dem FFG-Wirkungsmonitoring, das laufend durchgeführt wird, so ergeben sich ähnliche Werte. Der Anteil jener, die das Vorhaben ohne die erbrachten Unterstützungsleistungen nicht durchgeführt haben, ist im Fall des AplusB-Programms etwas höher.

\subsection{Ausgründungsanlässe und Typologien von GründerInnen}

Mit den technologischen Entwicklungen, der steigenden Zahl an Spin-Offs und den sich daraus ergebenen Gründungsmöglichkeiten und -konstrukten wächst auch die Vielfalt der Ausgründungshintergründe. Wie empirische Studien ${ }^{35}$ zeigen, entstehen Gründungsprojekte klassisch vor dem Hintergrund, dass ein F\&E-Vorhaben in der akademischen Mutterorganisation nicht kommerziell verwertbar entwickelt werden kann oder dort kein Interesse dafür besteht. Die Ideen für zu entwickelnde Produkte, Verfahren oder Dienstleistungen entstammen jedoch vielfach dem privaten Umfeld der GründerInnen oder entwickeln sich außerhalb konkreter Projektzusammenhänge. Das umfasst auch das gemeinschaftliche Entwickeln von Ideen mit bereits bestehenden Unternehmen oder Personen ohne akademischen Hintergrund.

Die durchgeführten Interviews mit GründerInnen geben anekdotisch Einblick in die hohe Bandbreite an Ausgründungsanlässen. Diese reichen vom langsam gewonnen Vertrauen in die Idee, das zur Ausgründung führte, dem Erkennen von Marktlücken während einer unselbstständigen Arbeit, der Unzufriedenheit mit den Karriereaussichten bis hin zu einem allgemeinen Lebenswandel, dem Reiz, etwas Neues zu probieren und dem „Überzeugt-worden-sein“, mit anderen zusammen im Team zu gründen.

Die Entstehung und Entwicklung von Ausgründungen hängt von einer Vielzahl von Faktoren ab, die in der Literatur ${ }^{36}$ grob in drei Bereiche zusammengefasst werden: Umfeldbedingungen inkl. jene der Mutterorganisation (z.B. Standortfaktoren, Marktimpulse, Unterstützungs- und Inkubationsmöglichkeiten), das Unternehmen selbst (z.B. Idee, Konzept, Finanzierung) und die gründende Person (z.B. Demographie, Persönlichkeit, Fähigkeiten). Der Einfluss der GründerInnen gilt in der Literatur als zentralster Faktor bei der Entwicklung eines akademischen Spin-Offs. Lt. dem AplusB-GründungsMonitoring steht die Aussicht, selbstständig und unabhängig zu arbeiten, an oberster Stelle der Motivation für eine Gründung. Diese Einschätzung wird durch eine aktuellen Erhebung ${ }^{37}$ im heimischen Startup-Umfeld bestätigt, in welcher rd. $84 \%$ der Befragten angeben, dass die „Verwirklichung der eigenen Idee“ bzw. ,selbstbestimmtes Arbeiten“ (68 \%) die größte Motivation für eine Gründung sei. Höheres Einkommen und bessere Karrierechancen werden im Vergleich dazu als etwas weniger wichtig erachtet.

\footnotetext{
35 Vgl. Hemer, J; Schleinkofer, M; Göthner M. (2007) Akademische Spin-offs: Erfolgsbedingungen für Ausgründungen aus Forschungseinrichtungen. Berlin: Ed. Sigma

36 Helm, R.; Mauroner, O. (2007): Success of research-based spin-offs: state-of-the-art and guidelines for further research, in: Review of Managerial Science (RMS), Vol. 1 (2007), Nr. 3, S. 237-270;

37 Szenemonitor Tirol, Mai 2015; Befragungssample: 146 TeilnehmerInnen.
} 
Der Schritt von der Hochschule ins eigene Unternehmen muss nicht immer sofort erfolgen. Viele GründerInnen verfügten bereits über einige Jahre Berufserfahrung, was, zumindest lt. einzelnen Interviews, sich positiv auf die Unternehmensentwicklung auswirken kann. Eine diesbezügliche Studie ${ }^{38}$ zur Situation in Deutschland verweist auf das steigende Alter von High-Tech-GründerInnen. Lag der Altersschnitt 1995 bei 37,5 Jahren, waren es 2008 durchschnittlich 40,8 Jahre. In derselben Studie ergibt sich außerdem der Befund, dass nur rd. die Hälfte der High-Tech-GründerInnen über einen Hochschulabschluss verfügen. Bei den jüngeren GründerInnen kann das dadurch erklärt werden, dass sie ihren Ausbildungsweg noch nicht beendet haben. Andererseits zeigt dies auch das Potential von „NichtakademikerInnen“, Gründungsideen auf der Basis wissenschaftlicher Forschung, etwa durch Kooperation oder Fusion, umsetzen zu können.

\subsubsection{Typologien in Gründungsvorhaben}

Insbesondere im technisch-naturwissenschaftlichen Bereich scheint die Gründungsmotivation ${ }^{39}$ vielschichtig. Eine diesbezügliche Untersuchung unter universitätsnahen Technologie-GründerInnen ${ }^{40}$ konnte sechs Spin-off-GründerInnen-Typen skizzieren: Fähigkeits-ErweitererInnen, HerausforderungsSucherInnen, Expertise-NutzerInnen, Existenzsicherer, SystemherausbrecherInnen und Unabhängigkeits-StreberInnen. Diese Gruppierung lässt erkennen, dass die Gründungsmotivation zum einen in der Motivation von WissenschaftlerInnen und ForscherInnen (etwa Neugierde, Anerkennung) verortet werden, zum anderen aber auch Ausdruck einer negativen Motivation sein kann, etwa wenn die Hochschule den ForscherInnen keine entsprechenden Entwicklungsperspektiven bietet.

Im Rahmen der vorliegenden Untersuchung wurde auf Basis des AplusB-Gründungs-Monitorings mittels einer Clusteranalyse versucht, ob, und wenn ja inwiefern, eine endogene Typologie der durch $A p$ lusB-Zentren unterstützten Gründungsideen bzw. Gründungen zu identifizieren ist. Hierfür wurde ein agglomeratives, hierarchisches Clusteranalyse-Verfahren eingesetzt. Zur Clusterbildung wurden kategoriale Variablen einbezogen, die sich auf die

- Motive für die Auseinandersetzung mit einem Gründungsprojekt,

- auf die Studienrichtung der AplusB-Geförderten,

- auf die Tatsache, dass die Geförderten ein Doktorat absolviert haben und

- das Vorhandensein eines HTL-Abschlusses beziehen.

Differenziert wurde dabei nicht nach geförderten Personen, sondern nach geförderten Gründungsideen bzw. tatsächlichen Gründungen. Sollten in einem Gründungsprojekt mehrere Personen unterstützt worden sein, dann ging dieses Projekt nur einmal - mit dem besser gefüllten Datensatz - in die Analyse ein.

Aus der Analyse der oben angeführten Variablen lassen sich aus dem Pool der AplusBGründungsprojekte vier (bzw. drei und eine Restgröße) Typen von Gründungen, bzw. Gründungsideen identifizieren:

38 Vgl. Metzger, G.; Heger, D.; Höwer, D.; Licht, G (2010): High-Tech-Gründungen in Deutschland - Zum Mythos des jungen HighTech-Gründers, in Zusammenarbeit mit Microsoft, Mannheim.

39 Als relevante Gründungsmotive wurde identifiziert: Unternehmerische Herausforderung, Neugierde, Beitrag zum Gemeinwohl, Geld und Einkommen, Verwerten der eigenen Expertise und Wahrnehmen von Marktgelegenheiten, Anerkennung und Bestätigung, unzufriedenes Arbeitsumfeld und drohende Arbeitslosigkeit, Unabhängigkeit und Anwendung der Forschung; aus: Sass, Enrico (2011)

40 Sass, Enrico (2011): Der Naturwissenschaftler als Unternehmer: Gründertypen und deren Motivation im universitären Umfeld, 1. Aufl. Wiesbaden: Gabler Verlag. 


\section{Marktgetrieben (32\% der von AplusB betreuten Gründungsprojekte)}

Die Gründungsprojekte werden ganz wesentlich wegen konkreter Nachfrage begonnen. Weiterhin sehr wichtig sind in dieser Gruppe die Gründungsmotive Karriereverbesserung oder auch Einkommensverbesserung. Die Gründerpersonen sind vornehmlich in nicht-MINT-Fachrichtungen akademisch ausgebildet, WirtschaftswissenschaftlerInnen oder auch JuristInnen machen einen großen Anteil aus.

Überdurchschnittlich viele Gründungsprojekte (Vorhaben) werden erfolgreich bis zur tatsächlichen Gründung entwickelt (90\%). Diese Unternehmen weisen eine leicht überdurchschnittliche Anfangsbeschäftigung auf.

\section{Technikgetrieben (21\%)}

Bei dieser Gruppe von Gründerpersonen schlagen einzelne der abgefragten Motive nicht dominierend durch. Sie werden eher von den bestehenden Möglichkeiten (Geschäftsidee) getrieben. Hier dominieren die ingenieurwissenschaftlich ausgebildeten Personen, überdurchschnittlich häufig mit einem Doktor-Titel und meist auch bereits mit einem HTL-Abschluss.

Die Gründungsprojekte dieser Gruppe münden durchschnittlich häufig in einer Gründung (75\%) und beginnen mit einer durchschnittlichen Anfangsbeschäftigung.

\section{Forschungsgetrieben (28\%)}

Für die Gründerpersonen dieser Gruppe ist das Motiv, Forschungsergebnisse umzusetzen, von herausragender Bedeutung, aber auch die Verbesserung ihrer Einkommensaussichten motiviert sie, ein Gründungsprojekt zu beginnen. Hier finden sich hauptsächlich AkademikerInnen der Fachrichtungen Mathematik, Naturwissenschaften und Informatik sowie auch ein deutlich überdurchschnittlicher Anteil von MedizinerInnen. Ein Großteil von ihnen hat einen Doktorratsabschluss.

Mit einem Anteil von ca. $85 \%$ tatsächlicher Gründungen weist auch diese Gruppe einen überdurchschnittlichen Anteil erfolgreicher Gründungsprojekte auf. Die jungen Unternehmen dieser Gruppe betreiben überdurchschnittlich häufig selbst F\&E in den Unternehmen und haben mehr Kontakte zu Wissenschaftseinrichtungen als die Gründungen der anderen Gruppen. Die Beschäftigtenzahl bei Gründung ist unterdurchschnittlich.

\section{Nicht klar zuordenbar (19\%)}

Die verbleibende Gruppe der von AplusB betreuten Gründungsprojekte folgt keinem der abgefragten Gründungsmotive. MedizinerInnen und IngenieurInnen sind nur überaus unterdurchschnittlich in dieser Gruppe vertreten, gleichwohl haben überdurchschnittlich viele von ihnen einen HTL-Abschluss. Ebenfalls überdurchschnittlich vertreten sind Personen mit Doktorratsabschluss. Wesentlichster Befund ist, dass nur rd. ein Viertel der Gründungsprojekte dieser Gruppe mit einer tatsächlichen Gründung endet. Gründe hierfür können nur vermutet werden, etwa Rückschläge im Projekt, sinkende Motivation und Interesse.

Die ersten drei Gruppen lassen sich, ohne jedoch die Motivlage exakt vergleichen zu können, gut den eingangs erwähnten Gründertypen Fähigkeits-ErweitererInnen, Herausforderung-Suchende bzw. Expertise-NutzerInnen zuordnen. Hinsichtlich der letzten Gruppe scheint eine Überlappung mit den Typen der Existenzsicherer bzw. der System-HerausbrecherInnen möglich. Es liegt die Vermutung nahe, dass hier der Anteil jener Personen, die aus einer gewissen, vielleicht überhöhten Erwartungshaltung (siehe oben: „Reiz, etwas Neues zu probieren“) oder negativen Motivation heraus (z.B. fehlende Karriereaussichten, empfundene Alternativlosigkeit) den Weg der Selbstständigkeit einschlagen, diese dann aber nicht wie ursprünglich geplant umsetzen. 


\subsubsection{Frauen und Gründungen}

Die geringe Gründungsneigung von Frauen ist nicht nur in Österreich ein breit diskutiertes Themenfeld, welche auch von AplusB durch ein wachsendes Angeboten an gendergerechten Maßnahmen adressiert wird. Nachfolgend werden mit Fokus auf die aktuellen Studierendenzahlen an heimischen Universitäten sowie den Ergebnissen aktueller Literatur jene Aspekte skizziert, die einer höheren Gründungsaktivität von Frauen in Technologie-intensiven Bereichen entgegenstehen.

Die Anzahl der Studierenden in naturwissenschaftlich-technischen Studienfächern hat sowohl an österreichischen Universitäten wie Fachhochschulen im letzten Jahrzehnt deutlich zugenommen - sowohl bei den männlichen Studierenden, aber auch bei den weiblichen Studierenden. Wie nachfolgende Tabelle 9 zeigt, bilden Frauen dennoch weiterhin die Minderheit in den naturwissenschaftlichen Studien (37\% Frauenanteil an den Universitäten bzw. $24 \%$ an den Fachhochschulen) und insbesondere auch in den ingenieurwissenschaftlichen Studien (29\% Frauenanteil an den Universitäten bzw. $23 \%$ an den Fachhochschulen).

Tabelle 9: $\quad$ Anzahl der Studierenden an naturwissenschaftlich-technischen Studien an Universitäten und Fachhochschulen in Österreich, nach Geschlecht, für die Studienjahre 2002, 2007 und 2014

\begin{tabular}{|c|c|c|c|c|c|c|c|c|c|}
\hline & & \multicolumn{4}{|c|}{ Studierende Universitäten } & \multicolumn{4}{|c|}{ Studierende Fachhochschulen } \\
\hline & & Frauen & Männer & Summe & $\begin{array}{l}\text { Frauen- } \\
\text { Anteil in \% }\end{array}$ & Frauen & Männer & Summe & $\begin{array}{l}\text { Frauen- } \\
\text { Anteil in \% }\end{array}$ \\
\hline \multirow{3}{*}{$\begin{array}{l}\text { Ingenieur- } \\
\text { wesen, Ver- } \\
\text { arbeitendes } \\
\text { Gewerbe } \\
\text { und Bauge- } \\
\text { werbe }\end{array}$} & 2002 & 6.011 & 21.721 & 27.732 & 21,7 & 870 & 4.813 & 5.683 & 15,3 \\
\hline & 2007 & 8.748 & 24.687 & 33.435 & 26,2 & 1.633 & 6.696 & 8.329 & 19,6 \\
\hline & 2014 & 12.458 & 30.518 & 42.976 & 29,0 & 2.801 & 9.528 & 12.329 & 22,7 \\
\hline \multirow{3}{*}{$\begin{array}{l}\text { Naturwis- } \\
\text { senschaften }\end{array}$} & 2002 & 11.111 & 20.829 & 31.940 & 34,8 & 530 & 1.722 & 2.252 & 23,5 \\
\hline & 2007 & 14.744 & 25.742 & 40.486 & 36,4 & 816 & 3.027 & 3.843 & 21,2 \\
\hline & 2014 & 18.446 & 30.855 & 49.301 & 37,4 & 1.281 & 4.032 & 5.313 & 24,1 \\
\hline
\end{tabular}

Quelle: uni:data warehouse, eigene Berechnungen

In Österreich sind, 1t. F\&E-Erhebung 2013, 40.425,5 Personen als vollzeitäquivalente WissenschaftlerInnen beschäftigt - darunter sind 9.286,3 Wissenschaftlerinnen. Dies bedeutet, dass rd. $23 \%$ aller heimischen WissenschaftlerInnen Frauen sind. 2002 waren es noch $16 \%$. Die beiden größten F\&ESektoren in Österreich unterscheiden sich signifikant hinsichtlich der Partizipation von Frauen: Während der Hochschulsektor durch einen vergleichsweise hohen Wissenschaftlerinnenanteil gekennzeichnet ist (34\%), sind im Unternehmenssektor nur rd. $16 \%$ der WissenschaftlerInnen Frauen.

Allerdings zeigt sich, dass auch in diesen beiden Sektoren eine geschlechtsspezifische Segregation festgestellt werden kann: So ist in den Technologie-intensiven F\&E-Bereichen des Unternehmenssektors der Wissenschaftlerinnen-Anteil deutlich niedriger als in den wissensintensiven Dienstleistungsbereichen (siehe Tabelle 10). Im letzteren Sub-Sektor beträgt der Frauenanteil $22 \%$ und im produzierenden Bereich nur $12 \%$. Im IKT-Sektor liegt der Frauenanteil sogar nur bei $11 \%$. Die österreichischen Universitäten sind ebenfalls durch eine hohe Segregation des wissenschaftlichen Personals gekennzeichnet: Frauen stellen nur $18 \%$ der IngenieurwissenschaftlerInnen und nur $27 \%$ der NaturwissenschaftlerInnen, die an österreichischen Universitäten beschäftigt sind. 
Tabelle 10: $\quad$ Frauenanteil am wissenschaftlichen Personal in F\&E nach Sektoren für die Jahre 2002 und 2013 (in \%)

\begin{tabular}{|l|c|l|l|l|l|l|}
\hline & $\begin{array}{l}\text { F\&E-Sektor } \\
\text { insgesamt }\end{array}$ & $\begin{array}{l}\text { Hochschul- } \\
\text { sektor }\end{array}$ & $\begin{array}{l}\text { Unternehmens- } \\
\text { sektor }\end{array}$ & $\begin{array}{l}\text { Sachgüter- } \\
\text { erzeugung* }\end{array}$ & $\begin{array}{l}\text { Dienst- } \\
\text { leistungen* }\end{array}$ & $\begin{array}{l}\text { IKT } \\
\text { Sektor* }\end{array}$ \\
\hline $\mathbf{2 0 0 2}$ & 15,8 & 27,0 & 9,7 & 12,3 & 19,8 & 10,6 \\
\hline $\mathbf{2 0 1 3}$ & 23,0 & 34,8 & 15,7 & 11,8 & 22,4 & 11,4 \\
\hline
\end{tabular}

* Diese Daten umfassen das gesamte F\&E-Personal und nicht nur das enger gefasste wissenschaftliche Personal. Quelle: F\&E-Erhebungen, Statistik Austria, eigene Berechnungen

Insgesamt ergibt sich daher der Befund, dass Frauen in F\&E und insbesondere in den stärker naturwissenschaftlich-technisch geprägten Bereichen unterrepräsentiert sind. Dies zeigt, dass der geringe Anteil an GründerInnen im technologischen Feld zunächst darin begründet liegt, dass die Partizipation von Frauen in naturwissenschaftlich-technischen Ausbildungen sowie Beschäftigungen nach wie vor vergleichsweise gering ist.

Trotzdem ist der Frauenanteil bei den AplusB-GründerInnen deutlich niedriger als der Frauenanteil bei Studierenden der Natur- und Ingenieurwissenschaften sowie der Wissenschaftlerinnen in diesen beiden Bereichen an österreichischen Universitäten und der Wissenschaftlerinnen im Unternehmenssektor. Es kann daher davon ausgegangen werden, dass neben dieser geschlechtsspezifischen Ausbildungs- und Berufswahl noch weitere soziale wie ökonomische Barrieren existieren, die einer höheren Gründungsaktivität von Frauen in Technologie-intensiven Bereichen entgegenstehen. Im Folgenden sollen einige dieser Barrieren, die in der Literatur häufig Erwähnung finden, angeführt werden.

Auf Grund der vergleichsweise stark ausgeprägten horizontalen wie vertikalen Segregation im FTIBereich gelangen Frauen in ihren Karriereverläufen seltener in Positionen, die wichtige Voraussetzungen für eine Gründungstätigkeit bieten. Sie verfügen daher im Durchschnitt über weniger soziale wie ökonomische Ressourcen (Netzwerke sowie Human- und Finanzkapital), die wichtige Voraussetzungen für erfolgreiche Gründungen sind. ${ }^{41}$ Zudem sind Business Angels und Risikokapitalgeber zurückhaltender dabei, Gründungen von Frauen finanziell zu unterstützen. ${ }^{42}$ Gleichzeitig nehmen Frauen auch seltener Kredite in Anspruch und gründen Unternehmen, für die weniger Start- und Investitionskapital notwendig ist. Letzteres wird häufig mit einer sozialisatorisch anerzogenen geringeren Risikobereitschaft und einem geringeren Selbstvertrauen in Verbindung gebracht. ${ }^{43}$ Darüber hinaus führt die gerin-

${ }^{41}$ Vgl. Schiffbänker, H., Woitech, B., Gassler, H., Heckl, E., Dörflinger, A. u. Dörflinger, C. (2007), Gründerinnen in Österreich: Vergleich internationaler Förderungsmodelle für forschungs- und technologieintensive Unternehmensgründungen, Vienna; Piacentini, M. (2013), Women Entrepreneurs in the OECD: Key Evidence and Policy Challenges, OECD Social, Employment and Migration Working Papers, Vol. 147, Paris.

42 Vgl. OECD (2004), Women's entrepreneurship: Issues and policies, Paris; Cain Miller, C. (2010), Out of the loop in Silicon Valley. New York Times, 18 April 2010; Ranga, M. u. Etzkowitz, H. (2010), "Athena in the World of Techne. The Gender Dimension of Technology, Innovation and Entrepreneurship", Journal of Technology Management \& Innovation; Vol 5, No 1 (2010), Vol. 5 No. 1, pp. 1-12.; Bruin, A.d. u. Flint-Hartle, S. (2005), "Entrepreneurial women and private capital: The New Zealand perspective. International Journal of Entrepreneurial Behavior \& Research”, Int Jrnl of Ent Behav \& Res, Vol. 11 No. 2, pp. 108-128.

43 Vgl. Schiffbänker, H., Woitech, B., Gassler, H., Heckl, E., Dörflinger, A. u. Dörflinger, C. (2007), Gründerinnen in Österreich: Vergleich internationaler Förderungsmodelle für forschungs- und technologieintensive Unternehmensgründungen, Vienna; Brindley, C. (2005), "Barriers to women achieving their entrepreneurial potential: Women and risk. International Journal of Entrepreneurial Behavior \& Research", Int Jrnl of Ent Behav \& Res, Vol. 11 No. 2, pp. 144-161; Ranga, M. u. Etzkowitz, H. (2010), "Athena in the World of Techne. The Gender Dimension of Technology, Innovation and Entrepreneurship", Journal of Technology Management \& Innovation; Vol 5, No 1 (2010), Vol. 5 No. 1, pp. 1-12. 
ge Zahl an Gründerinnen im technologischen Feld zu einem Fehlen von entsprechenden weiblichen Rollenvorbildern ${ }^{44}$, die als Inspiration sowie Erfahrungsquelle dienen könnten.

Insgesamt verweist nicht nur die geringe Zahl an Frauen in naturwissenschaftlich-technischen Berufen, sondern auch die kulturell geprägte Konnotation von Technik mit Männlichkeit darauf, dass dieses Feld durch männliche Normen geprägt ist. Dies gilt insbesondere auch für den Bereich der Gründungen. Denn der Entrepreneur wird zumeist als zielstrebig, risikobereit, leistungswillig und durchsetzungsfähig vorgestellt. Diese Eigenschaften sind aber nicht geschlechtsneutral, sondern vor allem mit kulturellen Bildern und Vorstellungen von Männlichkeit und Männern assoziiert. ${ }^{45}$ Frauen wird die Übernahme der Rolle des Entrepreneurs nicht zugetraut und gleichzeitig trauen sich Frauen oftmals selbst nicht zu, diese Rollen zu übernehmen und nehmen daher sich eröffnende Möglichkeiten seltener wahr. Es müssen daher auch die kulturell geprägten Vorstellungen und Stereotype verändert werden, wenn Frauen dazu motiviert werden sollen, aus ihren Ideen Gründungen und Unternehmen entstehen zu lassen.

44 Vgl. Piacentini, M. (2013), Women Entrepreneurs in the OECD: Key Evidence and Policy Challenges, OECD Social, Employment and Migration Working Papers, Vol. 147, Paris.

45 Vgl. Brush, C.G., Bruin, A.d. u. Welter, F. (2009), “A gender-aware framework for women's entrepreneurship”, International Journal of Gender and Entrepreneurship, Vol. 1 No. 1, pp. 8-24; Schiffbänker, H., Woitech, B., Gassler, H., Heckl, E., Dörflinger, A. u. Dörflinger, C. (2007), Gründerinnen in Österreich: Vergleich internationaler Förderungsmodelle für forschungs- und technologieintensive Unternehmensgründungen, Vienna; Bruni, A., Gherardi, S. u. Poggio, B. (2004), "Entrepreneur-mentality, gender and the study of women entrepreneurs", Journal of Organizational Change Management, Vol. 17 No. 3, pp. 256-268. 


\section{Wirkung und Wirkungsweise des Programms AplusB und der geförderten Zentren}

Im Folgenden werden Wirkung und Wirkungsweise anhand von qualitativen Fallstudien sowie von sekundärstatistischen Analysen durchgeführt, die auf der Identifikation von AplusB-Einzelfällen auf unterschiedlichen Datenquellen der Statistik Austria sowie von FFG und aws aufbauen. Diese Betrachtungen sollen das systemische Zusammenspiel von mitgebrachten Voraussetzungen sowie spezifischen Herausforderungen der einzelnen Vorhaben sowie der relevanten Umfeld-Ereignisse und der Unterstützungsleistungen im Rahmen von AplusB illustrieren. Des Weiteren werden robuste Zahlen ermittelt, die klarstellen sollen, inwieweit sich AplusB-Unternehmen nach der erfolgten Gründung tatsächlich erfolgreicher als Gründungen, die nicht im Rahmen von AplusB unterstützt wurden, entwickeln. Davon leiten sich Wirkungen auf Wachstum, Beschäftigung, Innovation und Wettbewerbsfähigkeit ab.

\subsection{Herausforderungen und Mehrwert des Programms am Beispiel ausgewählter Fallstudien}

Im Rahmen der Evaluierung wurden sieben Fallstudien zu Unternehmensgründungen durchgeführt, sowohl zu Unternehmen, die Unterstützung von AplusB-Zentren erhalten haben, als auch Unternehmen, die ohne Unterstützung des AplusB-Programms gegründet wurden.

Als Basis wurden folgende Kriterien erarbeitet, mit dem Ziel, einen guten Mix von unterschiedlichen Unternehmensgründungen zu erhalten: Region, Branche, Gründungskonstellation und Geschlecht der GründerInnen sowie das Gründungsjahr. Für die Festlegung der Unternehmen wurden die Gründungen der AplusB-Zentren berücksichtigt sowie die Übersichtskarten der Austrian Startups und Startupblink $^{46}$, PreisträgerInnen des 12b-Wettbewerbs, Recherchen und Gespräche mit VertreterInnen von Technopolen, Clustern, Kompetenzzentren sowie den Technologietransfer-Zentren der Universitäten. Folgende Unternehmen wurden in Abstimmungen mit den Auftraggebenden ausgewählt:

Tabelle 11 Die Fallstudienbeispiele im Überblick

\begin{tabular}{|l|l|l|l|l|l|l|}
\hline Unternehmen & $\begin{array}{c}\text { AplusB- } \\
\text { Zent- } \\
\text { rum }\end{array}$ & Region & $\begin{array}{c}\text { Grün- } \\
\text { dungs- } \\
\text { jahr }\end{array}$ & $\begin{array}{c}\text { Grün- } \\
\text { derIn }\end{array}$ & $\begin{array}{c}\text { Team- } \\
\text { grün- } \\
\text { dung }\end{array}$ & \multicolumn{1}{|c|}{ Branche } \\
\hline sticklett & build! & Ktn. & 2014 & $\mathrm{w}$ & & Produkt: Kleidung/IT \\
\hline MTA-Systems & Tech2b & OÖ & 2010 & $\mathrm{~m}$ & Ja & Produkt: Antriebstechnologie \\
\hline AFreeze GmbH & CAST & Tirol & 2008 & $\mathrm{~m}$ & Ja & Produkt: Medizintechnik \\
\hline Marinomed & INITS & W & 2006 & w/m & Ja & Produkt: Biotechnologie \\
\hline $\begin{array}{l}\text { PRSG : Pharmaceutical and } \\
\text { Regulatory Services GmbH }\end{array}$ & - & Stmk. & 2012 & $\mathrm{w}$ & Ja & $\begin{array}{l}\text { Dienstleistung: Consulting im } \\
\text { Pharma-Bereich }\end{array}$ \\
\hline NaKu & - & NÖ & 2010 & $\mathrm{~m} / \mathrm{w}$ & Ja & $\begin{array}{l}\text { Produkt: Natürliche Kunst- } \\
\text { stoffe }\end{array}$ \\
\hline LOGMEDIA & - & Ktn. & 2006 & $\mathrm{~m}$ & & Dienstleistung: IT / Software \\
\hline
\end{tabular}

Quelle: Darstellung convelop

46 www.austrianstartups.com/ecosystem/ sowie www.startupblink.com/ 
Ein zentrales Differenzierungsmerkmal zwischen den ausgewählten Unternehmen betrifft den persönlichen Hintergrund und bestehende Erfahrungen vor der Gründung. Während zwei Unternehmen bereits auf Gründungserfahrungen aufbauen konnten $\left(2^{47}\right)$, sind andere direkt aus der wissenschaftlichen Tätigkeit an der Universität hervorgegangen (3) oder wurden aus der Berufserfahrung in Unternehmen entwickelt (3).

Darüber hinaus befinden sich die Unternehmen in unterschiedlichen Phasen ihrer Entwicklung. Während ein Unternehmen noch ganz am Beginn steht und ein Unternehmen derzeit in der Phase der Produktüberleitung ist, konnten andere schon am Markt Fuß fassen. Zwei Unternehmen mussten bereits Umstrukturierungen aufgrund von Markteinbrüchen vornehmen (2). In einem Fall wurden aufgrund des MitarbeiterInnen-Wachstums mittlerweile organisationale Veränderungen (Einführung einer mittleren Management-Ebene) durchgeführt.

\subsubsection{Wer gründet? Wie kommt es zur Gründung?}

Gründungsideen entstehen, indem Bedarfe für innovative Lösungen durch persönliche Erfahrungen identifiziert (1) oder durch Forschungsergebnisse, die für Anwendungen weiterentwickelt werden (1). Individuelle inhaltliche/technische Kompetenzen dienen bei der Entwicklung von Produkten und Leistungen als Anknüpfungspunkte, wobei oft gerade über interdisziplinäre Zugänge innovative Ideen entstehen (1).

Die Motivation, selbstständig zu sein, kann bereits in der Ausbildung klar feststehen (1) oder mitgedacht werden (1), sie kann sich durch berufliche Vorerfahrungen entwickeln (3) oder durch externe Vorgaben gezielt gefördert werden (1 - geplante Ausgründung aus K-Zentrum). Das persönliche Umfeld beeinflusst die Gründungsentscheidung wesentlich. Gründungserfahrung anderer Familienmitglieder (2), bewusste Abgrenzung von üblichen Berufswegen (1), ein Team von Gleichgesinnten (2) sowie externe Fördernde (2) sind unterstützende Faktoren, um den Schritt in die Selbstständigkeit zu wagen.

\subsubsection{Wo liegen die gemeinsamen Herausforderungen in der Gründung und in den Entwicklungsphasen?}

Eine Unternehmensgründung ist ein intensiver Prozess, der die GründerInnen fordert, Ideen in vermarktbare Leistungen und Produkte umzuwandeln, ein Businessmodell zu entwickeln, die notwendigen Kompetenzen und Ressourcen aufzubauen, Zielgruppen und Markt zu identifizieren und entsprechend anzusprechen. Dies ist kein linearer Prozess, denn die Dynamik der Rahmenbedingungen und Entwicklungsprozesse veranlassen eine laufende Weiterentwicklung und Adaptierung des Geschäftsmodells. Im Spannungsfeld von Fokussierung auf das Kerngeschäft und dem Adaptierungsbedarf wird dieser Prozess anfangs vielfach zu optimistisch eingeschätzt.

Die GründerInnen benötigen daher Zeit, starke Nerven, Reflexionsfähigkeit, hohe Lernbereitschaft und vor allem ein gutes Netzwerk mit kompetenten, engagierten ExpertInnen für die unterschiedlichen Fragen und Anforderungen. Die Möglichkeit, sich mit anderen GründerInnen auszutauschen, wurde als sehr wertvoll betont (2).

UnternehmerInnen, die mit Unterstützung eines AplusB-Zentrums gegründet haben, betonen das hohe Engagement der ZentrumsmitarbeiterInnen bei der Vernetzung mit den richtigen ExpertInnen und PartnerInnen und bei Fragen zum Businessmodell, insbesondere zu den Themen IPR und Finanzierung.

\footnotetext{
${ }^{47}$ Die Zahlen in Klammern (n) geben die Zahl der Fallstudien an, welche den jeweiligen Zusammenhang explizit erwähnt haben.
} 
Für zwei der befragten UnternehmerInnen, die ohne AplusB-Zentrum gegründet haben, spielen private Netzwerke eine große Rolle (2).

Die Thematik der Finanzierung hängt eng mit der Frage der Vernetzung zusammen, wobei es den befragten GründerInnen wichtig ist, die Interessen und Branchenkenntnisse von FinanzierungspartnerInnen zu berücksichtigen. So wurde in einem Fall die Suche nach geeigneten InvestorInnen, die sich über fast ein Jahr erstreckte, aufgrund mangelnder Anerkennung der erbrachten Vorleistungen und Ansprüche im IPR-Bereich wieder abgebrochen. Der benötigte Finanzierungsbedarf wurde durch den privaten Freundeskreis mitunterstützt (1). Generell wird festgestellt, dass Finanzierungsfragen in den unterschiedlichen Entwicklungsphasen wiederholt auftreten.

Wachstum ist ein ursächliches Ziel der Gründung aller Unternehmen. Für die weitere Entwicklung ist deshalb die Frage passender Humanressourcen und deren Finanzierung eine wesentliche Herausforderung.

Typische Herausforderungen sind:

- Marktzugang: Der Weg zu den KundInnen ist eine entscheidende Herausforderung für alle befragten Unternehmen. Von Vorteil ist, wenn aus bisherigen Erfahrungen bzw. dem vorangegangenen Werdegang bereits potenzielle KundInnen bekannt sind (2). Aber selbst in diesen Fällen verhalten sich potenzielle KundInnen gegenüber neu gegründeten Unternehmen zunächst zurückhaltend, bis die GründerInnen bewiesen haben, dass sie sich stabil am Markt positionieren können. Insbesondere in der Startphase bedarf es daher eines langen Atems, um einen Markt aufzubauen. In spezifischen Branchen (bspw. Pharma, Medizintechnik) sind für die Markterschließung Qualitätsstandards nachzuweisen, beispielsweise durch Zertifizierungen (wie CE-Kennzeichnung) oder Studienergebnisse (toxikologische/klinische Studien).

- Umsatzerwartung in Startphase: Der Optimismus des Gründungsprozesses bringt vielfach allzu optimistische Einschätzungen in den Entwicklungsplänen mit sich (4). Die Gründe für den geringeren Erfolg sind unterschiedlich: Sie reichen von signifikanten Verzögerungen bei Produktentwicklungen (3) über Probleme mit strategischen Kooperationspartnern (1) bis hin zu den bereits erwähnten Schwierigkeiten, am Markt Fuß fassen zu können. In einigen Fällen konnten diese Umsatzausfälle in der Startphase durch begleitende Aktivitäten, bspw. den Vertrieb von ergänzenden Produkten (3) bzw. Beratungsleistungen (1), ausgeglichen werden.

- Finanzielle Engpässe: Die Frage der Finanzierung ist für die Gründungen, insbesondere für Unternehmen mit längerer Entwicklungsphase, eine große Herausforderung, die während des Gründungsverlaufs wiederkehrend auftritt. Die von $\operatorname{den} A p l u s B$-Zentren teilweise zur Verfügung gestellte finanzielle Unterstützung ist hilfreich, aber nicht ausreichend. Die Identifizierung von InvestorInnen setzt entsprechende Netzwerke voraus, gleichzeitig sind bei der Einbindung von Kapitalgebenden die jeweiligen Interessen zu prüfen und abzustimmen, damit Win-Win-Situationen möglich werden (2). Die Akquise von Förderungen als weitere Finanzierungsquelle erfordert Informationen über bestehende Angebote sowie Kenntnisse in der Antragstellung. Die AplusB-Unternehmen haben dazu Unterstützung von den Zentren erhalten, während ein Unternehmen, das ohne AplusB-Unterstützung gegründet hat, bisher noch keine Förderanträge an nationale F\&E-Förderungsstellen eingereicht hat, da der Aufwand dafür als sehr hoch empfunden wird.

- Kompetenzerweiterung: GründerInnen unterscheiden sich in Bezug auf ihre Vorerfahrungen und bringen unterschiedliche Kompetenzen mit. Demensprechend divers ist der Bedarf an noch fehlenden Kenntnissen. Oftmals, jedoch nicht in jedem Fall, müssen unternehmerische Kompetenzen aufgebaut werden, um die technischen und wissenschaftlichen Kenntnisse zu ergänzen (4). Auch die Erweiterung des Teams durch Personen, die entsprechende Kompeten- 
zen mitbringen, ist eine gute Lösung (2), wobei die Persönlichkeit im Zusammenspiel mit dem gesamten Team eine wichtige Rolle spielt.

- Erkennen von Lücken und Einbindung von externer Expertise: Welches Know-how und welche Ressourcen fehlen, wird teilweise erst im operativen Geschäft sichtbar (3). Begleitende MentorInnen, die mit den Entwicklungsprozessen in den jeweiligen Bereichen vertraut sind, können frühzeitig auf diese Lücken aufmerksam machen. Die Vermittlung von ExpertInnen ist in weiterer Folge wichtig (2). Kooperationen sind ein wesentlicher Teil eines erfolgreichen Geschäftsmodells für alle Unternehmen. Herausfordernd ist zunächst, geeignete Partner zu identifizieren - hier wird das persönliche Engagement der GründerInnen von den vermittelnden Stellen (AplusB-Zentren, Wirtschaftskammer) unterstützt, was als sehr wertvoll erlebt wird.

- Zeit und Nerven: Gründen ist ein zeit- und nervenaufreibender Prozess. Unbedingt nötig sind daher neben der fachlichen Kompetenz auch laufende Motivation und ein unterstützendes familiäres/soziales Umfeld. Durch den Austausch mit anderen GründerInnen, die in ähnlichen Situationen sind, wird viel wertvolles Wissen in die Entwicklungsschritte eingebracht und die Vernetzung als motivierend erlebt.

- Schutzrechte und Zertifizierungen: IPR sind für einige Branchen (Medizintechnik, Pharma) unerlässlich und müssen sehr frühzeitig gesichert sein. Auch Zertifizierungen sind zum Teil für einige Produkte verpflichtend. Gleichzeitig können freiwillige Zertifizierungen hilfreich sein, um sich von MitbewerberInnen abzuheben (1) und dienen bei InvestorInnen und Fördergebenden als Qualitätsbeweis für die Aktivitäten und Entwicklungen (1).

\subsubsection{Einschätzungen zu den AplusB-Zentren}

\section{Zugang zu AplusB-Zentren}

Die befragten GründerInnen wurden auf unterschiedliche Weise auf die AplusB-Zentren aufmerksam. So wurde ein Unternehmen durch ein Plakat auf den Innovationspreis aufmerksam und erkannte die möglichen Unterstützungsleistungen durch AplusB aber erst, nachdem es den Innovationspreis gewonnen hatte. Ähnlich war die Situation bei einer weiteren Gründung, die erst im Anschluss an den Wettbewerb über die weiteren Unterstützungsleistungen des Zentrums in Tirol erfuhr. Ein Unternehmen wurde durch die aws auf das Zentrum in Wien aufmerksam gemacht und nahm daraufhin an einem mehrstufigen Businessplan-Wettbewerb teil. Eine Gründerin nahm ebenfalls an einem Ideenwettbewerb eines AplusB-Zentrums teil, kannte dieses jedoch bereits im Vorfeld. In einem Fall war das Gründungsprojekt über das Kompetenzzentrum sehr nahe an der Wissenschaft angesiedelt und man kannte zwar das bestehende Zentrum, es wurde jedoch in keiner Phase ein näherer Kontakt angestrebt.

\section{Reflexion der AplusB Leistungen durch die befragten GründerInnen}

Insgesamt geben jene GründerInnen, die von $A p l u s B$ unterstützt wurden, ein überwiegend positives Feedback.

- Die Beratung im Zuge der Businessplan-Erstellung wurde sehr positiv bewertet. Ohne Unterstützung wären die Businesspläne nicht in diesem Detail ausgearbeitet worden (3). Durch die laufende Unterstützung, auch in den Wettbewerben, setzten sich die GründerInnen intensiv und kontinuierlich mit der Erstellung des Businessplans auseinander. Vielfach wurde durch die Beratung erst Bewusstsein über eigene „blinde Flecken“ und fehlende Kompetenzen ermöglicht (2). Die Bedeutung der Beratung hinsichtlich einer Businessplan-Erstellung wird auch von jener Gründung posi- 
tiv wahrgenommen, deren Idee vom Zentrum abgelehnt wurde (1). Beim Detaillierungsgrad und Aufwand bei der Businessplan-Erstellung sollte jedoch bedacht werden, dass im Laufe der Gründung immer wieder Adaptierungsbedarf entsteht. Dieser kann durch geringe privatwirtschaftliche Vorerfahrung der GründerInnen gegeben sein (2), durch sich dynamisch ändernde Rahmenbedingungen entstehen oder durch die Notwendigkeit, ein bestimmtes Businessmodell (angestrebte KundInnengruppen und Vertriebskanäle) zu adaptieren (1).

- Die Schulungsangebote im Bereich IPR und Markenschutz wurden von zwei Unternehmen hervorgehoben, die im Vorfeld keinerlei Erfahrung zu diesen Themen hatten (2). Für ein weiteres Unternehmen sind Schutzrechte ebenfalls essentiell, das Gründungsteam konnte jedoch auf ExpertInnen im eigenen Netzwerk zurückgreifen.

- Betont wird das hohe Engagement des Zentrumsmanagements und der Beratenden sowie die sehr hilfreiche und gezielte Netzwerk- und Vermittlungsarbeit der AplusB-Zentren, die den Zugang zu externer und insbesondere kompetenter Expertise ermöglicht. Hierdurch konnte die Finanzplanung unterstützt (1), geeignete Immobilien gefunden (1) und eine passende Produktionspartnerschaft aufgebaut werden (1).

- Von den interviewten UnternehmerInnen haben jene, die AplusB-Unterstützung erfahren haben, auch weitere Förderkanäle genutzt, wobei sie bei der Antragstellung vom AplusB-Zentrum unterstützt wurden (2: FFG; 2: aws, FFG).

- Die inhaltlichen Unterstützungsmöglichkeiten der ZentrumsberaterInnen stoßen an ihre Grenzen, wenn sehr spezialisierte, technisch-inhaltliche Expertise nötig ist (bspw. Antriebstechnik, Zertifizierung, etc.). Gleichzeitig wird betont, dass diese Einschränkung durch gezieltes Vermitteln von benötigter externer Expertise ausgeglichen wird (im Unterschied zu anderen Beratungseinrichtungen) (2).

- Nur ein Unternehmen der vier Fallstudien zu AplusB-Gründungen ist auch tatsächlich in den Räumlichkeiten der Zentren untergebracht. In diesem Fall (Einzelgründung einer Frau mit Kindern) wird diese Lösung besonders positiv wahrgenommen, da durch die Möglichkeit, die Kinder auch ins Büro mitzunehmen, die Vereinbarkeit von Gründung und Familie erleichtert wird. Gleichzeitig werden die Vernetzung und der Austausch mit weiteren GründerInnen sehr geschätzt, dies hat bereits zur Anbahnung weiterer Kooperationen geführt.

- Das Mentoringprogramm, das einige Unternehmen (2) in Anspruch genommen haben, wird sehr positiv erlebt. Feedback durch fachlich sehr kompetente MentorInnen hat die Unternehmensplanung verbessert. Jenes Unternehmen, das zwar AplusB-Unterstützung bekommen hat, jedoch in keinem Mentoringprogramm war, hätte eben diese Unterstützung in seiner bisherigen Entwicklung als sehr wertvoll empfunden. Ein kontinuierlicher Ausbau der Mentoring-Netzwerke ist daher anzustreben, um auch Gründungsprojekte in spezifischen Marktnischen gut unterstützen zu können.

- Betriebswirtschaftliches Grundlagenwissen fehlt in vielen Fällen, jedoch bringen einige GründerInnen diese Kompetenzen mit; es sollte daher nicht grundsätzlich von fehlenden betriebswirtschaftlichen Kompetenzen ausgegangen werden. Entsprechend sollten im Bereich der Qualifizierung und Weiterbildung der GründerInnen möglichst flexible Module angeboten werden, um Know-how-Lücken schließen und bestehende Kenntnisse ausbauen zu können. 


\subsubsection{Zusammenfassende Beobachtung aus den Fallstudien}

Die Unternehmen hätten sich nach Einschätzung der GründerInnen ohne AplusB-Unterstützung weniger gut entwickelt (1), bzw. wären gar nicht gegründet worden (1). Dies untermauert noch einmal die im Rahmen des Gründungs-Monitoring beantworteten Fragen zur Additionalität des Programms. Die laufende Motivation, der Zuspruch und der Glaube der Zentren an den Erfolg der Ideen waren insbesondere in der Anfangsphase von großer Bedeutung für die Unternehmen (2).

Die Leistungen des AplusB-Programms zur Vernetzung (Vernetzungs-Veranstaltungen, Kontaktanbahnung zu potentiellen PartnerInnen und MitarbeiterInnen) sind zum Aufbau von Kooperationsbeziehungen, zum Austausch innerhalb der Gründungs-Community und zur Identifikation von notwendigen Expertisen sehr positiv zu sehen. Ohne AplusB-Unterstützung sind dazu mehr persönliches Engagement sowie ausgeprägte private Netzwerke notwendig.

Unternehmenswachstum wird als Grundsatzentscheidung (1) beschrieben. Wichtige Grundsteine dafür werden auch in den frühen Phasen der Unternehmensentwicklung gelegt (bspw. IPR: 2; Gebrauchsmusterschutz: 1). Die Aufnahme neuer MitarbeiterInnen ist vor allem auch eine finanzielle Frage. Steht ein Unternehmen noch in der Entwicklungsphase, kann Wachstum nur durch ergänzende Finanzierungen ermöglicht werden, bspw. durch Investoren (2) oder Förderungen (1). Eine Eigenfinanzierung der Unternehmenserweiterung erfordert entsprechende Umsatzleistungen (1).

\subsection{Quantitative Beurteilung der ökonomischen Wirkungen des Programms AplusB anhand eines Vergleichs von AplusB-Gründungen mit einer Kontrollgruppe.}

In einem ersten Schritt wurden die von den Zentren erfassten Gründungsvorhaben mit dem AplusBGründungs-Monitoring zusammengeführt. 140 der von den AplusB-Zentren erfassten Gründungsvorhaben konnten vom AplusB-Gründungs-Monitoring nicht erreicht werde.

Der nächste Schritt bestand in der weiteren Verknüpfung mit dem Unternehmensregister der Statistik Austria aufgrund der vorliegenden Firmenbuch und -Umsetzsteuernummern sowie einem direkten Abgleich von Unternehmensnamen, unterstützt durch Internetrecherchen. Die durchgeführten ,händischen" Abgleiche waren aufwendig, sie ermöglichten jedoch, dass $83 \%$ der laut AplusB-GründungsMonitoring identifizierten, formal realisierten $A p l u s B$-Gründungen auch in der offiziellen Statistik identifiziert werden konnten. Der damit aufgebaute Datensatz bietet eine sehr aussagekräftige Grundlage für weitere Analysen. Verknüpfung der Datenbasen wurde beispielsweise bereits für die branchendifferenzierte Betrachtung der im Rahmen des AplusB-Gründungs-Monitoring identifizierten Unternehmen genutzt.

Die FFG pflegt seit relativ kurzer Zeit eine enge Schnittstelle mit dem Unternehmensregister der Statistik Austria. Während aktuell in Begleitung zum Eintrag in die Transparenzdatenbank jeder neu hinzukommende Förderfall der FFG im Unternehmensregister identifiziert wird, ist die Erfassungsquote vor 2012 geringer. Ungeachtet dessen haben nicht alle AplusB-GründerInnen eine FFG-Förderung beantragt oder in Anspruch genommen. Von den 530 eindeutig im Unternehmensregister identifizierten AplusB-Unternehmen konnten dennoch 417 auch in der FFG-Datenbank identifiziert werden. Die Erfassungsquote in den ersten Programmjahren ist nur geringfügig niedriger, weswegen auch hier eine sehr zuverlässige Datenquelle vorliegt. 
Abbildung 26 Verknüpfung der eingesetzten Register und Sekundärdatenquellen

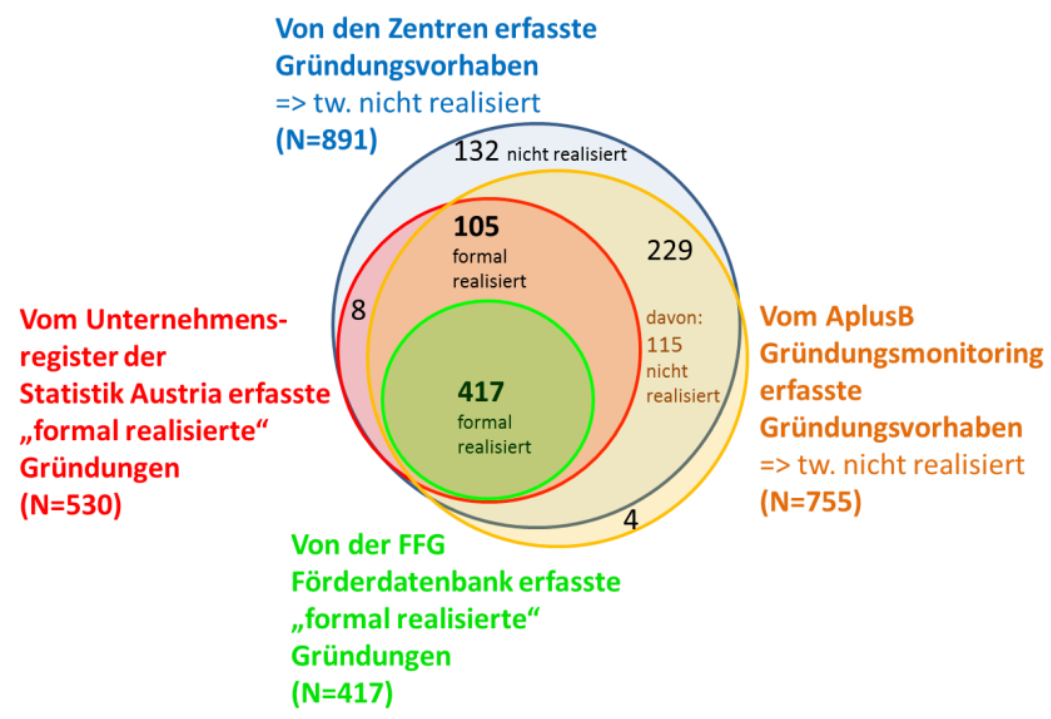

Quelle: FFG-Datenbank, AplusB-Zentren, AplusB-Gründungs-Monitoring, Unternehmensregister der Statistik Austria, Berechnungen JR

Die folgende Graphik zeigt die Zahl der jeweils bis zum Ende der Jahre 2007 bis 2013 gesamt gegründeten und zumindest ein Jahr beständigen AplusB Unternehmen. ${ }^{48}$ Bis zum Ende des Jahres 2013 wurden 486 AplusB Unternehmen gegründet, die auch den Beobachtungszeitraum der offiziellen Statistik von einem Jahr überstanden haben. Der Outcome des AplusB Programms an Gründungen belief sich auf 30 bis 53 Gründungen.

Abbildung 27 Jeweils bis zu den Bezugsjahren 2006 bis 2013 kumulierte Zahl der AplusB Gründungen

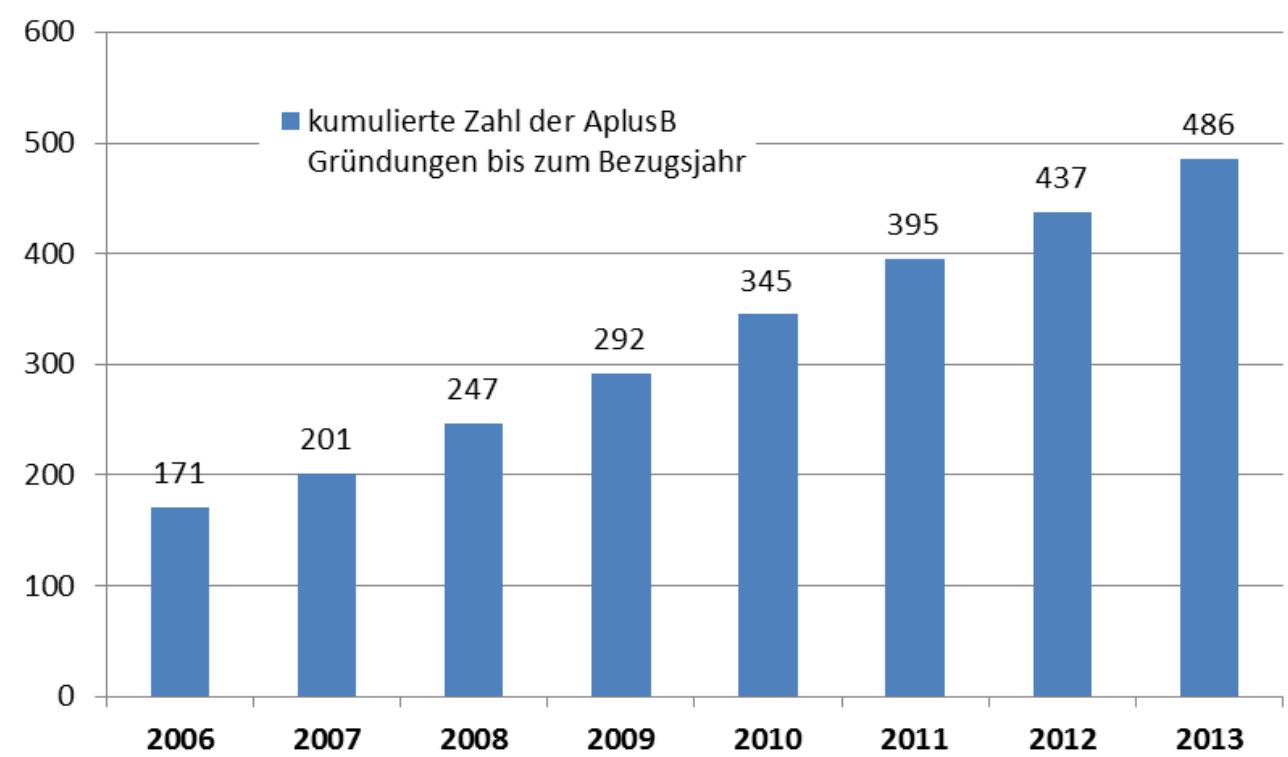

Quelle: Statistik Austria, eigene Berechnungen

\footnotetext{
${ }^{48}$ Die offizielle Statistik zählt Gründungen erst, wenn Sie nach dem Gründungsvorgang mindestens ein Jahr Bestand haben. Dies ist auch der Grund, warum die Gründungsstatistik Zahlen mit einem Jahr Verzögerungen veröffentlicht (aktuell 2013).
} 


\subsubsection{Quantitative Analyse der Wirkung auf Überleben, Wachstum, Beschäftigung und F\&E Performance}

Auch wenn die Branchenzugehörigkeit einzelner AplusB-Unternehmen auf der Ebene von NACEDreistellern erfolgt ist, musste mit Blick auf die Robustheit der Analyseergebnisse, aber auch auf die Datenschutzbestimmungen hierfür, eine Zusammenfassung von verwandten Branchengruppen erfolgen. Einzelne Branchebereiche, die im AplusB-Programm sehr stark vertreten sind (bspw. die Erbringung von Dienstleistungen der Informationstechnologie) werden aus den festgelegten Aggregaten herausgehoben und separat betrachtet. Des Weiteren ist es notwendig, mit Blick auf einen hinreichenden Zellenbesatz jeweils zwei bis drei Gründungsjahre zu einer Gründungskohorte zusammenfassen (siehe Abbildung 28).

Abbildung 28 Branchenabdeckung einzelner Gründungskohorten in der Programmlaufzeit, absolut

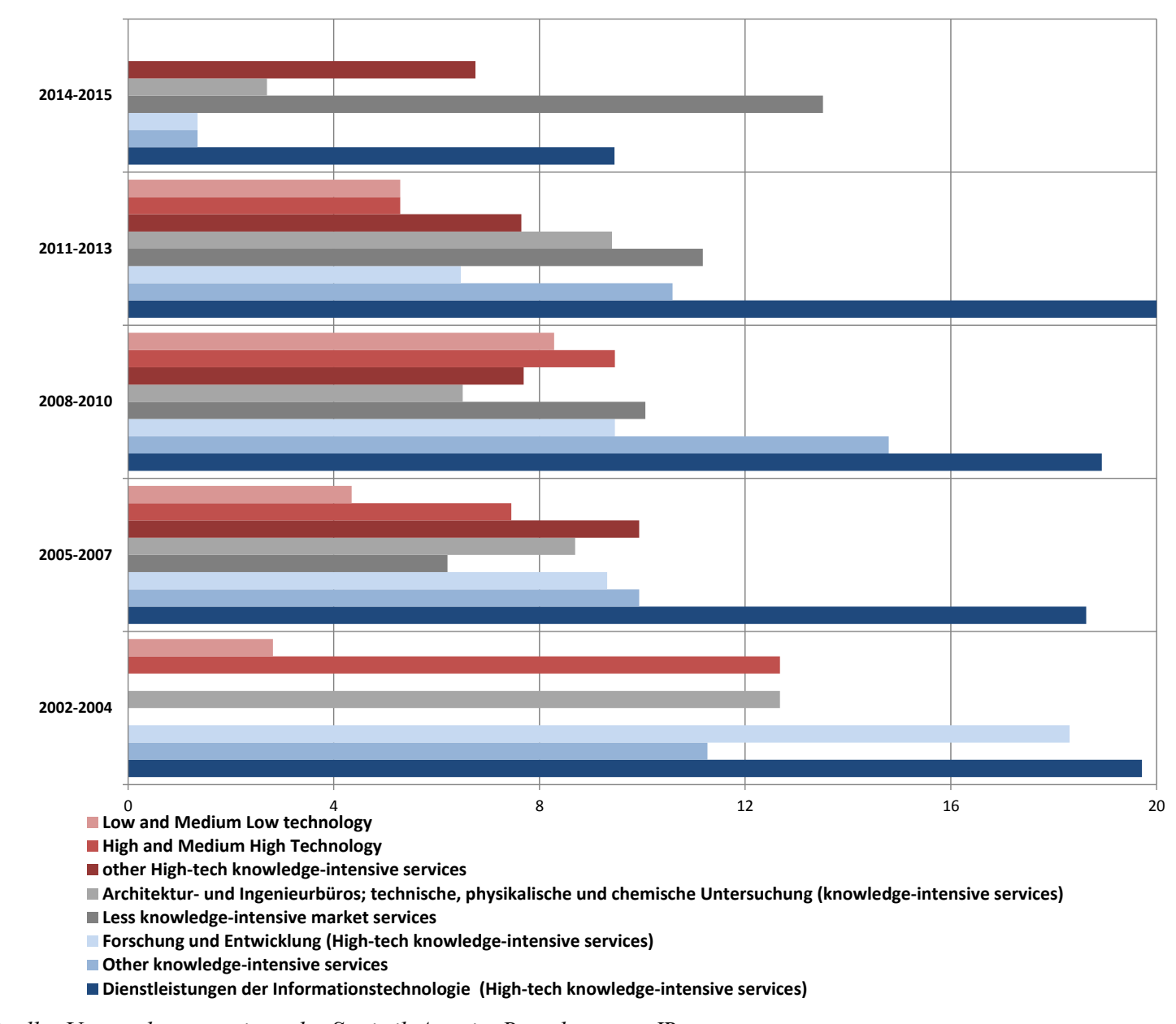

Quelle: Unternehmensregister der Statistik Austria, Berechnungen JR

\section{Die Überlebensfähigkeit von AplusB-Gründungen im Vergleich}

Die Förderung von Unternehmensgründungen setzt bei der Initiierung von Unternehmen, beim Überleben sowie beim Wachstum (in erster Linie hier bei der Wettbewerbsfähigkeit) an. Mit der Inkubation und einer intensiven Vor-Ort-Betreuung, die zu einem wesentlichen Teil in der Phase vor der gewerberechtlichen Gründung ansetzt, stellt sich die Frage, inwieweit AplusB-Gründungen in den ersten Jahren überlebensfähiger sind als eine vergleichbare Gruppe von Gründungen aus den gleichen Jahren. 
Die folgende Graphik zeigt die Überlebensraten der nunmehr über mehrere Jahre beobachtbaren $A p$ lusB-Gründungskohorte von 2007. Die im Jahr 2007 gegründeten AplusB-Unternehmen traten zu unterschiedlichen Zeitpunkten von 2004 bis 2006 in die AplusB-Zentren ein. Die für die Kontrollgruppe ermittelte Überlebensrate wurde hinsichtlich der Branchenstruktur bereinigt, was einen direkten Vergleich unterstützt.

Es zeigt sich, dass die AplusB-Gründungen eine deutlich höhere Überlebensrate aufweisen als die Vergleichsunternehmen des gleichen Gründungsjahres. Bemerkenswert ist, dass sich die Zahl der überlebenden Unternehmen nach mehreren Jahren sogar stabilisiert.

Abbildung 29 Überlebensraten der AplusB-Gründungen im Jahr 2007 nach drei Jahren im Vergleich zu einer branchenstrukturbereinigten Kontrollgruppe, in \% der Gründungsfälle

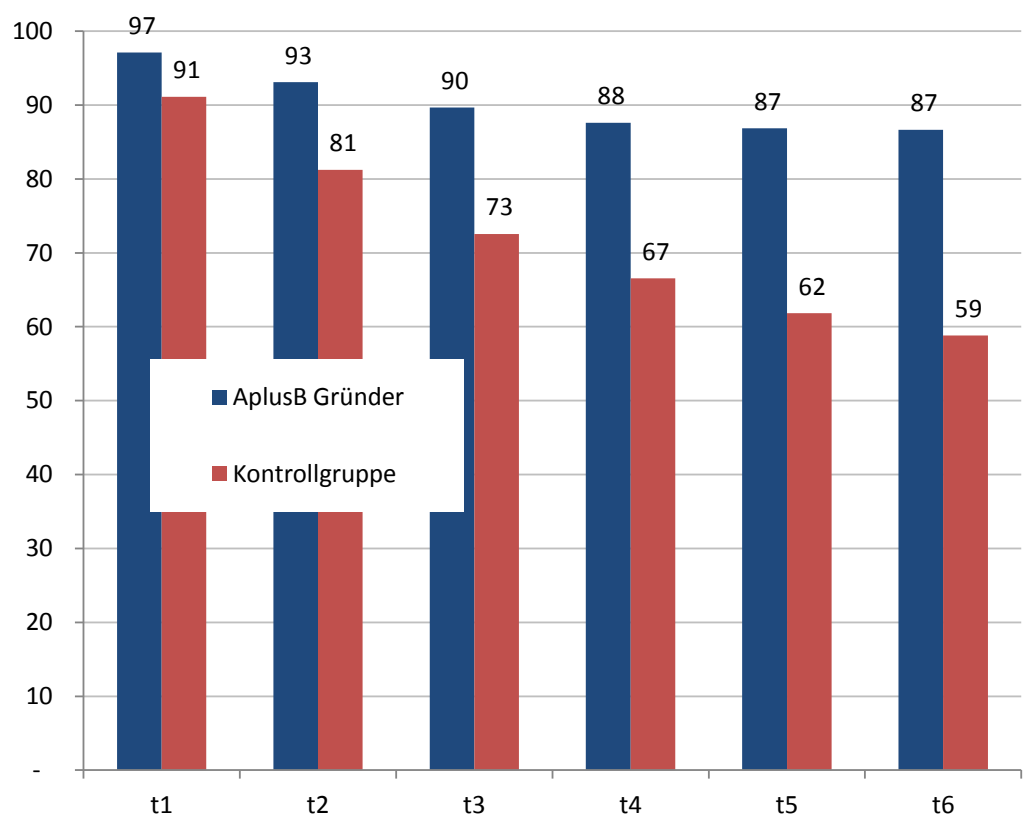

Quelle: Statistik Austria, Berechnungen JR

Dies erklärt sich durch eine bessere Vorbereitung auf die Entscheidung, zu gründen, sowie auch auf die Gründung selbst. Verfolgt man die einzelnen AplusB-Fälle in der Statistik, so wird deutlich, dass die Überlebensraten der Unternehmen sogar geringfügig höher sind als die ermittelten Zahlen.

Der Grund besteht darin, dass einzelne Unternehmen aus der Gruppe der AplusB-Gründungen, aber auch der Kontrollgruppe, nach der Gründung Phasen aufweisen, in denen die Geschäftstätigkeit mehr als ein Jahr auf „Null“ heruntergefahren wird, womit die Statistik von einer Stilllegung (ruhendes Gewerbe) ausgeht.

Die nachstehende Graphik zeigt den Anteil überlebender Unternehmen nach drei Jahren von vier aufeinanderfolgenden Gründungskohorten. Dabei ist ein klarer Trend zur Verkürzung der Überlebensfähigkeit von Gründungen festzustellen. Dies erschwert, neben der eingeschränkten Verfügbarkeit langer Zeitreihen, eine aggregierte Betrachtung der längerfristigen Überlebensfähigkeit. Ab dem Gründungsjahr 2007 basiert die Gründungs- und Überlebensstatistik auf einer Einzelverfolgung von Unternehmen. Dies wird in Zukunft auch langfristige Untersuchungen erlauben, die nachvollziehen können, inwieweit diese Entwicklungen durch die Veränderungen im Zusammenhang mit der jüngsten Rezession zusammenhängen. 
Abbildung 30 Überlebensraten österreichischer Gründungen (in \% der Gründungsfälle) im Jahr 2008 nach drei Jahren (2011) in unterschiedlichen Branchenbereichen (lt. OECD Klassifikation)

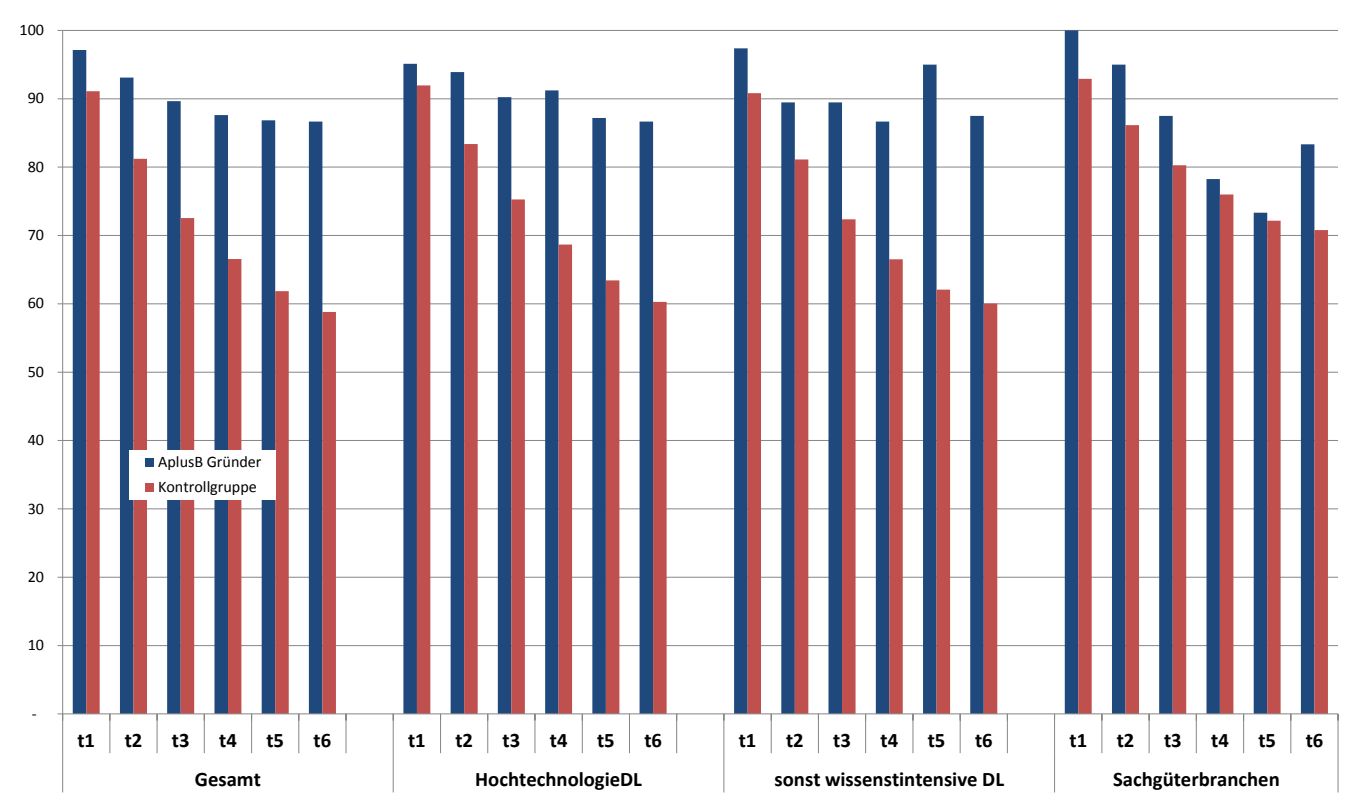

Quelle: Statistik Austria, JR-Berechnungen

Im Bereich der Hochtechnologie war in den drei Jahren vor der Wirtschaftskrise sogar ein Anstieg der Überlebensraten beobachtbar. Die folgende Darstellung zeigt, dass sich die für die AplusB-Gründungen und die Kontrollgruppe wahrgenommene Veränderung der Überlebensraten einem über viele Branchenbereiche hinweg beobachtbaren Trend entspricht, der durch die Krisenjahre mehr verstärkt als ausgelöst wurde.

Abbildung 31 Langfristige Entwicklung der Überlebensraten österreichischer Gründungen (in \% der Gründungsfälle) nach drei Jahren (2011), in unterschiedlichen Branchenbereichen (lt. OECD Klassifikation)

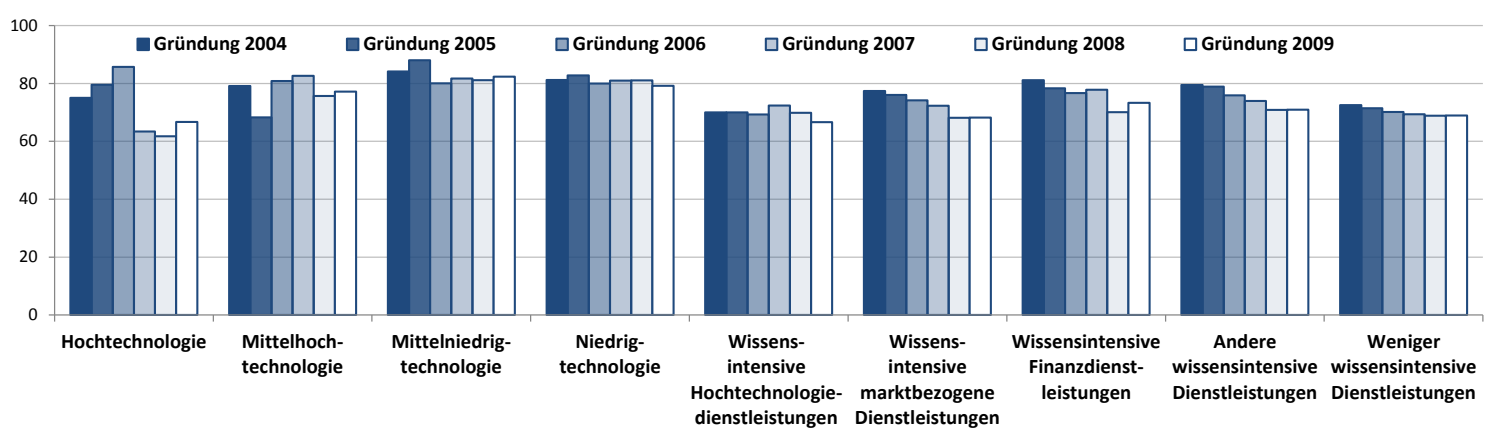

Quelle: Statistik Austria, JR-Berechnungen

Neben der Überlebensrate der unterstützten Gründungen stellt die Beschäftigungsentwicklung der jungen Unternehmen in den ersten drei Jahren nach der Gründung ein wichtiges Indiz für die Wirkungen des Programms dar.

Während durchwegs regionale Unterschiede in der Gründungsneigung festzustellen sind, schlagen sich regional unterschiedliche Gegebenheiten sowohl bei den AplusB-Gründungen als auch bei der Kontrollgruppe nur unwesentlich in der Überlebensfähigkeit der gegründeten Unternehmen nieder. 
Abbildung 32 Überlebensraten von Gründungen im Jahr 2008 (in \% der Gründungsfälle) nach drei Jahren (2011), in ausgewählten Bundesländern

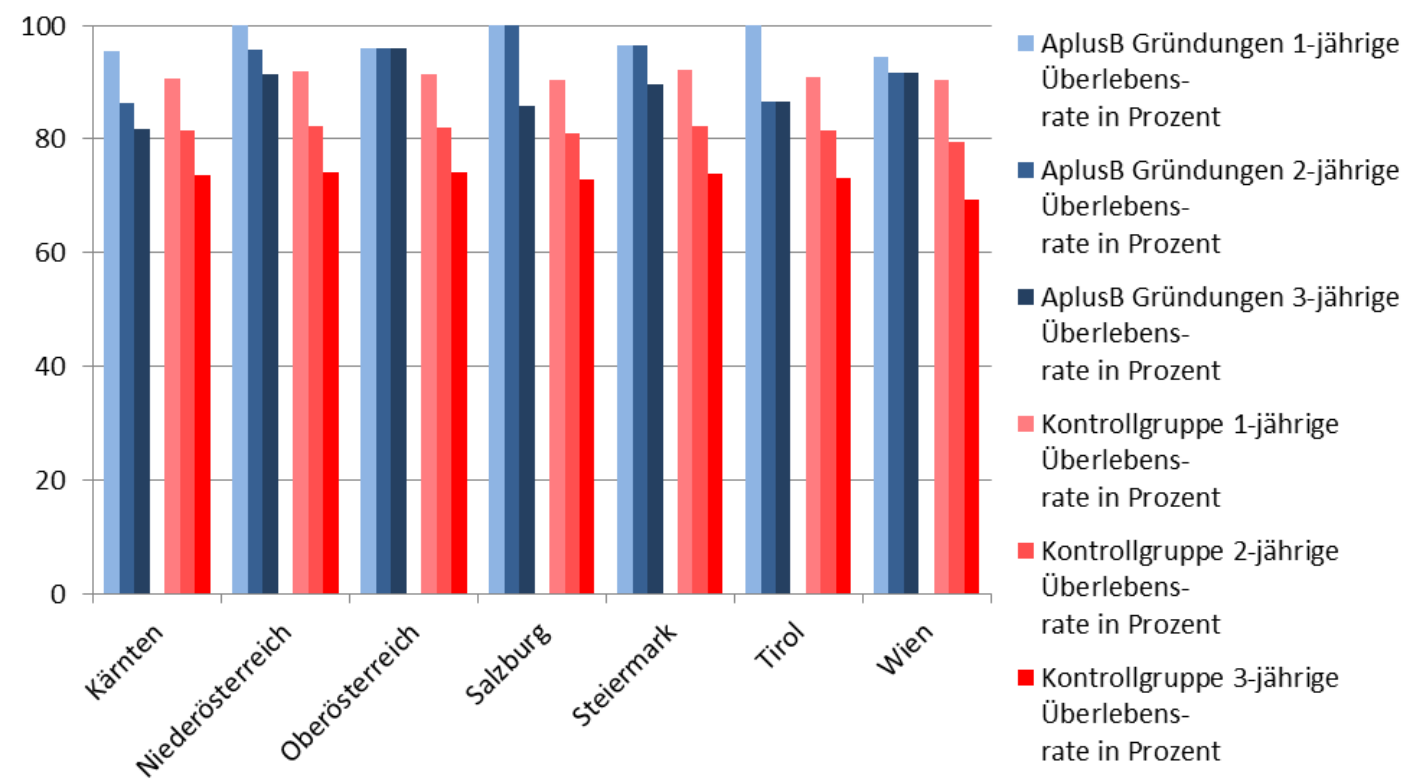

Quelle: Statistik Austria, JR-Berechnungen

\section{Die Beschäftigungsentwicklung von AplusB-Gründungen im Vergleich}

An dieser Stelle ist zu bemerken, dass die Gründungsstatistik grundsätzlich auch Großgründungen, bspw. Spin-offs aus größeren Unternehmen als Gründung anerkennen. Soweit diese Gründungen in die Kontrollgruppe einbezogen werden, verzerren sie die Ergebnisse der kontrafaktischen Betrachtung. Aus diesem Grund wurde die Kontrollgruppe um jene Gründungen bereinigt, die bereits bei der Gründungen mehr als zehn Beschäftigte oder mehr als zwei Mio. $€$ Umsatz aufwiesen.

Abbildung 33 Beschäftigungsentwicklung der Gründungskohorten ab 2007 in den ersten sieben Jahren nach der Gründung ${ }^{49}$

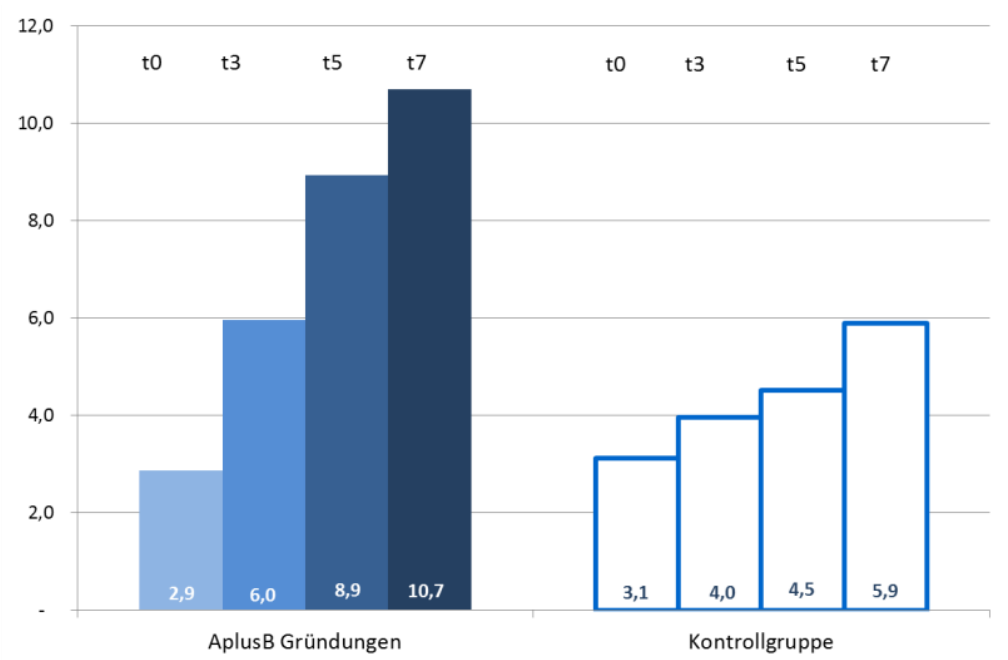

Quelle: Statistik Austria, JR-Berechnungen

${ }^{49}$ Der Vergleich bezieht sich auf Unternehmen mit einem Jahresumsatz von mindestens $30.000 €$. 
Wie anhand von Abbildung 33 deutlich wird, unterscheiden sich die AplusB-Gründungen im Jahr der Gründung hinsichtlich der Zahl der Beschäftigten nicht von jenen der Kontrollgruppe. Die AplusBUnternehmen weisen allerdings in den darauf folgenden Jahren eine deutlich dynamischere Entwicklung auf als die Kontrollgruppe. Im siebten Jahr nach der Gründung (t7), d.h. in der Regel neun Jahre nach der Aufnahme in das AplusB-Zentrum, weisen die überlebenden AplusB-Fälle nahezu doppelt so viele Beschäftigte auf wie die überlebenden Unternehmen der Kontrollgruppe.

Das Bild wird maßgeblich von dem bei AplusB dominierenden Bereich der HochtechnologieDienstleistungen geprägt. Für die bei AplusB entwickelten Gründungen im Sachgüterbereich kann eine günstigere Beschäftigungsentwicklung nicht mehr statistisch belegt werden. Auch wenn die Fallzahlen bei den AplusB-Gründungen im Sachgüterbereich für eine statistisch fundierte Aussage zu gering sind, ist festzustellen, dass die beobachtbaren AplusB-Fälle eine weniger dynamische Beschäftigungsentwicklung aufwiesen als die Kontrollgruppe. ${ }^{50}$

Abbildung 34 Beschäftigungsentwicklung der Gründungskohorten ab 2007 in den ersten sieben Jahren nach der Gründung

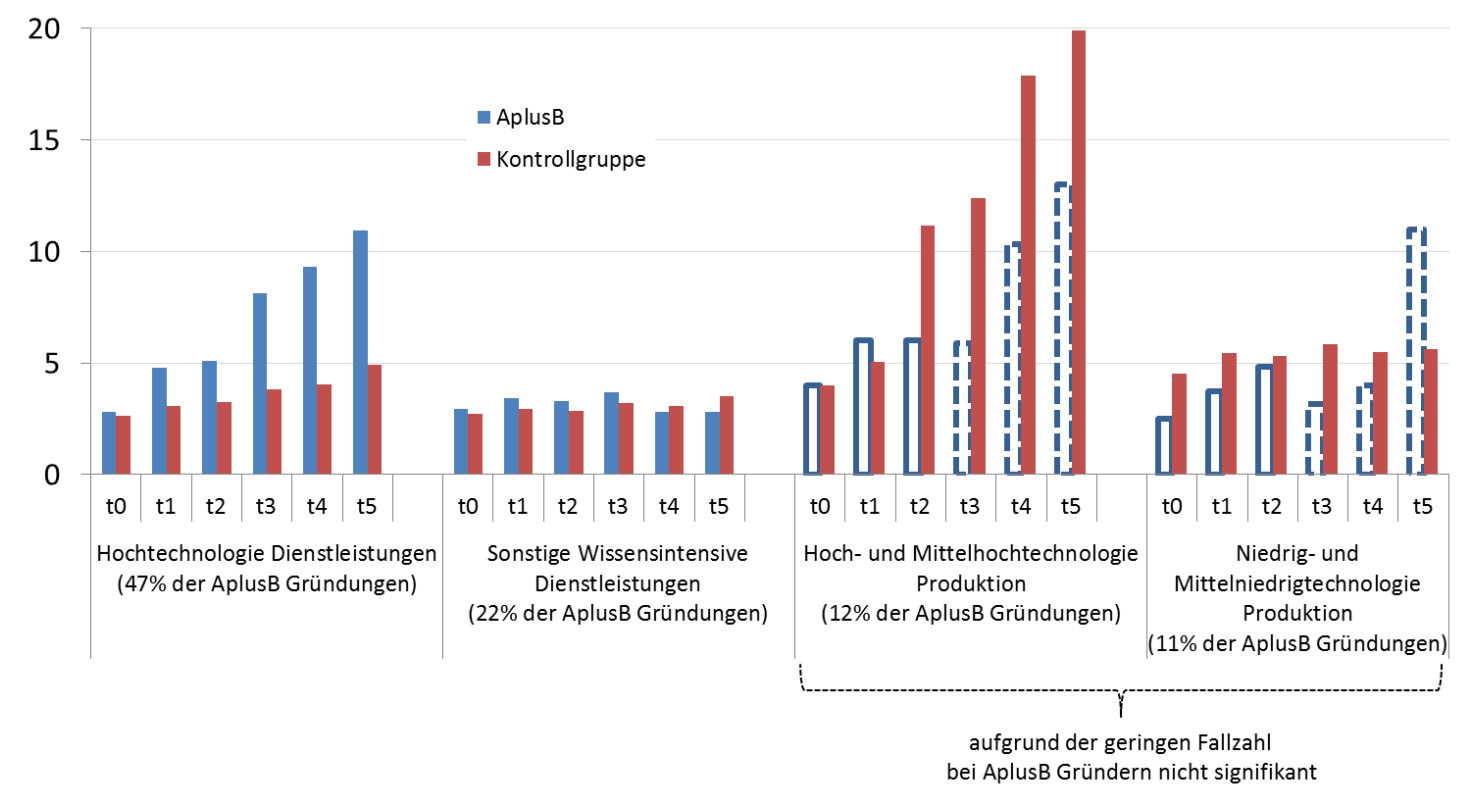

Quelle: Statistik Austria, JR-Berechnungen

Unternehmensgründungen haben eine positive Beschäftigungswirkung. In Abhängigkeit vom Überleben des Unternehmens ist diese möglicherweise nur temporal. Es stellt sich daher die Frage, inwieweit es den überlebenden Unternehmen gelingt, den Wegfall von Beschäftigung in nach kurzer Zeit wieder stillgelegten Unternehmen zu kompensieren.

Betrachtet man die Beschäftigungswirkung von einzelnen Gründungskohorten im Vergleich, so weisen einzelne Branchenbereiche sehr unterschiedliche Verläufe auf (siehe Abbildung 34). Dabei wird dargestellt, inwieweit die zum Zeitpunkt der Gründung registrierten Beschäftigten (selbständig und unselbständig) gehalten werden können und inwieweit sogar zusätzliche Beschäftigte eingestellt werden.

50 Die angestellten längerfristigen Betrachtungen können nur auf Daten für wenige Gründungskohorten zurückgreifen. Die Zahl der AplusB-Fälle im Bereich der Sachgüterproduktion ist daher für eine statistisch valide Aussage zu gering. 
Abbildung 35 zeigt die Entwicklungen in den ersten drei Jahren nach der Gründung, ausgehend vom Beschäftigungsaufkommen der betrachteten Kohorte im Jahr der Gründung (Indexwert = 100).

Im Vergleich der relevanten Branchenbereiche entwickelt sich die durch die AplusB-Gründungen geschaffene Beschäftigung sehr positiv und besser als in der Kontrollgruppe. Diese sind insbesonders im Bereich der Hochtechnologie-Dienstleistungen, dem knapp die Hälfte aller AplusB-Gründungen zuzuordnen ist.

Abbildung 35 Entwicklung der durch Gründungen (in \% der Gründungsfälle) geschaffenen Beschäftigung bis zum dritten Jahr (Indexwert: Beschäftigte im Jahr t0 :=100)

\section{AplusB Gründungen (2007-2010)}

Gründungen in HochtechnologieDienstleistungsbranchen
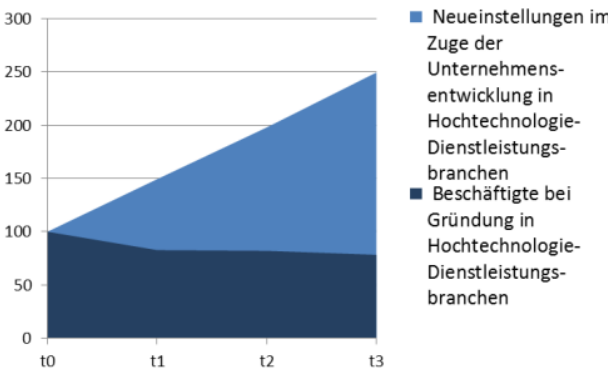

Gründungen in sonstigen wissensintensiven Dienstleistungsbranchen
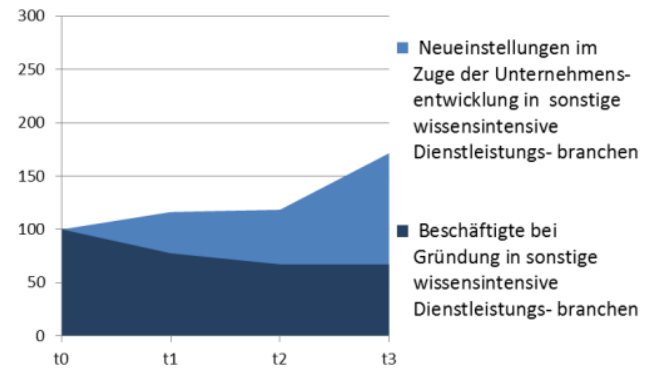

Gründungen in Hoch- u. Mittelhochtechologie Branchen
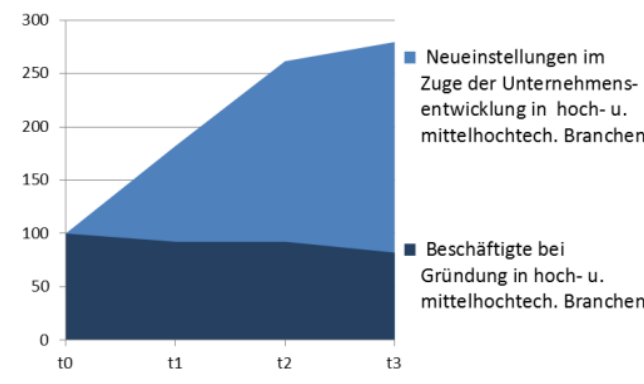

Gründungen in Niedrig- u. Mittelniedrigtechologie Branchen

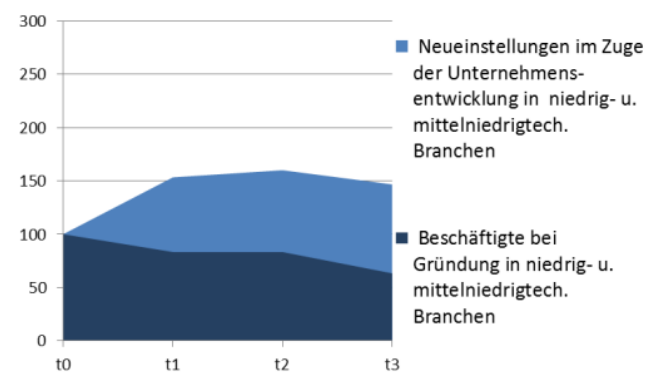

\section{Gründungen Kontrollgruppe (2007-2010)}

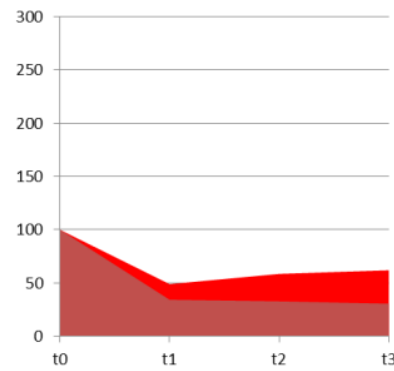

Neueinstellungen im Zuge der Unternehmensentwicklung in HochtechnologieDienstleistungsbranchen

- Beschäftigte bei Gründung in HochtechnologieDienstleistungsbranchen
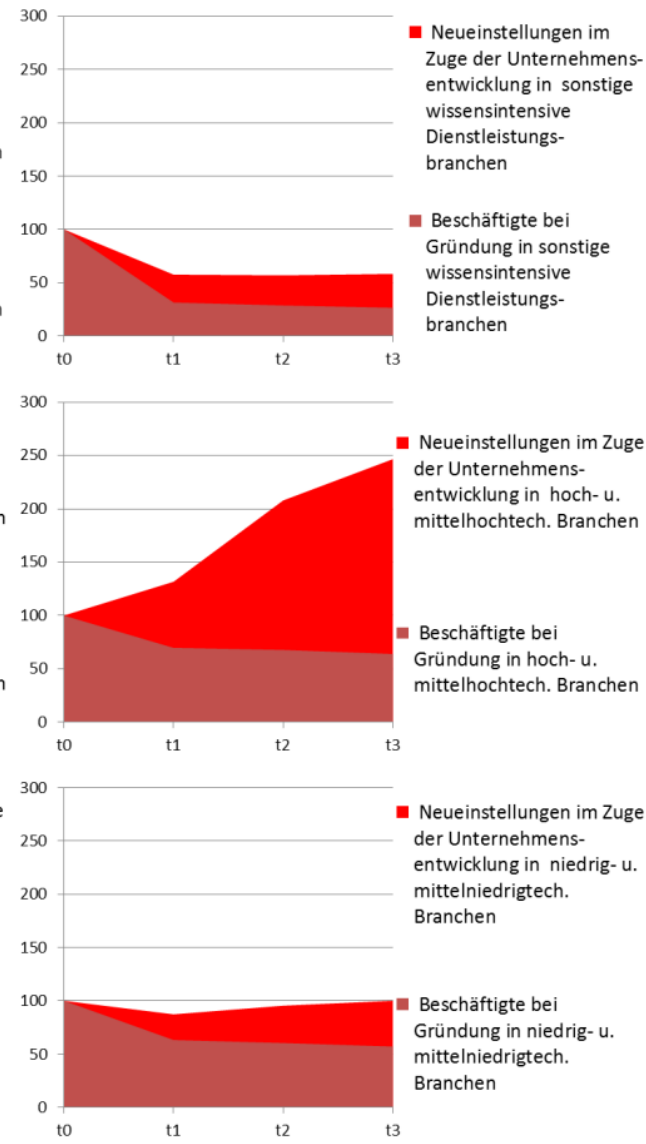

Quelle: Statistik Austria, JR-Berechnungen

Während die AplusB-Gründungen in Summe einen stetigen Anstieg bei den Beschäftigten aufweisen, kann in der Kontrollgruppe die im Zusammenhang mit der Gründung geschaffene Beschäftigung (u.a. durch Stilllegungen) nicht gehalten werden. Ähnlich positiv heben sich die AplusB-Gründungen im Bereich der sonstigen Dienstleistungen ab. 
Im Bereich der Hoch- und Mittelhochtechnologie, dem nur rd. ein Zehntel der AplusB-Gründungen zuzuordnen sind, heben sich die AplusB-Gründungen hinsichtlich der Entwicklung der Gesamtbeschäftigung in den ersten drei Jahren weniger von der Kontrollgruppe ab als in den anderen Branchenbereichen.

Zwischen 2002 und 2015 wurden lt. Eigenerhebung der AplusB-Zentren zumindest 3.400 Arbeitsplätze geschaffen. Diese Anzahl beinhaltet sowohl Abschätzungen für übernommene Unternehmen und Beschäftigte auf internationaler Ebene als auch vereinzelt Werkvertragsnehmer und einbezogene Non$A p l u s B$-Beschäftigte. Etwaige Beschäftigungsrückgänge wurden nur eingeschränkt berücksichtigt.

In direktem Abgleich mit der offiziellen Beschäftigungsstatistik zeigt sich, dass sich der Stand der Arbeitsplätze in unabhängigen AplusB-Unternehmen in Österreich bis inkl. 2013 auf 1.752 belief, davon waren 1.665 unselbstständig beschäftigt (dies entspricht 950 Beschäftigten in aktiven AplusBUnternehmen im Jahr 2010 und 382 Beschäftigten im Jahr 2007).

Abbildung 36 Summe der Beschäftigten in Österreich jeweils aller (noch) aktiven und (noch) unabhängigen AplusB-Unternehmen in den Jahren 2007-2013

\begin{tabular}{|c|c|c|c|c|c|c|c|}
\hline $\begin{array}{l}\text { Summe aller (noch) aktiven AplusB Un- } \\
\text { ternehmen im Bezugsjahr }\end{array}$ & 2007 & 2008 & 2009 & 2010 & 2011 & 2012 & 2013 \\
\hline $\begin{array}{l}\text { Anzahl aktiven AplusB Unternehmen im } \\
\text { Bezugsjahr }\end{array}$ & 127 & 175 & 219 & 272 & 311 & 344 & 386 \\
\hline $\begin{array}{l}\text { Anzahl Beschäftigte bei aktiven AplusB } \\
\text { Unternehmen in Österreich }\end{array}$ & 382 & 593 & 744 & 950 & 1.193 & 1.469 & 1.752 \\
\hline $\begin{array}{l}\text { Anzahl unselbständig Beschäftigte bei } \\
\text { aktiven AplusB Unternehmen in Öster- } \\
\text { reich }\end{array}$ & 353 & 554 & 696 & 889 & 1.121 & 1.391 & 1.665 \\
\hline
\end{tabular}

Quelle: Statistik Austria, JR-Berechnungen 


\section{Umsatzentwicklung und Exportneigung von AplusB-Gründungen im Vergleich}

Abbildung 37 zeigt die Umsatzentwicklung der überlebenden AplusB-Gründungen im Vergleich zur Kontrollgruppe. Dabei zeigt sich, dass die überlebenden AplusB-Gründungen hinsichtlich des generierten Umsatzes anfänglich deutlich hinter der Kontrollgruppe zurückliegen, diese aber nach dem überstandenen vierten Jahr sogar überholen.

Abbildung 37 Umsatzentwicklung der Gründungskohorten ab 2007 in den ersten sieben Jahren nach der Gründung (in Tsd. €)

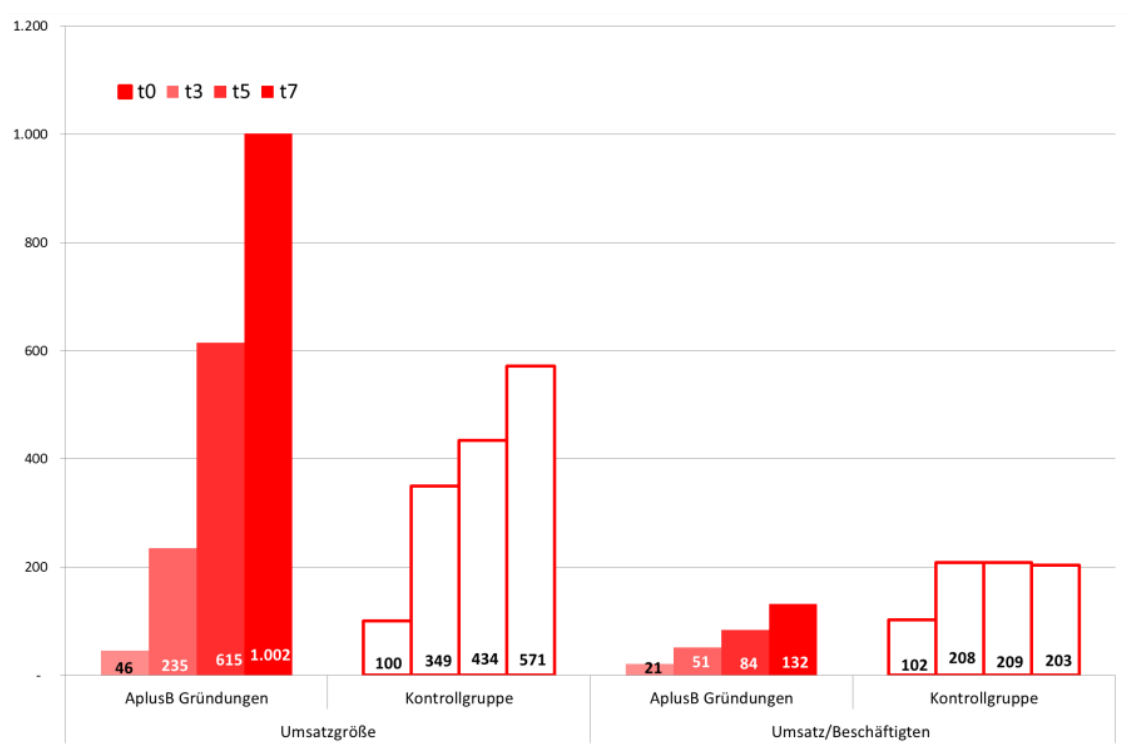

Quelle: Statistik Austria, JR-Berechnungen

Die höhere Beschäftigung pro Unternehmen schlägt sich in einem etwas geringeren Umsatz pro Beschäftigen nieder. Eine mögliche Erklärung hierfür wäre ein etwas höherer Anteil von Handelsaktivitäten bzw. zugekauften Vorleistungen im Bereich der Kontrollgruppe.

Neben der Wirkung auf die Erneuerung des Unternehmensbestandes sowie Wachstum und Beschäftigung zielt das AplusB-Programm im Besonderen auf die Steigerung der internationalen Wettbewerbsfähigkeit ab. Vor diesem Hintergrund ist von besonderem Interesse, inwieweit AplusB-Gründungen im Export aktiv sind und sich diesbezüglich von der definierten Kontrollgruppe abheben.

Abbildung 38 zeigt, dass die Exportneigung, d.h. der Anteil der exportierenden Unternehmen unter den Überlebenden der beiden Vergleichsgruppen bei den AplusB-Gründungen deutlich höher ist. Im siebenden Jahr nach der Gründung (d.h. in der Regel im neunten Jahr nach der Inkubation) sind 58 \% der überlebenden $A p l u s B$-Gründungen im Export aktiv. Bei den bis zum siebten Jahr aktiven Unternehmen in der Kontrollgruppe trifft dies auf $21 \% \mathrm{zu}$.

Jene AplusB-Unternehmen, die exportaktiv sind, wachsen stärker und generieren einen wesentlichen Teil ihres Umsatzes im internationalen Umfeld. Aufgrund dessen ergibt sich ein durchschnittlicher Anteil des Exportumsatzes am Gesamtumsatz der bis zum siebten Jahr überlebenden AplusBUnternehmen von $53 \%$. 
Abbildung 38 Exportneigung und -intensität (in \%) der Gründungskohorten ab 2007 in den ersten sieben Jahren nach der Gründung

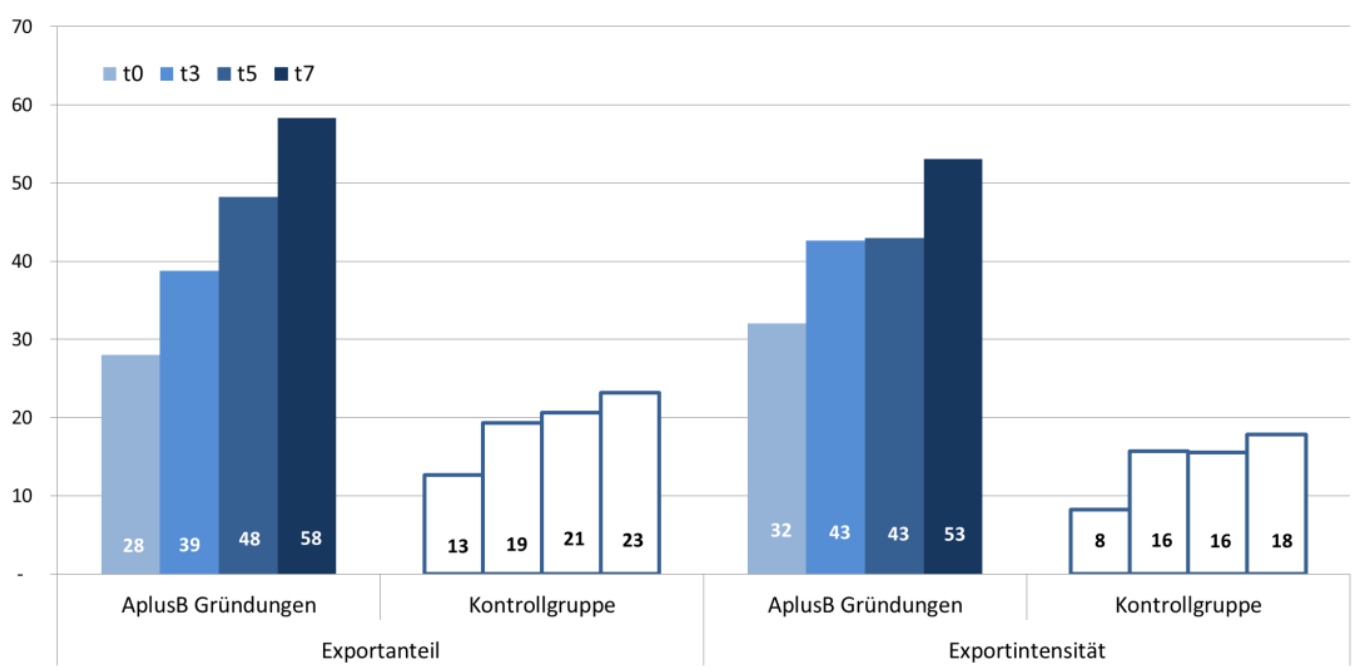

Quelle: Statistik Austria, JR-Berechnungen

\section{Forschungs- und Innovationsneigung von AplusB-Gründungen im Vergleich}

Das AplusB-Programm soll neben anderen Instrumenten einen fokussierten Beitrag zur Positionierung Österreichs in der Innovationslandschaft sowie zum langfristigen strukturellen Wandel leisten. Insofern ist es von großer Bedeutung, inwieweit AplusB-Gründungen tatsächlich eine höhere Forschungs- und Innovationsneigung aufweisen als vergleichbare Gründungen in Österreich.

Abbildung 39 Forschungsneigung und -intensität (in \%) der Gründungskohorten ab 2007 in den ersten drei Jahren nach der Gründung

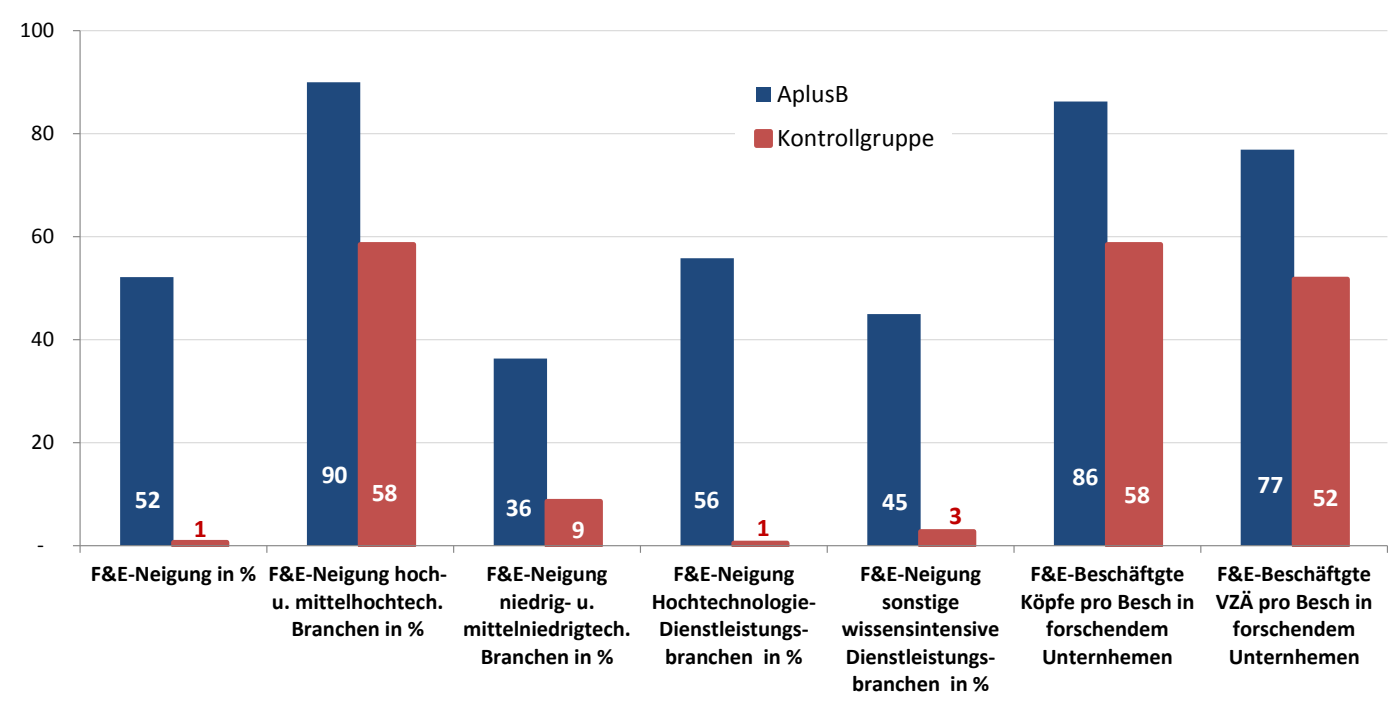

Quelle: Statistik Austria, JR-Berechnungen 
In weiterer Folge, wie auch die Zahlen zur Inanspruchnahme einschlägiger FTI-Förderungen bestätigen, heben sich die AplusB-Gründungen signifikant positiv von der betrachteten Kontrollgruppe ab. ${ }^{51}$ Mehr als die Hälfte der AplusB-Gründungen weist bis zum fünften Jahr F\&E-Aktivitäten auf, die im Rahmen der offiziellen Erhebungen der Statistik Austria registriert werden konnten. Dabei ist festzustellen, dass Gründungen im Bereich der Technologie-intensiven Sachgüterproduktion eine deutlich höhere Forschungsneigung aufweisen als jene in den Dienstleistungsbranchen. Es zeigt sich, dass die beobachteten Technologie-intensiven Sachgüterunternehmen in der Kontrollgruppe ebenfalls eine hohe F\&E-Neigung aufweisen (die allerdings deutlich unter jener der AplusB-Gründungen liegt).

Sofern junge Unternehmen forschungsaktiv sind, prägt dies einen wesentlichen Teil ihrer Aktivitäten, wie die Anteile der F\&E-Beschäftigten an der Gesamtbeschäftigung der betroffenen Unternehmen verdeutlichen (siehe Abbildung 39).

Die Wirkungen des AplusB-Programms auf die Forschungs- und Innovationslandschaft werden noch einmal deutlich, wenn man den durchschnittlichen Anteil der AplusB-Gründungen an den Gründungskohorten 2007 bis 2010 den Anteilen an den F\&E-betreibenden Unternehmen oder auch F\&E Beschäftigten in denselben Gründungskohorten gegenüberstellt (siehe Abbildung 40).

Abbildung 40 Gegenüberstellung des Anteils der AplusB-Unternehmen an den Gründungskohorten 2007 bis 2010 und der Anteile am Forschungsgeschehen im Bereich dieser Gründungskohorten

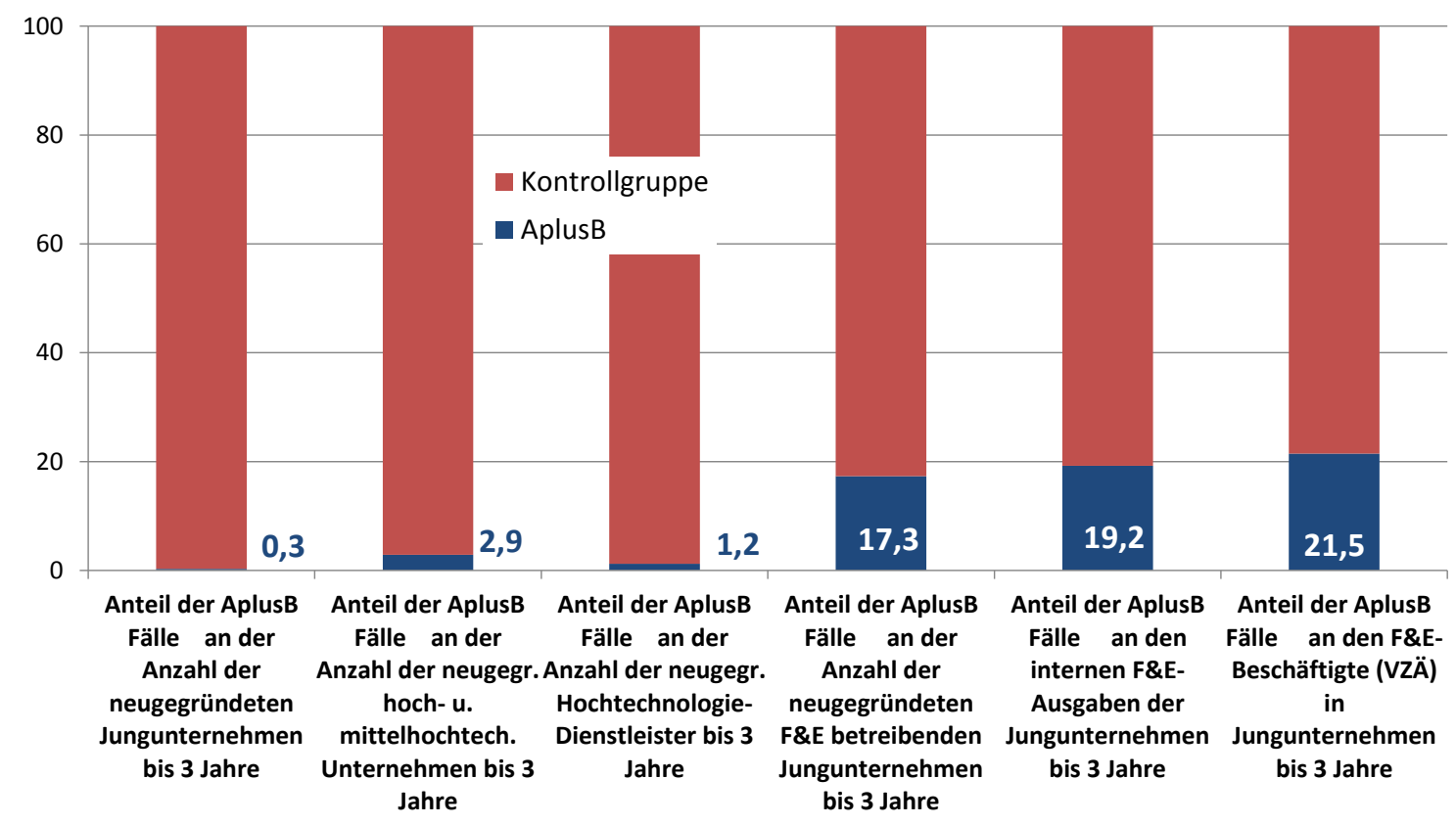

Quelle: Statistik Austria, JR-Berechnungen

\subsubsection{Die Inanspruchnahme komplementärer Förderung durch AplusB-Gründungen - Verortung in der Förderungslandschaft}

Im vorigen Abschnitt wurde die überdurchschnittliche Beteiligung von AplusB-Gründungen am Forschungs- und Innovationsgeschehen deutlich. Daran anknüpfend ist von Interesse, inwieweit

51 Auch hier wurden Unternehmen, die bereits bei der Gründung mehr als zehn Beschäftigte bzw. mehr als zwei Mio. € Umsatz aufwiesen, nicht in die Kontrollgruppe einbezogen, da es sich hier mit großer Wahrscheinlichkeit um Ausgründungen aus größeren Unternehmen handelt (bspw.um eine ausgegründete Abteilung/ Geschäftsfeld). 
sich AplusB-Gründungen auch im Rahmen der F\&E und Innovationsförderung bzw. der hier angewandten Auswahlmechanismen erfolgreich positionieren können. Für die beiden Förderagenturen aws und FFG war es möglich eine anonymisierte Abfrage auf Einzeldatenebene durchzuführen.

Abbildung 41 Inanspruchnahme von Förderungen der aws und FFG durch die AplusB-Gründungen

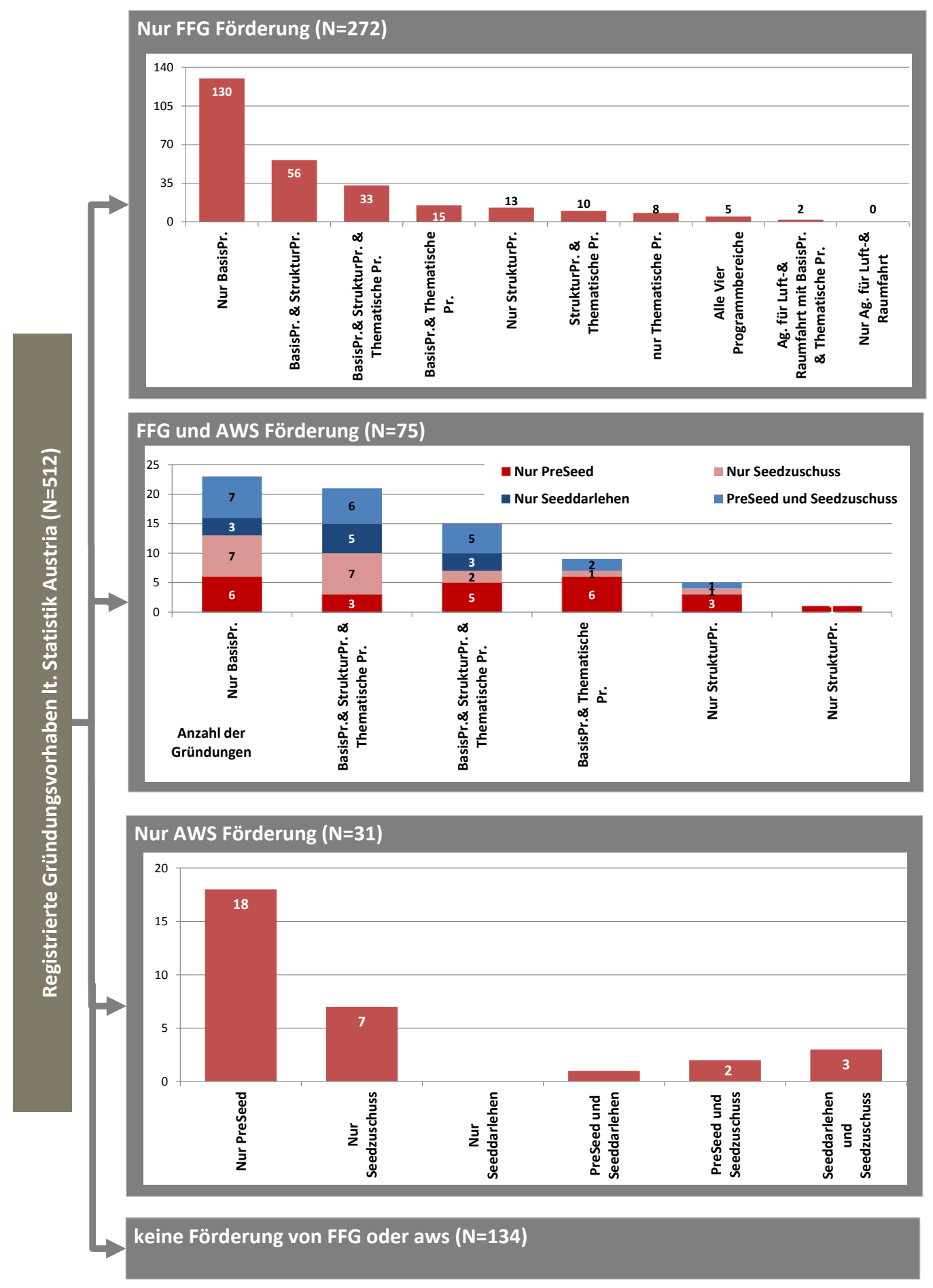

Quelle: Statistik Austria, aws, FFG, JR-Berechnungen 
Bereits die Fallstudien und die durchgeführten Interviews mit GründerInnen haben verdeutlicht, dass $A p l u s B$-Gründungen durch die Arbeit der Zentren auf weitere Fördermöglichkeiten aufmerksam werden und in der Vorbereitung zum Förderantrag unterstützt werden. Umgekehrt kam auch zum Ausdruck, dass andere Förderagenturen die Arbeit von AplusB anerkennen und potentielle Betreuungsfälle auf das Programm AplusB aufmerksam machen.

Abbildung 41 zeigt die Inanspruchnahme von Förderungen aws und FFG durch die identifizierten AplusB-Gründungen im Überblick. Dabei wird deutlich, dass die überwiegende Mehrheit (74 \%) der AplusB-Gründungen darin erfolgreich war, nach der Gründung Förderungen bei aws- oder FFGProgrammen einzuwerben. Zwei Drittel gelang es, eine FFG-Förderung erfolgreich einzuwerben. $15 \%$ der AplusB-Gründungen gelang es, sowohl bei aws als auch bei FFG-Programmen erfolgreich Förderungsmittel einzuwerben.

Vergleicht man die gesamten, von AplusB-Gründungen und sonstigen Gründungen aus der Kontrollgruppe bei der FFG eingeworbenen Förderungsmittel, wird deutlich, dass AplusB-Gründungen nicht nur eine höhere F\&E-Neigung aufweisen, sondern dass die forschenden AplusB-Gründungen auch aktiver bzw. erfolgreicher in der Einwerbung von Forschungs- und Innovationsförderungen sind.

Abbildung 42 zeigt die genehmigte Fördersumme gestaffelt nach dem Unternehmensalter. Es bestätigt sich dabei, dass die AplusB-Gründungen in der Regel schon zu einem früheren Zeitpunkt in der Lage sind, erfolgreich Forschungs- und Innovationsförderung einzuwerben.

Abbildung 42 Gesamte an identifizierte AplusB-Gründungen sowie an Gründungen aus der Kontrollgruppe (2003 bis 2013) vergebene FFG-Förderungsmittel

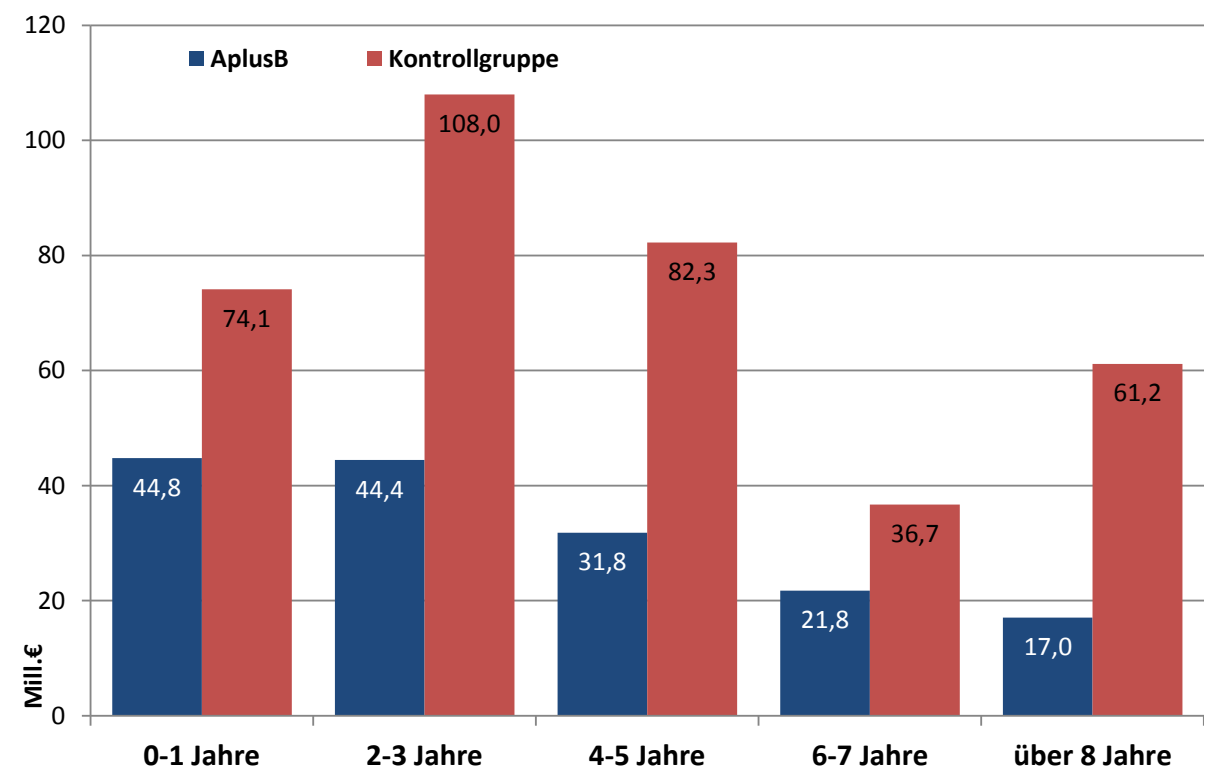

Quelle: Statistik Austria, FFG, JR-Berechnungen

Auf die einzelnen Gründungen heruntergebrochen wird deutlich, dass AplusB-Gründungen in der Lage waren, eine größere Anzahl von Projekten einzuwerben. Jene Unternehmen aus der Kontrollgruppe, die in der Lage waren, FFG-Förderungen einzuwerben, taten dies allerdings, gemessen an der Fördersumme, in ähnlichem Umfang (siehe Abbildung 43). 
Abbildung 43 Bewilligte Fördersumme bzw. geförderte Projekte pro durch die FFG geförderter Gründung

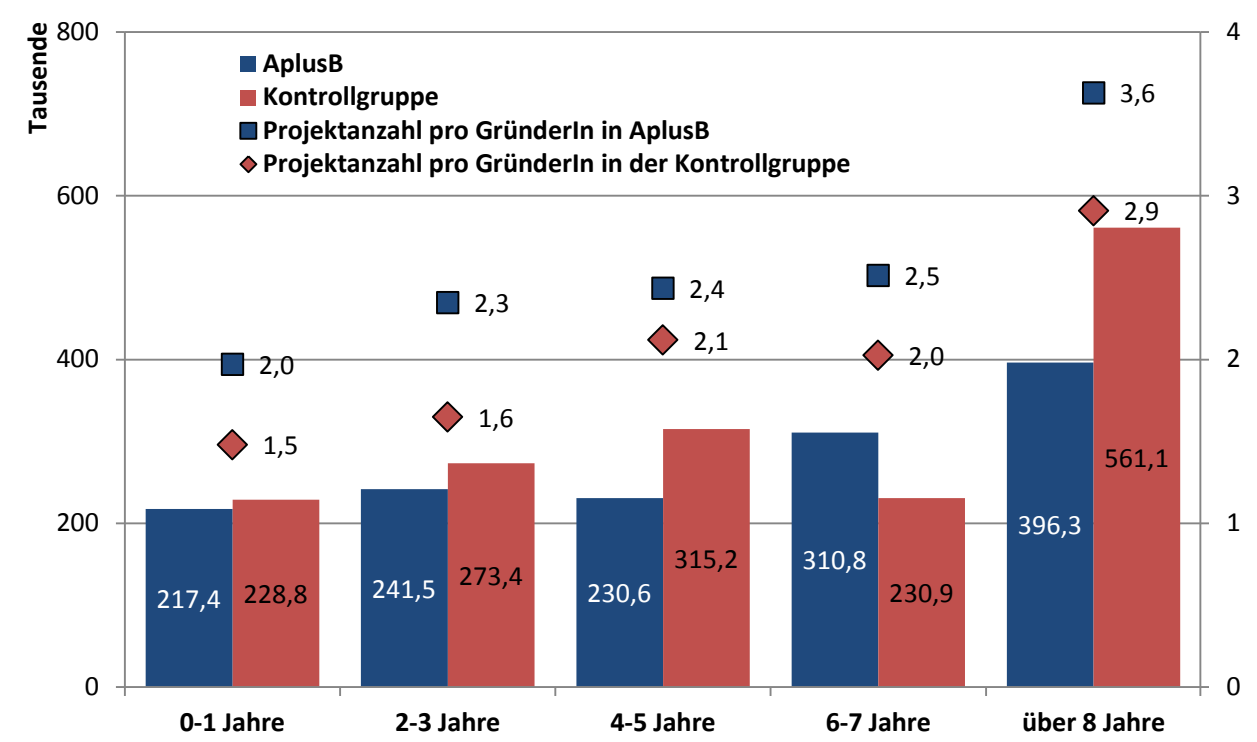

Quelle: Statistik Austria, FFG, JR-Berechnungen

\subsection{Beurteilung in Hinblick auf die Angemessenheit und den Mehrwert der er- brachten AplusB Leistungen sowie die statistisch nachvollziehbaren ökonomi- schen Wirkungen}

Wie die Fallstudienanalyse zeigt, entstehen Gründungs-Ideen aus unterschiedlichsten Motiven, Absichten bzw. Erwartungshaltungen. AplusB GründerInnen bringen in der Regel wissenschaftliche oder berufliche Erfahrungen sowie entsprechendes Selbstbewusstsein mit, weisen aber häufig in relevanten Punkten Defizite auf, an denen begleitend gearbeitet werden muss. Als Herausforderungen im Gründungsprozess sowie der betrieblichen Entwicklung wird der Weg von Ideen zu vermarktbaren Leistungen (definierten Zielgruppen und Märkten) gesehen. Dies ist kein linearer Prozess. Die Geschäftsmodelle der Gründungen entwickeln sich schrittweise und bedürfen wiederholter Adaptierungen. Lücken, etwa welche Ressource oder welches Know-how fehlt, werden teilweise erst im operativen Geschäft ersichtlich. Dabei zeigt sich, dass im Spannungsfeld aus Fokussierung auf das Kerngeschäft und Adaptierungsbedarf, dieser Prozess in allen seinen Ausprägungen anfangs von den GründerInnen vielfach unterschätzt wird. Unterstützungsbedarf besteht daher über einen längeren Zeitraum hinweg, in dem es nicht nur guter Rezepte, sondern auch einer Hilfestellung bei Reflexion und Feedback bedarf.

Das AplusB-Programm versucht, mit seinen Zentren eine derartige Begleitung entsprechend der Bedarfe einzelner Gründungen zu ermöglichen.

- Wie wurde von den Gründungswilligen - sowohl aufgenommene und erfolgreiche als auch nicht aufgenommene bzw. abgebrochene Gründungsprojekte - das Angebot und die Unterstützung des Zentrums wahrgenommen (vor und nach der Aufnahme)? Konnten nicht aufgenommenen Gründungsvorhaben durch die Erstberatung bzw. die weitere Unter-stützung seitens des Zentrums bei ihrem Weg zur Umsetzung geholfen werden?

Sowohl die durchgeführten Fallstudien als auch Einzelinterviews bestätigen die Zufriedenheit mit dem Angebot und den Leistungen der AplusB-Zentren. Die Unternehmen hätten sich nach Einschätzung der befragten GründerInnen ohne AplusB-Unterstützung weniger gut entwickelt bzw. wären nicht gegründet worden. Dabei ist festzustellen, dass die einzelnen Zentren in der Betreuung 
durchaus unterschiedliche Wege beschreiten. Dies erklärt sich zum Teil durch die Größe und regionalen Umfeldbedingungen der Zentren.

- Die AplusB-Zentren regen eine sehr detaillierte Ausarbeitung und oftmalige Reflexion der Geschäftspläne an, die blinde Flecken aufdecken. Die Beratung in der Entwicklung des Geschäftsplans wurde explizit auch von nicht- aufgenommenen Gründungen positiv hervorgestrichen.

- Die Netzwerk- und Vermittlungsarbeit der Zentren, die den Zugang zu externer und insbesondere kompetente Expertise ermöglicht, wird als besonders wertvoll angesehen. Durch MentorInnenprogramme haben die Gründungen Zugriff auf ein breiteres Spektrum Erfahrungsträgern, die die erste Phase über längere Zeit begleiten können.

- Ebenso positiv bewertet wird die Möglichkeit des Austausches mit anderen GründerInnen, die in ähnlichen Situationen sind, und, von denen viel für das eigene Vorhaben gelernt werden kann. Die Rückmeldungen einzelner befragter GründerInnen lassen vermuten, dass der Austausch zwischen GründerInnen sogar noch ausgebaut werden könnte.

- Darüber hinaus wurden auch Schulungsangebote zu geistigem Eigentum und Markenschutz sowie das Engagement der Zentren bei der Vernetzungs- und Vermittlungsarbeit sehr positiv hervorgehoben.

Nicht alle AplusB-Gründungen bedürfen der Beherbergung im Inkubator, solange der freie Zugang möglich ist. In einzelnen Fällen, wo besondere Infrastrukturbedarfe (bspw. Labore) bestehen, können diese von den AplusB-Zentren nicht abgedeckt werden.

- Ist der durch das AplusB-Programm vorgegebene Rahmen für die wirtschaftliche Umsetzung von Know-how, welches in akademischen Einrichtungen bzw. von AkademikerInnen aus der Wirtschaft entwickelt wurde adäquat?

Das Programm ist für die wirtschaftliche Umsetzung von Know-how adäquat, da es sehr früh und bedarfsgerecht beim potentiellen Entrepreneur ansetzen kann. Dies bestätigen Interviews, Fallstudien und auch der Vergleich mit anderen Programmen. Dort, wo in der jüngeren Vergangenheit effizienzsteigernde Maßnahmen gesetzt wurden, ging dies nicht zu Lasten der Betreuungsqualität.

- Gleichzeitig ist darauf hinzuweisen, dass AplusB von Programmen, insbesondere dem Research Studios Austria Programm, begleitet wird, die die Überführung von Forschungsergebnissen in Geschäftsideen anregen. Dort, wo die ZentrumsberaterInnen an die Grenzen ihrer technisch-inhaltlichen Expertise gelangen, werden geeignete Partner von außen miteingebunden. Dies erfolgt u.a. im Rahmen des Mentoringprogramms.

○ Gründungswillige Personen aus akademischen Einrichtungen sowie Gründungswillige aus der Wirtschaft weisen in einigen Aspekten unterschiedliche Ausgangspositionen auf. Es ist festzustellen, dass vergleichbare Beispiele aus dem internationalen Umfeld etwas längere Betreuungsphasen vorsehen. Anmerkung: Eine längere Betreuung muss nicht unbedingt mit einer längeren Beherbergungsphase oder einer Erhöhung des Gesamtausmaßes der Betreuung einhergehen.

- Ist die Anbindung der Zentren an die Universitäten, Fachhochschulen und Forschungseinrichtungen eng genug? Unterstützen diese in ausreichendem Maß die Aktivitäten von AplusB, um die wirtschaftliche Umsetzung von Know-how zu ermöglichen? Gibt es Barrieren, die eine Zusammenarbeit von Universitäten, Fachhochschulen, Forschungseinrichtungen und AplusB-Zentren behindern? 
Die intensive Einbindung der Hochschulen und außeruniversitären Forschungseinrichtungen stellt ein Kernelement des AplusB-Konzepts dar. Die Hochschulen und Forschungseinrichtung haben mit AplusB die Möglichkeit, einen Schritt in Richtung einer „Entrepreneurial University“ zu gehen. Die Zusammenarbeit wird, wie Einzelgespräche gezeigt haben, positiv empfunden. Die Fokusgruppen weisen auf zwei Herausforderungen hin:

- Die Akzeptanz, dass nicht nur Akteure aus dem Kreis der Trägerorganisationen, sondern auch von anderen Forschungseinrichtungen Zugang zu AplusB-Zentren haben, ist nicht immer selbstverständlich. Dies wäre in begründeten Fällen im Interesse der Mobilität der potentiellen Gründungen zu gewährleisten.

- Die AplusB-Zentren investieren einen beachtlichen und tendenziell wachsenden Teil im Bereich der Bewusstseinsbildung und Sensibilisierung. Bei den einzelnen AplusB-Zentren bestehen noch Bedarf oder Spielräume in unterschiedlichem Ausmaß, die wissenschaftlichen Einrichtungen hier noch stärker zu verpflichten.

- Wird deren technologische Wettbewerbsfähigkeit durch AplusB verbessert?

AplusB leistet einen positiven Beitrag zur Umsetzung von technologischer Wettbewerbsfähigkeit in wirtschaftliche Wettbewerbsfähigkeit. Dies illustrieren die Zahlen betreffend die F\&E- und Exportneigung, aber auch die erfolgreiche Durchsetzung der unterstützen Gründungen im Rahmen der Auswahlverfahren der nationalen Forschungsförderung sehr anschaulich.

- Wurde durch die Entwicklung des AplusB-Programms und die Etablierung von AplusB-Zentren das identifizierte Potential an Firmengründungen richtig eingeschätzt, konnte es erhöht und die Nachhaltigkeit der Gründungen gesteigert werden? Ist das AplusB-Programm somit richtig dimensioniert und dotiert worden?

Nach einem ersten Abschöpfungseffekt in Hinblick auf interessante Projekte in den Startjahren von AplusB wurde im Laufe der Programm-Implementierung ein zunehmender Bedarf an Sensibilisierungs- und Bewusstseinsbildungs-Maßnahmen deutlich. Die AplusB-Zentren haben in Abstimmung mit den wissenschaftlichen Partnerorganisationen erfolgreich reagiert.

Ein weiterer Punkt betrifft die Abgrenzung der Zielgruppe. Die Erweiterung auf innovative wachstumsorientierte Gründungen, die nicht direkt aus dem akademischen Umfeld entstehen, hat sich als sinnvoll herausgestellt und wird, wie die durchgeführten Interviews bestätigt haben, von den akademischen Trägerorganisationen mitgetragen. Das Programm bewältigt gegenwärtig rd. 50 Gründungsvorhaben pro Jahr. Vor allem am Standort Wien übersteigt das Interesse von Seiten Gründungswilliger die möglichen Kapazitäten des Zentrums bei Weitem.

Wie Einzelgespräche und auch die Fokusgruppen gezeigt haben, planen einzelne AplusB-Zentren den Bereich nicht-akademischer innovativer und wachstumsorientierter Gründungen weiter zu forcieren. Eine komplementäre Ergänzung in diesem Bereich erlaubt Skalenvorteile. Gleichzeitig sollte das AplusB-Programm, soweit es von Bundesseite gefördert wird, die Wurzeln im Bereich der akademischen Gründungen nicht aus den Augen verlieren.

- Welche Bedeutung und welche Bekanntheit kommen den Produkten und Dienstleistungen der Zentren in der Gründungscommunity zu?

Die durchgeführten Sensibilisierungsmaßnahmen sowie auch die Beteiligung an unterschiedlichen Wettbewerben haben den Bekanntheitsgrad des AplusB-Programms stark erhöht. Im Bereich der Unterstützungslandschaft genießt das Programm weitgehend Anerkennung. Auf Seiten potentieller GründerInnen, kann nach wie vor nicht vorausgesetzt werden, dass diese selbstständig zum Ap- 
lus $B$-Zentrum finden. Ideenwettbewerbe, Internetpräsenz, Informationsveranstaltungen und andere Förder- und Unterstützungseinrichtungen sind daher wichtige Eingangskanäle.

- Sind AplusB-Gründungen mit sonstigen Gründungen vergleichbar? Wo liegen Besonderheiten? AplusB-Gründungen sind in vielen Punkten mit sonstigen Gründungen vergleichbar. Folgende Punkte differenzieren AplusB-Gründungen:

- Wenig bis keine unternehmerische Erfahrungen der GründerInnen; herausfordernder Übertritt von der wissenschaftlichen Karriere in das Unternehmertum; selbstbewusste Persönlichkeiten;

- Hoher Innovationsgrad und Risiko, verbunden mit noch häufig bestehendem technologischen Entwicklungsbedarf, relativ hoher Finanzierungs- und Infrastrukturbedarf im Frühstadium;

○ Geschäftsideen und -planungen in einem sehr frühen Stadium;

○ Unzureichende Branchenkenntnisse; mangelnde Vernetzung und Verankerung im Branchenumfeld;

○ Häufiger Bedarf, von Beginn an internationale Märkte anzusprechen.

- Welche wirtschaftlichen Effekte können durch die von AplusB-Zentren betreuten Gründungen in den Bundesländern und österreichweit erzielt werden? Gibt es einen positiven Einfluss auf das Beschäftigungswachstum durch die AplusB-Gründungen? (Geschaffene Arbeitsplätze seit Beginn des Programms.)

Im Rahmen der durchgeführten statistischen Analysen wurde deutlich, das AplusB-Gründungen eine deutlich höhere Überlebensrate (AplusB-Gründungen: 90 \% nach dem dritten Jahr, 87 \% nach dem fünften Jahr; Kontrollgruppe: $75 \%$ nach dem dritten Jahr, $64 \%$ nach dem fünften Jahr) aufweisen. Dies gilt im Besonderen für den Bereich der Hochtechnologiedienstleistungen und sonstigen wissensintensiven Dienstleistungen, die den überwiegenden Anteil der AplusB-Gründungen ausmachen.

Die Analysen zeigen, dass AplusB-Gründungen eine höhere Beschäftigungswirkung (50 \% höher als die Kontrollgruppe nach dem dritten und $80 \%$ höher als die Kontrollgruppe nach dem fünften Jahr) erzielen. Des Weiteren starten AplusB-Unternehmen mit geringerer Umsatzgröße, überholen die Kontrollgruppe aber aufgrund einer höheren Dynamik im Durchschnitt im vierten Jahr nach der Gründung. Ende 2013 waren 1.752 Personen zu diesem Zeitpunkt aktiven und unabhängigen $A p l u s B$-Unternehmen in Österreich beschäftigt. Unter Berücksichtigung von Beschäftigten in bereits aufgekauften AplusB-Unternehmen, von Beschäftigten in etwaigen Auslandsdependancen sowie von indirekter und induzierter Beschäftigung, die durch die wirtschaftliche Aktivität der $A p$ lusB-Unternehmen geschaffen wurde, ist von einer darüber deutlich hinausgehenden Beschäftigungswirkung des Programms auszugehen.

- Wäre/Ist das Unternehmen auch ohne die Unterstützung durch das AplusB-Programm gegründet worden?

Die befragten Fallstudien sowie $30 \%$ der im Rahmen des Gründungs-Monitorings befragten Unternehmen hätten das Unternehmen ohne AplusB nicht gegründet. Weitere $52 \%$ der Befragten gibt an, das Unternehmen in anderer (d.h. vorrangig in reduzierter) Form gegründet zu haben. Die Additionalität bewegt sich damit in einem ähnlichen Bereich, wie sie im Zusammenhang mit dem Wirkungsmonitoring der FFG festgestellt werden kann.

- Inwieweit wurden die Mission bzw. die einzelnen Ziele erreicht? Inwieweit tragen die Landesförderungen zur Zielerreichung bei? 
Das AplusB-Programm hat wesentliche Ziele erreicht. Hierfür sprechen die Zahl der betreuten Fälle (Gründungspotential), deren wirtschaftlicher Erfolg, aber auch die starke Präsenz der AplusBGründungen im Bereich der F\&E-Statistik im Wettbewerb um Förderungen sowie bei Start-upWettbewerben.

Ein weiterer Punkt betrifft die gestiegene und mittlerweile hohe Sensibilität und Kooperationsbereitschaft bei wissenschaftlichen Einrichtungen und regionalen Fördereinrichtungen.

In zwei Punkten sind die gegenwärtigen Berichts- und Monitoring-Grundlagen wenig geeignet, die Zielerreichung zu messen. Die betrifft im Allgemeinen Output-Indikatoren (im Besonderen im Bereich der Sensibilisierungs- und Vorfeldarbeit) sowie Beobachtung der Gründungen nach dem Austritt aus den Zentren. Auch unter Berücksichtigung des relativ niedrigen Anteils von Wissenschaftlerinnen im technisch-naturwissenschaftlichen Bereich ist die Zahl der Gründerinnen verbesserungswürdig. Auch hier erlaubt das Gründungs-Monitoring keine exakte Auswertung. 


\section{Schlussfolgerungen und Handlungsempfehlungen}

- Die Aufmerksamkeit und das Bewusstsein für die strukturpolitische Bedeutung von Entrepreneurship und wissensintensive Gründungen sind weltweit, so auch in Österreich, stark angestiegen. AplusB hat in diesem Zusammenhang zur Sensibilisierung auf nationaler bzw. regionaler Ebene, im Besonderen was Gründungen aus dem akademischen Umfeld anbelangt, einen wesentlichen Beitrag geleistet.

- Die kommerzielle Verwertung von Forschungsergebnissen rückt an Forschungseinrichtungen, speziell an Hochschulen, verstärkt in den Mittelpunkt. Themen sind hier Lizenzierung, der Verkauf von Patenten bzw. von universitärem Know-how sowie Industriekooperationen. Hochschulen sind im Bereich der „Third-Mission“ unterschiedlich intensiv engagiert. Auch wenn das Problembewusstsein und strategisches Commitment an den wissenschaftlichen Einrichtungen besteht, sind diese in ihren Ressourcen und Instrumenten, Gründungen mit dem notwendigen Erfahrungshintergrund und über einen längeren Zeitraum zu begleiten, beschränkt. Im Zusammenspiel mit AplusB können Hochschulen und Forschungseinrichtungen allerdings einen wesentlichen Beitrag im Bereich der Bewusstseinsbildung und Vorfeldarbeit übernehmen. Dies ist gegenwärtig ausbaufähig.

- AplusB-Gründungen sind üblicherweise technologieorientierte, hochkomplexe, in der Betreuung aufwendige Projekte, vor allem über einen längeren Zeitraum. Unterschiede im Aufwand lassen sich vor allem zwischen sogenannten Hard- und Softwaregründungen feststellen; letztere sind überwiegend im IKT-Bereich (IKT-Anwendungen in unterschiedlichen Sektoren) angesiedelt.

- Die grundlegenden Herausforderungen für High-Tech-Gründungen unterscheiden sich von jenen für traditionelle Gründungen weniger in der Form als in der Ausprägung. Diese haben sich zudem in den letzten Jahren nur wenig verändert: schwach ausgeprägtes Know-how von Betriebswirtschaft und Unternehmungskultur (vor allem unter akademischen GründerInnen), wenig tragfähige Businesskonzepte, mangelhafte Verwertung, fehlende Finanzierungsquellen, im HighTech-Bereich fehlendes Risikokapital, mangelnde Infrastruktur und Räumlichkeiten sowie ein hoher bürokratischer Aufwand. Die Analysen weisen jedoch darauf hin, dass eine gewisse Anzahl an AplusB-GründerInnen bereits in der Privatwirtschaft tätig war, von daher also zumindest eine gewissen Grundvorstellung von einer Unternehmensgründung und den damit einhergehenden Erfahrungen hatten.

- Den genannten Herausforderungen wird mit einem gewachsenen Angebot an öffentlichen, teilweise auch privaten Unterstützungsangeboten und Initiativen begegnet, was zuletzt zu einer gewissen Unübersichtlichkeit sowie einer Zersplitterung der Unterstützungslandschaft beigetragen hat.

- Die „Ownership“ an den verschiedenen wissenschaftlichen Einrichtungen ist unterschiedlich ausgeprägt: Einige Universitäten und Fachhochschulen sehen die „Third Mission“ als wesentlichen Eckpfeiler ihrer künftigen Entwicklung und haben bereits entsprechende Institutionen geschaffen. Andere plädieren für eine klare Rolle der Akteure im Innovationssystem und sehen die Kernaufgabe der Universitäten in der Grundlagenforschung und Lehre und nicht in der Entwicklung von Start-up-Maßnahmen. Auf Grund der zur Verfügung stehenden Ressourcen und Instrumente sind wissenschaftliche Einrichtungen nur eingeschränkt in der Lage, hoch-komplexe Gründungprojekte über einen längeren Zeitraum intensiv zu begleiten. Im Zusammenspiel mit AplusB können Hochschulen und Forschungseinrichtungen allerdings einen wesentlichen Beitrag im Bereich der Bewusstseinsbildung und Vorfeldarbeit übernehmen

- Private Anbieter können das Angebot von AplusB ergänzen, jedoch nicht im derzeitigen Umfang ersetzen oder übernehmen. Private Initiativen haben üblicherweise nicht das Ziel und sind dafür 
nicht ausgestattet, Gründungen zu stimulieren (z.B. Bewusstseinsbildung, Pre-Inkubation) oder risikoreiche und erheblich entwicklungsbedürftige Projekte in frühen Stadien zu übernehmen. Schließlich ist anzumerken, dass private Initiativen regional sehr ungleich verteilt sind.

- Die Angebote von AplusB passen sich an die regionale Situation bzw. dem Interesse der Netzwerkund Kooperationspartner an, was zu einem uneinheitlichen Angebotsprofil der einzelnen Zentren führt. Die Ausgangsbasis der Gründungsvorhaben (Erfahrung der GründerInnen, Hard/Softwaregründung) sowie der unterschiedliche Reifegrad der Gründungsideen führt zu unterschiedlich hohen Betreuungsaufwänden. Gerade deswegen ist es wichtig, „Good Practices“ untereinander auszutauschen.

- Die Zentren unterscheiden sich in Abhängigkeit von Angebotsprofil und Ausgestaltung (Größe, Zahl der Standorte) auch hinsichtlich der Kostenstruktur erheblich. In einzelnen Fällen wurden in jüngerer Zeit gezielte Maßnahmen mit dem Ziel von Effizienzsteigerungen und Kosteneinsparungen realisiert. Verglichen mit akademischen Inkubatoren in Deutschland handelt es sich bei den AplusB-Zentren um kleinere Einheiten.

AplusB trug und trägt indirekt zur Steigerung der Awareness bezüglich Selbstständigkeit bei. Gleichzeitig wird der Fokus von AplusB nicht auf der Ausräumung gründungshemmender Faktoren sowie der Motivation und Initiierung von akademischen Gründungen gesehen.

Aplus $B$ wird als starker Treiber in der Gründerszene wahrgenommen, was sich etwa dadurch zeigt, dass die erfolgreichsten Gründungen der letzten Jahre eben oft mit AplusB in Verbindung stehen. Der Verein Österreichisches Inkubatorennetzwerk AplusB ergänzt die Kommunikation nach außen, auch wenn ein vollständiger Auftritt in Englisch der internationalen Zugänglichkeit noch ein wenig im Weg steht.

- Das AplusB-Programm setzt bereits in sehr frühen Phasen mit seiner Betreuung von Gründungsvorhaben an. Anders als das FFG-Programm Research Studios Austria setzt AplusB nicht beim Forschungsprojekt und der Verwertung von dessen Ergebnissen an, sondern bei der Gründungsidee, die bereits von GründerInnen vertreten wird. Die Zentren bieten Information und Beratung in einem Umfang und einer Tiefe an, wie sie aktuell in Österreich von keinem anderen Akteur geboten werden.

- Das Programm AplusB und die Arbeit der AplusB-Zentren werden von den betreuten Gründungen sehr positiv bewertet. Die AplusB-Zentren werden als vertrauenswürdiger Partner mit Kompetenz in mehreren Themenfeldern, hoher Professionalität, Glaubwürdigkeit und Motivation und einem starken und wachsenden Netzwerk wahrgenommen, der für Unabhängigkeit ohne Eigeninteresse steht. Potentielle GründerInnen werden von Beginn an intensiv begleitet, ihre spezifischen Probleme individuell geklärt und an ihr persönliches Ziel geführt.

Rund $30 \%$ der AplusB-Gründungsvorhaben wären ohne das Programm nicht in Angriff genommen worden, weitere $52 \%$ lediglich in reduzierter Form.

Die Wirkungen des Programms sind auch statistisch nachvollziehbar:

- AplusB-Gründungen weisen eine deutlich höhere Überlebensrate (AplusBGründungen: $90 \%$ nach dem dritten Jahr, 87 \% nach dem fünften Jahr; Kontrollgruppe: $75 \%$ nach dem dritten Jahr, $64 \%$ nach dem fünften Jahr)

- Die Analysen zeigen, dass AplusB-Gründungen eine höhere Beschäftigungswirkung (50 \% höher als die Kontrollgruppe nach dem dritten und 80 \% höher als die Kontrollgruppe nach dem fünften Jahr) erzielen.

- Des Weiteren starten AplusB-Unternehmen mit geringerer Umsatzgröße, überholen die Kontrollgruppe aber aufgrund einer höheren Dynamik im Durchschnitt im vierten Jahr nach der Gründung. 
○ Mehr als die Hälfte der AplusB-Gründungen weist bis zum fünften Jahr F\&EAktivitäten auf. Trotz ihrer vergleichsweise geringen Zahl entfällt rd. ein Fünftel der F\&E-Aufwendungen und -Beschäftigten aller Jungunternehmen bis zum fünften Jahr auf AplusB-Gründungen. Ein ähnliches Bild zeigt sich im Zusammenhang mit der erfolgreichen Bewerbung bei Förderungsprogrammen.

- Die Zusammenarbeit zwischen Zentren und Netzwerk- und Kooperationspartnern wird allgemein als sehr gut eingeschätzt. Die AplusB-Plattform hat sich als mittlerweile gut frequentiertes Instrument etabliert. Sie unterstützt den Austausch zwischen den AplusB-Zentren, aber auch mit dem Programmmanagement. Die Plattform wird zunehmend für Wissenstransfer und Austausch von „Good Practices“ genutzt. Der Zentren-übergreifende Austausch zwischen GründerInnen wird gegenwärtig nicht offensiv verfolgt.

- Das AplusB-Programm verfügt über ein umfangreiches Berichts- und Monitoringsystem und war diesbezüglich anderen Förderungsprogrammen von Anbeginn an voraus. Die Evaluierungsarbeiten und damit einhergehenden Auswertungen haben allerdings auch Spielräume für Vereinfachungen und Verbesserungen deutlich gemacht.

Aus der heutigen Sicht erscheint die Weiterführung des Programms (nach einer Zwischenevaluierung 2017) unter Berücksichtigung der folgenden Empfehlungen als sinnvoll und notwendig:

- Die gemeinsame Abstimmung und Konzeption auf der nationalen Ebene in Kombination mit der Arbeit mit wissenschaftlichen Einrichtungen und Fördereinrichtungen vor Ort stellt eine Besonderheit und Stärke des Programms AplusB dar und sollte beibehalten werden. Die AplusB-Zentren stellen eher kleine, jedoch hochflexible Einheiten dar. Die mit der aktuellen Förderungsperiode bekräftigte Entscheidung, zumindest regional konzentrierte Einheiten in einer kritischen GröBe zu unterstützen, hat sich als sinnvoll herausgestellt. Dies schließt nicht aus, eine Prüfung vorzunehmen, inwieweit jene Bundesländer, die gegenwärtig über keine AplusB-Zentren verfügen, ausreichend versorgt bzw. nach Maßgabe und Klärung der rechtlichen Verhältnisse regional mitbetreut werden können.

- $\quad$ Einzelne AplusB-Zentren sehen den Bedarf, den Bereich nicht-akademischer innovativer Gründungen in Zukunft intensiver zu verfolgen, um auf regionale Potentiale noch besser reagieren zu können. Soweit es vom Bund gefördert wird, müssen auch bei der Erweiterung des Horizonts der einzelnen Zentren die Wurzeln bei „High-End“-Gründungen im Umfeld wissenschaftlicher Einrichtungen im Fokus bleiben.

- Die bilaterale Abstimmung der AplusB-Zentren mit regionalen Förderungsstellen und Stakeholdern wird in Anerkennung der sehr unterschiedlichen Herausforderungen und Bedarfe positiv bewertet. Die AplusB-Zentren müssen dementsprechend ihr Angebot anpassen (können). Das Commitment regionaler Stakeholder und Förderungsstellen schafft Planungssicherheiten. In diesem Sinn ist das Bedürfnis regionaler Förderungsstellen und Stakeholder, sich in regelmäßigen Abständen direkt mit dem Programmmanagement sowie dem bmvit abzustimmen, nachvollziehbar; der im Zusammenhang mit dem Programm COMET gewählte Ansatz könnte hier als Anregung dienen.

- Die Abstimmung zwischen den Zentren bzw. mit privaten Initiativen scheint ausbaufähig zu sein. Private Initiativen und AplusB-Zentren weisen grundsätzlich komplementäre Foki und Angebote auf. Im Zusammenspiel mit privaten Initiativen bietet sich eine klare Positionierung von AplusB, die auch national abgestimmt werden sollte.

Private Anbieter können das Angebot von AplusB ergänzen, jedoch nicht im derzeitigen Umfang 
ersetzen oder übernehmen. Private Initiativen sind nicht dazu berufen oder dafür ausgestattet, Gründungen zu stimulieren (Bewusstseinsbildung, Pre-Inkubation) oder risikoreiche und erheblich entwicklungsbedürftige Projekte in frühen Stadien zu übernehmen. Komplexe Projekte mit unklarem Entwicklungspfad wären ohne öffentliche Unterstützung und Förderung nicht oder nur schwer überlebensfähig. Schließlich ist anzumerken, dass private Initiativen regional sehr ungleich verteilt sind.

Es wird daher die Darstellung eines klaren Profils sowie die Definition von klaren Nahtstellen zu privaten Initiativen empfohlen, die nach außen kommuniziert werden.

- Die Bedarfe in Hinblick auf Bewusstseinsbildung oder auch Alumni-Betreuung sind im Fall der einzelnen Zentren durchwegs unterschiedlich. Der Einsatz für der Inkubation vor und nachgelagerten Aufgaben der AplusB-Zentren ist im Verhältnis zur Inkubationstätigkeit zu bewerten. Es wird daher eine klare Festlegung in den angestrebten Outputs in Abstimmung mit den von den Trägerorganisationen und regionalen Fördereinrichtungen erbrachten Leistungen empfohlen.

- Die betreuten Gründungen sehen einen wesentlichen Mehrwert in der Vernetzungstätigkeit und von den AplusB-Zentren begleiteten Kooperationsanbahnung. Die Zusammenarbeit zwischen Spin-Offs und Industrieunternehmen („Industriekooperationen“) nimmt an Bedeutung zu. Hier fehlt es gegenwärtig an klaren Zielen.

Ähnlich positiv wurde die Möglichkeit zum Austausch zwischen den GründerInnen innerhalb der Zentren bewertet, die unabhängig davon, ob die Gründungsvorhaben im Zentrum beherbergt werden, stattfinden können. Es wird angeregt, ähnliche Möglichkeiten auch auf der nationalen Ebene zu ermöglichen und pflegen.

- Der wachsenden Bedeutung von Geschlechtervielfalt und Diversität, und damit auch eine Erhöhung des Potentials von Frauen als potentielle GründerInnen, ist durch Verbesserung bei gendergerechten Angebote, wie es sie in einigen Zentren bereits existieren, Rechnung zu tragen.

- In Hinblick auf das Berichtswesen und laufende Monitoring werden folgende Weiterentwicklungen angeregt: Wo dies sinnvoll ist, sollte die Dokumentation in der Form eines Monitoringsystems durchgeführt werden, das mit den gesetzten Zielen korrespondierende, nachvollziehbare Indikatoren umfasst. Dies betrifft eine konsistente Abbildung der Outputs (bspw: TeilnehmerInnen bei Veranstaltungen des AplusB-Zentrums) oder auch relevanter Meilensteine in der Entwicklung der Projekte. Des Weiteren wird eine Weiterentwicklung des Gründungs-Monitorings empfohlen. Das Monitoring differenziert nicht ausreichend zwischen den antwortenden GründerInnen und den erfassten Einheiten des Gründungsvorhabens (in weiterer Folge: Unternehmen). Des Weiteren ist es in der Beobachtung der betreuten Fälle nach der Gründung sehr eingeschränkt. 


\section{Anhang}

\subsection{Interviewliste}

\begin{tabular}{|c|c|c|}
\hline Nr. & Name & Zentrum, Einrichtung, Unternehmen \\
\hline \multicolumn{3}{|c|}{ ZentrumsleiterInnen } \\
\hline 1 & Becke, Florian & CAST, Tirol \\
\hline 2 & Buchberger, Bernd & Zentrum für angewandte Technologie, Leoben Steiermark \\
\hline 3 & Fialka, Irene & INiTS, Wien \\
\hline 4 & Ibovnik, Karin & build!, Kärnten \\
\hline 5 & Manz, Markus & Tech2B, Oberösterreich \\
\hline 6 & Moll, Michael & Accent, Niederösterreich \\
\hline 7 & $\begin{array}{l}\text { Steinkogler-Scherzer, } \\
\text { Gabriele }\end{array}$ & Business Creation Center (BCCS), Salzburg \\
\hline 8 & Wutschek, Emmerich & Science Park Graz, Steiermark \\
\hline \multicolumn{3}{|c|}{ Programmleitung und -administration, Kooperations- und Netzwerkpartner } \\
\hline 1 & Chladek, Maria & bmvit, Programmleitung AplusB \\
\hline 2 & Delapina, Franz & NFB \\
\hline 3 & Ebersberger, Bernd & $\mathrm{MCI}$ \\
\hline 4 & Faulhammer, Friedrich & Donau Uni Krems \\
\hline 5 & Freidl, Michael & Universität Graz, Forschungsmanagement und -service \\
\hline 6 & Glößl, Josef & $\begin{array}{l}\text { Universität für Bodenkultur Wien, Vizerektor für Forschung und } \\
\text { Internationale Forschungskooperation }\end{array}$ \\
\hline 7 & Hamm-Riedler, Alexandra & Universität Innsbruck, Beteiligungsgesellschaft \\
\hline 8 & Karg, Peter & Technische Universität Wien, Transferstelle \\
\hline 9 & Kelly Spillmann, Ingrid & Universität Wien, Transferstelle \\
\hline 10 & Laimgruber, Silvia & FFG, Programmmanagement AplusB \\
\hline 11 & Löschnauer, Jürgen & Montanuniversität Leoben \\
\hline 12 & Matt-Leubner, Sara & Universität Innsbruck, Beteiligungen und Industriekooperationen \\
\hline 13 & Müller, Irmgard & WKOÖ \\
\hline 14 & Oliva, Martin & FREQUENTIS startup Center \\
\hline 15 & Pfeiffer, Karl-Peter & FH Joanneum \\
\hline 16 & Ruckenbauer, Christine & Veterinärmedizinische Universität Wien, Transferstelle \\
\hline 17 & Sammer, Werner & Ideentriebwerk Graz \\
\hline
\end{tabular}




\begin{tabular}{|l|l|l|}
\hline 18 & Reisinger, Matthias & Impact Hub Vienna \\
\hline 19 & Riedler, Peter & Universität Graz \\
\hline 20 & Schwaha, Karl & Universität Wien \\
\hline 21 & Steiner, Karin & FH Kufstein, Studiengangsleitung Unternehmensführung \\
\hline 22 & Stelzer, Christopher & Kubator Gmünd \\
\hline 23 & Sumnitsch, Alexander & Wirtschaftskammer Steiermark, Leoben \\
\hline 24 & Schwarz, Erich & AAU \\
\hline 25 & Tangemann, Kirsten & Quadris Consulting, AplusB-Plattform \\
\hline Ehemalige und aktuell betreute Gründungsprojekte \\
\hline 1 & Holzer, Georg & Xamoom GmbH, Klagenfurt \\
\hline 2 & Kaar, Christian & Runtastic (Fragebeantwortung per Email) \\
\hline 3 & Leonhartsberger, Martin & Cumulo, Linz \\
\hline 4 & Moser, Michael & Eologix Sensor Technology, Graz \\
\hline 5 & Primus, Thomas & Foodnotify, Wien \\
\hline 6 & Struska, Nino & Hydroconnect, Ybbsitz \\
\hline 7 & Schimper, Christian & Acticell, Wien \\
\hline & &
\end{tabular}

\subsubsection{TeilnehmerInnen Fokusgruppen}

\section{Nr. Name \\ Zentrum, Einrichtung, Unternehmen}

Fokusgruppe Zentrumsmanagement

\begin{tabular}{|l|l|l|}
\hline 1 & Becke, Florian & CAST, Tirol \\
\hline 2 & Fialka, Irene & INiTS, Wien \\
\hline 3 & Ibovnik, Karin & build!, Kärnten \\
\hline 4 & Manz, Markus & Tech2B, Oberösterreich \\
\hline 5 & Moll, Michael & Accent, Niederösterreich \\
\hline 6 & Weber, Bernhard & Science Park Graz, Steiermark \\
\hline
\end{tabular}

Fokusgruppe Trägereinrichtungen

\begin{tabular}{|l|l|l|}
\hline 1 & Agneter, Doris & tecnet Equity \\
\hline 2 & Auer, Carolin & Medizinische Univ. Graz \\
\hline 3 & Bauer, Ulrich & Techn. Univ. Graz \\
\hline 4 & Ehrentraut, Kurt & FH OÖ \\
\hline 5 & Gohm, Harald & Standortagentur Tirol \\
\hline 6 & Habitzel, Kurt & Universität Innsbruck \\
\hline
\end{tabular}




\begin{tabular}{|l|l|l|}
\hline 7 & Josten, Peter & Medizinische Universität Innsbruck \\
\hline 8 & Mühlburger, Martha & Montan-Universität Leoben \\
\hline 9 & Pamminger, Werner & Business Upper Austria \\
\hline 10 & Patzelt, Petra & RIZ \\
\hline 11 & Resch, Wolfgang & Johannes - Kepler Universität \\
\hline 12 & Rhomberg, Elisabeth & MCI Management Center Innsbruck \\
\hline 13 & Riedler, Peter & Universität Graz \\
\hline 14 & Schönegger, Hans & BABEG \\
\hline 15 & Seidler, Sabine & Technische Universität Wien \\
\hline 16 & Tatzberger, Gabriele & Wirtschaftsagentur Wien \\
\hline 17 & Werner, Joachim & FH Kärnten \\
\hline Fokusgruppe Förderungsstellen & \\
\hline 1 & Czernohorszky, Eva & Wirtschaftsagentur Wien \\
\hline 2 & Ebner, Martina & WST3 \\
\hline 3 & Hammermüller, Christian & Land OÖ \\
\hline 4 & Kink, Manfred & SFG \\
\hline 5 & Kopeinig, Jürgen & KWF - Kärntner Wirtschaftsförderungsfonds \\
\hline 6 & Lorber, Reinhard & Stadtamtsdirektion Leoben \\
\hline 7 & Morianz, Andreas & Stadt Graz, Wirtschaftsabteilung \\
\hline 8 & Priedl, Irma & WST3 \\
\hline 9 & Seyrling, Rainer & Land Tirol, Abt. Wirtschaft u Arbeit \\
\hline 10 & Stellnberger, Robert & KommerzCenter Hochsteiermark Sparkasse \\
\hline 11 & Walch, Franz & NÖBEG \\
\hline
\end{tabular}

\subsection{Fallstudien}

\begin{tabular}{|l|l|}
\hline Unternehmen & Gründungsjahr \\
\hline coccinella-media/ sticklett & 2014 \\
\hline MTA-Systems & 2010 \\
\hline AFreeze GmbH & 2008 \\
\hline Marinomed Biotechnologie GmbH & 2006 \\
\hline PRSG - Pharmaceutical and Regulatory Services GmbH & 2012 \\
\hline NaKu - Natürlicher Kunststoff & 2007 \\
\hline LOGMEDIA & 2008 \\
\hline
\end{tabular}




\subsubsection{Sticklett: „Die Idee muss verständlich sein.“}

\section{Eckdaten zum Unternehmen}

\begin{tabular}{|l|l|}
\hline Unternehmen: & coccinella-media/sticklett \\
\hline $\begin{array}{l}\text { AplusB-Services } \\
\text { genutzt ja/nein }\end{array}$ & Ja, Business Plan Wettbewerb, Unternehmen nutzt Infrastruktur des build! \\
\hline Gründungsjahr: & 2014 \\
\hline Standort: & Viktring, Kärnten / Lakesidepark Klagenfurt \\
\hline Gründerin: & DI Michaela Schicho \\
\hline Firmenstruktur: & Einzelunternehmen \\
\hline Anzahl Mitarbeitende: & -- \\
\hline $\begin{array}{l}\text { Branche, } \\
\text { Produkte/Services }\end{array}$ & $\begin{array}{l}\text { Bekleidung/Wearable Technologies/Smart Clothing: dekorative Applikationen } \\
\text { zur Erfassung von Vitalfunktionen auf der Kleidung (Label: sticklett/coccinella } \\
\text { Medica -für fürsorgliche Applikation) }\end{array}$ \\
\hline
\end{tabular}

\subsubsection{MTA-Systems: „Am Anfang weiß man gar nicht, was man alles nicht weiß ...“}

\section{Eckdaten zum Unternehmen}

\begin{tabular}{|l|l|}
\hline Unternehmen: & MTA-Systems \\
\hline $\begin{array}{l}\text { AplusB-Services } \\
\text { genutzt ja/nein }\end{array}$ & Ja, tech2b (extern in Gründungsprogramm - gate-stage, B2E) \\
\hline Gründungsjahr: & 2010 \\
\hline Standort: & Perg /OÖ \\
\hline GründerInnen: & Gliewe Mario, Ing. Feuerstein Herwig, DI Pankraz Dietmar \\
\hline Firmenstruktur: & $\begin{array}{l}\text { GmbH; 6 Einzelpersonen als Gesellschafter: Herwig Feuerstein (25\%), Mario } \\
\text { Gliewe (25\%), Dietmar Pankraz (25\%), Christiane Feuerstein-Brandstetter } \\
(8,33 \%), \text { Renate Gliewe (8,33\%), Andrea Pankraz (8,33\%) }\end{array}$ \\
\hline Anzahl Mitarbeitende: & 12 Personen (9 Vollzeit/3 Teilzeit) \\
\hline $\begin{array}{l}\text { Branche, } \\
\text { Produkte/Services: }\end{array}$ & „Individuallösungen“ in der Antriebs- und Automatisierungstechnik \\
\hline
\end{tabular}




\subsubsection{AFreeze: „Gründung ist ein Weg, um einen Jugendtraum zu erfüllen“}

Eckdaten zum Unternehmen:

\begin{tabular}{|l|l|}
\hline Unternehmen: & AFreeze GmbH \\
\hline $\begin{array}{l}\text { AplusB-Services } \\
\text { genutzt ja/nein }\end{array}$ & Ja (CAST) \\
\hline Gründungsjahr: & 2008 \\
\hline Standort: & Innsbruck \\
\hline GründerInnen: & Dr. Gerald Fischer, Dr. Florian Hintringer \\
\hline Firmenstruktur & GmbH \\
\hline Anzahl Mitarbeitende & 15 \\
\hline $\begin{array}{l}\text { Branche, } \\
\text { Produkte/Services }\end{array}$ & $\begin{array}{l}\text { Medizintechnik: Produkte zur Behandlung von PatientInnen mit Herzrhythmus- } \\
\text { störungen; CoolLoop: Kryoablationskatheter }\end{array}$ \\
\hline
\end{tabular}

\subsubsection{Marinomed: „Erfolgsrezept: innovativ, unorthodox, kreativ, pragmatisch“}

\section{Eckdaten zum Unternehmen}

\begin{tabular}{|l|l|}
\hline Unternehmen: & Marinomed Biotechnologie GmbH \\
\hline $\begin{array}{l}\text { AplusB-Services } \\
\text { genutzt ja/nein }\end{array}$ & Ja (INiTS) \\
\hline Gründungsjahr: & 2006 \\
\hline GründerInnen: & $\begin{array}{l}\text { Andreas Grassauer (CEO) } \\
\text { Eva Prieschl-Grassauer (CSO) }\end{array}$ \\
\hline Firmenstruktur & $\begin{array}{l}\text { GmbH } \\
6 \text { Gesellschafter: Hr. u Fr Grassauer, (je ca. 15\%) } \\
\text { Thomas Friedrich (ca. 10\%), VETWIDI Forschungsholding GmbH (4,5\%), } \\
\text { Hermann Unger (15,6\%), Acropora Beteiligungs GmbH (ca. 38,26\% - Haupt- } \\
\text { eigner) }\end{array}$ \\
\hline Anzahl Mitarbeitende & 24 \\
\hline $\begin{array}{l}\text { Branche, } \\
\text { Produkte/Services }\end{array}$ & Biopharmazie: Entwicklung von frei erhältlichen antiviralen Pharmazeutika \\
\hline
\end{tabular}




\subsubsection{Pharmaceutical and Regulatory Services GmbH: „eine Gründung ist nicht für jede/n empfehlenswert“"}

\section{Eckdaten zum Unternehmen}

\begin{tabular}{|l|l|}
\hline Unternehmen: & PRSG - Pharmaceutical and Regulatory Services GmbH \\
\hline $\begin{array}{l}\text { AplusB-Services } \\
\text { genutzt ja/nein }\end{array}$ & nein \\
\hline Gründungsjahr: & 2012 \\
\hline Standort: & Graz \\
\hline GründerInnen: & Team \\
\hline Firmenstruktur: & $\begin{array}{l}\text { GmbH, 3 Gesellschafter: Lydia Langkammer (46\%), Ernest Schinewitz (44\%), } \\
\text { Annemarie Moretti (10\%) }\end{array}$ \\
\hline $\begin{array}{l}\text { Anzahl Mitarbeitende } \\
\text { Branche, } \\
\text { Produkte/Services: }\end{array}$ & $\begin{array}{l}\text { Pharmazie } \\
\text { Beratung/DL in Arzneimittelzulassung }\end{array}$ \\
\hline
\end{tabular}

\subsubsection{NaKu - Aus Natürlichem Kunststoff: „Von Technik bis zum fertigen Produkt ist es ein langer Weg“"}

Eckdaten zum Unternehmen

\begin{tabular}{|l|l|}
\hline Unternehmen & $\mathrm{NaKu}-$ Natürlicher Kunststoff \\
\hline $\begin{array}{l}\text { AplusB-Services } \\
\text { genutzt ja/nein }\end{array}$ & Nein \\
\hline Gründungsjahr & 2007 \\
\hline Standort & Wiener Neustadt \\
\hline GründerInnen & Ute und Johann Zimmermann \\
\hline Firmenstruktur: & Einzelunternehmen \\
\hline Anzahl Mitarbeitende & 5 \\
\hline $\begin{array}{l}\text { Branche, } \\
\text { Produkte/Services }\end{array}$ & Produktion und Verkauf unterschiedlicher Natürliche Kunststoffe \\
\hline
\end{tabular}




\subsubsection{LOGMEDIA: „Wachstum ist eine Grundsatzentscheidung“}

\section{Eckdaten zum Unternehmen}

\begin{tabular}{|l|l|}
\hline Unternehmen: & LOGMEDIA \\
\hline $\begin{array}{l}\text { AplusB-Services } \\
\text { genutzt ja/nein }\end{array}$ & nein \\
\hline Gründungsjahr: & 2008 \\
\hline Gründer: & Ing. Marc Gfrerer, MBA \\
\hline Firmenstruktur: & GmbH, 1 Gesellschafter \\
\hline Anzahl Mitarbeitende: & 10 \\
\hline $\begin{array}{l}\text { Branche, } \\
\text { Produkte/Services }\end{array}$ & IT, Werbung (Programmierung und Web-Entwicklung) \\
\hline
\end{tabular}


JOANNEUM RESEARCH

Forschungsgesellschaft mbH

Leonhardstraße 59

8010 Graz

Tel. +43 316 876-0

$\mathrm{Fax}+43316$ 876-1181

pr@joanneum.at

www.joanneum.at 